Book Chapter published; to be referenced as:

Maragos, P. (2013). "Representations for Morphological Image Operators and Analogies with Linear Operators". In Advances in Imaging and Electron Physics, vol. 177 (edited by P.W. Hawkes), pp. 45-187. Academic Press: Elsevier Inc.

\title{
REPRESENTATIONS FOR MORPHOLOGICAL IMAGE OPERATORS AND ANALOGIES WITH LINEAR OPERATORS
}

\author{
Petros Maragos \\ National Technical University of Athens \\ School of Electrical \& Computer Engineering \\ Zografou 15773, Athens, Greece. \\ Email: maragos@cs.ntua.gr
}

\section{Contents}

1 Introduction $\quad 3$

1.1 Why a representation theory? . . . . . . . . . . . . . . . . 3

1.2 Overview of Developments in Morphological Operators and Related Areas . . . . . . 5

1.3 Summary of Representation Theory Topics covered . . . . . . . . . . . . . . . . . 9 9

1.4 Notation . . . . . . . . . . . . . . . . . . . . . . . . . . . . . . 11

2 Linear Spaces and Linear Image Operators $\quad 11$

2.1 Linear Spaces . . . . . . . . . . . . . . . . . . . . . . . . . . . 11

$2.1 .1 \quad$ Algebraic Structure _. . . . . . . . . . . . . . . . . . . 12

2.1.2 Topological Structure, Banach spaces . . . . . . . . . . . . . . . . . 13

2.1.3 Geometric Structure, Hilbert spaces . . . . . . . . . . . . . . . . . . 13

2.1.4 Examples of Linear Image Spaces . . . . . . . . . . . . . . . . . . . . . . . . 14

2.2 Linear Operators . . . . . . . . . . . . . . . . . . . . . . 16

2.2.1 Algebraic Definitions for Linear Operators . . . . . . . . . . . . . . 16

2.2.2 Linear Operators on Normed Spaces . . . . . . . . . . . . . . . 17

2.2.3 Linear Operators on Inner Product Spaces . . . . . . . . . . . . . . . . 17

2.2 .4 Isomorphisms . . . . . . . . . . . . . . . . . . . . . . . . 18

2.3 Linear Projections . . . . . . . . . . . . . . . . . . . . . . . . 19

2.3.1 Algebraic Decomposition . . . . . . . . . . . . . . . . . . 19

2.3.2 Orthogonal Projections . . . . . . . . . . . . . . . . . . . . . 19

2.4 Representations of Linear Operators . . . . . . . . . . . . . . . . . . . . . 20

2.4.1 Matrix Representation of Linear Operators on Finite-dimensional Vector Spaces 20

2.4.2 Riesz Representation Theorem and Linear Convolution . . . . . . . . . . . . 21

2.4.3 Spectral Representation of Linear Operators on Finite-dimensional Vector

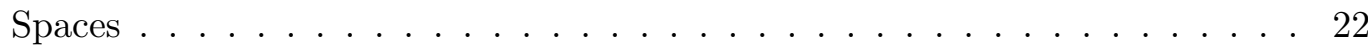

3 Lattice Spaces and Morphological Image Operators 23

3.1 Lattices: Synopsis . . . . . . . . . . . . . . . . . . . . . . . 23

3.1 Lattice Properties . . . . . . . . . . . . . . . . . . . . 24

3.1 .2 Semilattices . . . . . . . . . . . . . . . . . . . . 24

3.1.3 Sublattices and Sup/Inf-closed subsets . . . . . . . . . . . . . . 25

3.1 .4 Sup-generators, Atoms . . . . . . . . . . . . . . . . . . . . 26 
3.1.5 Boolean Lattices . . . . . . . . . . . . . . . . . . . . 26

3.1 .6 Function Lattices . . . . . . . . . . . . . . . . . . . 27

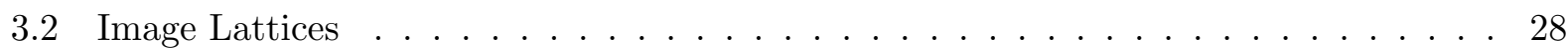

3.3 Image and Signal Operators on Lattices . . . . . . . . . . . . . . . . . 29

3.4 Monotone Lattice Operators . . . . . . . . . . . . . . . . . . . . 30

3.4 Increasing Operators . . . . . . . . . . . . . . . . 30

3.4.2 Decreasing Operators . . . . . . . . . . . . . . . 31

3.5 Adjunctions, Dilations, Erosions _ . . . . . . . . . . . . . . 32

3.6 Shift-varying Dilation and Erosion . . . . . . . . . . . . . . . 34

3.6.1 Structuring Element Map $(\mathrm{SEM}) \ldots \ldots \ldots \ldots \ldots \ldots$

3.6 .2 Adaptive Morphology . . . . . . . . . . . . . . . . . . 36

3.7 Convergence and Continuity on Lattices . . . . . . . . . . . . . . . . 36

3.7.1 Convergence and Continuity on Topological Image Spaces . . . . . . . . . . . 36

3.7.2 Order Convergence and Continuity on Lattices . . . . . . . . . . . . . . . . 37

3.8 Openings, Closings, Order Projections . . . . . . . . . . . . . . 38

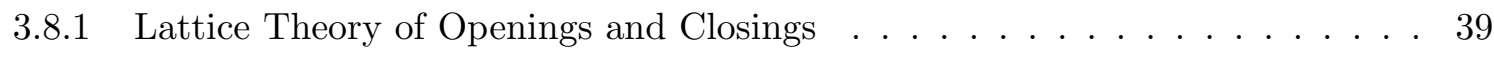

3.8.2 Examples of Openings and Closings . . . . . . . . . . . . . . . 40

3.8.3 Examples of Order Projections . . . . . . . . . . . . . . . . . . . 44

4 Minimax Algebra and Image Operators on Complete Weighted Lattices 44

4.1 Lattice-Ordered Monoids . . . . . . . . . . . . . . . . . . . . . 45

4.2 Clodum: An Algebraic Structure for Weighted Lattice Arithmetic . . . . . . . . . . . 46

4.3 Nonlinear Spaces based on Clodums: Complete Weighted Lattices . . . . . . . . . . 47

4.3 .1 General Algebraic Structure . . . . . . . . . . . . . . . . . 47

4.3.2 Sup/Inf Span, Independence, Basis, Dimension . . . . . . . . . . . . . . . 49

4.3 .3 Complete Weighted Lattices of Functions . . . . . . . . . . . . . . . . . 49

4.4 Image Operators on Complete Weighted Lattices . . . . . . . . . . . . . . . . . 50

4.4.1 Image Space, Impulse Representations and Vertical Translations . . . . . . . 50

4.4.2 Representation of Dilations and Erosions Invariant Under Vertical Translations 51

4.5 Representation of Signal Dilation and Erosion Operators Invariant under Translation

Monoids on Complete Weighted Lattices . . . . . . . . . . . . . . . . 53

4.5.1 Generalized Translations . . . . . . . . . . . . . . . . . . 53

4.5.2 Generalized Convolution Representation of $\mathbb{T}$-Invariant Dilations and Erosions 55

4.5.3 Generalized Convolution Adjunctions . . . . . . . . . . . . . . 56

4.6 Special Cases . . . . . . . . . . . . . . . . . . . . . 57

4.6 .1 Max-Plus Image Operators . . . . . . . . . . . . . . . 57

4.6 .2 Max-Product Image Operators ． . . . . . . . . . . . . . . . . 58

4.6.3 Weighted Lattice Operators using Fuzzy Norms . . . . . . . . . . . . . . . . . 59

4.7 Matrix Representations of Image Operators on Finite-dimensional Weighted Mini$\max$ Vector Spaces . . . . . . . . . . . . . . . . . 6 . 63

4.8 Elements from Max-plus Matrix Algebra and Spectral Analysis . . . . . . . . . . 66

5 Kernel and Basis Representations of Operators on Lattices $\quad 68$

5.1 Kernel Representation of TI Increasing Set Operators . . . . . . . . . . . . . . 68

5.2 Basis Representation of TI Increasing Set Operators . . . . . . . . . . . . . . . . . . 69

5.3 Examples of Set Operator Bases _. . . . . . . . . . . . . . . 71

5.3 .1 Morphological Set Operators . . . . . . . . . . . . . . . 71

5.3 .2 Median and Rank Filters for Sets . . . . . . . . . . . . . . . . . . 72

5.3.3 Window Transformations for Shape Detection and Locally-defined Set Oper-

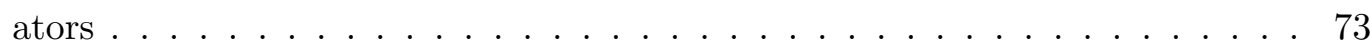

5.4 Kernel and Basis Representations for TI Increasing Function Operators . . . . . . . 75

5.4.1 Representation of Weighted Operators and Basis Approximations . . . . . . . 75 
5.4 .2 Representation of Flat Operators . . . . . . . . . . . . . . . . . . 77

5.4.3 Representations of Boolean Functions and Stack Filters . . . . . . . . . . . 78

5.4.4 Representation of Linear Operators via Morphological Operations . . . . . . 80

5.5 Representations for Spatially-Varying Increasing Operators . . . . . . . . . . . . . 82

5.6 Representations for TI Non-Increasing Operators . . . . . . . . . . . . . . . . 85

5.7 Representations for TI Increasing Operators on Complete Weighted Lattices . . . . . 87

5.8 Representations for TI Openings and Closings . . . . . . . . . . . . . . . . 89

6 Conclusions

\begin{abstract}
This chapter deals with representation theoretical issues of nonlinear image operators, mainly based on the methodology of mathematical morphology, and more generally operators on lattices. After a brief overview of developments in morphological image operators both chronologically and thematically, the chapter provides a survey of some main concepts and results in the theory of lattices and morphological operators, especially of the monotone type. It also provides comparisons with linear operator theory. Then, it introduces a nonlinear signal space called complete weighted lattice, which generalizes both mathematical morphology and minimax algebra. Afterwards, it focuses on the representation of translation-invariant and/or increasing operators either on Euclidean spaces (or their discretized versions) or on complete weighted lattices by using a nonlinear basis. The results are operator representations as a supremum or infimum of nonlinear convolutions that are either of the max-plus type or their generalizations in weighted lattices. These representations have several potential applications in computation, imaging and vision, and nonlinear functional analysis.
\end{abstract}

Keywords: image operators, representation, nonlinear basis, supremal convolution, mathematical morphology, lattices, minimax algebra.

\title{
1 Introduction
}

\subsection{Why a representation theory?}

In general, we believe that, it helps conceptually and offers insight to know that a particular system together with many others sharing a few common properties result from the combination of a few simple generic systems.

Image representations are important both for computational processing of image data, intermediate transformations and feature extraction as well as for higher-level cognitive tasks such as building symbolic descriptions and ultimate image understanding (Marr, 1982). Nowadays, in the digital era, the problems with analyzing and managing the information in big data, much of which is visual, make the issue of more efficient image representations even more acute, where aspects of efficiency may include compactness, sparseness, and integration of low-level (numeric) with high-level (semantic) processing.

This chapter advances the thesis that, with the current availability in image/signal processing and in computer vision of a large variety of image operator ${ }^{1}$ types, e.g. linear versus nonlinear, continuous versus discrete, local versus global, single-scale or multi-scale, functional versus graphical, the need also arises to study the representation of operators. The field of linear operator theory is already well studied, e.g. the field of linear vector spaces and corresponding functional analysis. In this chapter we focus on representation theoretical issues of nonlinear image operators, mainly of the morphological type, i.e. based on the methodology of mathematical morphology, and more generally operators on lattices. From the viewpoint of serving as inputs to and outputs from the operators, images will be represented as elements of collections of multidimensional sets or signals; thus, by 'image operators' we shall also include the study of arbitrary signal operators but the

\footnotetext{
${ }^{1}$ Throughout this chapter, by 'operator' we shall mean a mapping, transformation, or general system processing images or general multidimensional signals of finite or infinite extent.
} 
emphasis will be on images. We also provide comparisons with linear operator theory, both to contrast differences and/or similarities but also to often draw inspiration for new developments in morphological and lattice operators.

In algebra, 'representation theory' usually means a branch of mathematics where some abstract algebraic structures are described using concepts of linear algebra; for example, elements of a group are represented as linear operators on vector spaces and the group binary operation (addition or multiplication) is represented as a matrix addition or multiplication. Representations of systems with group of matrices is also important in physics; e.g. symmetry groups allowed the discovery of new particles. In this chapter we broaden the term 'representation' and by 'representation of operators' we shall mean their equivalent expression or realization as a combination of simpler components. This combination may be serial, e.g. a composition, or parallel, i.e. a superposition of simpler operations. For example the spectral representation of linear operators in terms of projections, or (as explained in this chapter) the representation of broad classes of nonlinear operators in terms of elementary operators that are called dilations and erosions and play the role of building blocks for more complex systems. Sometimes, by representation we may also mean the alternative expression of the original operator based on some other well-known or more easily understood operations. Examples include the representation of a linear shift-invariant operator as a linear convolution, or the representation of a linear operator on finite-dimensional spaces as the multiplication of input signal vectors by a matrix, or (as explained in this chapter) the representation of some nonlinear operators as nonlinear convolutions of the max-plus type or in the finite-dimensional case as nonlinear matrix operations.

As we shall summarize later in this introduction, morphological operators are not used only in image processing and computer vision (Matheron, 1975; Serra, 1982; Maragos and Schafer, 1990; Heijmans, 1994; Haralick and Shapiro, 1992; Maragos, 1998), but also in several diverse areas such as neural nets (Davidson and Hummer, 1993; Yang and Maragos, 1995; Pessoa and Maragos, 2000; Ritter et al., 1998; Ritter and Urcid, 2003), convex analysis and optimization (Rockafellar, 1970; Lucet, 2010), mimimax algebra and its applications in scheduling, networks, and discrete events dynamical systems (Cuninghame-Green, 1979; Cohen et al., 1989). Some of their attractive aspects include their efficiency and their capability of parallel implementations of a large class of systems using simple local operations that do not involve multiplications.

In the area of image computing, there have been specialized platforms whose main operations are combinations of erosions and dilations. Early examples include cellular automata, parallel architectures, VLSI, optical/electronic, and analog optical implementations of morphological and rank filters whose special cases are the simple erosions and dilations (Preston et al., 1979; Sternberg, 1980; Harber et al., 1985; Ochoa et al., 1987; O'Neil and Rhodes, 1986; Hereford and Rhodes, 1988; Barrera et al., 1994). Nowadays, the vast majority of many computer (hardware or software) systems for digital image processing include among their main operations the basic morphological operators such as erosions, dilations, openings and closings.

Therefore, from an computational theory viewpoint, a representation theory for morphological and related lattice image operators establishes the capabilities and limitations of all these computer architectures and implementations by finding the general class of signal operations that they can perform. Three important issues are the following. Analysis: how to decompose existing complex operators into simpler ones? Synthesis: what is the broadest class of high-level image computing systems we can build from simple elementary operations. Language: if we use a formal language whose words for processing binary (graylevel) images are morphological erosions and dilations and the associated lattice operations of union (supremum), intersection (infimum) and complementation (negation), then this language is complete for set operators and expressive since most useful operators can be described only with a few words (Barrera and Banon, 1992). Such issues have also been used for the automatic programming of learning machines for binary image processing and pattern recognition (Barrera and Salas, 1996; Barrera et al., 1997). But how expressive is the language of morphological operators and their lattice generalizations for graylevel image processing? This is one of the major themes addressed in this chapter. 
Further, from an applications viewpoint, the ever-increasing industrial need in automated visual systems (e.g. mobile digital cameras or visual sensors) calls for low-cost machine vision modules that can do a variety of complex image processing tasks based on a rather small set of available simple image operations. Hence, given the wide and interdisciplinary applicability of the morphological operators, their parallellism, and their simple implementations, the representations in this chapter theoretically support a computational module that can perform a minimal set of elementary morphological operations, which can synthesize a large variety of more complex systems.

From a nonlinear functional analysis viewpoint, this chapter unifies the representation of previously totally unrelated operators, e.g., morphological filters used in image analysis, median (or other order-statistic based) filters used in robust statistics, shape detection transformations, and even digital linear FIR filters used in signal processing. It also provides a common mathematical framework and analytic tools for a large class of linear and nonlinear operators; this may help the analysis and design of hybrid linear/nonlinear systems, as for example in (Pessoa and Maragos, 1998). Another goal of this chapter is to shed more light into some analogies between morphological versus linear operators. Figure 1 shows an original graylevel image, its linear filtering via a Gaussian convolution and two types of morphological filterings. The linear filter obeys the well-known linear superposition. Are there conceptually similar superpositions obeyed by some morphological operators? The linear shift-invariant filter is represented as a linear convolution with its impulse response, which is a 2D Gaussian function in this example. Are there such concepts and representations for morphological filters? The linear filter has an approximate lowpass action, i.e. it attenuates high frequencies. If it were an ideal-cutoff filter with unity gain, its subsequent iterations would give the same result as its first pass on the input image. We call such an ideal linear filter a projection, i.e. a linear idempotent operator. Are there such operators in morphological filters? Actually, Figs. 1(c),(d) show two morphological projections, i.e. increasing and idempotent filters. The above are some of the theoretical issues addressed in this chapter.

\subsection{Overview of Developments in Morphological Operators and Related Areas}

Before we outline our scope of coverage on morphological operator representation theory, we provide a brief historic tour of developments in the corresponding field of morphological image analysis. Classic mathematical morphology, as a field of nonlinear geometric image analysis, was developed initially by Matheron (1975), Serra (1982) and their collaborators and was applied successfully to geological and biomedical problems of image analysis. In this first period, i.e. the late 1960's and throughout the 1970's, the basic morphological operators were developed first for binary images based on set theory (Matheron, 1975; Serra, 1982) inspired by the work of Minkowski (1903) and Hadwiger (1957), second for graylevel images based on local min/max operators and level sets (Meyer, 1978; Serra, 1982) or on fuzzy sets (Nakagawa and Rosenfeld, 1978; Goetcherian, 1980), and third for graylevel images but with weighted $\mathrm{min} / \max$ operators using a geometric interpretation based on the umbra approach of Sternberg $(1980,1986)$ which is algebraically equivalent to maxplus convolutions. All these operators were translation-invariant (TI) and their set generators were Minkowski set addition and subtraction; thus, we shall refer to them either as Minkowski operators or as Euclidean morphological operators since their most common domain is the Euclidean plane $\left(\mathbb{R}^{2}\right)$ or its discretized version $\left(\mathbb{Z}^{2}\right)$ and they commute with Euclidean translations.

In the 1980s, extensions of classic mathematical morphology and connections to other fields were developed by several research groups worldwide along various directions including: applications to pattern recognition and computer vision problems; unified analysis and representation of large classes of nonlinear filters, including morphological, rank and stack filters (Maragos and Schafer, 1987a,b); multiscale image processing and shape and texture analysis; statistical analysis and optimal design of morphological filters. Accounts and references at varying degrees of detail can be found in books by (Serra, 1982, 1988; Heijmans, 1994; Haralick and Shapiro, 1992; Dougherty and Astola, 1994) or tutorial chapters and papers by (Sternberg, 1986; Haralick et al., 1987; Maragos and Schafer, 1990; Serra and Vincent, 1992; Goutsias, 1992; Maragos, 1998, 2005a) that deal with 


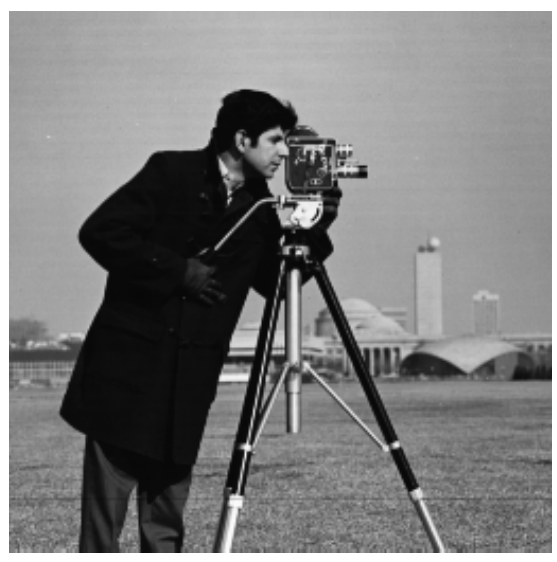

(a)

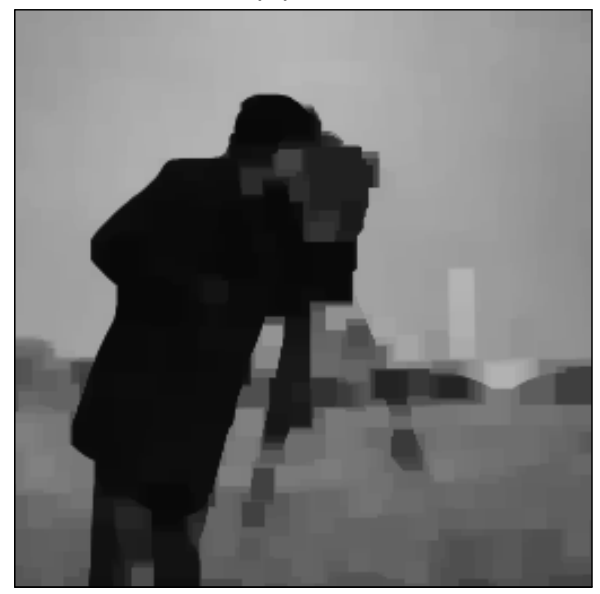

(c)

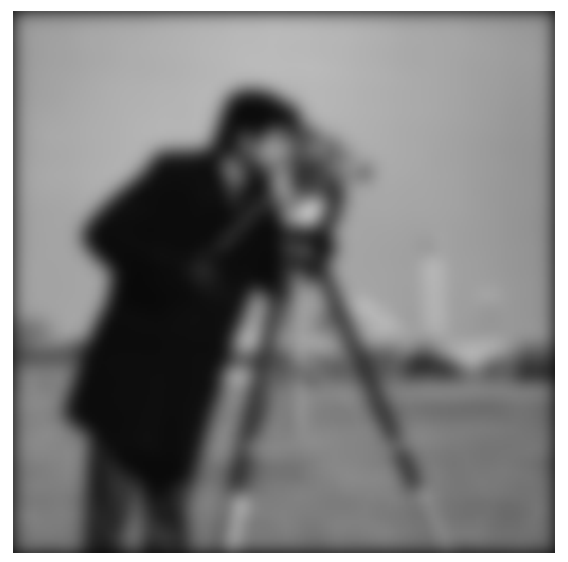

(b)

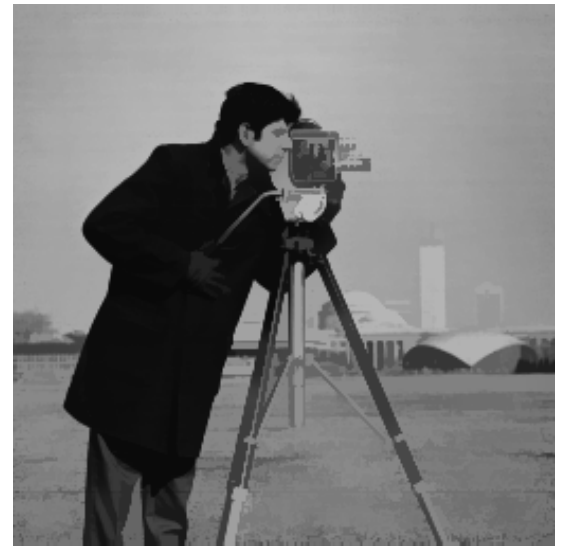

(d)

Figure 1: Linear and morphological image simplification. (a) Original image $f(256 \times 256$ pixels). (b) Linear convolution of $f$ with a Gaussian. (c) Morphological clos-opening of $f$ by a discrete disk-like structuring element. (d) Morphological reconstruction (of the leveling type) of $f$ with respect to a marker which was the Gaussian convolution in (b). Both the linear and the morphological filterings were at the same scale. (The 'scale' parameter is defined as the variance of the Gaussian for the linear convolution, as the radius of the structuring element for the clos-opening and as the scale of the marker for the reconstruction filtering.)

mathematical morphology. Overall, during the first two decades (late 1960's until late 1980's), this whole methodology was essentially a Euclidean morphology where the basic operators could be understood geometrically as translation-invariant set operations based on Minkowski-type set operations and implemented algebraically as nonlinear signal operations, i.e. Boolean or $\mathrm{min} / \mathrm{max}$ superpositions and max-plus convolutions. Its image analysis applications were mainly in denoising, nonlinear multiscale filtering, feature extraction, simple object detection, shape and texture analysis, and watershed-based segmentation.

In the late 1980's and early 1990's a new and more general formalization of morphological operators was introduced: Lattice morphology. Specifically, the need to unify its analysis tools for both binary and gray images as well as to use it for more abstract data types such as graphs led researchers in mathematical morphology to extend its theory by generalizing the image space to a complete lattice and viewing all image transformations as lattice operators. The theoretical foundations of morphology on complete lattices were developed by Serra and Matheron, presented as chapters in Serra (1988), and further extended by Heijmans and Ronse (1990); Ronse and Heijmans (1991), Heijmans (1994), and Roerdink (1993). Later another algebraic approach to 
morphology was developed by Keshet (Keshet, 2000) based not on complete lattices but on a related weaker algebraic structure, inf-semilattices. The basic advance of lattice morphology was to develop more general operators that shared with the standard dilation, erosion, opening and closing only a few algebraic properties. One such fundamental algebraic structure is a pair of erosion and dilation operators that form an adjunction. This guarantees the formation of openings and closings via composition of the adjunction constituents. Other new concepts include the groupinvariant operators (Heijmans and Ronse, 1990; Roerdink, 2000); connected operators (Serra and Salembier, 1993; Salembier and Serra, 1995) and connectivity-based segmentation (Serra, 2000), graph morphology (Vincent, 1989; Heijmans et al., 1992); and the slope transform (Dorst and van den Boomgaard, 1994; Maragos, 1994) defined and studied in Maragos (1994, 1995, 1996) and Heijmans and Maragos (1997) from the viewpoint of lattice morphology. Overall, the lattice framework allows us to unify the concepts and analysis of large classes of operators that share a few fundamental properties, independently of whether they are defined for sets (shapes), binary signals (binary images), multilevel signals (graylevel images), or even more abstract image data types such as graphs. The lattice operators have found many applications in important image analysis computer vision tasks, such as segmentation, shape analysis, motion analysis, and object detection. Figure 1 shows two morphological filters both of which can simplify an image without the severe blurring caused by a linear smoothing filter; from these two morphological filters, the reconstruction filter is based on lattice-theoretic concepts.

During the 1990's, in parallel to the development of lattice morphology, another new development was that of differential morphology (Maragos, 1996). This term contains two subareas, both related to nonlinear dynamical systems: (1) The first subarea combined ideas from linear (Gaussian) scale-space analysis in computer vision based on the linear isotropic heat diffusion partial differential equation (PDE) and from multiscale morphology (Maragos, 1989b) to develop nonlinear PDEs that generate continuous-scale morphological filters (mainly Minkowski-type dilation and erosion). The main three independent contributions in morphological PDEs are (Alvarez et al., 1993), Brockett and Maragos (1994) and van den Boomgaard and Smeulders (1994). For overviews, we refer the interested reader to two tutorial chapters of Guichard et al. (2005) and Maragos (2005c). Connections between the morphological PDEs and the slope transform were developed in Dorst and van den Boomgaard (1994) and Heijmans and Maragos (1997). (2) The second subarea deals with $2 \mathrm{D}$ difference equations modeling distance transforms and their analysis using slope transforms. In this chapter we shall not pursue the analysis of these aspects of morphological operators.

The scientific field of convex analysis and optimization (Rockafellar, 1970; Lucet, 2010), was initially unrelated to mathematical morphology, but it has been using extensively some of the main mathematical tools that morphology has also been using such as max-plus convolution and its dual, called supremal and infimal convolution respectively in convex analysis (Bellman and Karush, 1963; Rockafellar, 1970), and the hypograph of a function which is called 'umbra' in morphology. At the end of 1990's the strong connections between convex analysis and lattice morphology were realized and studied in (Heijmans and Maragos, 1997). Examples include (1) the distance transform, which is expressed via infimal convolution in convex analysis and via max-plus difference equations in digital image analysis Borgefors (1986), and (2) the Legendre-Fenchel conjugate (transform) of convex analysis, which is very closely related to the lattice-based slope transform (Maragos, 1995, 1996; Heijmans and Maragos, 1997). Felzenszwalb and Huttenlocher (2004a) used the connection between distance transform and slope transform to develop a fast distance transform that has found application in computer vision problems such as distance computation and optimization in belief networks (Felzenszwalb and Huttenlocher, 2004b). There is a recent detailed review by Lucet (2010) of convex analysis, slope transforms and related optimization where the crossfertilization between these areas and mathematical morphology was explained from many different aspects. Returning to the area of differential morphology (Maragos, 1996, 2001), this refers to the intersection between image processing with max-plus convolutions, differential calculus, maxmin dynamical systems and convex analysis (distance and slope transforms). Another field that combines ideas from mathematical morphology and convex analysis is digital geometry (Kiselman, 
2003). In this chapter we shall not pursue the analysis of these aspects of morphological operators.

In the 1980's an effort started to unify all digital image operations under a common image algebra amenable to computation. The term 'image algebra' was first coined by Sternberg (1980) but it referred only to the algebraic structure of mathematical morphology. Obviously, classic (Minkowski) erosions and dilations by finite structuring elements are insufficient by themselves to represent all possible image operations. In parallel to the development of mathematical morphology, there has been another independent effort in the 1980's by Ritter and his collaborators (Ritter and Gader, 1987; Ritter and Wilson, 1987) to develop a more complete image algebra that represents all digital finite-extent image-to-image operations as a finite composition of a few basic operations, which include Minkowski-type erosions and dilations; a subalgebra of their full image algebra covers the classic part of mathematical morphology. The goal of the image algebra by Ritter and his coworkers was to unify all digital image operations (linear and nonlinear) using traditional algebraic structures ${ }^{2}$ e.g. groups, rings, fields, vector spaces, monoids, semirings. A fusion of image algebra and lattice structures was done in Davidson (1993). The culmination of all these efforts can be found in the book by Ritter and Wilson (2001).

For problems in fields totally separate from image processing, e.g. scheduling and operations research, Cuninghame-Green $(1979,1994)$ has developed a nonlinear matrix algebra called minimax algebra, also known as max-plus algebra, where he has exploited the interaction of the $\max / \mathrm{min}$ idempotent algebraic structure with the group structure of real addition + and has developed analogies with the product-of-sums structure of linear algebra. Minimax algebra was not originally developed for image analysis. Its traditional applications areas were and still are in scheduling (e.g. material flow in automated manufacturing, traffic flow in transportation or communication networks) and operations research (Cuninghame-Green, 1979), shortest path problems in graphs (Peteanu, 1967), as well as in algebraic modeling of discrete event control systems (DEDS) (Cohen et al., 1989). The latter applications area (DEDS) shares some system theoretic aspects with the operator representation issues discussed in this chapter. Minimax algebra is a nonlinear matrixoriented algebra, where the underlying archetypal structure for scalars is the set $\overline{\mathbb{R}}=\mathbb{R} \cup\{-\infty,+\infty\}$ of extended reals equipped with max or min operations and addition. Its basic operators are maxplus or dual min-plus generalized products of matrices with vectors where the standard multiplication of a row vector (from left) with a column vector (from right) is done via a max-of-additions instead of the standard sum of products of linear algebra.

Thus, in addition to mathematical morphology (i.e. its Euclidean and lattice-based versions), we have mentioned so far two other related algebraic systems, image algebra and minimax algebra. All three systems have had some theoretical missing parts for completion. Both the image algebra and the minimax algebra use min-max superpositions, max-plus arithmetic and some concepts from lattice theory. A fusion of concepts from image algebra and minimax algebra was also done by Davidson (1993). However, the above efforts have not exploited the complete lattice structure to the level that mathematical morphology has done and have not focused on the concept of lattice operators and especially adjunctions (Galois connections). Further, both they have remained in the finite-dimensional case. Minimax algebra is a matrix algebra over finite-dimensional vector spaces. Similarly, image algebra (Ritter and Wilson, 2001) deals with finite-extent digital images either by processing them with finite templates in the spatial domain or via finite discrete transforms (e.g. the discrete Fourier transform) in the frequency domain. From both approaches there seems to be missing the case of working over infinite-dimensional spaces; e.g. morphological transformations with infinite-extent structuring functions either on a continuous or a discrete domain. Missing also is the complete lattice structure which allows infinite signal superpositions based on supremum and infimum operations. From the other side, of importance for the weighted lattice operators discussed in this chapter is to note that, both Euclidean and lattice-based mathematical morphology have focused on and exploited mainly the standard lattice structure, i.e. supremum and infimum (often abbreviated as sup/inf) superpositions which become maximum and minimum in the finite case. Although some useful operations in mathematical morphology combine the sup/inf

\footnotetext{
${ }^{2}$ For definitions of the basic algebraic structures, a general reference is Lang (2005).
} 
with max-plus arithmetic (e.g., Minkowski operations with gray structuring elements, chamfer distance transforms), such cases have always remained a minority in mainstream morphological image analysis.

Maragos (2005a) bridged the above gaps and fused lattice-based mathematical morphology with minimax algebra, by allowing for both finite- as well as infinite-dimensional spaces and for sup/inf superpositions over infinite signal collections. Toward this goal, a more general algebraic structure was introduced, called 'clodum' (complete lattice-ordered double monoid), that combines the sup/inf lattice structure with a scalar semi-ring arithmetic that possesses generalized 'additions' and *-'multiplications'. This clodum structure enabled him to develop a unified analysis for: (i) representations of translation-invariant operators compatible with these generalized algebraic structures as nonlinear (sup/inf) convolutions, and (ii) representations of all increasing translation-invariant operators as suprema of such nonlinear convolutions with functions from a special collection that characterizes the operator. Special cases of this unification include generalized Minkowski operators and lattice fuzzy image operators. Applications of this nonlinear signal algebra have appeared in (Maragos and Tzafestas, 1999; Maragos et al., 2000) for max-plus nonlinear control and in (Maragos et al., 2001, 2003) for image analysis based on fuzzy logic.

\subsection{Summary of Representation Theory Topics covered}

The field of morphological operator theory is a relatively new scientific field. It is our opinion that it essentially started in 1994 with Heijmans' monograph (Heijmans, 1994). Of course, there have been many original theoretical developments by several researchers before this, like the highly inspiring and more general books by Matheron (1975) and Serra (1982, 1988), but it is the first effort to systematically collect in a book the definitions and properties of most known morphological operators until 1994, both the classic and the advanced ones, and present them purely from a functional analysis viewpoint using the theoretical framework of operators on complete lattices (with the exception of a few elements from topology and metric spaces) without any focus on particular applications.

The part of this chapter on lattice-based morphology (Section 3) is written in a similar style as Heijman's monograph, which was actually published in the same series of Academic Press: Advances in Imaging and Electron Physics.

Section 2 summarizes some main concepts and results from linear operator theory, both from an algebraic viewpoint (structure of linear vector spaces) as well as from a topological viewpoint (normed spaces) and a geometric viewpoint (inner product spaces). It also gives examples of finiteand infinite-dimensional linear spaces related to image and signal processing. This material is classic and can be found in numerous books; our exposition follows mainly Naylor and Sell (1982) and in some cases Royden (1968). A subtopic we emphasize is that about linear projections and the projection theorem in Hilbert spaces. Another major topic is Riesz's representation theorem where we explain how it leads to the classic result of linear signal processing that every stable linear and shift-invariant filter is a linear convolution with its impulse response. Another closely related topic is the matrix representation of linear operators over finite-dimensional vector spaces. We conclude this section with the spectral representation of linear operators over finite-dimensional spaces using projections.

Section 3 begins with a synopsis of partially ordered sets (posets) and various aspects of complete lattices from Birkhoff (1967), including sublattices, atoms, Boolean and function lattices. Then, we provide examples of image lattices. Afterwards, we summarize the definitions and properties of the main types of lattice operators (Serra, 1988; Heijmans and Ronse, 1990; Ronse and Heijmans, 1991), and talk about the monotone case especially about dilations as operators that distribute over any suprema and erosions as operators that distribute over infima. Examples are given from the classic (Euclidean) morphological operators to show compatibility with their lattice generalizations. The main focus and details in this section are given to three subtopics: (1) the lattice adjunctions, which are pairs of compatible lattice erosions and dilations generating openings and 
closings, (2) the spatially-varying lattice operations with a structuring element map which create a variety of adaptive morphological operators, and (3) the lattice projections, which are increasing and idempotent filters that include the classic openings, closings, their lattice generalizations (e.g. radial, area, and reconstruction filters), and their non-(anti)-extensive combinations. Finally, as an aside, we mention issues of convergence and continuity for lattice operators, mainly related to their order structure.

Section 4 extends the lattice theory of mathematical morphology from the viewpoints of minimax algebra and translation-invariant systems by introducing a nonlinear signal space that has both a complete lattice structure, i.e. partial order, and sup/inf superpositions of signals from arbitrary (possibly infinite) collections, as well as two scalar 'multiplication'-type operations ( and its dual $\star^{\prime}$ ) that distribute over any suprema and infima, respectively. The scalar arithmetic in this nonlinear space of signals or vectors is based on the clodum structure. Thus, the linear superposition of signals over a linear spaces is replaced now by a max- $\star$ combination in the finitedimensional or sup- $\star$ in the infinite-dimensional case and its dual inf- $\star^{\prime}$ variant. We propose that the resulting nonlinear signal space is called a complete weighted lattice. The minimax vector spaces in Cuninghame-Green (1979) are a special finite-dimensional case of complete weighted lattices. In this chapter we introduce a set of axioms for defining their algebraic structure. Their role for analyzing lattice operators is conceptually similar to the role played by linear (sum-product) vector spaces for linear operators.

This generalized signal algebra unifies the following algebraic structures: (1) Max-Plus mathematical morphology: classic Minkowski translation-invariant operators, i.e. max-plus convolutions. (2) Max-Product mathematical morphology: Lattice extensions of Minkowski dilations and erosions with multiplicative structuring elements. (3) Fuzzy image operations and convolutions, where $\star$ $\left(\star^{\prime}\right)$ becomes a fuzzy intersection (union).

One of the consequences of this unified view, which is related to the central theme of this chapter, is the ability to represent and analyze adaptive or translation-invariant operators that are compatible with this generalized algebraic structure via generalized sup- $\star$ convolutions. In the adaptive case we have spatially-varying convolutions by instances of an impulse response map. In the translation-invariant case we obtain operations whose computational structure is like that of linear convolutions but the linear sum-of-products has been replaced by sup-of- $\star$ or inf-of- $\star^{\prime}$ operations. In the finite-dimensional case, we show that any operator that distributes over the main operations of this nonlinear space admits a matrix representation where the output vector is the generalized max- $\star$ (or its dual min- $\star^{\prime}$ ) 'product' of the operator matrix with the input vector.

Section 5 presents a unified representation theory to decompose an operator into a parallel combination (sup/inf superposition) of elementary morphological operators. We classify below the representations according to the required properties of the operators to be represented. (i) We begin with the most well-studied class of lattice operators: increasing and translation-invariant (TI) operators for sets or signals defined on a Euclidean domain $\mathbb{R}^{m}$ or its discrete version $\mathbb{Z}^{m}$. The first major representation theorem (Matheron, 1975) concerns increasing and translation-invariant (TI) set operators based on their kernel, a special collection of inputs which is capable of uniquely synthesizing the operator as union of erosions by its kernel sets of as an intersection of dilations by sets in the kernel of its dual operator. The second major step (Maragos, 1985) is to find a minimal (irreducible) representation that uses only the basis of the operator, which is defined as the collection of minimal kernel elements, by adding an extra constraint on the operator to be semi-continuous. Then follows the extension to increasing TI signal operators based both on their kernel as well as on their basis (Maragos, 1985, 1989a). The above were exact representations. We also discuss approximate representations using a truncated basis. (ii) If we remove the operator constraint/property of being increasing, we are left with a non-monotone TI operator, but it is still possible to represent it as union of simpler non-monotone operations of the hit-miss type by using the set intervals in the kernel or in a smaller (basis-like) collection consisting of the maximal kernel intervals (Banon and Barrera, 1991). In practical applications of representations of type (i) or (ii), of interest are also approximate representations by using a truncated basis 
and quantifying the relative error (Loce and Dougherty, 1992a,b, 1995). (iii) If we now remove the TI property from the operator and leave only the increasingness, we have spatially-varying operators for which similar representations have been developed as sup/inf combinations of adaptive erosions/dilations (Bouaynaya et al., 2008; Bouaynaya and Schonfeld, 2008). In Section 5 we survey the morphological presentations for all the above three cases of operators. In addition, we provide many examples of applying the representation theorems to various classes of nonlinear systems (morphological filters, rank/median filters, stack filters, window operators for shape detection) and linear systems (digital linear FIR filters); references will be given in the corresponding subsections. All the above representations assumed either the framework of Euclidean or lattice morphology. (iv) Continuing in this section, we describe kernel representations for increasing and group-invariant operators on complete weighted lattices based on initial ideas from (Maragos, 2005a). (v) We also investigate a class of set operators that combines four major properties: TI, increasing, idempotent and anti-extensive, i.e. general TI set openings. We connect their representation as union of Minkowski openings (Matheron, 1975) by sets in a subcollection of their invariant sets with their representation as union of erosions by their basis sets. Finally, references are also provided for extensions of the above representations (i), (ii), (iii) and (v) in a more abstract form to general lattice operators or general domains where the Euclidean group of translations is replaced by other groups of automorphisms (Serra, 1988; Heijmans and Ronse, 1990; Ronse and Heijmans, 1991; Banon and Barrera, 1993).

\subsection{Notation}

For linear operators, we denote with lowercase roman letters the elements (e.g. vectors or signals) of linear spaces and the scalars, whereas linear spaces and linear operators are denoted by capital roman letters.

For morphological operators, we use lowercase roman letters for points in sets, capital roman letters for sets, calligraphic capital roman letters for collection of sets or nonlinear spaces, and greek letters for operators.

We use boldface roman letters for vectors (lowercase) and matrices (capital) only when there is a vector or matrix operation, or to denote a point in the multidimensional Euclidean space $\mathbb{R}^{m}$ or discrete space $\mathbb{Z}^{m}$ when this serves as an image domain on which we can define the standard vector operations (vector addition, multiplication of a vector by a scalar, horizontal vector translations).

In general, for the morphological operators examined in this chapter, we follow the notation of Sternberg (1986) and Maragos and Schafer (1990) for the classic (Minkowski-type, translationinvariant) operators, whereas we follow the notation of Heijmans (1994) for the lattice operators. So for the image operators we use a different notation than the one used in image algebra (Ritter and Wilson, 2001). Also, for the complete weighted lattices and the related max-plus arithmetic and matrix algebra we use a different notation than the one used in minimax algebra (CuninghameGreen, 1979); in this case our notation is more consistent with morphological symbols and lattice operators.

\section{Linear Spaces and Linear Image Operators}

Most of the material in this section follows Naylor and Sell (1982); some discussion on linear spaces is also influenced by Royden (1968).

\subsection{Linear Spaces}

We shall outline some basic ideas in linear spaces from three viewpoints:

- Only algebraic structure, common to all linear spaces.

- Combined topological and algebraic structure by having a norm, in Banach spaces. 
- Combined geometric and algebraic structure by having an inner-product, in Hilbert spaces.

\subsubsection{Algebraic Structure}

A nonempty set $V$ of mathematical objects (usually called 'vectors') is called a linear space over a field $F$ of scalar numbers (e.g. the field of real or complex numbers) if we can define an addition operation $x+y$ for elements ('vectors') $x, y$ of the underlying set $V$ and a scalar multiplication $a x$ of 'vectors' $x$ by scalars $a$ such that:

(1). $V$ becomes a commutative group under vector addition, and

(2). The scalar multiplication of vectors in $V$ distributes over the vector addition in a similar way as in $F$ the scalar field multiplication distributes over the scalar field addition.

In more details, $V$ is a linea space if its operations satisfy the following axioms for all $x, y, z \in V$ and all $a, b \in F$ :

(A1). $x+y \in V$

(A2). $x+y=y+x$

(A3). $x+(y+z)=(x+y)+z$

(A4). $\exists \mathbf{0} \in V$ such that $x+\mathbf{0}=x$

(A5). $\exists(-x) \in V$ such that $x+(-x)=0$

(SM1). $a x \in V$

(SM2). $a(b x)=(a b) x$

(SM3). $1 x=x$, where 1 is the unit of $F$

(SM4). $0 x=\mathbf{0}$, where 0 is the zero of $F$

$(\mathrm{A} \& \mathrm{SM} 1) \cdot a(x+y)=a x+a y$

(A\&SM2). $(a+b) x=a x+b x$
(Closure under vector addition) (Commutativity of vector addition) (Associativity of vector addition)

(Existence of addition identity)

(Existence of addition inverse) (Closure under scalar multiplication) (Associativity of scalar multiplication)

(Multiplication with scalar unit)

(Multiplication with scalar zero)

(Distributivity 1)

(Distributivity 2)

Not all the above axioms are logically independent.

Often, a linear space is also called a vector space and its set elements are called 'vectors'. However, linear spaces can be spaces of vectors, or signals, or functionals, or signal operators, or other general objects. A linear space over the field of real or complex numbers is called a real or complex linear space, respectively.

A subset $S$ of a linear space $V$ is called a linear subspace if it is itself a linear space over the same scalar field $F$. This is equivalent to $S$ being closed under addition and scalar multiplication.

Consider a (possibly infinite) subset $A$ of a linear space $V$. A space element $x \in V$ is called a linear combination of points in $A$ if there exists a finite set of elements $\left\{x_{1}, x_{2}, \ldots, x_{n}\right\}$ in $A$ and a finite set of scalars $\left\{r_{1}, r_{2}, \ldots, r_{n}\right\}$ such that

$$
x=r_{1} x_{1}+r_{2} x_{2}+\cdots+r_{n} x_{n}
$$

The linear span of $A$, denoted by $\operatorname{span}(A)$, is the set of all linear combinations of elements in $A$. If $A=\varnothing$, by convention we set $\operatorname{span}(A)=\{\boldsymbol{0}\}$. The linear span of any $A \subseteq V$ is a linear subspace of $V$, and actually the smallest subspace containing $A$.

A set $S$ in a linear space $X$ is called linearly independent if each point $v \in S$ is not a linear combination of points in $S \backslash\{v\}$. Equivalently, $S$ is linearly independent if and only if (abrreviated as 'iff') for each nonempty finite subset of $S$, e.g. $\left\{x_{1}, x_{2}, \ldots, x_{n}\right\}$, the only scalars satisfying the equation

$$
a_{1} x_{1}+a_{2} x_{2}+\cdots+a_{n} x_{n}=\mathbf{0}
$$

are $a_{1}=\cdots=a_{n}=0$. The set $S$ is called linearly dependent if it contains at least one point $v$ that is a linear combination of points in $S \backslash\{v\}$.

Theorem 1 Let $S$ be a nonempty subset of a linear space $X$. Then, for each $v \neq \mathbf{0}$ that belongs to the linear span of $S$, there is a unique $n$-tuple $\left(x_{1}, x_{2}, \ldots, x_{n}\right)$ of points in $S$ and a unique $n$-tuple of nonzero scalars $\left(a_{1}, \ldots, a_{n}\right)$ such that

$$
v=a_{1} x_{1}+a_{2} x_{2}+\cdots+a_{n} x_{n}
$$


A Hamel basis of $X$ is a set $B \subseteq X$ that is linearly independent and its linear span makes up all the space $V$. Every linear space $X$ has a Hamel basis. However, all possible Hamel bases of a space have the same cardinality. This common cardinality of any Hamel basis determines the dimension of the space, denoted $\operatorname{dim}(X)$. If it has a finite basis, the space is called finitedimensional. Otherwise the linear space is called infinite-dimensional.

\subsubsection{Topological Structure, Banach spaces}

A real-valued function $\|\cdot\|$ defined on a linear space $V$ over $F$ is called a norm if for all $x, y \in V$ and $a \in F$ :

(N1) $\|x\| \geq 0$ and $\|\mathbf{0}\|=0$

(Positivity)

(N2) $\|x+y\| \geq\|x\|+\|y\|$

(Triangle inequality)

(N3) $\|a x\|=|a|\|x\|$

(Homogeneity)

(N4) $\|x\|=0 \Longrightarrow x=\mathbf{0}$

(Strict Positivity).

If the function satisfies only (N1), (N2) and (N3), it is called a seminorm (or pseudonorm).

A linear space equipped with a norm becomes a normed linear space. Each normed space is a metric space, where the metric is defined in terms of the given norm by $d(x, y)=\|x-y\|$. In such spaces, the axioms for defining a linear space determine its algebraic structure, whereas the norm endows it with topological and metrical properties. Thus, normed spaces possess some weak geometric structure since, in addition to vector structure, they have distances and lengths.

Two norms $\|\cdot\|_{a}$ and $\|\cdot\|_{b}$ on a linear space $X$ are called equivalent if they generate equivalent metrics, i.e. create the same topology on $X$. It can be shown that the two norms are equivalent iff there exist positive constants $c_{1}$ and $c_{2}$ such that for all $x \in X$

$$
c_{1}\|x\|_{a} \leq\|x\|_{b} \leq c_{2}\|x\|_{a}
$$

On a finite-dimensional linear space, two norms are always equivalent. A normed linear space $(X,\|\cdot\|)$ is finite-dimensional iff the unit ball $\{x \in X:\|x\| \leq 1\}$ is a compact set.

Let $X$ be a normed linear space. A sequence $\left(x_{n}\right)$ in $X$ is called convergent if there is $x \in X$ such that $\left\|x_{n}-x\right\| \rightarrow 0$ as $n \rightarrow \infty$; we write $\lim x_{n}=x$. If this sequence limit exists, it is unique. A sequence $\left(x_{n}\right)$ is called Cauchy sequence if $\left\|x_{n}-x_{m}\right\| \rightarrow 0$ as $n, m \rightarrow \infty$. If each Cauchy sequence in $X$ converges to a point in $X$, then $X$ is called complete. A complete normed linear space is called a Banach space.

To analyze an infinite series $\sum_{n=1}^{\infty} x_{n}$ in $X$, from its term sequence $\left(x_{n}\right)$ we form the sequence of partial sums $y_{m}=\sum_{n=1}^{m} x_{n}$. If $\left(y_{m}\right)$ converges to a limit $y \in X$, we say that the infinite series converges and write

$$
y=\sum_{n=1}^{\infty} x_{n}
$$

In a Banach space $X$, testing the convergence of the above series becomes simple via the Cauchy test which checks whether the sequence of partial sums is a Cauchy sequence. Stronger types of convergence for the infinite series are: (i) absolute convergence where the real series $\sum_{n=1}^{\infty}\left\|x_{n}\right\|$ converges and (ii) unconditional convergence where all the series resulting by all possible permutations of the series terms converge and have the same limit. Obviously, unconditional convergence implies simple convergence. In a Banach space $X$, if a series is absolutely convergent, then it is also unconditionally convergent. The converse is true if $X$ is finite-dimensional.

\subsubsection{Geometric Structure, Hilbert spaces}

An even closer resemblance to Euclidean geometry exists in inner product linear spaces, which are complex or real linear spaces on which a mapping from $V \times V$ to $F$ is defined (where $F$ is $\mathbb{C}$ or $\mathbb{R}$ ), called inner product, that is denoted by $\langle\cdot, \cdot\rangle\rangle$ and satisfies the following axioms: for all $x, y, z \in V$ and $a \in F$,

(IP1) $\langle x+y, z\rangle=\langle x, z\rangle+\langle y, z\rangle$ 
(IP2) $\langle a x, y\rangle=a\langle x, y\rangle$

(Homogeneity)

(IP3) $\langle x, y\rangle=\langle y, x\rangle^{*}$

(Symmetry)

(IP4) $\langle x, x\rangle>0$ if $x \neq 0$

(Positive Definiteness)

where $(\cdot)^{*}$ denotes complex conjugate. The first three axioms imply the following additional properties:

(IP5) $\langle x, y+z\rangle=\langle x, y\rangle+\langle x, z\rangle$

(IP6) $\langle x, a y\rangle=a^{*}\langle x, y\rangle$.

A (complex or real) Hilbert space is a (complex or real) complete inner product linear space.

Any inner product generates a norm defined as follows:

$$
\|x\| \triangleq \sqrt{\langle x, x\rangle}
$$

Lemma 1 (Cauchy-Schwarz Inequality). For any elements $x, y$ of an inner product space,

$$
|\langle x, y\rangle| \leq\|x\|\|y\|
$$

Equality holds iff $x$ and $y$ are linearly dependent.

In an inner product space $X$, two elements $x, y$ are called orthogonal, written as $x \perp y$, if $\langle x, y\rangle=0$. Two subsets $A, B$ of $X$ are called orthogonal, written as $A \perp B$, if $\langle x, y\rangle=0$ for all $(x, y) \in A \times B$.

Theorem 2 (Generalized Pythagorean Theorem). In an inner product space, if two elements $x, y$ are orthogonal, then

$$
\|x+y\|^{2}=\|x\|^{2}+\|y\|^{2}
$$

The converse is true if the space is real.

\subsubsection{Examples of Linear Image Spaces}

Finite-dimensional Spaces: The simplest image function model is a finite vector $\mathbf{f}=\left(f_{1}, \ldots, f_{N}\right)$ of $N$ real- or complex-valued image samples formed by some indexing of the finite image domain. If our application or problem requires linear operations, then the image space is the finite-dimensional vector space $\left(F^{N},\|\cdot\|_{p}\right)$, where $F$ is $\mathbb{R}$ or $\mathbb{C}$, equipped with the familiar $\ell^{p}$ norms for vectors:

$$
\|\mathbf{f}\|_{p} \triangleq \begin{cases}\left(\left|f_{1}\right|^{p}+\left|f_{2}\right|^{p}+\cdots+\left|f_{N}\right|^{p}\right)^{1 / p}, & 1 \leq p<\infty \\ \max \left(\left|f_{1}\right|,\left|f_{2}\right|, \ldots,\left|f_{N}\right|\right), & p=\infty\end{cases}
$$

For each $p \geq 1$, we have a Banach space. The case $p=2$ corresponds to the well-known Euclidean norm $\|\mathbf{f}\|=\sqrt{\langle\mathbf{f}, \mathbf{f}\rangle}$, which yields a Hilbert space with the familiar inner-product

$$
\langle\mathbf{f}, \mathbf{g}\rangle=\mathbf{f} \cdot \mathbf{g}=f_{1} g_{1}{ }^{*}+f_{2} g_{2}{ }^{*}+\cdots+f_{N} g_{N}{ }^{*}
$$

Since all the $\ell^{p}$ norms are equivalent, we generally refer to the linear spaces $\left(F^{N},\|\cdot\|_{p}\right)$ as Euclidean spaces.

Discrete-domain Infinite-dimensional Image Spaces: Consider the complex or real linear space $\ell\left(\mathbb{Z}^{m}\right)$ that consists of all complex- or real-valued $m$-dimensional infinite sequences denoted as discrete-domain signals $f[\mathbf{n}]$ with $\mathbf{n}=\left(n_{1}, \ldots, n_{m}\right) \in \mathbb{Z}^{m}, m=1,2,3, \ldots$, where vector addition and scalar multiplication is, respectively, the pointwise sum of two sequences and the multiplication of a sequence by a scalar. The $\ell^{p}$ norm of $f[\mathbf{n}]$ is defined by

$$
\|f\|_{p} \triangleq \begin{cases}\left(\sum_{\mathbf{n} \in \mathbb{Z}^{m}}|f[\mathbf{n}]|^{p}\right)^{1 / p}, & 1 \leq p<\infty \\ \sup _{\mathbf{n} \in \mathbb{Z}^{m}}|f[\mathbf{n}]|, & p=\infty\end{cases}
$$


The subset of image sequences with finite norm is a linear subspace because it is closed under scalar multiplication and vector addition. The latter results from the Minkowski Inequality:

$$
\|f+g\|_{p} \leq\|f\|_{p}+\|g\|_{p}, \quad 1 \leq p \leq \infty
$$

for any two sequences $f, g$. For each $p \in[1, \infty]$, this subspace of $m$-dimensional infinite sequences with finite $\ell^{p}$ norm is a Banach space called the $\ell^{p}\left(\mathbb{Z}^{m}\right)$ space. Three cases are most important for the analysis of discrete-domain images: for $p=1$ we have the space of absolutely summable images, whereas for $p=\infty$ we have the space of bounded image sequences. Finally, for $p=2$ we have the space of finite-energy image sequences, which is a Hilbert space with inner product given by

$$
\langle f, g\rangle=\sum_{\mathbf{n} \in \mathbb{Z}^{m}} f[\mathbf{n}] g^{*}[\mathbf{n}]
$$

Hölder Inequality: For $1 \leq p \leq \infty$ and $p^{-1}+q^{-1}=1$,

$$
\sum_{\mathbf{n} \in \mathbb{Z}^{m}}\left|f[\mathbf{n}] g^{*}[\mathbf{n}]\right| \leq\|f\|_{p} \cdot\|g\|_{q}
$$

Continuous-domain Infinite-dimensional Image Spaces: Consider all real- or complexvalued image functions defined on a measurable (e.g. open and usually bounded) subset $E$ of $\mathbb{R}^{m}$ which are measurable ${ }^{3}$ and Lebesgue integrable, i.e. $\int_{E}\|f\|<\infty$. Their collection forms a linear space, $L^{1}(E, \mathbb{C})$, under function addition and scalar multiplication. More generally, for $1 \leq p<\infty$, let $L^{p}(E, \mathbb{C})$ be the linear space of all those functions whose $p$-th absolute power is integrable, i.e. whose $L^{p}$ norm is finite:

$$
\|f\|_{L^{p}}=\left(\int_{E}|f|^{p}(\mathbf{x}) d \mathbf{x}\right)^{1 / p}<\infty
$$

We may omit the range and image domain from the notation of $L^{p}$ if understood from the context. In $L^{p}$, if two functions $f$ and $g$ are equal almost everywhere (a.e.), i.e. $A=\{\mathbf{x}: f(\mathbf{x}) \neq g(\mathbf{x})\}$ has measure zero, then $\int_{E} f=\int_{E} g$ and hence $\|f-g\|_{L^{p}}=0$. Thus, $\|f\|_{L^{p}}=0$ means that $f=0$ a.e. Hence, the function $\|\cdot\|_{L^{p}}$ is not a norm, but a seminorm. However, we can make it a norm by defining it on equivalence classes of functions where two functions are equivalent if they are equal a.e. Thus, if we don't distinguish between two equivalent functions, then $\|\cdot\|_{L^{p}}$ becomes a norm, and $L^{p}$ becomes a Banach space. For $p=2$, this is a Hilbert space with inner product

$$
\langle f, g\rangle=\int_{E} f(\mathbf{x}) g^{*}(\mathbf{x}) d \mathbf{x}
$$

Consider now the linear space $L^{\infty}$ of all scalar-valued measurable functions on $E$ that are bounded. This becomes a Banach space if we equip it with the essential supremum norm:

$$
\|f\|_{\infty}=\inf \{B:|f(\mathbf{x})| \leq B \text { almost everywhere }\}
$$

For a sequence of functions $\left(f_{n}\right)$ in the $L^{p}$ spaces, in addition to its pointwise convergence to a limit function $f$, which means that $\lim f_{n}(\mathbf{x})=f(\mathbf{x})$ for almost all points $\mathbf{x} \in E$, we more often use another type of convergence in the norm, denoted by $f_{n} \rightarrow f$ which means that $\lim _{n}\left\|f_{n}-f\right\|_{L^{p}}=0$. If $1 \leq p<\infty$, the norm convergence is referred to as convergence in the mean of order $p$. In the case $p=\infty$ the norm convergence is equivalent to uniform convergence a.e.

\footnotetext{
${ }^{3} \mathrm{~A}$ real-valued function is measurable if all its level sets are measurable. A complex-valued function is measurable if both its real and imaginary part are measurable functions.
} 
Spaces of Continuous and Differentiable Functions Consider the collection of real-valued continuous functions defined on some open set $E \subseteq \mathbb{R}^{m}$. Several subcollections of such functions form linear spaces that are of interest in image analysis and computer vision. Examples include the following:

1. The space $B C(E, \mathbb{R})$ of all bounded and continuous functions $f: E \rightarrow \mathbb{R}$ equipped with the supremum norm

$$
\|f\|_{\infty}=\sup \{f(\mathbf{x}): \mathbf{x} \in E\}
$$

is a Banach space. Further, if $E$ is compact, then the above space becomes the Banach space $C(E, \mathbb{R})$ of continuous real functions on $E$.

2. Space $L C(E, \mathbb{R})$ of Lipschitz continuous functions on $E$, i.e. functions $f$ for which there exists a constant $K>0$ (called Lipschitz coefficient) such that for all $\mathbf{x}, \mathbf{y} \in E$

$$
|f(\mathbf{x})-f(\mathbf{y})| \leq K\|\mathbf{x}-\mathbf{y}\|
$$

Let $\operatorname{Lip}(f)$ be the smallest constant $K$ satisfying (11); then $\operatorname{Lip}(f)$ is a seminorm. However the following is a norm:

$$
\|f\|_{L C}=\|f\|_{\infty}+\operatorname{Lip}(f)
$$

3. Space $C_{c}^{n}(E)$ of continuously differentiable functions up to order $n=0,1,2, \ldots$ and with compact support. A frequently used norm in this space is the square root of the sum of the square energies of the function and all its derivatives up to order $n$. The case $n=\infty$ is called the space of infinitely differentiable functions with compact support $C_{c}^{\infty}(E)=\bigcap_{n>0} C_{c}^{n}(E)$.

In examples 1 and 2 the domain $E$ may also generalize to an arbitrary metric space. Further, in all examples above the images may also be complex-valued.

\section{$2.2 \quad$ Linear Operators}

\subsubsection{Algebraic Definitions for Linear Operators}

A mapping $L: X \rightarrow Y$ between two linear spaces over the same scalar field is called a linear operator if it preserves addition and scalar multiplication. This is equivalent to

$$
L\left(a_{1} x_{1}+\cdots+a_{n} x_{n}\right)=a_{1} L\left(x_{1}\right)+\cdots+a_{n} L\left(x_{n}\right)
$$

for all $x_{1}, \ldots, x_{n} \in X$, all scalars $a_{1}, \ldots, a_{n}$ and all finite $n$. An operator that does not satisfy the above is called nonlinear. The null space and range of $L$ are defined as

$$
\operatorname{Nul}(L) \triangleq\{x \in X: L(x)=\mathbf{0}\}, \quad \operatorname{Ran}(L) \triangleq\{L(x): x \in X\}
$$

The null space is a linear subspace of $X$, whereas the range is a linear subspace of $Y$. For any linear operator $L: X \rightarrow Y$ between two linear spaces, the dimensions of its null and range spaces are related as follows to the dimension of the domain space:

$$
\operatorname{dim}\{\operatorname{Nul}(L)\}+\operatorname{dim}\{\operatorname{Ran}(L)\}=\operatorname{dim}\{\operatorname{Dom}(L)\}
$$

Two linear spaces $X$ and $Y$ over the same scalar field are called isomorphic if there exists an invertible linear operator $L: X \rightarrow Y$. Such a mapping $L$ is called an isomorphism between the two linear spaces. The inverse mapping $L^{-1}: Y \rightarrow X$ is also a linear operator. It can be shown that a linear operator $L$ is an isomorphism iff $\operatorname{Nul}(L)=\{\mathbf{0}\}$.

All linear spaces over the same field are isomorphic iff they have the same dimension. Hence, all real (resp. complex) finite-dimensional linear spaces are isomorphic to $\mathbb{R}^{n}$ (resp. $\mathbb{C}^{n}$ ) for some $n$. Thus, finite-dimensional linear spaces are essentially linear vector spaces, if by 'vector' ${ }^{4}$ we agree to mean a finite tuple of scalars.

\footnotetext{
${ }^{4}$ In the broad mathematics literature, the terminology 'vector space' is used as synonymous with 'linear space' and includes both finite- and infinite-dimensional linear spaces. In this chapter, since we are dealing with a large variety of linear and nonlinear spaces, we distinguish between the two terms and use the term 'vector space' only in the finite-dimensional case.
} 


\subsubsection{Linear Operators on Normed Spaces}

A map $f: X \rightarrow Y$ between two normed linear spaces is called continuous if $\lim f\left(x_{n}\right)=f(x)$ for each convergent sequence $x_{n} \rightarrow x$ in $X$, or equivalently if $\lim \left\|f\left(x_{n}\right)-f(x)\right\|_{Y}=0$ whenever $\lim \left\|x_{n}-x\right\|_{X}=0$. Continuous linear maps are the only ones distributing with infinite series as the following fundamental result implies.

Theorem 3 (Principle of Infinite Linear Superposition). Let $L: X \rightarrow Y$ be a mapping between two normed linear spaces. Then, $L$ is a continuous linear operator if and only if

$$
L\left(\sum_{n=1}^{\infty} a_{n} x_{n}\right)=\sum_{n=1}^{\infty} a_{n} L\left(x_{n}\right)
$$

for every convergent series $\sum_{n=1}^{\infty} a_{n} x_{n}$.

An operator $L: X \rightarrow Y$ between two normed linear spaces is called bounded if there exists $M \geq 0$ such that $\|L(x)\| \leq M\|x\|$ for all $x \in X$. Equivalently, $L$ is bounded iff its operator norm, defined as

$$
\|L\| \triangleq \inf \{M \geq 0:\|L(x)\| \leq M\|x\| \forall x\}
$$

is finite. More versatile expressions for the operator norm are the following:

$$
\begin{aligned}
\|L\| & =\sup \{\|L(x)\| /\|x\|: x \neq \mathbf{0}\} \\
& =\sup \{\|L(x)\|:\|x\| \leq 1\}
\end{aligned}
$$

Intuitively, $\|L\|$ expresses the 'maximum' gain of the operator. Obviously,

$$
\|L(x)\| \leq\|L\| \cdot\|x\|, \quad \forall x \in X .
$$

Theorem 4 A linear transformation between two normed linear spaces is continuous if and only if it is bounded.

Space of Bounded Linear Operators: The collection $B L T(X, Y)$ of all bounded linear transformations $L: X \rightarrow Y$ between two normed linear spaces equipped with the above operator norm $\|L\|$ becomes a normed linear space, where the operator addition and scalar multiplication are induced by the corresponding operations on the operators' inputs and outputs. This operator space is Banach if $Y$ is Banach. For the norm of the composition of two operators $S, T$ in this space we have

$$
\|S T\| \leq\|S\| \cdot\|T\|
$$

If we need to analyze the limiting behavior of an infinite sequence of operators in $B L T(X, Y)$, we need to study its convergence. This can be done in two ways. 1) Globally via the operator norm: We say that a sequence $\left(T_{n}\right)$ converges uniformly to $T$ in $B L T(X, Y)$, written as $\lim T_{n}=T$, if $\lim \left\|T_{n}-T\right\|=0$. 2) Locally at each point: The sequence $\left(T_{n}\right)$ converges strongly to $T$ in $B L T(X, Y)$, written as $\lim T_{n}={ }_{s} T$, if $\lim \left\|T_{n}(x)-T(x)\right\|=0$ for all $x \in X$. Uniform convergence implies strong convergence.

\subsubsection{Linear Operators on Inner Product Spaces}

Let $L: H \rightarrow K$ be a bounded linear operator between two Hilbert spaces. Then there exists a unique operator $L^{*}: K \rightarrow H$, called the adjoint of $L$, such that for all $x \in H, y \in K$

$$
\langle L(x), y\rangle_{K}=\left\langle x, L^{*}(y)\right\rangle_{H}
$$

A bounded linear operator $T$ on a Hilbert space is called normal if it commutes with its adjoint: $T T^{*}=T^{*} T$. A self-adjoint operator is a special case of a normal operator $S$ with $S=S^{*}$.

Some of the properties of the adjoint include the following: 
Proposition 1 Let $L, S, T$ be bounded linear operators on a Hilbert space $H$. Then:

(a) The adjoint $L^{*}$ of $L$ is itself a bounded linear operator with $\left\|L^{*}\right\|=\|L\|$.

(b) $\left(L^{*}\right)^{*}=L^{*}$.

(c) $(S+T)^{*}=S^{*}+T^{*}$ and $(a L)^{*}=a^{*} L^{*}$.

(d) $(S T)^{*}=T^{*} S^{*}$.

(e) $S$ is self-adjoint iff $\langle x, S(x)\rangle \in \mathbb{R}$ for all $x \in H$.

A self-adjoint operator $S$ is called positive, written as $S \geq 0$, if $\langle x, S(x)\rangle \geq 0$ for all $x \in H$ and strictly-positive if $\langle x, S(x)\rangle>0$ for all $x \neq \mathbf{0}$. The following introduces a partial ordering on the class of self-adjoint operators $S, T$ :

$$
S \leq T \Longleftrightarrow 0 \leq T-S
$$

Examples 1 (Self-Adjoint and Normal Operators):

(a) The identity $I$ and zero operator $O$ are self-adjoint.

(b) If a finite-dimensional linear operator $A$ on $\mathbb{C}^{n}$ is represented by a matrix $\mathbf{A}$, its adjoint corresponds to the matrix $\mathbf{A}^{H}$. The operator is self-adjoint iff $\mathbf{A}$ is Hermitian. Further, the operator is positive iff the matrix is positive semidefinite. More generally, the operator is normal iff the matrix is square and $\mathbf{A} \mathbf{A}^{H}=\mathbf{A}^{H} \mathbf{A}$.

(c) Bounded linear time-invariant operators are normal.

(d) A continuous projection on a Hilbert space $H$ is orthogonal iff it is self-adjoint.

\subsubsection{Isomorphisms}

There are four ways that linear spaces $X$ and $Y$ can be isomorphic in the sense of having equivalent algebraic structure and geometric structure (if the latter exists). Next we define these types of isomorphisms in order of increasing structure; namely each new definition makes the assumptions of the previous ones. The last three types are bounded linear operators.

Specifically two linear spaces $X$ and $Y$ over the same field are called:

- (Algebraically) Isomorphic if there exists a linear bijection $\alpha: X \rightarrow Y$. Such a map is called an (algebraic) isomorphism of $X$ onto $Y$.

- Topologically Isomorphic if they are normed spaces $\left(X,\|\cdot\|_{X}\right)$ and $\left(Y,\|\cdot\|_{Y}\right)$ for which there exists a linear bijection $\tau: X \rightarrow Y$ that is continuous and has a continuous inverse; $\tau$ is called a topological isomorphism. It can be proven that two linear normed spaces $\left(X,\|\cdot\|_{X}\right)$ and $\left(Y,\|\cdot\|_{Y}\right)$ are topologically isomorphic iff there exists a linear map $\phi: X \rightarrow Y$ and two positive constants $c_{1}, c_{2}$ such that for all $x \in X$

$$
c_{1}\|x\|_{X} \leq\|\phi(x)\|_{Y} \leq c_{2}\|x\|_{X}
$$

The map $\phi$ satisfying (15) is a topological isomorphism.

- Isometrically Isomorphic if they are normed spaces for which there exists a linear map $\mu: X \rightarrow Y$ that preserves norms:

$$
\|\mu(x)\|_{Y}=\|x\|_{X}, \quad \forall x \in X
$$

$\mu$ is called an isometry isomorphism, or simply a linear isometry. Note that (16) implies that $\mu$ is continuous and that $\mu^{-1}$ exists and is a linear isometry.

- Unitarily Equivalent if they are inner-product spaces $\left(X,\langle\cdot\rangle_{X}\right)$ and $\left(Y,\langle\cdot\rangle_{Y}\right)$ for which there exists a linear bijection $\theta: X \rightarrow Y$ that preserves inner products:

$$
\langle\theta(x), \theta(y)\rangle_{Y}=\langle x, y\rangle_{X}, \quad \forall x, y \in X
$$

$\theta$ is called a unitary operator. Note that a linear map between two inner-product spaces is unitary iff it is an isometry. 


\subsection{Linear Projections}

\subsubsection{Algebraic Decomposition}

Assume we have two linear subspaces $A$ and $B$ of a linear space $X$. There are two ways to combine them and form a new space. Their sum is defined as the linear space

$$
A \oplus B=\{a+b: a \in A, b \in B\}
$$

and their product is defined as the linear space

$$
A \times B=\{(a, b): a \in A, b \in B\}
$$

where addition and scalar multiplication is defined componentwise. ${ }^{5}$ Thus, the sum of the subspaces $A$ and $B$ equals the $\operatorname{span}(A \cup B)$, whereas their product has as underlying set their Cartesian product.

Two subspaces $A$ and $B$ of $X$ are called disjoint if $A \cap B=\{\mathbf{0}\}$. If additionally $X=A \oplus B$, then $B$ is called the algebraic complement of $A$. All algebraic complements of a subspace $A$ have the same dimension, called the co-dimension of $A$. The concept of algebraic complement offers a decomposition of a linear space $X$ into a sum of disjoint subspaces $A, B$ where each point in $X$ admits a unique expression as a sum of two points in $A$ and $B$, respectively.

Lemma 2 Two linear subspaces $A$ and $B$ of a linear space $X$ are algebraic complements of each other if and only if for each $x \in X$ there is a unique pair $\left(x_{1}, x_{2}\right) \in A \times B$ with $x=x_{1}+x_{2}$.

A projection $P: X \rightarrow X$ on a linear space $X$ is a linear map that is idempotent, i.e. $P^{2}=P$. A projection enables the decomposition of a linear space as in Lemma 2.

Theorem 5 (a) If $M$ and $N$ are two disjoint linear subspaces of a linear space $X$ such that $X=M \oplus N$, then there is a unique projection $P$ on $X$ with $M=\operatorname{Ran}(P)$ and $N=\operatorname{Nul}(P)$.

(b) Conversely, if $P$ is a projection, then its range and null space are algebraic complements of each other.

\subsubsection{Orthogonal Projections}

Consider an inner product space $X$. For any subset $M$ of $X$, its orthogonal complement is defined as

$$
M^{\perp} \triangleq\{x \in X:\langle x, y\rangle=0 \quad \forall y \in M\}
$$

We write $x \perp M$ if $x \in M^{\perp}$. If $M=\varnothing$, we set $M^{\perp}=X$. A projection $P$ on $X$ is called orthogonal if its range and null space are orthogonal sets. Orthogonal projections have many interesting properties.

Lemma 3 Let $P$ be an orthogonal projection on an inner product space $X$. Then:

(a) $P$ is a continuous, and hence bounded operator.

(b) If $P \neq O$, then $\|P\|=1$.

(c) Its range $M$ and null space $N$ are closed linear subspaces of $X$, which are also orthogonal and algebraic complements of each other.

(d) Each $x \in X$ can be written uniquely as $x=m+n$ with $(m, n) \in M \times N$ and $m \perp n$; hence, $\|x\|^{2}=\|m\|^{2}+\|n\|^{2}$.

\footnotetext{
${ }^{5}$ Several authors use a different notation: they call the sum of two linear subspaces $A$ and $B$ inner sum and denote it as $A+B$, whereas they call the product space as direct sum and denote it by $A \oplus B$. Our notation for $\oplus$ is the Minkowski set addition.
} 
Theorem 6 (Projection Theorem in Hilbert Spaces) Let $M$ be any closed subspace of a Hilbert space $H$. Then:

(a) There is a unique orthogonal projection $P$ on $H$ with $M=\operatorname{Ran}(P), M^{\perp}=\operatorname{Nul}(P)$, and $H=M \oplus M^{\perp}$.

(b) For each $x \in H$, its projection $y_{o}=P(x)$ is the unique point in $M$ whose difference from $x$ is orthogonal to $M$ :

$$
x-y_{o} \perp M
$$

(c) This unique point $y_{0}$ is at minimum distance from $x$ :

$$
\left\|x-y_{o}\right\|<\|x-y\| \forall y \in M \backslash\left\{y_{o}\right\}
$$

A direct corollary of this celebrated theorem is to interpret least squares approximation as simply an orthogonal projection. Thus, if we consider the points $y \in M$ as an approximation of the original data $x \in H$, then $y_{o}$ is the best linear approximation in the sense of minimizing the mean squared error (MSE), i.e. the squared norm $\mathcal{E}(x, y)=\|e\|^{2}$ of the approximation error $e=x-y$. This linear least squares approximation

$$
y_{o}=\operatorname{argmin}_{y \in M}\|x-y\|
$$

can be found easily as the orthogonal projection $y_{o}=P(x)$ of the original data onto $M$. Geometrically, the corresponding error $e_{o}=x-y_{o}$ is orthogonal to all points in $M$ and the minimum error norm is

$$
\mathcal{E}_{\min }(x)=\left\|e_{o}\right\|^{2}=\left\langle x, e_{o}\right\rangle=\|x\|^{2}-\langle x, P(x)\rangle
$$

If the subspace $M$ is finite-dimensional and is the span of $p$ linearly independent vectors $u_{1}, u_{2}, \ldots, u_{p}$, then the best approximation is a linear combination of these basis vectors:

$$
y_{o}=\sum_{k=1}^{p} a_{k} u_{k}
$$

Exploiting the orthogonality conditions $e_{o} \perp u_{k}$ gives rise to the normal equations

$$
\begin{aligned}
\sum_{k=1}^{p} a_{k}\left\langle u_{k}, u_{i}\right\rangle & =\left\langle x, u_{i}\right\rangle, \quad i=1, \ldots, p \\
\mathcal{E}_{\min } & =\|x\|^{2}-\sum_{k=1}^{p} a_{k}{ }^{*}\left\langle x, u_{k}\right\rangle
\end{aligned}
$$

which are the basic tool in computing the optimal coefficients and the minimum error for least squares approximation problems.

\subsection{Representations of Linear Operators}

\subsubsection{Matrix Representation of Linear Operators on Finite-dimensional Vector Spaces}

Let $L: X \rightarrow Y$ a linear operator between two finite-dimensional linear spaces $X$ and $Y$ whose Hamel bases are, respectively, the sets $B_{1}=\left\{x_{1}, \ldots, x_{n}\right\}$ and $B_{2}=\left\{y_{1}, \ldots, y_{m}\right\}$. Since every point $x$ in $X$ has a unique representation as linear combination of basis elements

$$
x=c_{1} x_{1}+\cdots+c_{n} x_{n}
$$

its image will be

$$
L(x)=c_{1} L\left(x_{1}\right)+\cdots+c_{n} L\left(x_{n}\right)
$$

Since $L(x)$ is a point in $Y$, it can be expressed as a unique linear combination of the basis elements of $Y$ :

$$
L(x)=d_{1} y_{1}+\cdots+d_{m} y_{m}
$$


In addition, $L\left(x_{1}\right), \ldots, L\left(x_{n}\right)$ are also points in $Y$ and can be expressed by corresponding unique linear combinations of the basis elements of $Y$ :

$$
\begin{array}{ccc}
L\left(x_{1}\right) & = & a_{11} y_{1}+\cdots+a_{m 1} y_{m} \\
\vdots & \vdots & \vdots \\
L\left(x_{n}\right) & = & a_{1 n} y_{1}+\cdots+a_{m n} y_{m}
\end{array}
$$

Hence, the two vectors of coefficients of the expansions of $x$ and $L(x)$ in the corresponding bases are related via a matrix-vector multiplication:

$$
[L]\left[\begin{array}{c}
c_{1} \\
c_{2} \\
\vdots \\
c_{n}
\end{array}\right]=\left[\begin{array}{c}
d_{1} \\
d_{2} \\
\vdots \\
d_{m}
\end{array}\right], \quad[L]=\left[\begin{array}{cccc}
a_{11} & a_{12} & \cdots & a_{1 n} \\
a_{21} & a_{22} & \cdots & a_{2 n} \\
\vdots & \vdots & \vdots & \vdots \\
a_{m 1} & a_{m 2} & \cdots & a_{m n}
\end{array}\right]
$$

Thus, there is one-to-one correspondence between the linear operator $L$ and the $m \times n$ matrix $[L]$ over the field $F$.

Similarly, if we consider a linear operator from $X$ to itself, then this corresponds to a square $n \times n$ matrix with elements from $F$. Let $F^{n \times n}$ the set of all such matrices; equipped with addition and multiplication of matrices, it becomes ring. Therefore, if we consider the set of all linear operators from $X$ to itself equipped under addition of composition of operators, this algebraic structure is a ring of linear operators, which is isomorphic to the matrix $\operatorname{ring} F^{n \times n}$.

\subsubsection{Riesz Representation Theorem and Linear Convolution}

Given a linear space $V$ over a field $F$ of scalars, a linear functional on $V$ is called any linear transformation of $V$ into its scalar field $F$, i.e. any linear operator $\ell: V \rightarrow F$ that maps points (e.g. vectors, signals) of the space to scalar numbers.

Theorem 7 (Riesz Representation TheOrem). Let $\phi$ be a bounded linear functional on a Hilbert space $H$. Then there exists a unique point $k \in H$ such that $\phi(x)=\langle x, k\rangle$ for all $x \in H$. Further, $\|\phi\|=\|k\|$.

The 'vector' $k$ is called a representation of the functional $\phi$; thus, a signal represents a linear system. This fundamental theorem is the basis of the convolutional representation of linear systems. Let $H$ be the Hilbert space $L^{2}(\mathbb{R}, \mathbb{C})$ of complex-valued continuous-time signals. Consider a bounded linear operator $L$ on $H$, i.e. $\|L\|<\infty$. This acts as a linear system that maps a finite-norm input signal $f(t)$ to a finite-norm output signal

$$
g(t)=L[f(t)]
$$

If we consider the output value at any time location $t=t_{o}$, then the map $f(t) \mapsto g\left(t_{o}\right)=\phi(f)$ is a bounded linear functional on $H$. From the Riesz representation theorem, there is a unique signal $k_{t_{o}} \in H$ such that

$$
g\left(t_{o}\right)=\left.L[f(t)]\right|_{t=t_{o}}=\left\langle f, k_{t_{o}}\right\rangle=\int f(\tau) k_{t_{o}}{ }^{*}(\tau) d \tau, \quad \forall t_{o} \in \mathbb{R}
$$

Clearly, the function $k_{t_{o}}(t)$ is the complex conjugate of the system's impulse response map at time $t=t_{o}$, i.e. the system's output

$$
h_{t_{o}}(t) \triangleq L\left[\delta\left(t-t_{o}\right)\right]
$$

when the input is an impulse function $\delta\left(t-t_{o}\right)$ located at $t=t_{o}$. Note that the above function is a map that assigns a possibly different signal at each time instant $t_{o}$ because the system may be time-varying. 
However, if the linear system $L$ is also time-invariant, then there exists a unique function $h(t)=L[\delta(t)]$, called impulse response of the system, such that

$$
h_{t_{o}}(t)=h\left(t-t_{o}\right), \quad \forall t_{o} \in \mathbb{R}
$$

In this case, the Riesz representation theorem implies that the system's output signal $g$ is the continuous-time linear convolution of the input signal $f$ and the impulse response $h$ :

$$
g(t)=L[f(t)]=\int f(\tau) h(t-\tau) d \tau
$$

The above ideas easily carry over to linear systems processing discrete-time signals, i.e. bounded linear operators $L$ on the Hilbert space $H=\ell^{2}(\mathbb{Z}, \mathbb{C})$. If $f[n]$ is an input sequence and $g[n]=$ $L(f[n])$ the corresponding output, then for each $n=n_{o}$ there is a unique finite-energy sequence $h_{n_{o}}=L\left(\delta\left[n-n_{o}\right]\right)$, defined as the system's response for input impulse at location $n=n_{o}$, such that

$$
g[n]=L[f[n]]=\sum_{m} f[m] h_{n_{o}}[n-m]
$$

If the system is also time-invariant, there is a unique sequence $h[n]=L(\delta[n]$ called the impulse response, such that $h_{n_{o}}[n]=h\left[n-n_{o}\right]$ for all $n_{o}$, and hence the system's input-output relationship is given by a discrete-time linear convolution:

$$
g[n]=L[f[n]]=\sum_{m} f[m] h[n-m]
$$

Finally, the Riesz representation theorem and its consequences (e.g. the convolutional expression in case of shift-invariance) directly apply to bounded linear operators on spaces of multidimensional signals.

\subsubsection{Spectral Representation of Linear Operators on Finite-dimensional Vector Spaces}

The main idea here is to perform a geometrical analysis of a complex Hilbert space $H$ by decomposing it into a sum of simpler subspaces, e.g. those formed as ranges of projections, while a linear operator $L$ on $H$ is expressed as a corresponding superposition of simpler operations, e.g. projections on these subspaces. This also leads to a spectral analysis of the operator via its eigenvalues and eigenvectors. We shall outline from Naylor and Sell (1982) some main results in the case where the decomposition is done with a finite number of projections.

A collection $\left\{P_{1}, P_{2}, \ldots, P_{m}\right\}$ of continuous projections on $H$ is called a resolution of the identity if (i) they are orthogonal, (ii) $P_{i} P_{j}=O$ if $i \neq j$, and (iii) $I=P_{1}+\cdots P_{m}$. Without any loss of generality we shall henceforth assume that $P_{i} \neq O$ for all $i$. Then, the space can be decomposed as

$$
H=\operatorname{Ran}\left(P_{1}\right) \oplus \cdots \oplus \operatorname{Ran}\left(P_{m}\right)
$$

where $\operatorname{Ran}\left(P_{i}\right) \perp \operatorname{Ran}\left(P_{j}\right)$ if $i \neq j$. Let $\left\{\lambda_{1}, \ldots, \lambda_{m}\right\}$ be a set of distinct scalars and consider the linear operator

$$
L=\lambda_{1} P_{1}+\cdots+\lambda_{m} P_{m}
$$

Then, $L$ is continuous and normal. Further, it is self-adjoint iff all the $\lambda_{i}$ 's are real. Note that, $L$ agrees with $\lambda_{i} I$ on $\operatorname{Ran}\left(P_{i}\right)$. Thus, since any 'vector' $x \in H$ can be decomposed uniquely as

$$
x=x_{1}+\cdots+x_{m}, \quad x_{i} \in \operatorname{Ran}\left(P_{i}\right),
$$

the action of $L$ on $x$ can also be decomposed as

$$
L(x)=\lambda_{1} x_{1}+\cdots+\lambda_{m} x_{m}
$$


The $\left\{\lambda_{i}\right\}$ are called the eigenvalues of $L$ because they are the only scalar solutions of the equation

$$
L(x)=\lambda x
$$

For each $\lambda_{i}$ the corresponding eigenvector must lie in $\operatorname{Ran}\left(P_{i}\right)$. Actually, there exists an orthonormal basis of eigenvectors $\left\{e_{i}\right\}$ of $L$. By using it, we can express any point $x \in H$ (input to the operator) as

$$
x=\sum_{i=1}^{m}\left\langle x, e_{i}\right\rangle e_{i}
$$

and the corresponding output from the operator as

$$
L(x)=\sum_{i=1}^{m} \lambda_{i}\left\langle x, e_{i}\right\rangle e_{i}
$$

The norm of the operator can also be expressed via its eigenvalues:

$$
\|L\|=\max _{i}\left|\lambda_{i}\right|
$$

The above analysis was relatively easy because we started from synthesizing a linear operator as a finite linear combination of known orthogonal projections that can build up completely the whole space. A more difficult problem is to start from a linear operator and then find a set of such orthogonal projections. For further details the reader is referred to Naylor and Sell (1982).

\section{Lattice Spaces and Morphological Image Operators}

\subsection{Lattices: Synopsis}

Most of the material in Section 3.1 follows Birkhoff (1967), Heijmans and Ronse (1990), and Heijmans (1994).

A partially-ordered set, briefly poset $(\mathcal{P}, \leq)$, is a set $\mathcal{P}$ in which a binary relation $\leq$ is defined that is a partial ordering, i.e., satisfies the following three properties for all $X, Y, Z \in \mathcal{P}$ :

$$
\begin{array}{llr}
\text { P1. } & X \leq X \\
\text { P2. } & X \leq Y \text { and } Y \leq X \text { imply } X=Y & \text { (reflexive) } \\
\text { P3. } & X \leq Y \text { and } Y \leq Z \text { imply } X \leq Z & \text { (transitive) }
\end{array}
$$

If $\leq$ has the additional property that, for any two elements we have either $X \leq Y$ or $Y \leq X$, then $\mathcal{P}$ is called a totally-ordered set or chain.

To every partial ordering $\leq$ on $\mathcal{P}$ there corresponds a dual partial ordering $\leq$ defined by " $X \leq^{\prime} Y$ iff $X \geq Y$ ". Duality Principle: If $(\mathcal{P}, \leq)$ is a poset, then $\left(\mathcal{P}, \leq^{\prime}\right)$ is also a poset, called the dual poset.

Let $\mathcal{S}$ be a subset of a poset $(\mathcal{P}, \leq)$. An upper bound of $\mathcal{S}$ is an element $B \in \mathcal{P}$ such that $X \leq B$ for all $X \in \mathcal{S}$. The least upper bound of $\mathcal{S}$ is called its supremum and denoted by $\sup \mathcal{S}$ or $\bigvee \mathcal{S}$. By using the duality principle, we can also define the concepts of a lower bound and the greatest lower bound of $\mathcal{S}$; the latter is called its infimum and denoted by $\inf \mathcal{S}$ or $\wedge \mathcal{S}$. The supremum and infimum are unique if they exist. If the supremum (resp. infimum) of $\mathcal{S}$ belongs to $\mathcal{S}$, then it is called the the greatest element or maximum (resp. least element or minimum) of $\mathcal{S}$. If the poset $\mathcal{P}$ is not easily inferred from the context, the supremum and infimum of a subset $\mathcal{S}$ may be

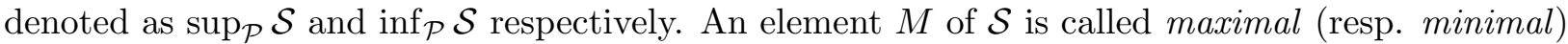
if there is no other element in $\mathcal{S}$ that is greater (resp. smaller) than $M$ with respect to $\leq$.

A lattice is a poset $(\mathcal{L}, \leq)$ any two of whose elements have a supremum, denoted by $X \vee Y$, and an infimum, denoted by $X \wedge Y$. We often denote the lattice structure by $(\mathcal{L}, \vee, \wedge)$. A lattice is $\mathcal{L}$ is complete if each of its subsets has a supremum and an infimum in $\mathcal{L}$. Any nonempty complete 
lattice is universally bounded because it contains its supremum $I=\bigvee \mathcal{L}$ and infimum $O=\bigwedge \mathcal{L}$ which are its greatest and least elements, respectively. Sometimes $O$ is called the 'null element' and $I$ is called the 'all element'. If $\mathcal{L}$ is not easily inferred from the context, we may denote its least and greatest elements by $\mathcal{L}_{O}$ and $\mathcal{L}_{I}$ respectively.

Duality in Lattices: In any lattice $\mathcal{L}$, by replacing the partial ordering $\leq$ with its dual $\leq^{\prime}$ and by interchanging the roles of the supremum and infinum, i.e., by considering the dual operations $\vee^{\prime}=\wedge$ and $\wedge^{\prime}=\vee$, we can form a new lattice $\left(\mathcal{L}, \vee^{\prime}, \wedge^{\prime}\right)$, called the dual lattice and often denoted just by $\mathcal{L}^{\prime}$. The duality principle dictates that to every definition, property and statement that applies to the lattice $\mathcal{L}$ there corresponds a dual one that applies to the dual lattice $\mathcal{L}^{\prime}$ by interchanging $\leq$ with $\leq^{\prime}$ and $\vee$ with $\wedge$.

A bijection between two lattices $\mathcal{L}$ and $\mathcal{M}$ is called an isomorphism (resp. dual isomorphism) if it preserves (resp. reverses) suprema and infima. If $\mathcal{L}=\mathcal{M}$, a (dual-)isomorphism on $\mathcal{L}$ is called (dual-)automorphism. A lattice is called self-dual if there is a dual automorphism on it; it can be shown that a lattice $\mathcal{L}$ is self-dual iff it is isomorphic with its dual lattice $\mathcal{L}^{\prime}$.

\subsubsection{Lattice Properties}

The lattice operations satisfy many properties, the most fundamental of which are summarized in Table 1. Conversely, a set $\mathcal{L}$ equipped with two binary operations $\vee$ and $\wedge$ that satisfy the first four pairs of properties, i.e., (L1,L1 $\left.{ }^{\prime}\right)-\left(\mathrm{L} 4, \mathrm{~L} 4^{\prime}\right)$ is lattice whose supremum is $\vee$, infimum is $\wedge$, and partial ordering $\leq$ is given by L5. Note that, properties $\left(\mathrm{L} 6, \mathrm{~L} 6^{\prime}\right)$ and $\left(\mathrm{L} 7, \mathrm{~L} 7^{\prime}\right)$ hold only if the lattice contains a least and a greatest element, respectively.

Table 1: Properties of Lattice Operations

\begin{tabular}{|c|c|c|}
\hline Sup-Semilattice & Inf-Semilattice & Description \\
\hline $\bar{X} X \vee X=X$ & $\begin{array}{ll}\mathrm{L}^{\prime} . & X \wedge X=X \\
\end{array}$ & Idempotence \\
\hline$X \vee Y=Y \vee X$ & $\mathrm{~L}^{\prime} . \quad X \wedge Y=Y \wedge X$ & Commutativity \\
\hline$X \vee(Y \vee Z)=(X \vee Y) \vee Z$ & L3 $^{\prime} . \quad X \wedge(Y \wedge Z)=(X \wedge Y) \wedge Z$ & Associativity \\
\hline$X \vee(X \wedge Y)=X$ & $X \wedge(X \vee Y)=X$ & Absorption \\
\hline$X \leq Y \Longleftrightarrow Y=X \vee Y$ & L5 $^{\prime} . \quad X \geq Y \Longleftrightarrow Y=X \wedge Y$ & Consistency \\
\hline$O \vee O \vee X=X$ & $\begin{array}{ll}\text { L6. } & I \wedge X=X \\
\end{array}$ & Identity \\
\hline L7. $\quad I \vee X=I$ & L7. $\quad O \wedge X=O$ & Absorbing Null \\
\hline
\end{tabular}

A lattice $\mathcal{L}$ is called distributive if

$$
A \wedge\left(\bigvee_{i \in J} X_{i}\right)=\bigvee_{i \in J}\left(A \wedge X_{i}\right) \text { and } A \vee\left(\bigwedge_{i \in J} X_{i}\right)=\bigwedge_{i \in J}\left(A \vee X_{i}\right)
$$

for any finite index set $J$ and for all $A, X_{i} \in \mathcal{L}$. If the above also holds for infinite index sets, then the lattice is called infinitely distributive.

A lattice $\mathcal{L}$ is called modular if $\forall X, Y, Z \in \mathcal{L}$

$$
X \leq Z \Longrightarrow X \vee(Y \wedge Z)=(X \vee Y) \wedge Z
$$

Obviously, a distributive lattice is modular, but the converse is not always true.

\subsubsection{Semilattices}

A lattice contains two weaker substructures, i.e., two semilattices. In general, a semilattice $(\mathcal{P}, \circ)$ is a set $\mathcal{P}$ equipped with a binary operation $\circ$ that is associative, commutative, and idempotent. Actually, any semilattice $(\mathcal{P}, \circ)$ is a poset in which the partial ordering is defined by $X \leq Y$ iff 
$Y=X \circ Y$ and any two elements possess a supremum ${ }^{6}$ defined by $X \vee Y=X \circ Y$; such a semilattice is called a sup-semilattice. Conversely, if $(\mathcal{P}, \leq)$ is a poset in which any two elements $X, Y$ have a supremum $X \circ Y$, then $\mathcal{P}$ is a semilattice with respect to the binary operation $\circ$. It now becomes evident that a lattice $(\mathcal{L}, \vee, \wedge)$ contains a sup-semilattice $(\mathcal{L}, \vee)$ that satisfies properties L1-L3 of Table 1, an inf-semilattice $(\mathcal{L}, \wedge)$ that satisfies properties L1'-L3', and the two binary operations of supremum and infimum are related via properties L4,L4 ${ }^{\prime}$ that make them dual to each other.

\subsubsection{Sublattices and Sup/Inf-closed subsets}

Let $(\mathcal{L}, \vee, \wedge)$ be a lattice with partial ordering $\leq$ and consider a nonempty subset $\mathcal{S}$ of $\mathcal{L}$. Under the same partial ordering, $(\mathcal{S}, \leq)$ is a poset. If $\mathcal{S}$ is also closed under finite suprema and infima that are induced by the partial ordering of $\mathcal{L}$, then $\mathcal{S}$ is a called an underlattice of $\mathcal{L}$. If $\mathcal{S}$ is an underlattice whose supremum and infimum induced by $\leq$ are the same operations $(\vee, \wedge)$ as those of $\mathcal{L}$, then $\mathcal{S}$ is called a sublattice of $\mathcal{L}$. A sublattice is always an underlattice, but the converse is not always true. Underlattices and sublattices are complete if they remain closed under infinite suprema and infima.

A subset $\mathcal{S}$ of a complete lattice $\mathcal{L}$ is called sup-closed (resp. inf-closed) if any subcollection of its elements has its $\mathcal{L}$ supremum (resp. infimum) in $\mathcal{S}$. The extreme case where this subcollection is empty implies that $O \in \mathcal{S}$ if $\mathcal{S}$ is a sup-closed (resp. $I \in \mathcal{S}$ if $\mathcal{S}$ is inf-closed). It turns out that in a complete lattice, any sup-closed or inf-closed subset is a complete underlattice; if a subset is both inf- and sup-closed, then it is a complete sublattice.

Example 1 (Lattices and Sublattices):

(a) Any chain is a lattice under the chain order, because the supremum and infimum of any pair of elements exist and equal their maximum and minimum, respectively. Thus, the chain $(\mathbb{R}, \leq)$ of real numbers equipped with the natural order $\leq$ is a lattice, but it is not complete. However, if we consider the lattice $(\overline{\mathbb{R}}, \vee, \wedge)$, where

$$
\overline{\mathbb{R}}=\mathbb{R} \cup\{-\infty,+\infty\}
$$

is the set of extended real numbers, then $\overline{\mathbb{R}}$ is a complete lattice and all its subsets have a supremum and infimum. For example, $\sup \mathbb{R}=+\infty$ and $\inf \mathbb{R}=-\infty$. $\overline{\mathbb{R}}$, as well as any other complete chain, is completely distributive. ${ }^{7}$ The set $\overline{\mathbb{Z}}=\mathbb{Z} \cup\{-\infty,+\infty\}$ of extended integers is a complete sublattice of $\overline{\mathbb{R}}$. In contrast, the chain $\overline{\mathbb{Q}}=\mathbb{Q} \cup\{-\infty,+\infty\}$ of extended rationals is only an incomplete sublattice of $\overline{\mathbb{R}}$.

(b) The power set $\mathcal{P}(E)$ of an arbitrary set $E$ equipped with the partial order of set inclusion is the archetypal poset. It is also a completely distributive lattice under the supremum and infimum induced by set inclusion which are the set union and intersection, respectively. Any collection of subsets of $E$ that is closed under finite unions and intersections is called a ring of sets. Any such set ring is a sublattice of the power set $\mathcal{P}(E)$. If $E$ is a topological space, e.g. the Euclidean space $\mathbb{R}^{m}$, let $\mathcal{F}(E)$ be the collection of its closed subsets. Then, $\mathcal{F}(E)$ is an inf-closed subset and hence a complete underlattice of $\mathcal{P}(E)$; further, $\mathcal{F}(E)$ is an incomplete sublattice of $\mathcal{P}(E)$.

(c) If $V$ is a linear space, then $(\mathcal{P}(V), \cup, \cap)$ is a complete lattice, whose partial ordering, supremum and infimum are the set inclusion, union and intersection, respectively. The greatest and least elements of $\mathcal{P}(V)$ are $V$ and $\varnothing$, respectively. Let now $\mathcal{S}$ be the collection of linear subspaces of $V$. Then, $\mathcal{S}$ is inf-closed but not sup-closed. Actually, $\mathcal{S}$ becomes a complete underlattice of $\mathcal{P}(V)$ equipped with set intersection as its infimum and with (Minkowski) sum as its supremum. The greatest and least elements of $\mathcal{S}$ are $V$ and $\{\mathbf{0}\}$, respectively.

\footnotetext{
${ }^{6}$ If in the semilattice $(\mathcal{P}, \circ)$ we consider a different partial ordering $X \leq^{\prime} Y \Longleftrightarrow Y=X \circ Y$ where $X \circ Y$ is interpreted as the infimum $X \wedge Y$, then $(\mathcal{P}, \circ)$ becomes an inf-semilattice and vice-versa. Obviously, $\left(\mathcal{P}, \leq^{\prime}\right)$ is the dual poset of $(\mathcal{P}, \leq)$.

${ }^{7}$ The complete distributivity is an even stronger property than the infinite distributivity of (28) with infinite index set; it is explained in Birkhoff (1967) and Heijmans (1994).
} 


\subsubsection{Sup-generators, Atoms}

A subset $\mathcal{A}$ of a complete lattice $\mathcal{L}$ is called sup-generating (resp. inf-generating) if each lattice element can be expressed as a supremum (resp. infimum) of members of $\mathcal{A}$. Such generators can be found if the lattice has atom-like elements. Specifically, an element $A \in \mathcal{L}$ is called an atom if it is a minimal element of $\mathcal{L} \backslash\{O\}$, i.e. if $A \in \mathcal{L} \backslash\{O\}$ and, for each $X \in \mathcal{L}, X \leq A$ implies $X=O$ or $X=A$. Respectively, a dual atom is called any maximal element of $\mathcal{L} \backslash\{I\}$. For example, the lattice of any power set $\mathcal{P}(E)$ has as atoms all the singletons.

A lattice is called atomic (resp. dual atomic) if the class of its atoms (resp. dual atoms) is sup-generating (resp. inf-generating). For example, the lattice $\mathcal{P}(E)$ is atomic since any subset of $E$ is the union of the singletons of the points it contains.

Atoms may not exist in a lattice. For example, $\overline{\mathbb{R}}$ is an atomless chain. Further, let us henceforth denote collections of functions with common domain and range by

$$
\operatorname{Fun}(X, Y) \triangleq \text { set of all functions } f: X \rightarrow Y
$$

Then, the lattice $\operatorname{Fun}(E, \overline{\mathbb{R}})$ partially ordered by ' $f \leq g \Longleftrightarrow f(x) \leq g(x) \forall x$ ' is also atomless. A weaker concept is the semi-atom: a nonzero element $A \in \mathcal{L}$ is called a semi-atom if $A \leq X \vee Y$ implies $A \leq X$ or $A \leq Y$. A lattice is called semi-atomic if the class of its semi-atoms is supgenerating. For example, all the elements of any chain are semi-atoms and any chain is semi-atomic. Another example is the lattice $\mathcal{L}=\operatorname{Fun}(E, \overline{\mathbb{R}})$ where we consider the impulse functions

$$
q_{h, v}(x)= \begin{cases}v, & x=h \\ -\infty, & x \neq h\end{cases}
$$

Then these impulses are semi-atoms and the lattice is semi-atomic since any function $f$ in the lattice can be written as supremum of some semi-atoms:

$$
f(x)=\bigvee_{h \in E} q_{h, f(h)}(x)
$$

In lattices with semi-atoms, we can also define dual semi-atoms (as the atoms of the dual lattice), which, if they form an inf-generating class, create a dual semi-atomic lattice.

If they exist, the (semi-)atoms of a lattice are preserved by a lattice isomorphism, whereas they are mapped to dual (semi-)atoms by a dual isomorphism.

\subsubsection{Boolean Lattices}

In a lattice $\mathcal{L}$ with universal bounds $O$ and $I$, an element $X \in \mathcal{L}$ is said to have a complement $X^{*} \in \mathcal{L}$ if $X \vee X^{*}=I$ and $X \wedge X^{*}=O$. For example, if $\mathcal{L}$ is a power set $\mathcal{P}(E)$, then $X^{*}$ is the well-known set complement $X^{c}=E \backslash X$. If all the elements of a general lattice $\mathcal{L}$ have complements, then $\mathcal{L}$ is called complemented. A Boolean lattice is defined as any lattice that is complemented and distributive. In any Boolean lattice $\mathcal{B}$, the complement of each element is unique and involutive: $\left(X^{*}\right)^{*}=X$. Actually, the mapping $X \mapsto X^{*}=\nu(X)$ is a negation (i.e. an involutive dual automorphism) on $\mathcal{B}$. Thus, any Boolean lattice is self-dual.

For each $X, Y \in \mathcal{B}$ the following additional properties hold:

$$
\begin{array}{lll}
X \vee X^{*}=I & \text { and } & X \vee X^{*}=O \\
(X \vee Y)^{*}=X^{*} \wedge Y^{*} & \text { and } & (X \wedge Y)^{*}=X^{*} \vee Y^{*}
\end{array}
$$

The set $\mathcal{B}$, equipped with the two binary operations of supremum and infimum and with the unary operation of the complement, becomes an algebra. When so considered, a Boolean lattice is called a Boolean algebra.

A collection $\mathcal{S}$ of subsets of some arbitrary universal set $E$ is called a field of sets if it is closed under finite unions, intersections and complementation. This structure is isomorphic to Boolean lattices, as explained by the following representation theorem, which can be found in Halmos (1963). 
Theorem 8 (a) Any Boolean lattice is isomorphic to a field of sets. (b) A Boolean lattice is complete and atomic iff it is isomorphic to the power set $\mathcal{P}(E)$ of some set $E$.

A finite Boolean algebra is obviously a complete and atomic lattice. Hence, it is isomorphic to the power set of the set of its atoms. Thus, the cardinality of a finite Boolean algebra must be of the form $2^{n}$, where $n \geq 1$ is the number of atoms.

Example 2 (Boolean algebras and Boolean Functions):

(a) The simplest Boolean algebra is the set $B=\{0,1\}$ equipped with the operations

$$
x \vee y=x \overline{+} y, \quad x \wedge y=x \cdot y, \quad x^{*}=\bar{x}=1-x
$$

where $\bar{\mp}$ denotes addition modulo 2 , and $\overline{(\cdot)}$ denotes Boolean complement.

(b) Consider the set $B V_{n}=\{0,1\}^{n}=\left\{\mathbf{v}=\left(v_{1}, \ldots, v_{n}\right): v_{i} \in\{0,1\}\right\}$ of $n$-dimensional Boolean vectors equipped with the operations

$$
\begin{aligned}
\mathbf{v} \vee \mathbf{u} & =\mathbf{v} \overline{+} \mathbf{u}=\left(v_{1} \bar{\mp} u_{1}, \ldots, v_{n} \bar{\mp} u_{n}\right) \\
\mathbf{v} \wedge \mathbf{u} & =\mathbf{v} \cdot \mathbf{u}=\left(v_{1} u_{1}, \ldots, v_{n} u_{n}\right) \\
\mathbf{v}^{*} & =\overline{\mathbf{v}}=\left(\overline{v_{1}}, \ldots, \bar{v}_{n}\right)
\end{aligned}
$$

The partial ordering of $B V_{n}$ is

$$
\mathbf{v} \leq \mathbf{u} \Longleftrightarrow v_{i} \leq u_{i} \forall i=1, \ldots, n
$$

Then, $B V_{n}$ is a Boolean algebra with $2^{n}$ elements. Its atoms are the $n$ vectors that have exactly one component equal to 1 .

(c) Consider the set $B F_{n}=\operatorname{Fun}\left(\{0,1\}^{n},\{0,1\}\right)$ of Boolean functions with $n$ variables. Given any such Boolean functions $b, b_{1}, b_{2}$ we can define the following two binary and one unary operations:

$$
\begin{aligned}
\left(b_{1} \vee b_{2}\right)(\mathbf{v}) & =\left(b_{1} \overline{+} b_{2}\right)(\mathbf{v})=b_{1}(\mathbf{v}) \mp b_{2}(\mathbf{v}) \\
\left(b_{1} \wedge b_{2}\right)(\mathbf{v}) & =\left(b_{1} \cdot b_{2}\right)(\mathbf{v})=b_{1}(\mathbf{v}) \cdot b_{2}(\mathbf{v}) \\
b^{*}(\mathbf{v}) & =\bar{b}(\mathbf{v})=\overline{b(\overline{\mathbf{v}})}
\end{aligned}
$$

Under these operations the set $B F_{n}$ becomes a Boolean algebra of $2^{2^{n}}$ elements. The corresponding partial ordering is

$$
b_{1} \leq b_{2} \Longleftrightarrow b_{1}(\mathbf{v}) \leq b_{2}(\mathbf{v}) \forall \mathbf{v} \in\{0,1\}^{n}
$$

\subsubsection{Function Lattices}

An easy and frequent way of creating new lattices out of existing lattices is by forming mappings from an arbitrary nonempty set $E$ to an existing lattice $(\mathcal{L}, \vee, \wedge)$. Let $\mathcal{L}^{E}=\operatorname{Fun}(E, \mathcal{L})$ be the set of all functions $f: E \rightarrow \mathcal{L}$. If $\leq$ is the partial ordering of the lattice $\mathcal{L}$, we can equip the function space $\mathcal{L}^{E}$ with the following pointwise partial ordering:

$$
f \leq g \Longleftrightarrow f(x) \leq g(x) \forall x \in E
$$

This induces the following pointwise supremum $\bigvee_{i} f_{i}$, and pointwise infimum $\bigwedge_{i} f_{i}$ on the function space $\mathcal{L}^{E}$ :

$$
\begin{aligned}
& \left(\bigvee_{i \in J} f_{i}\right)(x)=\bigvee_{i \in J} f_{i}(x), \quad x \in E \\
& \left(\bigwedge_{i \in J} f_{i}\right)(x)=\bigwedge_{i \in J} f_{i}(x), \quad x \in E
\end{aligned}
$$


where $J$ is an abritrary index set. Thus, $\left(\mathcal{L}^{E}, \vee, \wedge\right)$ is lattice called a power lattice or function lattice. These lattices are very useful for modeling lattice spaces of finite- or infinite-support images and unifying their properties. For instance, if $\mathcal{L}$ has any of the properties of being complete, or distributive, or infinitely distributive, or Boolean, then the function lattice $\mathcal{L}^{E}$ retains these properties. Preservation of the Boolean property was already encountered in Example 2.

Two useful cases of function lattices are the following: (1) Vector lattices: If $E=\{1,2, \ldots, n\}$, then $\mathcal{L}^{E}$ becomes the set of all $n$-dimensional vectors $\left(x_{1}, \ldots, x_{n}\right)$ with elements from $\mathcal{L}$. (2) Signal lattices: If $E=\mathbb{R}^{m}$ or $E=\mathbb{Z}^{m}$, then $\mathcal{L}^{E}$ becomes the set of all $m$-dimensional signals (with continuous or discrete domain) with values from $\mathcal{L}$. The special case of the lattice $\{0,1\}^{E}$ of binary-valued signals is isomorphic to the set lattice $\mathcal{P}(E)$.

\subsection{Image Lattices}

We shall deal mainly with function lattices. The underlying set of these lattices is the collection $\mathcal{S}=\operatorname{Fun}(E, \mathcal{C})$ of all image (or image-related) functions $f: E \rightarrow \mathcal{C}$ whose domain is an arbitrary nonempty set $E$ and their range is the value set $\mathcal{C}$ which is a complete lattice. Of main importance is the case $E=\mathbb{R}^{m}$ or $E=\mathbb{Z}^{m}, m=1,2,3$, where $\mathcal{S}$ becomes the set of all spatio-temporal image signals defined on the continuous Euclidean space or its discretized version on a regular grid and taking values in $\mathcal{C}$. We denote the multi-dimensional image signal domain in both of these cases by $\mathbb{E}^{m}$ where $\mathbb{E}=\mathbb{R}$ or $\mathbb{Z}$. But $E$ could also be a finite index set for matrix-based image processing or the set of vertices of a graph in cases of images defined on a graph. Let the partial order, supremum and infimum of $\mathcal{C}$ be $\leq, \vee$, and $\wedge$, respectively. Then, the complete lattice structure of $\mathcal{C}$ is also inherited by the function $\operatorname{space} \operatorname{Fun}(E, \mathcal{C})$ by extending the partial order, supremum and infimum among elements in $\mathcal{C}$ to functions in $\mathcal{S}$ pointwise, as in (38), (39) and (40). The function lattice $\operatorname{Fun}(E, \mathcal{C})$ inherits many of the extra properties that the value lattice $\mathcal{C}$ may have, such as its distributivity type or Boolean nature.

Example 3 (Lattices of Shapes and Images):

The main examples of complete and infinitely distributive lattices used in image analysis are:

(a) The space $\mathcal{P}\left(\mathbb{E}^{m}\right)$ of all $m$-dimensional shapes represented by subsets of the $m$-dimensional continuous plane $\mathbb{E}^{m}=\mathbb{R}^{m}$ or the discrete plane $\mathbb{E}^{m}=\mathbb{Z}^{m}$ equipped with set inclusion $\subseteq$ as the partial ordering. The induced supremum and infimum operations are the set union and intersection, respectively. This set lattice is isomorphic to the function lattice of all $m$-dimensional binary images $f: \mathbb{E}^{m} \rightarrow\{0,1\}$. Both are complete, infinitely distributive, atomic Boolean lattices.

(b) The space of all graylevel image signals $f: \mathbb{E}^{m} \rightarrow \overline{\mathbb{R}}$ where the partial ordering $\leq$, supremum $\bigvee$ and infimum $\bigwedge$ are defined by extending pointwise the corresponding notions from the value lattice $\overline{\mathbb{R}}$ to functions. Intensity images usually have as range $[0, \infty]$, or $[0,1]$ if they are normalized; both ranges are complete sublattices of $\overline{\mathbb{R}}$. Density images, i.e. logarithms of intensity images have range $\overline{\mathbb{R}}$.

(c) Spaces of vector-valued images, e.g. color images or moving images, can be modeled as complete lattices by using several different partial orderings, as explained in Goutsias et al. (1995).

Example 4 (Lattices of Semi-continuous and Lipschitz Image Functions):

(a) Within the lattice $\operatorname{Fun}\left(\mathbb{R}^{m}, \overline{\mathbb{R}}\right)$ of all $m$-dimensional extended real-valued functions defined on $\mathbb{R}^{m}$, let us consider the subcollections $\operatorname{Fun}_{\text {usc }}\left(\mathbb{R}^{m}, \overline{\mathbb{R}}\right)$ and $\operatorname{Fun}_{l s c}\left(\mathbb{R}^{m}, \overline{\mathbb{R}}\right)$, respectively, of all upper semi-continuous (u.s.c.) and lower semi-continuous (l.s.c. $)^{8}$ functions. As explained in Heijmans and Maragos (1997), the pointwise infimum (resp. supremum) of any collection of u.s.c. (resp. l.s.c.) functions is still an u.s.c. (resp. l.s.c.) function. Let $\beta_{u}(f)$ denote the upper closed hull of $f$ which is defined as the infimum of all u.s.c. functions that lie above $f$. Dually, let $\alpha_{l}(f)$ denote

\footnotetext{
${ }^{8} \mathrm{~A}$ function $f: \mathbb{R}^{m} \rightarrow \overline{\mathbb{R}}$ is upper semi-continuous (u.s.c.) [resp. lower semi-continuous (l.s.c.)] iff, for each $\mathbf{x} \in \mathbb{R}^{m}$ and $t \in \overline{\mathbb{R}}, f(\mathbf{x})<t$ (resp. $f(\mathbf{x})>t$ ) implies that $f(\mathbf{y})<t$ (resp. $f(\mathbf{y})>t$ ) for all $\mathbf{y}$ in some neighborhood of $\mathbf{x}$. Equivalently, $f$ is u.s.c. (resp. l.s.c.) iff all its level sets are closed (resp. open) subsets of $\mathbb{R}^{m}$. A function is continuous iff it is both u.s.c. and l.s.c.
} 
the lower closed hull of $f$ which is defined as the supremum of all l.s.c. functions below $f$. It can be shown that

$$
\alpha_{l}(f)(\mathbf{x})=\liminf _{y \rightarrow x} f(\mathbf{y}), \quad \beta_{u}(f)(\mathbf{x})=\limsup _{y \rightarrow x} f(\mathbf{y})
$$

As explained by the next result, the two above collections of semi-continuous functions are complete underlattices of $\operatorname{Fun}\left(\mathbb{R}^{m}, \overline{\mathbb{R}}\right)$. Further, their intersection, i.e. the collection $\operatorname{Fun}\left(\overline{\mathbb{R}}^{m}, \mathbb{R}\right)$ of continuous functions is a complete sublattice.

Proposition 2 (Heijmans and Maragos, 1997).

(a) The set $\operatorname{Fun}_{\text {usc }}\left(\mathbb{R}^{m}, \overline{\mathbb{R}}\right)$ is a complete lattice under the pointwise ordering with the pointwise infimum $\bigwedge_{i} f_{i}$, and with supremum given by $\beta_{u}\left(\bigvee_{i} f_{i}\right)$.

(b) The set $\operatorname{Fun}_{l s c}\left(\mathbb{R}^{m}, \overline{\mathbb{R}}\right)$ is a complete lattice under the pointwise ordering with the pointwise supremum $\bigvee_{i} f_{i}$, and with infimum given by $\alpha_{l}\left(\bigwedge_{i} f_{i}\right)$.

(b) A real-valued function $f$ on $\mathbb{R}^{m}$ is called globally Lipschitz continuous (or just Lipschitz) if there exists a constant $K$ such that for all $\mathbf{x}, \mathbf{y}$

$$
|f(\mathbf{x})-f(\mathbf{y})| \leq K\|\mathbf{x}-\mathbf{y}\|
$$

The infimum of such constants $K$ is called its Lipschitz coefficient and denoted by $\operatorname{Lip}(f)$. By restricting for each point the domain on which the Lipschitz condition holds to an open neighborhood around that point, we can also define locally Lipschitz functions. Any Lipschitz function is uniformly continuous. Given that Lipschitz functions are differentiable almost everywhere and the magnitude of their derivatives is bounded by their constant $K$, they are the best choice (in terms of degree of regularity) between continuous and differentiable functions. As observed in Serra (1988, ch.12), the pointwise infimum and supremum of Lipschitz functions are still Lipschitz. Thus the collection $\operatorname{Fun}_{l i p}\left(\mathbb{R}^{m}, \mathbb{R}\right)$ of Lipschitz functions is a complete sublattice of the lattice of continuous functions. Another interesting property of Lipschitz functions is that they remain Lipschitz after their Minkowski dilation and erosion by another function, as shown in van den Boomgaard and Smeulders (1994).

\subsection{Image and Signal Operators on Lattices}

Let $\mathcal{L}$ be a complete lattice and let $\mathcal{O}(\mathcal{L})$ be the set of all operators on $\mathcal{L}$, i.e., mappings from $\mathcal{L}$ to itself. This is an example of a function lattice where the domain and value set are both equal to $\mathcal{L}$. Given two such operators $\psi$ and $\phi$ we define below a partial ordering $\leq$ between them, their supremum $(\psi \vee \phi)$ and infimum $(\psi \wedge \phi)$ in a pointwise way; further, we define their composition as an operator product $\psi \phi$ : for $X \in \mathcal{L}$

$$
\begin{gathered}
\phi \leq \psi \Longleftrightarrow \phi(X) \leq \psi(X) \forall X \\
(\psi \vee \phi)(X) \triangleq \psi(X) \vee \phi(X) \\
(\psi \wedge \phi)(X) \triangleq \psi(X) \wedge \phi(X) \\
\psi \phi(X) \triangleq \psi(\phi(X))
\end{gathered}
$$

Equipped with the above ordering, supremum and infimum, the set $\mathcal{O}(\mathcal{L})$ becomes a complete lattice which inherits many of the properties of $\mathcal{L}$.

Next follow the definitions of the identity and two constant operators as well as some general definitions for lattice operators $\psi$ that are related to their ordering or self-compositions:

$$
\begin{aligned}
\text { identity : } & \mathbf{i d}(X)=X \quad \forall X \in \mathcal{L} \\
\text { constant operators: } & \mathbf{O}(X)=\mathcal{L}_{O}, \mathbf{I}(X)=\mathcal{L}_{I}, \forall X \in \mathcal{L} \\
\text { extensive : } & \mathbf{i d} \leq \psi \\
\text { antiextensive : } & \psi \leq \mathbf{i d} \\
\text { idempotent : } & \psi \psi=\psi \\
\text { involution : } & \psi \psi=\mathbf{i d}
\end{aligned}
$$




\subsection{Monotone Lattice Operators}

Of great interest are the monotone operators. They come in three basic kinds according to which of the following three lattice structures they preserve (or map to its dual): (i) partial ordering, (ii) supremum, (iii) infimum.

A lattice operator $\psi$ is called increasing or isotone if it is order-preserving, i.e., $\forall X, Y$,

$$
\text { increasing : } \quad X \leq Y \Longrightarrow \psi(X) \leq \psi(Y)
$$

A lattice operator $\psi$ is called decreasing or antitone if it is order-inverting, i.e., $\forall X, Y$,

$$
\text { decreasing : } \quad X \leq Y \Longrightarrow \psi(X) \geq \psi(Y)
$$

It can be shown that an equivalent condition for an operator $\psi$ to be increasing is the following:

$$
\psi \text { is increasing } \Longleftrightarrow \psi(X \wedge Y) \leq \psi(X) \wedge \psi(Y) \Longleftrightarrow \psi(X) \vee \psi(Y) \leq \psi(X \vee Y)
$$

If the lattice is complete, the supremum and infimum in (42) can be taken on infinite collections.

\subsubsection{Increasing Operators}

Examples of increasing operators are the homomorphisms. A sup-homomorphism is any operator that preserves suprema over a finite collection, whereas an inf-homomorphism preserves infima. An operator $\phi: \mathcal{L} \rightarrow \mathcal{M}$ between two lattices $\mathcal{L}$ and $\mathcal{M}$ that combines both properties is called a lattice homomorphism:

$$
\phi\left(\bigvee_{i} X_{i}\right)=\bigvee_{i} \phi\left(X_{i}\right), \quad \phi\left(\bigwedge_{i} X_{i}\right)=\bigwedge_{i} \phi\left(X_{i}\right)
$$

If the lattice homomorphism is a bijection, then it is called a lattice isomorphism, or a lattice automorphism if $\mathcal{L}=\mathcal{M}$. It can be shown that an operator $\theta$ is a lattice isomorphism if it is a bijection and both $\theta$ and its inverse $\theta^{-1}$ are increasing:

$$
X \leq Y \Longleftrightarrow \theta(X) \leq \theta(Y)
$$

Four important types of increasing operators, which are fundamental for unifying lattice-based image analysis, are the following:

$$
\begin{array}{rll}
\delta \text { is dilation } & \text { iff } & \delta\left(\bigvee_{i \in J} X_{i}\right)=\bigvee_{i \in J} \delta\left(X_{i}\right) \\
\varepsilon \text { is erosion } & \text { iff } & \varepsilon\left(\bigwedge_{i \in J} X_{i}\right)=\bigwedge_{i \in J} \varepsilon\left(X_{i}\right) \\
\alpha \text { is opening } & \text { iff } & \alpha \text { is increasing, idempotent \& anti-extensive } \\
\beta \text { is closing } & \text { iff } & \beta \text { is increasing, idempotent \& extensive }
\end{array}
$$

The first two definitions require arbitrary (possibly infinite) collections $\left\{X_{i}: i \in J\right\}$ of lattice elements; hence, the dilation and erosion operators need complete lattices. The special case of an empty input collection equips each dilation and erosion with the following necessary properties:

$$
\delta(O)=O, \quad \varepsilon(I)=I
$$

The above definitions of lattice operators can be seen as generalizations of the corresponding translation-invariant Minkowski-type morphological image operators, whose definitions are given below.

Example 5 (Translation-Invariant Set Operators based on Minkowski set addition and subtraction):

(a) Automorphisms: On the image domain $\mathbb{E}^{m}$, consider the translations of sets $X \subseteq \mathbb{E}^{m}$ by vectors $\mathbf{z} \in \mathbb{E}^{m}:$

$$
X \mapsto X_{+\mathbf{z}} \triangleq\{\mathbf{x}+\mathbf{z}: \mathbf{x} \in X\}
$$


the reflection of a set:

$$
X \mapsto X^{s} \triangleq\{-\mathbf{x}: \mathbf{x} \in X\}
$$

and the uniform scaling of a set:

$$
X \mapsto r X \triangleq\{r \mathbf{x}: \mathbf{x} \in X\}, \quad r \in \mathbb{R} \backslash\{0\} .
$$

These three operators are automorphisms; hence they are both dilations and erosions.

(b) Some well-known (and familiar to image practitioners) special cases of the four above types of lattice operators are the following translation-invariant set operators on $\mathbb{E}^{m}$ :

$$
\begin{aligned}
& \delta_{B}(X)=X \oplus B \triangleq\left\{\mathbf{z}:\left(B^{s}\right)_{+\mathbf{z}} \cap X \neq \varnothing\right\}=\bigcup_{\mathbf{b} \in B} X_{+\mathbf{b}} \\
& \varepsilon_{B}(X)=X \ominus B \triangleq\left\{\mathbf{z}: B_{+\mathbf{z}} \subseteq X\right\}=\bigcap_{\mathbf{b} \in B} X_{-\mathbf{b}} \\
& \alpha_{B}(X)=X \circ B \triangleq(X \ominus B) \oplus B \\
& \beta_{B}(X)=X \bullet B \triangleq(X \oplus B) \ominus B
\end{aligned}
$$

In image processing applications, the set $B$ is called a structuring element and usually has a simple shape and small size. We shall refer to these translation-invariant operators as Minkowski operators, although historically the set dilation (a.k.a. Minkowski set addition) was developed by Minkowski (1903), whereas the set erosion (a.k.a. Minkowski set subtraction) and opening were developed by Hadwiger (1957). Minkowski-type operators were also developed for graylevel image analysis either as local min/max operations and their compositions in Meyer (1978), Nakagawa and Rosenfeld (1978), Goetcherian (1980), Serra (1982), or as max-plus convolutions in Sternberg (1980, 1986).

(c) Hit-Miss: The most famous non-monotone operator in morphological image analysis is the hit-miss transformation (Serra, 1982) of sets $X$ by a pair of disjoint structuring elements $(A, B)$ :

$$
X \otimes(A, B) \triangleq\left\{\mathbf{z}: A_{+\mathbf{z}} \subseteq X \text { and } B_{+\mathbf{z}} \subseteq X^{c}\right\}
$$

Despite its non-monotonicity, it is very useful for binary feature detection and extraction.

\subsubsection{Decreasing Operators}

Examples of decreasing operators are the dual homomorphisms, which interchange suprema with infima. These are the following.

$$
\begin{array}{lll}
\delta^{a} \text { is anti-dilation } & \text { iff } & \delta^{a}\left(\bigvee_{i \in J} X_{i}\right)=\bigwedge_{i \in J} \delta^{a}\left(X_{i}\right) \\
\varepsilon^{a} \text { is anti-erosion } & \text { iff } & \varepsilon^{a}\left(\bigwedge_{i \in J} X_{i}\right)=\bigvee_{i \in J} \varepsilon^{a}\left(X_{i}\right)
\end{array}
$$

where $J$ is an arbitrary (possibly infinite) index set.

Example 6 (Thresholding Operators):

Consider extended real-valued graylevel images $f: E \rightarrow \overline{\mathbb{R}}$ defined on an arbitrary set $E$ and the following operators mapping graylevel images to binary images represented by subsets of $E$ :

$$
\begin{aligned}
\varepsilon_{t}(f) & =\{x \in E: f(x) \geq t\} \\
\delta_{t}(f) & =\{x \in E: f(x) \leq t\}^{c} \\
\varepsilon_{t}^{a}(f) & =\{x \in E: f(x) \geq t\}^{c} \\
\delta_{t}^{a}(f) & =\{x \in E: f(x) \leq t\}
\end{aligned}
$$

Then, for any threshold level $t \in \overline{\mathbb{R}}, \varepsilon_{t}, \delta_{t}, \varepsilon_{t}^{a}$ and $\delta_{t}^{a}$ are, respectively, an erosion, dilation, antierosion, and anti-dilation (Banon and Barrera, 1993).

A lattice dual-isomorphism (or anti-isomorphism) is a bijection $\eta: \mathcal{L} \rightarrow \mathcal{M}$ between two lattices that interchanges suprema with infima; when $\mathcal{L}=\mathcal{M}, \eta$ is called a dual-automorphism. It can be 
shown that an operator $\eta$ is a lattice dual isomorphism if it is a bijection and both $\eta$ and its inverse $\eta^{-1}$ are decreasing:

$$
X \leq Y \Longleftrightarrow \eta(X) \geq \eta(Y)
$$

A negation $\nu$ is a dual automorphism that is also involutive, i.e., $\nu^{2}=\mathbf{i d}$; we often write $X^{*}=\nu(X)$ for the negative of a lattice element. Given an operator $\psi$ in a lattice equipped with a negation, its corresponding negative (also called dual) operator is defined as $\psi^{*}=\nu \psi \nu$; i.e.,

$$
\psi^{*}(X) \triangleq\left[\psi\left(X^{*}\right)\right]^{*}
$$

For example, the most well-known negation on the set lattice $\mathcal{P}(E)$ is the complementation $\nu(X)=$ $X^{c}=E \backslash X$, whereas on the function lattice $\operatorname{Fun}\left(\mathbb{E}^{m}, \overline{\mathbb{R}}\right)$ the most well-known negation is $\nu(f)=-f$.

The above definitions of increasing and decreasing operators allow broad classes of signal operators (monotone or not) to be grouped as parallel or sequential combinations of lattice dilations, or erosions, or openings, or closings, possibly composed with negations, and their common properties to be studied under the unifying lattice framework. Next we shall see several examples and applications of the above general definitions of lattice operators.

We conclude this section with some results on the complete lattices formed by the classes of increasing and decreasing operators as well as by the classes of dilations, erosions, anti-dilations and anti-erosions.

Proposition 3 (Heijmans and Ronse, 1990; Banon and Barrera, 1993).

Let $\mathcal{L}$ be a complete lattice. Then:

(a) The class $\mathcal{O}^{+}(\mathcal{L})$ of increasing operators on $\mathcal{L}$ is a complete sublattice of $\mathcal{O}(\mathcal{L})$.

(b) The class $\mathcal{O}^{-}(\mathcal{L})$ of decreasing operators on $\mathcal{L}$ is a complete sublattice of $\mathcal{O}(\mathcal{L})$.

(c) The class of dilations on $\mathcal{L}$ is a sup-closed subset and hence a complete underlattice of $\mathcal{O}^{+}(\mathcal{L})$.

(d) The class of erosions on $\mathcal{L}$ is an inf-closed subset and hence a complete underlattice of $\mathcal{O}^{+}(\mathcal{L})$.

(e) The class of anti-dilations on $\mathcal{L}$ is an inf-closed subset and hence a complete underlattice of $\mathcal{O}^{-}(\mathcal{L})$.

(f) The class of anti-erosions on $\mathcal{L}$ is a sup-closed subset and hence a complete underlattice of $\mathcal{O}^{-}(\mathcal{L})$.

Both sublattices $\mathcal{O}^{+}$and $\mathcal{O}^{-}$contain the greatest operator $(\mathbf{I})$ and least operator $(\mathbf{O})$ of $\mathcal{O}$. Further, the identity operator belongs to $\mathcal{O}^{+}$. In the lattice of dilations, the least element is $\mathbf{O}$ whereas the greatest element is a dilation that maps $O$ to itself and every input $X \neq O$ to $I$. Dually, in the lattice of erosions, the greatest element is $\mathbf{I}$ whereas the least element is an erosion that maps $I$ to itself and every input $X \neq I$ to $O$.

\subsection{Adjunctions, Dilations, Erosions}

Dilations and erosions come in pairs as the following concept reveals. Let $\mathcal{L}$ and $\mathcal{M}$ be two complete lattices, and consider two operators $\delta: \mathcal{L} \rightarrow \mathcal{M}$ and $\varepsilon: \mathcal{M} \rightarrow \mathcal{L}$. The operator pair $(\varepsilon, \delta)$ is called an adjunction ${ }^{9}$ between $\mathcal{L}$ and $\mathcal{M}$ if

$$
\delta(X) \leq Y \Longleftrightarrow X \leq \varepsilon(Y) \quad \forall X \in \mathcal{L}, Y \in \mathcal{M}
$$

If $\mathcal{L}=\mathcal{M}$, then the above operator pair is called an adjunction on $\mathcal{L}$. In any adjunction $(\varepsilon, \delta), \varepsilon$ is called the adjoint erosion (or left adjoint) of $\delta$, whereas $\delta$ is the adjoint dilation (or right adjoint) of $\varepsilon$. There is a one-to-one correspondence between the two operators of an adjunction pair, as explained below.

\footnotetext{
${ }^{9}$ As explained in Heijmans and Ronse (1990) and Heijmans (1994), the 'adjunction' is related to a well-known concept in group and lattice theory, the 'Galois connection'. The term 'adjunction' was used in Gierz et al. (1980). It is also related to residuation theory (Blyth and Janowitz, 1972), where we have an increasing operator $\psi$ and find its 'residual' $\psi^{+}$such that $\psi \psi^{+} \leq \mathbf{i d} \leq \psi^{+} \psi$. Then $\psi$, which is called the 'residuated mapping', plays the role of the dilation whereas $\psi^{+}$plays the role of the erosion in the adjunction $\left(\psi^{+}, \psi\right)$. Banon and Barrera (1993) introduced a more general form of adjunction for non-increasing operators.
} 
Proposition 4 (Serra, 1988; Heijmans and Ronse, 1990).

Let $\mathcal{L}$ and $\mathcal{M}$ be two complete lattices. Then:

(a) Given a dilation $\delta: \mathcal{L} \rightarrow \mathcal{M}$, there is a unique erosion

$$
\varepsilon_{\delta}(Y)=\bigvee\{X \in \mathcal{L}: \delta(X) \leq Y\}
$$

such that $\left(\varepsilon_{\delta}, \delta\right)$ is adjunction.

(b) Conversely, given an erosion $\varepsilon: \mathcal{M} \rightarrow \mathcal{L}$, there is a unique dilation

$$
\delta_{\varepsilon}(X)=\bigwedge\{Y \in \mathcal{M}: X \leq \varepsilon(Y)\}
$$

such that $\left(\varepsilon, \delta_{\varepsilon}\right)$ is adjunction.

The following summarizes some properties of adjunctions. The proposition is adapted to our definitions.

Proposition 5 (Serra, 1988; Heijmans and Ronse, 1990).

Let $(\varepsilon, \delta)$ be and adjunction between complete lattices $\mathcal{L}$ and $\mathcal{M}$. Then:

(a) $\delta$ is a dilation and $\varepsilon$ is an erosion.

(b) $\delta \varepsilon$ is an opening, and $\varepsilon \delta$ is a closing.

(c) If $\left(\varepsilon_{j}, \delta_{j}\right)$ are adjunctions between $\mathcal{L}$ and $\mathcal{M}$, then $\left(\bigwedge_{j} \varepsilon_{j}, \bigvee_{j} \delta_{j}\right)$ is an adjunction between $\mathcal{L}$ and $\mathcal{M}$.

(d) If $\left(\varepsilon_{1}, \delta_{1}\right)$ and $\left(\varepsilon_{2}, \delta_{2}\right)$ are adjunctions between $\mathcal{L}$ and $\mathcal{M}$, then $\varepsilon_{2} \leq \varepsilon_{1}$ iff $\delta_{2} \geq \delta_{1}$.

(e) If $\phi: \mathcal{L} \rightarrow \mathcal{M}$ is a lattice automorphism, then $\left(\phi^{-1}, \phi\right)$ is an adjunction between $\mathcal{L}$ and $\mathcal{M}$.

(f) If $\left(\varepsilon_{1}, \delta_{1}\right)$ is an adjunction between $\mathcal{L}$ and $\mathcal{M}$ and $\left(\varepsilon_{2}, \delta_{2}\right)$ is an adjunction between complete lattices $\mathcal{M}$ and $\mathcal{N}$, then $\left(\varepsilon_{1} \varepsilon_{2}, \delta_{2} \delta_{1}\right)$ is an adjunction between $\mathcal{L}$ and $\mathcal{N}$.

(g) If $\mathcal{L}$ and $\mathcal{M}$ have negations $(\cdot)^{*}$, then $(\varepsilon, \delta)$ is an adjunction between $\mathcal{L}$ and $\mathcal{M}$ if and only if $\left(\delta^{*}, \varepsilon^{*}\right)$ is an adjunction between $\mathcal{M}$ and $\mathcal{L}$.

Example 7 (Adjunctions of Minkowski Erosions and Dilations):

(a) Adjunction of Minkowski Set Erosion and Dilation:

On the complete lattice of the power set of $\mathbb{R}^{m}$ or $\mathbb{Z}^{m}$, consider the Minkowski set dilation by a structuring set $B$ :

$$
\delta_{B}(X)=X \oplus B=\bigcup_{\mathbf{b} \in B} X_{+\mathbf{b}}
$$

Its adjoint erosion $\varepsilon_{B}$ must satisfy

$$
X \oplus B \subseteq Y \Longleftrightarrow X \subseteq \varepsilon_{B}(Y)
$$

Expressing in the LHS the set dilation $X \oplus B$ as union of translates of $X$ yields

$$
\begin{aligned}
\bigcup_{\mathbf{b} \in B} X_{+\mathbf{b}} \subseteq Y & \Longleftrightarrow X X_{+\mathbf{b}} \subseteq Y \forall \mathbf{b} \in B \\
& \Longleftrightarrow X \subseteq Y_{-\mathbf{b} \forall \mathbf{b} \in B} \\
& \Longleftrightarrow X \subseteq \bigcap_{\mathbf{b} \in B} Y_{-\mathbf{b}} \\
& \Longleftrightarrow X \subseteq Y \ominus B
\end{aligned}
$$

Hence, the adjoint erosion $\varepsilon_{B}$ must equal the Minkowski set erosion by $B$ :

$$
\varepsilon_{B}(Y)=Y \ominus B
$$

(b) Adjunction of Minkowski Flat Erosion and Dilation:

On the complete lattice of extended real-valued functions defined on $\mathbb{E}^{m}=\mathbb{R}^{m}$ or $\mathbb{Z}^{m}$, consider the 
Minkowski flat ${ }^{10}$ erosion of a function $g(\mathbf{x})$ by a structuring set element $B \subseteq \mathbb{E}^{m}$ :

$$
\varepsilon_{B}(g)(\mathbf{x})=(g \ominus B)(\mathbf{x}) \triangleq \bigwedge_{\mathbf{y} \in B} g(\mathbf{x}+\mathbf{y})
$$

Its adjoint dilation $\delta_{B}$ must satisfy

$$
\delta_{B}(f) \leq g \Longleftrightarrow f \leq g \ominus B
$$

By expressing the flat erosion as infimum of translates of $g$, the RHS becomes

$$
\begin{aligned}
f(\mathbf{x}) \leq \bigwedge_{\mathbf{y} \in B} g(\mathbf{x}+\mathbf{y}) & \Longleftrightarrow f(\mathbf{x}) \leq g(\mathbf{x}+\mathbf{y}) \forall \mathbf{y} \in B, \forall \mathbf{x} \\
& \Longleftrightarrow f(\mathbf{x}-\mathbf{y}) \leq g(\mathbf{x}) \forall \mathbf{y} \in B, \forall \mathbf{x} \\
& \Longleftrightarrow \bigvee_{\mathbf{y} \in B} f(\mathbf{x}-\mathbf{y}) \leq g(\mathbf{x}) \forall \mathbf{x} \\
& \Longleftrightarrow f \oplus B \leq g
\end{aligned}
$$

Hence, the adjoint dilation must equal the Minkowski flat dilation by $B$ :

$$
\delta_{B}(f)(\mathbf{x})=(f \oplus B)(\mathbf{x}) \triangleq \bigvee_{\mathbf{y} \in B} f(\mathbf{x}-\mathbf{y})
$$

(c) Adjunction of Minkowski Weighted Erosion and Dilation:

On the complete lattice of extended real-valued functions defined on $\mathbb{E}^{m}=\mathbb{R}^{m}$ or $\mathbb{Z}^{m}$, consider the Minkowski weighted dilation of a function $f(\mathbf{x})$ by a structuring function kernel $k(\mathbf{x})$ :

$$
\delta_{k}(f)(\mathbf{x})=(f \oplus k)(\mathbf{x}) \triangleq \bigvee_{\mathbf{y} \in \mathbb{E}^{m}} f(\mathbf{x}-\mathbf{y})+k(\mathbf{y})=\bigvee_{\mathbf{z} \in \mathbb{E}^{m}} f(\mathbf{z})+k(\mathbf{x}-\mathbf{z})
$$

This is also called the supremal convolution of $f$ and $k$ in convex analysis and optimization (Bellman and Karush, 1963; Rockafellar, 1970). Its adjoint erosion $\varepsilon_{k}$ must satisfy

$$
f \oplus k \leq g \Longleftrightarrow f \leq \varepsilon_{k}(g)
$$

Hence, the adjoint erosion must equal the Minkowski weighted erosion by $k$ :

$$
\varepsilon_{k}(g)(\mathbf{x})=(g \ominus k)(\mathbf{x}) \triangleq \bigwedge_{y \in \mathbb{E}^{m}} g(\mathbf{x}+\mathbf{y})-k(\mathbf{y})=\bigwedge_{\mathbf{z} \in \mathbb{E}^{m}} g(\mathbf{z})-k(\mathbf{x}+\mathbf{z})
$$

\subsection{Shift-varying Dilation and Erosion}

In applying image operators to non-stationary signals, the need often arises to vary the moving computational kernel and adapt it at different locations based on various strategies. One such possibility is to have a family of kernels possibly varying at each location. This leads to spatial adaptation, i.e. spatially-varying operators, also called shift-varying operators. These ideas are practically useful both for linear and morphological operators. In the latter case, they form cases of adaptive morphology; in this area, a basic requirement on developing adaptive morphological operators should be on keeping the adjunction structure in pairs of adaptive dilations and erosions. Next, we summarize the main ideas and give some examples.

\footnotetext{
${ }^{10} \mathrm{An}$ operator is called flat if the range of its output signals is equal to or a subset of the range of its input signals; hence, if its input is binary, the output will also be binary. Thus, a flat operator can process both graylevel and binary images without altering this property of the input.
} 


\subsubsection{Structuring Element Map (SEM)}

A general framework for spatially-varying morphology, in the Euclidean space, is the concept of the structuring element map (SEM), also known as 'structuring function', proposed in (Serra, 1988, chap.2). In this case, we have not a fixed but a spatially-varying (SV) structuring element, which can be either a set or a function. For example, in the case of structuring sets we have a map $\mathcal{A}(x): E \rightarrow \mathcal{P}(E)$ that assigns a possibly different set $\mathcal{A}(x)$ at each point $x$ of space $E$. The SEM is equivalent to the datum of any dilation operator, as explained by the following.

Theorem 9 (Serra, 1988).

$A$ set operator $\delta$ on the collection $\mathcal{P}(E)$ of all subsets of some general domain $E$ is a dilation if and only if there exists a map $\mathcal{A}(x): E \rightarrow \mathcal{P}(E)$ such that $\delta$ can be expressed as

$$
\delta_{\mathcal{A}}(X)=\bigcup_{x \in X} \mathcal{A}(x)
$$

To find the adjoint erosion, $\varepsilon_{\mathcal{A}}$, of $\delta_{\mathcal{A}}$ in $(62)$, first note that

$$
\bigcup_{x \in X} \mathcal{A}(x) \subseteq Y \Longleftrightarrow \mathcal{A}(x) \subseteq Y \forall x \in X
$$

Hence, the adjoint erosion is

$$
\varepsilon_{\mathcal{A}}(Y)=\{x \in E: \mathcal{A}(x) \subseteq Y\}
$$

The SEM approach has also been extended to morphological operators on graylevel image functions (Serra, 1988, chap.9). This can be done in two possible ways:

(1) Adaptive Window, where the operators are flat and use an SV set-valued SEM $\mathcal{A}: E \rightarrow \mathcal{P}(E)$. For example, consider the following SV flat dilation and erosion:

$$
\begin{aligned}
& \delta_{\mathcal{A}}(f)(\mathbf{x})=\bigvee_{\mathbf{y} \in \mathcal{A}(\mathbf{x})} f(\mathbf{x}-\mathbf{y}) \\
& \varepsilon_{\mathcal{A}}(f)(\mathbf{x})=\bigwedge_{\mathbf{y} \in \mathcal{A}(\mathbf{x})} f(\mathbf{x}+\mathbf{y})
\end{aligned}
$$

Each of them is a valid lattice erosion and dilation, and their pair $\left(\varepsilon_{\mathcal{A}}, \delta_{\mathcal{A}}\right)$ is an adjunction of SV morphological operators.

Example 8 One example of shift-varying dilation and erosion that is very useful in practice arises when we have to dilate or erode a signal $f(x)$ by a constant window $B$, but the values of $f$ are given only over a compact mask (frame) $S$ and there is no knowledge of its values outside $S$. The problem arises when the moving window $B$ reaches the boundaries of $S$ and includes points from the mask complement $S^{c}$. Then, one solution is to compute the local maximum or minimum of signals values only over the intersection of the moving $B$ and the analysis frame $S$, so that there will never be a need to use values of $f$ outside $S$. This is formalized as a shift-varying Minkowski dilation or erosion by a shift-varying structuring element

$$
\mathcal{A}(x)=S \cap B_{+x}
$$

The above solution creates an output signal having the same extent as the input.

(2) Adaptive Kernel, where the weighted dilation and erosion operators use an SV gray kernel $k: \mathbb{E}^{m} \rightarrow \operatorname{Fun}\left(\mathbb{E}^{m}, \overline{\mathbb{R}}\right)$, whose support and/or weights/values may vary at each point $\mathbf{x}$ of the image domain:

$$
\begin{aligned}
\Delta_{k}(f)(\mathbf{x}) & =\bigvee_{\mathbf{y}} f(\mathbf{x}-\mathbf{y})+k_{\mathbf{x}}(\mathbf{y}) \\
\mathcal{E}_{k}(f)(\mathbf{x}) & =\bigwedge_{\mathbf{y}} f(\mathbf{x}+\mathbf{y})-k_{\mathbf{x}}(\mathbf{y})
\end{aligned}
$$


Thus, we have replaced the shift-varying flat structuring element $\mathcal{A}(\mathbf{x})$ with a shift-varying weighted structuring element $k_{\mathbf{x}}(\cdot)$. The mapping $\mathbf{x} \mapsto k_{\mathbf{x}}$ corresponds to each point in the signal domain $\mathbb{E}^{m}$ a possibly different function from $\operatorname{Fun}\left(\mathbb{E}^{m}, \overline{\mathbb{R}}\right)$. The support of each such function $k_{\mathbf{x}}$ is a shiftvarying window $\mathcal{A}(\mathbf{x})$. This function-valued SEM is also called the impulse response map in a later section on translation-invariant operators, because each structuring function $k_{\mathbf{x}}$ can be seen as the 'impulse response' of a dilation system when excited by a properly defined 'impulse signal'.

\subsubsection{Adaptive Morphology}

Since the introduction of the concept of the SEM approach, the interest of the scientific community for adaptive morphology has continuously increased. Examples include the following:

1) The SV structuring element idea was explored in Lerallut et al. (2005) by developing morphological operators based on adjunctions with non-fixed shape kernels. These filters were able to adapt their kernel shape by taking into consideration the local image contour variations and gave better results than shift-invariant filters that used fixed-shape kernels. Due to their adaptive nature, they were called amoeba filters.

2) Adaptivity is omnipresent in connected morphological operators (Salembier and Serra, 1995; Ouzounis and Wilkinson, 2007), which process connected components in images. For instance, area openings (Vincent, 1992; Cheng and Venetsanopoulos, 1992) at scale $\lambda$ are geometry-adaptive filters: the size of the structuring element is linked to the area $\lambda$ of the connected components of image.

3) Adaptive Neighborhoods: In Braga-Neto (1996) and in Debayle and Pinoli (2006), given some criterion mapping $h$ (expressing local radiometric, morphological, or geometrical information) and a tolerance $t>0$, at each point $\mathbf{x} \in \mathbb{E}^{m}$, an adaptive neighborhood $V_{t}^{h}(\mathbf{x})$ is defined that contains all points $\mathbf{y}$ with $|h(\mathbf{y})-h(\mathbf{x})| \leq t$ and is connected. Obviously, its shape and size vary spatially and adapt to the local image characteristics around the seed point. Then, one can build a SEM that provides an auto-reflected collection of adaptive structuring sets

$$
\mathcal{A}(\mathbf{x})=\bigcup_{\mathbf{z} \in \mathbb{E}^{m}}\left\{V_{t}^{h}(\mathbf{z}): \mathbf{x} \in V_{t}^{h}(\mathbf{z})\right\}
$$

and use this to construct SV dilations and erosions.

4) Kernel and Basis Representation for SV operators: Bouaynaya et al. (2008) and Bouaynaya and Schonfeld (2008) developed representations for SV morphological systems, which only possess the increasing property, as suprema and infima of SV erosions and dilations respectively.

A brief overview of the above and other approaches for adaptive morphology has been presented in Maragos and Vachier (2009). An important issue is whether the pairs of adaptive dilations and erosions proposed by various researchers are actually lattice adjunctions; this is examined by Roerdink (2009).

\subsection{Convergence and Continuity on Lattices}

\subsubsection{Convergence and Continuity on Topological Image Spaces}

Let $E$ be topological space that is:

(1) locally compact (i.e. each point has a compact neighborhood),

(2) Hausdorff (i.e. two distinct points can be separated by two disjoint neighborhoods), and

(3) second countable, i.e. has a countable basis ${ }^{11}$ (i.e. every open set can be expressed as a countable union of basis sets).

Examples of such topological spaces $E$ are the continuous plane $\mathbb{R}^{2}$ with the Euclidean topology and the discrete plane $\mathbb{Z}^{2}$ with the discrete topology. Consider now the collection $\mathcal{F}(E)$ of the closed

\footnotetext{
${ }^{11}$ In a topological space, the term 'topology' means the collection $\mathcal{G}$ all open subsets. The term 'basis' means a subcollection of $\mathcal{G}$ such that every open set is a union of basis sets.
} 
subsets of $E$, which model binary image objects. Equipped with the hit-miss topology (Matheron, $1975), \mathcal{F}(E)$ becomes a compact, Hausdorff space with countable basis.

Given a set sequence $\left(X_{n}\right)$ in $\mathcal{F}(E)$, define its upper limit $\overline{\lim } X_{n}$ as the set of all points to which converges some subsequence of points $x_{n_{k}} \in X_{n_{k}}$ and its lower limit $\underline{\lim } X_{n}$ as the set of all points to which converges some sequence of points $x_{n} \in X_{n}$. These two limits obey the inequality

$$
\underline{\lim } X_{n} \subseteq \varlimsup \lim X_{n}
$$

If $\underline{\lim } X_{n}=\varlimsup X_{n}=X$, then we say that the sequence $\left(X_{n}\right)$ converges to the limit $X$ and we write $\lim X_{n}=X$. Incidentally, this $\operatorname{limit} X$ is a closed set.

Consider now a set operator $\psi$ on $\mathcal{F}(E)$. It is called upper semi-continuous (u.s.c.) if $\overline{\lim } X_{n} \subseteq$ $\psi(X)$ for each convergent set sequence $\left(X_{n}\right)$ with $\lim X_{n}=X$. Similarly, $\psi$ is called lower semicontinuous (l.s.c.) if $\psi(X) \subseteq \underline{\lim } X_{n}$. Obviously, $\psi$ is continuous if it is both u.s.c. and l.s.c.

The above concepts of topological semi-convergence and semi-continuity can be extended to topological spaces of functions, specifically to the $\operatorname{space} \operatorname{Fun}_{u s c}(E, \overline{\mathbb{R}})$ of extended real-valued u.s.c. functions and to operators on this signal space. Note this image signal space is related to the image set space $\mathcal{F}(E)$ because a function is u.s.c. if and only if all its level sets are closed sets.

Proposition 6 (Matheron, 1975; Serra, 1982).

Consider set operators on $\mathcal{F}(E)$. Then:

(a) The Minkowski set dilation by compact structuring elements is continuous.

(b) The Minkowski set erosion, opening and closing by compact structuring elements are upper semi-continuous.

\subsubsection{Order Convergence and Continuity on Lattices}

The Euclidean topology of the reals $\mathbb{R}$ can be fully defined by using only the order $\leq$. As explained in Birkhoff (1967), this can be generalized to any chain $(\mathcal{C}, \leq)$ by defining 'open intervals' as we do on $\mathbb{R}$; i.e. for any $a, b \in \mathcal{C}$ we define

$$
\begin{aligned}
(-\infty, a) & \triangleq\{x \in \mathcal{C}: x<a\} \\
(b,+\infty) & \triangleq\{x \in \mathcal{C}: x>b\} \\
(a, b) & \triangleq\{x \in \mathcal{C}: a<x<b\}
\end{aligned}
$$

If we also need 'closed intervals', this can be easily done by replacing $<$ with $\leq$; e.g. we assume a complete chain and define the closed intervals

$$
[-\infty, a] \triangleq\{x \in \mathcal{C}: O \leq x \leq a\}, \quad[b,+\infty] \triangleq\{x \in \mathcal{C}: b \leq x \leq I\}
$$

By using these intervals we can now define order-based convergence and continuity on any chain. For example, if $\left(x_{n}\right)$ is a sequence in a chain, we can write $x_{n} \rightarrow a$ to mean that for any open interval $(b, c)$ around $a$ there exists some $n_{0}$ such that $x_{n} \in(b, c)$ for all $n \geq n_{0}$; exactly as we do for the topological convergence on $\mathbb{R}$.

Now this order-based convergence on a chain can be split into a conjunction of two conditions: $\lim \sup x_{n} \leq a$ and $\lim \inf x_{n} \geq a$, where the limsup and liminf can be expressed as sup/inf combinations:

$$
\begin{aligned}
\lim \sup X_{n} & \triangleq \bigwedge_{n \geq 1} \bigvee_{k \geq n} X_{k} \\
\liminf X_{n} & \triangleq \bigvee_{n \geq 1} \bigwedge_{k \geq n} X_{k}
\end{aligned}
$$

The minimax inequality on lattices (Birkhoff, 1967) implies that

$$
\liminf X_{n} \leq \limsup X_{n}
$$


In the above definitions we replaced the sequence of elements in a chain with an arbitrary sequence $\left(X_{n}\right)$ of lattice elements, so that we can extend henceforth the two limit definitions $(73,74)$ to any sequence $\left(X_{n}\right)$ in a general complete lattice and use them to define the following order-based convergence and continuity in lattices. Specifically, by mimicking a result on convergence of a sequence $\left(x_{n}\right)$ in topological space where $x_{n} \rightarrow x$ iff $\lim \sup x_{n}=\liminf x_{n}=x$, a sequence $\left(X_{n}\right)$ in a complete lattice $\mathcal{L}$ is defined to order converge to a lattice element $X$, written as $X_{n} \stackrel{\text { ord }}{\rightarrow} X$, if $\liminf X_{n}=\lim \sup X_{n}=X$.

Now we also define order continuity: An operator $\psi: \mathcal{L} \rightarrow \mathcal{M}$ between two complete lattices is called $\downarrow$-continuous if $\left(X_{n}\right) \stackrel{\text { ord }}{\rightarrow} X$ in $\mathcal{L}$ implies that $\lim \sup \psi\left(X_{n}\right) \leq \psi(X)$ in $\mathcal{M}$. Dually, $\psi$ is called $\uparrow$-continuous if $\left(X_{n}\right) \stackrel{\text { ord }}{\rightarrow} X$ implies that $\liminf \psi\left(X_{n}\right) \geq \psi(X)$. Finally, $\psi$ is called $\uparrow$-continuous or order continuous if is both $\downarrow$-continuous and $\uparrow$-continuous. Obviously, on a chain the concepts of order convergence and order continuity coincide with their topological counterparts.

There is a stronger form of order convergence applicable to monotone sequences and increasing operators. In a complete lattice $\mathcal{L}$, we write $X_{n} \downarrow X$ to mean a monotonic convergence where $\left(X_{n}\right)$ is a decreasing sequence $\left(X_{n+1} \leq X_{n}\right)$ and $X=\bigwedge_{n} X_{n}$. Dually, we write $X_{n} \uparrow X$ to mean that $\left(X_{n}\right)$ is an increasing sequence $\left(X_{n+1} \geq X_{n}\right)$ and $X=\bigvee_{n} X_{n}$. Based on this monotonic convergence, we can easily examine the order continuity of increasing operators, as explained next.

Proposition 7 (Heijmans, 1994).

(a) If $\left(X_{n}\right)$ is a monotone sequence in a complete lattice with $X_{n} \downarrow X$ or $X_{n} \uparrow X$, then $X_{n} \stackrel{\text { ord }}{\rightarrow} X$. (b) An increasing operator $\psi: \mathcal{L} \rightarrow \mathcal{M}$ between two complete lattices is $\downarrow$-continuous if and only if $X_{n} \downarrow X$ implies that $\psi\left(X_{n}\right) \downarrow \psi(X)$ for any sequence $\left(X_{n}\right)$. Dually, an increasing operator $\psi$ is $\uparrow$-continuous iff $X_{n} \uparrow X$ implies that $\psi\left(X_{n}\right) \uparrow \psi(X)$.

The above result establishes that the general order convergence and continuity become equivalent to the monotonic convergence and continuity as long as we work on a complete lattice and the operator is increasing. Now if we focus on specific classes of increasing operators like erosions and dilations, some of the following results are immediate consequences of their distributivity over infima and/or suprema.

Proposition 8 (Heijmans, 1994).

(a) Erosions are $\downarrow$-continuous.

(b) Dilations are $\uparrow$-continuous.

(c) Automorphisms are $\uparrow$-continuous.

(c) The infimum of $\downarrow$-continuous operators is $\downarrow$-continuous. The supremum of $\uparrow$-continuous operators is $\uparrow$-continuous.

(d) The $\psi \phi$ composition of two operators is $\downarrow$-continuous if (i) $\phi$ is $\downarrow$-continuous and $\psi$ is increasing and $\downarrow$-continuous, or (ii) $\phi$ is $\downarrow$-continuous and $\psi$ is $\downarrow$-continuous.

Finally, the following result connects the topological semi-continuity with the order-based monotonic continuity.

Proposition 9 (Matheron, 1975).

Consider an increasing operator on the space $\mathcal{F}(E)$ of closed subsets of a locally compact, Hausdorff, second countable topological space $E$. Then, $\psi$ is u.s.c. if and only if it is $\downarrow$-continuous.

\subsection{Openings, Closings, Order Projections}

A large variety of useful morphological operators share two properties: increasing and idempotent. Such operators were called morphological filters by Matheron and Serra. We shall call them order projections, since they preserve the lattice ordering and are idempotent in analogy with the linear projections that preserve the algebraic structure of linear spaces and are idempotent. The most well-studied special cases of order projections are the lattice openings and closings, each of which 
has an additional property. Specifically, openings are order projections that are anti-extensive, whereas closings are extensive order projections.

Combinations of such generalized filters have proven to be very useful for image enhancement, multiscale image simplification, segmentation, motion analysis, and object detection.

In this section we focus on their lattice theoretic properties and representations. To build intuition, we also mention some examples and show figures with image analysis experiments.

\subsubsection{Lattice Theory of Openings and Closings}

Several algebraic properties of order projections, and especially those of openings and closings, can be analyzed based on the set of fixed points of such an operator $\psi$, called the invariance domain and denoted by

$$
\operatorname{Inv}(\psi) \triangleq\{X \in \mathcal{L}: \psi(X)=X\}
$$

The following summarizes some immediate properties of the invariance domain stemming from various characteristic of its corresponding operator.

Proposition 10 (Matheron, 1975; Heijmans, 1994).

Let $\psi$ be an operator on a complete lattice $\mathcal{L}$ and let $\operatorname{Inv}(\psi)$ be its invariance domain. Then:

(a) (Tarski Fixpoint Theorem): If $\psi$ is increasing, then $\operatorname{Inv}(\psi)$ is nonempty.

(b) $\psi$ is idempotent if and only if $\operatorname{Inv}(\psi)=\operatorname{Ran}(\psi)$.

(c) If $\psi$ is increasing and anti-extensive, then $\operatorname{Inv}(\psi)$ is sup-closed.

(d) If $\psi$ is increasing and extensive, then $\operatorname{Inv}(\psi)$ is inf-closed.

We see from the above proposition, part (c) and (d), that, the invariance domains of increasing extensive and antiextensive operators are complete lattices. If we add the third property of idempotence, we get openings and closings. Well, the fixed points of an opening (resp. closing) contain sufficient information to represent them via a supremum (resp. infimum), as the following important result explains.

Proposition 11 (Serra, 1988; Ronse and Heijmans, 1991).

If $\alpha$ and $\beta$ are opening and closing operators, respectively, on a complete lattice $\mathcal{L}$, then:

$$
\begin{array}{ll}
\alpha(X)=\bigvee\{Y \in \operatorname{Inv}(\alpha): Y \leq X\}, & X \in \mathcal{L} \\
\beta(X)=\bigwedge\{Y \in \operatorname{Inv}(\beta): Y \geq X\}, & X \in \mathcal{L}
\end{array}
$$

Thus, the invariance domain can uniquely represent an opening or closing. The following proposition groups together some useful facts about openings and closings that are formed from adjunctions.

Proposition 12 (Serra, 1988; Ronse and Heijmans, 1991; Heijmans, 1994).

Let $(\varepsilon, \delta)$ be an adjunction between two complete lattices. Then:

(a) $\delta \varepsilon$ is an opening, and $\varepsilon \delta$ is a closing.

(b) $\delta \varepsilon \delta=\delta$ and $\varepsilon \delta \varepsilon=\varepsilon$.

(c) $\operatorname{Inv}(\delta \varepsilon)=\operatorname{Ran}(\delta)$ and $\operatorname{Inv}(\varepsilon \delta)=\operatorname{Ran}(\varepsilon)$.

Thus, from any adjunction $(\varepsilon, \delta)$ we can always construct an opening and a closing via the composition of its erosion and dilation. The converse is also true: given an opening or closing we can express it by composing the erosion and dilation of some properly defined adjunction. This is explained next for the case of an opening. 
Proposition 13 (Heijmans, 1994).

Any opening $\alpha$ on a complete lattice $\mathcal{L}$ can be can be expressed as the composition $\delta \varepsilon$ of an erosion with a dilation, where $(\varepsilon, \delta)$ is an adjunction between $\mathcal{L}$ and the complete lattice $\mathcal{M}=\mathcal{P}(\operatorname{Inv}(\alpha))$. The erosion $\varepsilon: \mathcal{L} \rightarrow \mathcal{M}$ is defined by

$$
\varepsilon(X)=\{A \in \operatorname{Inv}(\alpha): A \leq X\}, \quad X \in \mathcal{L}
$$

The dilation $\delta: \mathcal{M} \rightarrow \mathcal{L}$ is defined by

$$
\delta(\mathcal{K})=\bigvee \mathcal{K}, \quad \mathcal{K} \subseteq \operatorname{Inv}(\alpha)
$$

The following result allows to synthesize new openings (resp. closings) by taking the supremum (resp. infimum) of a family of openings (resp. closings).

Proposition 14 (Serra, 1988; Ronse and Heijmans, 1991; Heijmans, 1994).

Let $\left\{\alpha_{i}\right\}$ and $\left\{\beta_{i}\right\}$ be indexed families of openings and closings on a complete lattice. Then:

(a) $\alpha=\bigvee_{i} \alpha_{i}$ is an opening and $\beta=\bigwedge_{i} \beta_{i}$ is a closing.

(b) $\operatorname{Inv}(\alpha)=\bigcup_{i} \operatorname{Inv}\left(\alpha_{i}\right)$ and $\operatorname{Inv}(\beta)=\bigcap_{i} \operatorname{Inv}\left(\beta_{i}\right)$.

\subsubsection{Examples of Openings and Closings}

Thus, from any adjunction we can generate an opening via the composition of its erosion and dilation. As an elementary example, if we consider the translation-invariant (Minkowski) morphological erosion $\varepsilon(X)=X \ominus B$ and dilation $\delta(X)=X \oplus B$, then $\delta \varepsilon(X)$ coincides with the translation-invariant (Minkowski) morphological opening $X \circ B=(X \ominus B) \oplus B$. But there are also numerous other possibilities, some of which are briefly described next.

Radial Openings: Consider a 2D image $f$ that contains 1D objects, e.g. lines; then the simple Minkowski opening or closing of $f$ by a disk $B$ will eliminate these 1D objects. Another problem arises when $f$ contains large-scale objects with sharp corners that need to be preserved; in such cases opening or closing $f$ by a disk $B$ will round these corners. These two problems could be lessened or avoided in some cases if we replace the conventional opening with a radial opening

$$
\alpha_{L}(f)=\bigvee_{\theta} f \circ L_{\theta}
$$

where the sets $L_{\theta}$ are rotated versions of a symmetric line segment $L$ at various angles $\theta \in[0,2 \pi)$. This has the effect of preserving an object in $f$ if this object is left unchanged after the opening by $L_{\theta}$ in at least one of the possible orientations $\theta$. See Fig. 2 for an example of a radial opening of a graylevel image. Dually, in case of dark 1D objects, we can use a radial closing

$$
\beta_{L}^{*}(f)=\bigwedge_{\theta} f \bullet L_{\theta}=-\alpha_{L}(-f)
$$

Connected Filters: The flat zones of an image signal $f: \mathbb{E}^{m} \rightarrow \overline{\mathbb{R}}$ are defined as the connected components of the image domain on which $f$ assumes a constant value. A useful class of morphological filters was introduced in Salembier and Serra (1995) which operate by merging flat zones and hence exactly preserving the contours of the image parts remaining in the filter's output. These are called connected operators. They cannot create new image structures or new boundaries if they did not exist in the input. Specifically, if $\mathcal{D}$ is a partition of the image domain, let $\mathcal{D}(x)$ denote the (partition member) region that contains the pixel $x$. Now, given two partitions $\mathcal{D}_{1}, \mathcal{D}_{2}$, we say that $\mathcal{D}_{1}$ is 'finer' than $\mathcal{D}_{2}$ if $\mathcal{D}_{1}(x) \subseteq \mathcal{D}_{2}(x)$ for all $x$. An operator $\psi$ is called connected if the flat zone partition of its input $f$ is finer than the flat zone partition of its output $\psi(f)$. Next we discuss two types of connected operators, the area filters and the reconstruction filters, which are lattice openings or closings. 


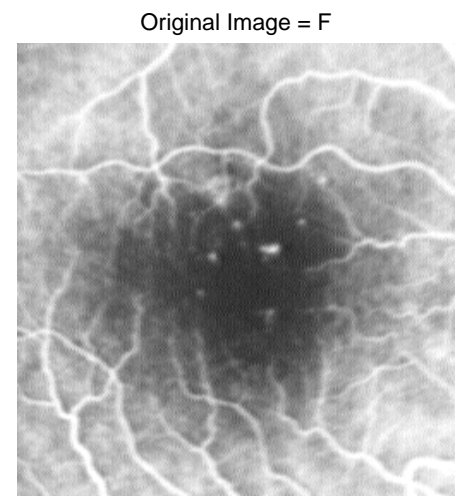

(a)

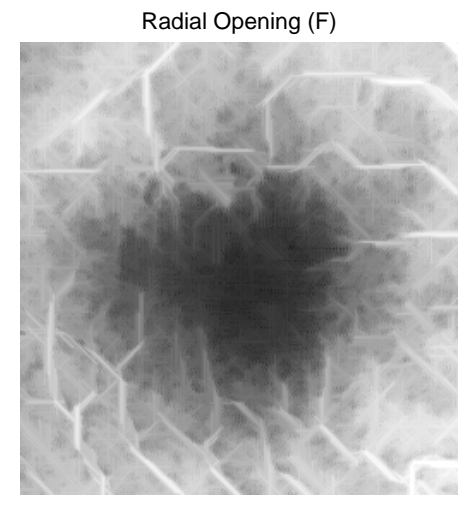

(b)

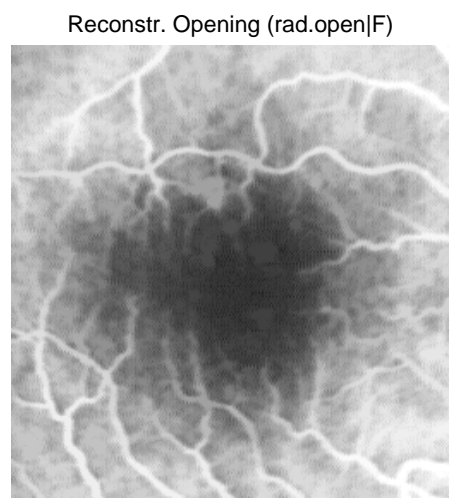

(c)

Figure 2: (a) Original image $f$ of an eye angiogram with microaneurisms $(264 \times 255$ pixels). (b) Radial opening $\alpha_{L}(f)$ of $f$ as max of four openings by lines oriented at $0^{\circ}, 45^{\circ}, 90^{\circ}, 135^{\circ}$ of size 20 pixels each. (c) Reconstruction opening $\varrho^{-}\left(\alpha_{L}(f) \mid f\right)$ of $f$ using the radial opening as marker.

Area Filters: There are numerous image enhancement problems where what is needed is suppression of arbitrarily-shaped connected components in the input image whose areas (number of pixels) are smaller than a certain threshold $n$. This can be accomplished by the area opening $\alpha_{n}$ of size $n$ which, for binary images, keeps only the connected components whose area is $\geq n$ and eliminates the rest. Consider an input set $X=\bigsqcup_{i} X_{i}$ as a union of disjoint connected components $X_{i}$. Then the output from the area opening is

$$
\alpha_{n}(X)=\bigsqcup_{\operatorname{area}\left(X_{j}\right) \geq n} X_{j}, \quad X=\bigsqcup_{i} X_{i}
$$

where $\bigsqcup$ denotes disjoint union. The area opening can be extended to graylevel images $f$ by applying the same binary area opening to all level sets of $f$

$$
X_{v}(f) \triangleq\{x: f(x) \geq v\}
$$

and constructing the filtered graylevel image via threshold superposition:

$$
\alpha_{n}(f)(x)=\sup \left\{v: x \in \alpha_{n}\left[X_{v}(f)\right]\right\}
$$

The graylevel area opening is a flat operator. Figure 3 shows examples of binary and gray area openings. If we apply the above operations to the complements of the level sets of an image, we obtain an area closing.

The area filters can be generalized to other connected operators that operate by processing connected components of a binary image (or of the level sets of a graylevel image) and keeping only those components for which an increasing criterion exceeds some threshold. Such filters are called attribute openings and were proposed and studied in Breen and Jones (1996); examples of increasing criteria include (i) the diameter, or area of the largest (resp. smallest) circle that can be inscribed into (resp. circumscribed around) a connected component, (ii) the area or perimeter of the convex hull of a connected component. Generalizations of attribute filters based on secondorder connectivity were studied in Ouzounis and Wilkinson (2007).

Reconstruction Opening and Closing: Consider a reference (image) set $X=\bigsqcup_{i} X_{i}$ as a union of $I$ disjoint connected components $X_{i}, i \in I$, and let $M \subseteq X_{j}$ be a marker in some component(s) $X_{j}$, indexed by $j \in J \subseteq I$; i.e., $M$ could consist of a single point or some feature sets in $X$ that lie only in the component(s) $X_{j}$. Let us define the reconstruction opening as the operator

$$
\varrho^{-}(M \mid X) \triangleq \text { connected components of } X \text { intersecting } M \text {. }
$$




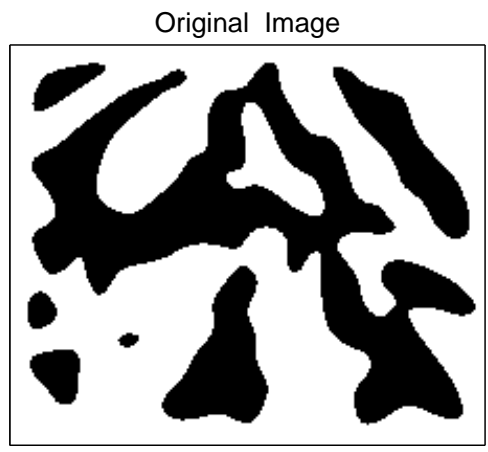

(a)

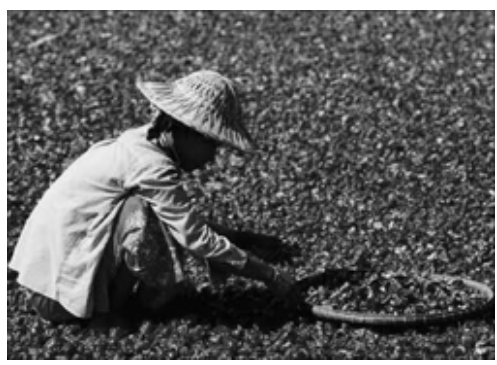

(d)

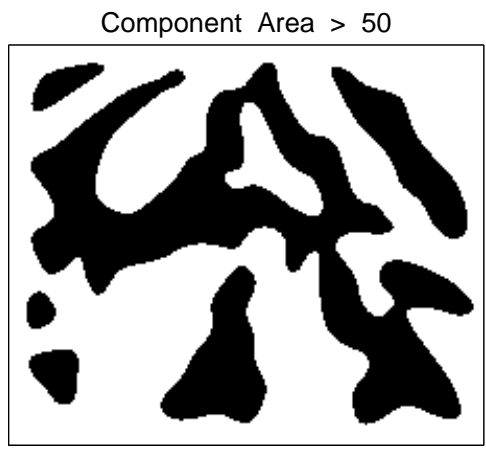

(b)

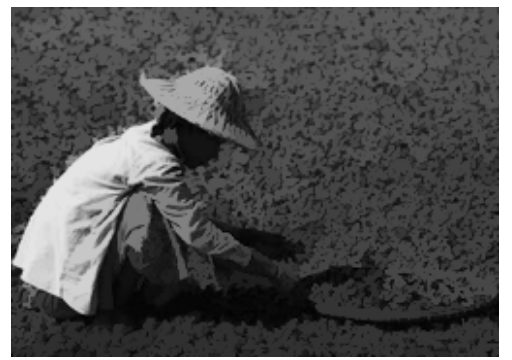

(e)

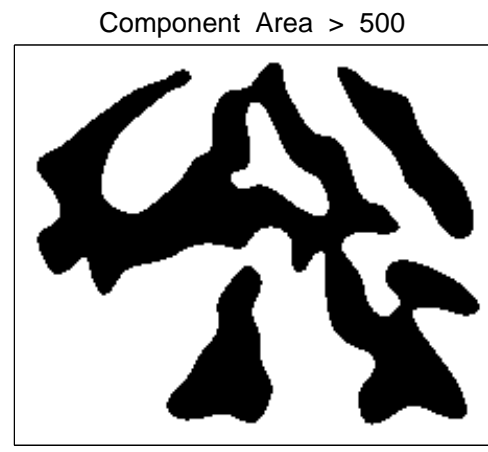

(c)

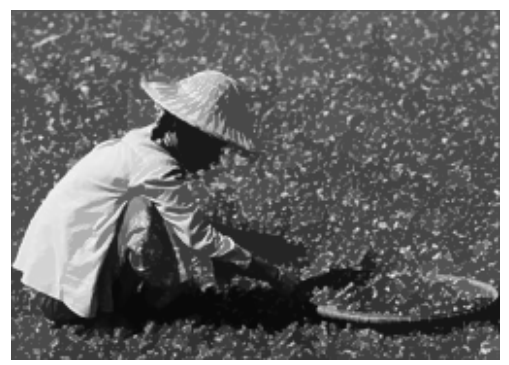

(f)

Figure 3: Top row: (a) Original binary image $(192 \times 228$ pixels). (b) Area opening by keeping connected components with area $\geq 50$. (c) Area opening by keeping components with area $\geq 500$. Bottom row: (d) Gray original image $(420 \times 300$ pixels $)$. (e) Gray area opening by keeping bright components with area $\geq 500$. (f) Gray area closing by keeping dark components with area $\geq 500$.

Its output contains exactly the input component(s) $X_{j}$ that intersect the marker. It can extract large-scale components of the image from knowledge only of a smaller marker inside them. Note that the reconstruction opening has two inputs. If the marker $M$ is fixed, then the mapping $X \mapsto \varrho^{-}(M \mid X)$ is a lattice opening since it is increasing, anti-extensive and idempotent. Its output is called the morphological reconstruction of (the components of) $X$ from the marker $M$.

For both continuous and discrete shapes, the reconstruction can be modeled as the union of multiscale geodesic dilations of the marker $M$ within the reference $X$ at all scales. For discrete shapes, a practical algorithm to implement the discrete reconstruction opening is based on the conditional dilation of $M$ by $B$ within $X$ :

$$
\delta_{B}(M \mid X) \triangleq(M \oplus B) \cap X
$$

where $B$ is the unit-radius discrete disk associated with the selected connectivity of the rectangular grid; i.e., a 5-pixel rhombus or a 9-pixel square depending on whether we have 4- or 8-neighbor connectivity, respectively. By iterating this conditional dilation we can obtain in the limit the whole marked component(s) $X_{j}$, i.e. the conditional reconstruction opening

$$
\varrho_{B}^{-}(M \mid X)=\lim _{k \rightarrow \infty} Y_{k}, \quad Y_{k}=\delta_{B}\left(Y_{k-1} \mid X\right), \quad Y_{0}=M .
$$

An example is shown in Fig. 4.

Replacing the binary with graylevel images, the set dilation with function dilation, and $\cap$ with $\wedge$ yields the conditional graylevel reconstruction opening of a digital graylevel image $f$ from a marker image $m$ :

$$
\varrho_{B}^{-}(m \mid f)=\lim _{k \rightarrow \infty} g_{k}, \quad g_{k}=\left(g_{k-1} \oplus B\right) \wedge f, \quad g_{0}=m \leq f .
$$

This reconstructs the bright components of the reference image $f$ that contain the marker $m$. For example, as shown in Fig. 2, the results of any prior image smoothing, like the radial opening of 


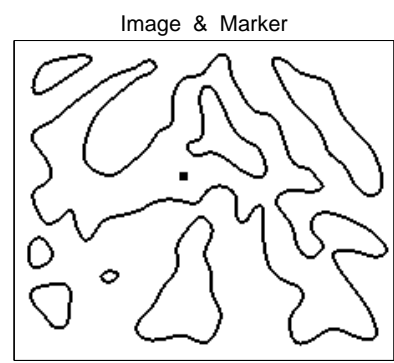

(a)

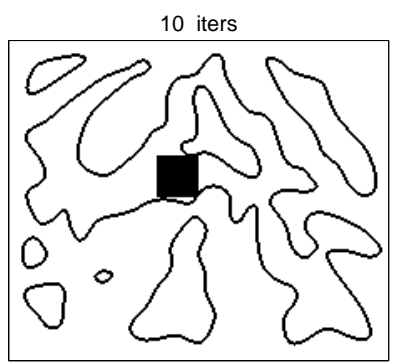

(b)

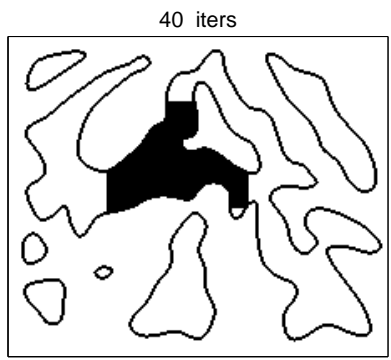

(c)

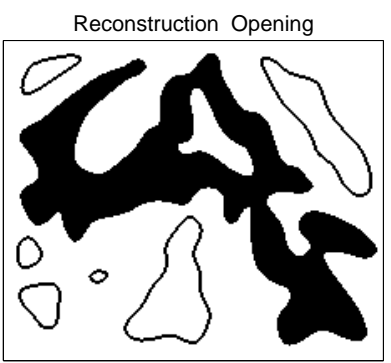

(d)

Figure 4: (a) Original binary image $(192 \times 228$ pixels $)$ and a square marker within the largest component. The next three images show sample iterations of the conditional dilation of the marker with a $3 \times 3$-pixel square structuring element. (b) 10 iterations. (c) 40 iterations. (d) Reconstruction opening, reached after 128 iterations.

Fig. 2(b), can be treated as a marker which is subsequently conditionally dilated under the original reference image to reconstruct Fig. 2(c) exactly those bright image components whose parts have remained after the first operation.

There is a large variety of reconstruction openings depending on the choice of the marker. Two useful cases are (i) size-based markers chosen as the Minkowski erosion $m=f \ominus r B$ of the reference image $f$ by a disk of radius $r$, and (ii) contrast-based markers chosen as the difference $m(x)=f(x)-h$ of a constant $h>0$ from the image. In both cases, the marker is a function of the reference signal. There are also dual definitions for markers suitable for reconstruction closings, where the marker should be larger than the reference.

Reconstruction of the dark image components hit by some marker is accomplished by the dual filter, the reconstruction closing

$$
\varrho_{B}^{+}(m \mid f)=\lim _{k \rightarrow \infty} g_{k}, \quad g_{k}=\left(g_{k-1} \ominus B\right) \vee f, \quad g_{0}=m \geq f
$$

Examples of graylevel reconstruction filterings are shown in Fig. 5 for a 1D signal and in Fig. 2(c) for a $2 \mathrm{D}$ image. Openings and closings by reconstruction have proven to be extremely useful for image simplification because they can suppress small features and keep only large-scale objects without any smoothing of their boundaries.

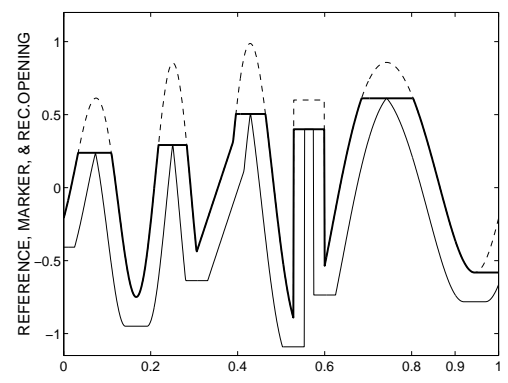

(a)

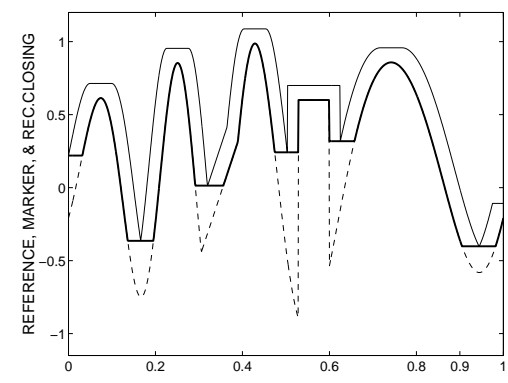

(b)

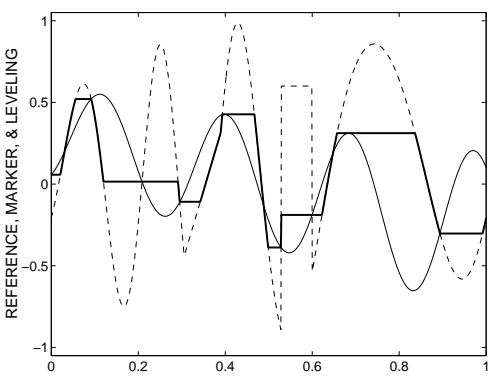

(c)

Figure 5: (a) Reconstruction Opening, (b) Reconstruction Closing and (c) Leveling of a 1D signal. Each subfigure shows the reference signal $f$ (dash line), the marker (thin solid line), and the filter output (thick solid line). In (a) the marker was an erosion of $f$ minus a constant, and hence the filter is a reconstruction opening. In (b) the marker was a dilation of $f$ plus a constant, and hence the filter is a reconstruction closing. In (c) the marker was a Gaussian convolution of $f$ and the filter's output is a leveling. 


\subsubsection{Examples of Order Projections}

Next we mention some examples of order projections, i.e. increasing and idempotent lattice operators, that are neither anti-extensive (i.e. openings) nor extensive (i.e. closings).

Alternating Sequential Filters: A useful combination of openings and closings involves cascading pairs of openings and closings $\beta_{t} \alpha_{t}$ at multiple scales $t=1, \ldots, r$. A simple choice for the multiscale openings and closings is to have Minkowski flat openings $\alpha_{t}(f)=f \circ t B$ and closings $\beta_{t}(f)=f \bullet t B$ of image signals $f$ by multiscale convex sets $t B=\{t b: b \in B\}$ : This generates a class of efficient nonlinear smoothing filters

$$
\psi_{\text {asf }}(f)=\beta_{r} \alpha_{r} \ldots \beta_{2} \alpha_{2} \beta_{1} \alpha_{1}(f)
$$

called alternating sequential filters (ASF), which smooth progressively from the smallest scale possible up to a maximum scale $r$ and have a broad range of applications (Serra, 1988). Their optimal design was studied in Schonfeld and Goutsias (1991). Figure 1(c) shows a single iteration of an ASF.

Further, the Minkowski openings-closings in an ASF can be replaced by other types of lattice openings-closings. Examples of such simple generalized ASFs include replacing each open-closing pair with radial or reconstruction or area open-closings.

Levelings: Despite their many applications, reconstruction openings and closings have as disadvantage the property that they are not self-dual operators; hence, they treat the image and its background asymmetrically. A connected operator that unifies both of them and possesses selfduality is the leveling (Meyer and Maragos, 2000). Levelings are nonlinear 'object-oriented' filters that simplify a reference image $f$ through a simultaneous use of locally expanding and shrinking an initial seed image, called the marker $m$, and globally constraining of the marker evolution by the reference image. Specifically, iterations of the image operator $\phi(m \mid f)=\left(\delta_{B}(m) \wedge f\right) \vee \varepsilon_{B}(m)$, where $\delta_{B}(\cdot)$ (resp. $\left.\varepsilon_{B}(\cdot)\right)$ is a dilation (resp. erosion) by the unit-radius discrete disk $B$ of the grid, yield in the limit the conditional leveling of $f$ w.r.t. $m$ :

$$
\varrho_{B}(m \mid f)=\lim _{k \rightarrow \infty} g_{k}, \quad g_{k}=\left(\delta_{B}\left(g_{k-1}\right) \wedge f\right) \vee \varepsilon_{B}\left(g_{k-1}\right), \quad g_{0}=m
$$

In contrast to the reconstruction opening (closing) where the marker $m$ is smaller (greater) than $f$, the marker for a general leveling may have an arbitrary ordering w.r.t. the reference signal. See Fig. 5(c) for a 1D example and Fig. 1(d) for a 2D example. The leveling reduces to being a reconstruction opening (closing) over regions where the marker is smaller (greater) than the reference image. Actually, it can be shown that the leveling can be obtained as a cascade of a reconstruction opening and a reconstruction closing.

If the marker is self-dual, then the leveling is a self-dual filter and hence treats symmetrically the bright and dark objects in the image. Thus, the leveling may be called a self-dual reconstruction filter. It simplifies both the original image and its background by completely eliminating smaller objects inside which the marker cannot fit. The reference image plays the role of a global constraint. In general, levelings have many interesting multiscale properties (Meyer and Maragos, 2000) and have proven to be very useful for image simplification toward segmentation because they can suppress small-scale noise or small features and keep only large-scale objects with exact preservation of their boundaries.

\section{Minimax Algebra and Image Operators on Complete Weighted Lattices}

This section extends the lattice theory of mathematical morphology from the combined viewpoints of minimax algebra and translation-invariant systems by introducing a nonlinear signal algebra that 
has both a sup/inf signal superposition structure and two scalar semigroup 'multiplication'-type operations ( $\star$ and its dual $\star^{\prime}$ ) that distribute over sup and inf, respectively. On the set of scalars, this generalized algebraic structure is called 'clodum' (complete lattice-ordered double monoid) and combines ideas from both lattice and minimax algebra. We name the resulting nonlinear spaces complete weighted lattices. They combine the sup-inf lattice structure with a scalar semi-ring arithmetic that possesses generalized 'additions' and $\star$-'multiplications'. Their role for analyzing lattice operators with sup- $\star$ arithmetic is conceptually similar to the role played by linear (sumproduct) vector spaces for linear operators.

This generalized signal algebra unifies four main cases where we have complete weighted lattices: (1) Max-Plus mathematical morphology: classic Minkowski translation-invariant operators, which are nonlinear convolutions of the max-plus type. (2) Max-Product mathematical morphology: Lattice extensions of Minkowski dilations and erosions that are supremal and infimal convolutions with multiplicative structuring elements. (3) Fuzzy image operations and convolutions, where $\star\left(\star^{\prime}\right)$ becomes a fuzzy intersection (union). (4) Binary translation-invariant mathematical morphology: this can be seen as a special case of (1) or (3).

Further, we show that any translation-invariant operator that distributes over the main operations of this nonlinear space, can be represented via generalized sup- $\star$ convolutions. In the finite-dimensional case, such operators admit a matrix representation, i.e. become equivalent to a generalized max- $\star$ or its dual min- $\star^{\prime}$ 'product' of the operator's matrix with the input vectors.

\subsection{Lattice-Ordered Monoids}

The material in Section 4.1 interprets some algebraic structures in minimax algebra (CuninghameGreen, 1979) by using concepts from lattice theory, specifically from lattice-ordered groups (Birkhoff, 1967).

A poset, lattice, or semilattice $\mathcal{L}$ is often endowed with additional structure of the group type. Namely, $\mathcal{L}$ may have an additional binary operation, called symbolically the 'multiplication' $\star$, under which $(\mathcal{L}, \star)$ is any of the following:

Semigroup if $\star$ is associative.

Monoid if $\star$ is associative and has identity element.

Group if $\star$ is associative, has identity, and each element has an inverse.

In addition, if $\star$ is also commutative, we obtain a commutative semigroup/monoid/group. Henceforth, we shall deal only with commutative (semi)group operations $\star$.

A lattice-ordered group is an algebra $(\mathcal{L}, \vee, \wedge, \star)$ in which $(\mathcal{L}, \vee, \wedge)$ is a lattice, $(\mathcal{L}, \star)$ is a group, and the group 'multiplication' is increasing. It follows that any group translation $X \mapsto A \star X$ is a lattice automorphism.

An algebra $(\mathcal{M}, \vee, \star)$ is called a semilattice-ordered monoid if $\mathcal{M}$ is a sup-semilattice under $\vee$, monoid under $\star$, and $\star$ distributes over $\vee$ :

$$
A \star(X \vee Y)=(A \star X) \vee(A \star Y)
$$

for all $A, B, X, Y \in \mathcal{M}$. If $\mathcal{M}$ also has an infimum $\wedge$ that (together with $\vee$ ) makes it a lattice, then $(\mathcal{M}, \vee, \wedge, \star)$ is called a lattice-ordered monoid. Suppose now that $\mathcal{M}$ is also a monoid under a 'dual multiplication' operation $\star^{\prime}$ that distributes over infimum:

$$
A \star^{\prime}(X \wedge Y)=\left(A \star^{\prime} X\right) \wedge\left(A \star^{\prime} Y\right)
$$

Now $\mathcal{M}$ has four binary operations. We call the resulting algebra $\left(\mathcal{M}, \vee, \wedge, \star, \star^{\prime}\right)$ a lattice-ordered double monoid. To the above definitions we add the word complete if $\mathcal{M}$ is a complete lattice and the distributivities involved are infinite. For algebraic structures similar to the above definitions alternative names ${ }^{12}$ have been used in previous works on minimax algebra and discrete-event control

\footnotetext{
${ }^{12}$ In minimax algebra (Cuninghame-Green, 1979) a semilattice is called a band. Further, a semilattice-ordered semigroup is called a belt, and a lattice-ordered double semigroup is called a belt with duality. A belt $(\mathcal{B}, \vee, \star)$ with an identity element for the semigroup operation $\star$ and with an element $\zeta$ that is both the least element w.r.t. $\leq$ and also a null, i.e. $a \vee \zeta=a$ and $a \star \zeta=\zeta, \forall a \in \mathcal{B}$, is called a dioid in Cohen et al. (1989).
} 
systems.

In any lattice-ordered double monoid the distributivity of $\star$ over $\vee$ and of $\star^{\prime}$ over $\wedge$ imply that both $\star$ and $\star^{\prime}$ are increasing; i.e.,

$$
\begin{aligned}
& X \leq Y \quad \Longrightarrow \quad A \star X \leq A \star Y \\
& X \leq Y \quad \Longrightarrow A \star^{\prime} X \leq A \star^{\prime} Y
\end{aligned}
$$

These properties imply in turn that

$$
\begin{aligned}
& A \star(X \wedge Y) \leq(A \star X) \wedge(A \star Y) \\
& A \star^{\prime}(X \vee Y) \geq\left(A \star^{\prime} X\right) \vee\left(A \star^{\prime} Y\right)
\end{aligned}
$$

If $\star=\star^{\prime}$, we have a self-dual 'multiplication'. This always happens if $(\mathcal{M}, \star)$ is a group; in this case we obtain a lattice-ordered group, and the inequalities (94) become equalities.

\subsection{Clodum: An Algebraic Structure for Weighted Lattice Arithmetic}

We henceforth assume that all vector elements or signals involved in the description of the operators and systems examined herein take their values from a set $\mathcal{C}$ of scalars, which in general will be a subset of the set $\overline{\mathbb{R}}=\mathbb{R} \cup\{-\infty, \infty\}$ of extended real numbers. Under the standard real number ordering $\leq, \mathcal{C}$ is a chain, and $\bigvee$ and $\bigwedge$ become the standard supremum and infimum on the reals. We assume that $\mathcal{C}$ is universally bounded, i.e., contains its least $\mathcal{C}_{\mathrm{O}} \triangleq \wedge \mathcal{C}$ and greatest element $\mathcal{C}_{\mathrm{I}} \triangleq \bigvee \mathcal{C}$. For the unified nonlinear signal processing algebra examined herein we need to equip $\mathcal{C}$ with four binary operations:

(A). A generalized 'addition' under which $\mathcal{C}$ becomes a complete sup-semilattice. We shall henceforth fix this 'addition' to be the standard supremum $\vee$ on $\overline{\mathbb{R}}$.

$\left(\mathrm{A}^{\prime}\right)$. A dual 'addition' which makes $\mathcal{C}$ a complete inf-semilattice and is related to the generalized 'addition' via the absorption law L4 of Table 1 . The standard infimum $\wedge$ on $\overline{\mathbb{R}}$ will henceforth be this dual 'addition'.

$(\mathrm{M})$. A commutative generalized 'multiplication' $\star$ under which: (i) $\mathcal{C}$ is a monoid with a ('unit') identity element $\mathcal{C}_{\text {id }}$,

$$
a \star \mathcal{C}_{\mathrm{id}}=a, \quad \forall a \in \mathcal{C},
$$

a ('zero') null element $\mathcal{C}_{\mathrm{O}}$,

$$
a \star \mathcal{C}_{\mathrm{O}}=\mathcal{C}_{\mathrm{O}}, \quad \forall a \in \mathcal{C},
$$

and (ii) $\star$ is a scalar dilation, i.e., distributes over any supremum

$$
a \star\left(\bigvee_{i \in J} x_{i}\right)=\bigvee_{i \in J}\left(a \star x_{i}\right)
$$

for any (possibly infinite) index set $J$. So far $(\mathcal{C}, \vee, \wedge, \star)$, i.e. the value set equipped only with three operations, is a dioid, which is a structure defined in Cohen et al. (1989). Also, note that the properties (96),(97) imply that

$$
a \star \mathcal{C}_{\mathrm{I}}=\mathcal{C}_{\mathrm{I}}, \quad \forall a \neq \mathcal{C}_{\mathrm{O}} .
$$

$\left(\mathrm{M}^{\prime}\right)$. A commutative dual 'multiplication' $\star^{\prime}$ under which: (i) $\mathcal{C}$ is a monoid with an identity $\mathcal{C}_{\text {id }}^{\prime}$

$$
a \star^{\prime} \mathcal{C}_{\text {id }}^{\prime}=a, \quad \forall a \in \mathcal{C},
$$

a null element $\mathcal{C}_{\mathrm{I}}$

$$
a \star^{\prime} \mathcal{C}_{\mathrm{I}}=\mathcal{C}_{\mathrm{I}}, \quad \forall a \in \mathcal{C},
$$

and (ii) $\star^{\prime}$ is a scalar erosion, i.e., distributes over any infimum:

$$
a \star^{\prime}\left(\bigwedge_{i \in J} x_{i}\right)=\bigwedge_{i \in J}\left(a \star^{\prime} x_{i}\right)
$$


Also, note that the properties (100),(101) imply that

$$
a \star^{\prime} \mathcal{C}_{\mathrm{O}}=\mathcal{C}_{\mathrm{O}}, \quad \forall a \neq \mathcal{C}_{\mathrm{I}} .
$$

We group the above requirements into the following sets of conditions:

$(\mathrm{C} 1) .(\mathcal{C}, \vee, \wedge)$ is a complete infinitely-distributive lattice.

$(\mathrm{C} 2) .(\mathcal{C}, \star)$ is a commutative monoid, and $\star$ is a dilation.

(C3). $\left(\mathcal{C}, \star^{\prime}\right)$ is a commutative monoid, and $\star^{\prime}$ is an erosion.

Under the above assumptions $\left(\mathcal{C}, \vee, \wedge, \star, \star^{\prime}\right)$ becomes a commutative complete lattice-ordered double monoid, in short clodum. This will be the most general and minimally required algebraic structure we consider for the set of scalars. We avoid degenerate cases by henceforth assuming that each 'addition' is different from its corresponding 'multiplication', i.e., $\vee \neq \star$ and $\wedge \neq \star$ '. However, $\star$ may be the same as $\star^{\prime}$, in which case we have a self-dual 'multiplication'.

In some cases we may have some additional algebraic structure in $\mathcal{C}$. This occurs if we assume that $\mathcal{C}=\mathcal{C}_{\mathrm{G}} \cup\left\{\mathcal{C}_{\mathrm{O}}, \mathcal{C}_{\mathrm{I}}\right\}$ where $\left(\mathcal{C}_{\mathrm{G}}, \star\right)$ is a commutative group. Then, for each element $a \in \mathcal{C}_{\mathrm{G}}$ there exists its 'multiplicative' inverse $a^{-1}$ such that $a \star a^{-1}=\mathcal{C}_{\text {id }}$. Further, $\left(\mathcal{C}_{\mathrm{G}}, \vee, \wedge, \star, \star\right)$ is a latticeordered group with self-dual 'multiplication'. The 'multiplication' $\star$ and its self-dual $\star^{\prime}$ (which coincide over $\mathcal{C}_{\mathrm{G}}$ ) can be extended over the entire $\mathcal{C}$ by adding the rules in (96) and (100) involving the null elements. The resulting richer structure $(\mathcal{C}, \vee, \wedge, \star, \star)$ is called a bounded lattice-ordered group in Cuninghame-Green (1979), in short blog.

A clodum $\mathcal{C}$ is called self-conjugate if it has a negation, i.e. an involutive dual automorphism that maps each element $a$ to its conjugate element $a^{*}$ such that

$$
\begin{aligned}
& \left(\bigvee_{i} a_{i}\right)^{*}=\bigwedge_{i} a_{i}{ }^{*} \\
& \left(\bigwedge_{i} b_{i}\right)^{*}=\bigvee_{i} b_{i}{ }^{*} \\
& (a \star b)^{*}=a^{*} \star^{\prime} b^{*}
\end{aligned}
$$

The first two above properties are generalization of De Morgan's laws in Boolean algebras. As for the 'multiplication' operations, we assume that the negation also distributes over any (possibly infinite) suprema and infima. If $\mathcal{C}$ is a blog, then it becomes self-conjugate by setting

$$
a^{*}=\left\{\begin{array}{lll}
a^{-1} & \text { if } & \mathcal{C}_{\mathrm{O}}<a<\mathcal{C}_{\mathrm{I}} \\
\mathcal{C}_{\mathrm{I}} & \text { if } & a=\mathcal{C}_{\mathrm{O}} \\
\mathcal{C}_{\mathrm{O}} & \text { if } & a=\mathcal{C}_{\mathrm{I}}
\end{array}\right.
$$

\subsection{Nonlinear Spaces based on Clodums: Complete Weighted Lattices}

\subsubsection{General Algebraic Structure}

We are interested in creating nonlinear spaces whose algebraic structure will resemble that of traditional linear spaces, like for example the spaces $\mathbb{R}^{n}$ of vectors or the spaces $\ell^{p}\left(\mathbb{Z}^{m}, \mathbb{R}\right)$ of signals with finite norm, but whose vector/signal addition and scalar multiplication will be replaced by the lattice supremum and infimum operations and the scalar addition and multiplication in the field of scalars supporting a linear space will be replaced by the scalar arithmetic of a clodum.

Consider a nonempty collection $\mathcal{W}$ of mathematical objects, which will be our space; examples of such objects include the vectors in $\overline{\mathbb{R}}^{n}$ or signals in $\operatorname{Fun}(E, \overline{\mathbb{R}})$. Thus, we shall symbolically refer to the space elements as 'vectors/signals', although they may be arbitrary objects. Also, consider a clodum $\left(\mathcal{C}, \vee, \wedge, \star, \star^{\prime}\right)$ of scalars. We define two operations among vectors/signals $F, G$ in $\mathcal{W}$ : their supremum $F \vee G: \mathcal{W}^{2} \rightarrow \mathcal{W}$ and infimum $F \wedge G: \mathcal{W}^{2} \rightarrow \mathcal{W}$, which we denote using the same supremum symbol $(\vee)$ and infimum symbol $(\wedge)$ as in the clodum, hoping that the differences will be clear to the reader from the context. Further, we define two operations among any vector/signal $F$ in $\mathcal{W}$ and any scalar in $c$ in $\mathcal{C}$ : a scalar 'multiplication' $c \star F:(\mathcal{C}, \mathcal{W}) \rightarrow \mathcal{W}$ and a scalar dual multiplication' $c \star^{\prime} F:(\mathcal{C}, \mathcal{W}) \rightarrow \mathcal{W}$; again, we denote these scalar 'multiplications' of vectors/signals by using the same symbols as in the clodum. Now, we define $\mathcal{W}$ to be a weighted lattice (WL) space over a clodum $\left(\mathcal{C}, \vee, \wedge, \star, \star^{\prime}\right)$ of scalars if for all $F, G, H \in \mathcal{W}$ and $a, b \in \mathcal{C}$ the 
following axioms hold:

(WL-A1). $F \vee G \in \mathcal{W}$ and $F \wedge G \in \mathcal{W}$,

(WL-A2). $F \vee G=G \vee F$ and $F \wedge G=G \wedge F$,

(WL-A3). $F \vee(G \vee H)=(F \vee G) \vee H$ and $F \wedge(G \wedge H)=(F \wedge G) \wedge H$, (Associativity of sup/inf).

(WL-A4). $F \vee O=F$ and $F \wedge I=F$,

(WL-A5). $F \vee F=F$ and $F \wedge F=F$,

(WL-A6). $F \vee(F \wedge G)=F$ and $F \wedge(F \vee G)=F$,

(Existence of 'addition' identities).

(Idempotence of sup/inf).

(Absorption between sup/inf).

(WL-A7). $F \vee(G \wedge H)=(F \vee G) \wedge(F \vee H)$ and $F \wedge(G \vee H)=(F \wedge G) \vee(F \wedge H)$, (Distributivity of $\sup /$ inf).

(WL-SM1). $a \star F \in \mathcal{W}$ and $a \star^{\prime} F \in \mathcal{W}$,

(Closure under scalar 'multiplications').

(WL-SM2). $a \star(b \star F)=(a \star b) \star F$ and $a \star^{\prime}\left(b \star^{\prime} F\right)=\left(a \star^{\prime} b\right) \star^{\prime} F$,

(Associativity of scalar 'multiplications').

(WL-SM3). $\mathcal{C}_{\mathrm{id}} \star F=F$ and $\mathcal{C}_{\text {id }}^{\prime} \star^{\prime} F=F$,

(WL-SM4). $\mathcal{C}_{\mathrm{O}} \star F=O$ and $\mathcal{C}_{\mathrm{I}} \star^{\prime} F=I$,

('Multiplication' with scalar identities).

(WL-A\&SM1). $a \star(F \vee G)=a \star F \vee a \star G$ and $a \star^{\prime}(F \wedge G)=a \star^{\prime} F \wedge a \star^{\prime} G$,

A\&SM 1)

(WL-A\&SM2). $(a \vee b) \star F=a \star F \vee b \star F$ and $(a \wedge b) \star^{\prime} F=a \star^{\prime} F \wedge b \star^{\prime} F$, (Distributivity A\&SM 2)

We observe the following:

(1) The six axioms from (WL-A1) until (WL-A6) make $(\mathcal{W}, \vee, \wedge)$ a lattice with a least element $(O)$ and a greatest element $(I)$.

(2) Axiom (WL-A7) makes this lattice distributive.

(3) The above axioms of a weighted lattice (WL) space bear a striking similarity with those of a linear space in Section 2.1.1. For example, compare the 'addition' axioms (WL-A1) until (WL-A4) with the axioms(A1)-(A4) of linear spaces. Similarly, compare the 'multiplication' axioms from (WL-SM1) until (WL-SM4) with the axioms (SM1)-(SM4) of linear spaces. Finally, compare the combined 'addition' and 'multiplication' axioms from (WL-A\&SM1) until (WL-A\&SM2) with the axioms (A\&SM1)-(A\&SM2) of linear spaces. One difference is that the vector/signal addition $(+)$ of linear spaces is now replaced by two dual superpositions, the lattice supremum $(V)$ and infimum $(\wedge)$; further, the scalar multiplication $(\times)$ of linear spaces is now replaced by two operations $\star$ and $\star^{\prime}$ that are dual to each other. Only one major property of the linear spaces is missing from the weighted lattices: the existence of 'additive inverses'; i.e., the supremum and infimum operations do not have inverses.

We shall define the weighted lattice $\mathcal{W}$ over the clodum $\mathcal{C}$ to be a complete weighted lattice (CWL) space if all the following hold:

(i) $\mathcal{W}$ is closed under any, possibly infinite, suprema and infima.

(ii) Its distributivities between supremum and infimum are of the infinite type.

(iii) The distributivities between the scalar operations $\star$ or $\star^{\prime}$ and the supremum or infimum are of the infinite type.

Note that, a clodum is by itself a scalar complete weighted lattice over itself.

A subset $\mathcal{X}$ of a (complete) weighted lattice $\mathcal{W}$ over a scalar clodum $\mathcal{C}$ is called a (complete) weighted sublattice if it is itself a (complete) weighted lattice over $\mathcal{C}$, or equivalently if it is (completely ${ }^{13}$ ) closed under the original lattice supremum and infimum as well as by the two scalar multiplications.

\footnotetext{
${ }^{13}$ When we call a weighted lattice 'completely closed' under the supremum and infimum as well as by the two scalar
ultiplications, we mean that it is closed under arbitrary suprema and infima and all its distributivities are infinite,

${ }^{13}$ When we call a weighted lattice 'completely closed' under the supremum and infimum as well as by the two scalar
multiplications, we mean that it is closed under arbitrary suprema and infima and all its distributivities are infinite, both between supremum and infimum, as well as between the scalar operations $\star$ or $\star^{\prime}$ and the supremum or infimum respectively.
} 


\subsubsection{Sup/Inf Span, Independence, Basis, Dimension}

Consider a subset $\mathcal{A}$ of a complete weighted lattice $\mathcal{W}$ over a clodum $\mathcal{C}$. A space element $F$ is called a sup- $\star$ combination of points in $\mathcal{A}$ if there exists an indexed set of space elements $\left\{F_{i}\right\}$ in $\mathcal{A}$ and a corresponding set of scalars $\left\{a_{i}\right\}$ in $\mathcal{C}$ such that

$$
F=\bigvee_{i} a_{i} \star F_{i}
$$

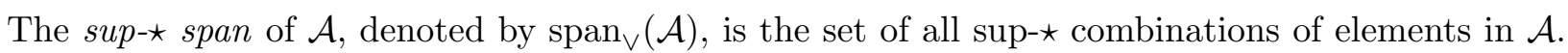
If $\mathcal{A}=\varnothing$, then $\operatorname{span}_{\vee}(\mathcal{A})=\{O\}$. Dually, a space element $G$ is called an inf- $\star^{\prime}$ combination of points in $\mathcal{A}$ if there exists an indexed set of elements $\left\{G_{i}\right\}$ in $\mathcal{A}$ and a corresponding set of scalars $\left\{b_{i}\right\}$ in $\mathcal{C}$ such that

$$
G=\bigwedge_{i} b_{i} \star^{\prime} G_{i},
$$

The inf- $\star^{\prime}$ span of $\mathcal{A}$, denoted by $\operatorname{span}_{\wedge}(\mathcal{A})$, is the set of all inf- $-\star^{\prime}$ combinations of elements in $\mathcal{A}$. If $\mathcal{A}=\varnothing$, by convention we set $\operatorname{span}_{\wedge}(\mathcal{A})=\{I\}$.

If the above sup- $\star$ and inf- $\star^{\prime}$ combination are based on a finite set of space elements, we shall

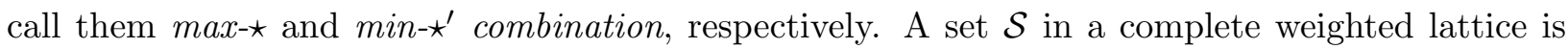
called max- $\star$ independent (resp. min- $\star^{\prime}$ independent) if each point $f \in \mathcal{S}$ is not a max- $\star$ (resp.

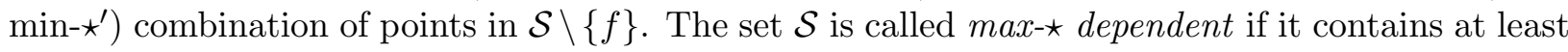
one element $F$ that is a max- $\star$ combination of points in $S \backslash\{F\}$. Dually for the min- $\star^{\prime}$ dependence.

Recall the definition in linear spaces of a Hamel basis as a subset of the space that is linearly independent and its linear span makes up all the space. In the nonlinear spaces under discussion, a subset $\mathcal{B}$ of a complete weighted lattice $\mathcal{W}$ is called an upper basis for the space if each element $F$ of the space can be represented as a sup- $\star$ combination of (i.e. supremum of $\star$-translated) basis elements:

$$
F=\bigvee_{i} c_{i} \star B_{i}, \quad B_{i} \in \mathcal{B}
$$

Dually, a subset $\mathcal{B}^{\prime}$ of $\mathcal{W}$ is called a lower basis for the space if each element of the space can be represented as a min- $\star$ combination of (i.e. infimum of $\star^{\prime}$-translated) basis elements:

$$
F=\bigwedge_{i} d_{i} \star^{\prime} B_{i}^{\prime}, \quad B_{i}^{\prime} \in \mathcal{B}^{\prime}
$$

If the space $\mathcal{W}$ is self-conjugate, then (107) implies that

$$
F^{*}=\bigwedge_{i} c_{i}^{*} \star^{\prime} B_{i}^{*}
$$

Thus, if the space possesses an upper basis, it will possess a lower basis too. We conjecture that the upper and lower bases of a complete weighted lattice have the same cardinality. This cardinality is called the dimension of $\mathcal{W}$. If this is finite, the space is called finite-dimensional; otherwise, it is called infinite-dimensional. Examples of an upper and a lower basis are mentioned in Section 4.4.1 and Section 4.7 for signal and vector spaces respectively; in the first case the basis is infinite-dimensional, whereas the second case is finite-dimensional.

\subsubsection{Complete Weighted Lattices of Functions}

In this chapter we are primarily interested in working on complete weighted lattice ${ }^{14}$ (CWL) spaces of signals and vectors. Thus, the underlying set of our CWL space is a function space $\mathcal{W}=$

\footnotetext{
${ }^{14}$ Our definition of complete weighted lattices (CWL) is general and can also be applied to collections of objects that are different than vectors or signals. It is only because this chapter deals with signal operators that we have focused on CWL signal or vector spaces and have defined the 'additions' and scalar 'multiplications' by extending pointwise the corresponding scalar operations of the clodum to functions.
} 
Fun $(E, \mathcal{C})$ where $E$ is an arbitrary nonempty set playing the role of the domain of our functions. The values of these functions are in a set $\mathcal{C}$ of scalars, which for our purposes will generally be a subset of $\overline{\mathbb{R}}$. For the value set $\mathcal{C}$ we assume that it is a clodum equipped with four binary operations, a supremum $\vee$, an infimum $\wedge$, a scalar 'multiplication' $\star$ and a dual scalar 'multiplication' $\star^{\prime}$ satisfying the following properties:

$(1) .(\mathcal{C}, \vee, \wedge)$ is a complete infinitely distributive lattice.

(2). $(\mathcal{C}, \star)$ is a commutative monoid, and $\star$ distributes over any supremum $\vee$.

(3). $\left(\mathcal{C}, \star^{\prime}\right)$ is a commutative monoid, and $\star^{\prime}$ distributes over any infimum $\wedge$.

Then, we extend pointwise the supremum, infimum and scalar multiplications of $\mathcal{C}$ to the functions: for all $F, G \in \mathcal{W}$ and $a \in \mathcal{C}$

$$
\begin{aligned}
& (F \vee G)(x) \triangleq F(x) \vee G(x), \quad x \in E \\
& (F \wedge G)(x) \triangleq F(x) \wedge G(x), \quad x \in E \\
& (a \star F)(x) \triangleq a \star F(x), \quad x \in E \\
& \left(a \star^{\prime} F\right)(x) \triangleq a \star^{\prime} F(x), \quad x \in E
\end{aligned}
$$

Assuming that the function collection $\mathcal{W}$ is closed under the above four operations, then $\mathcal{W}$ becomes a complete infinitely distributive lattice that inherits many properties from the lattice structure of $\mathcal{C}$. The least $(O)$ and greatest $(I)$ elements of $\mathcal{W}$ are the functions

$$
O(x)=\mathcal{C}_{\mathrm{O}}, \quad I(x)=\mathcal{C}_{\mathrm{I}}, \quad \forall x \in E .
$$

Further, the scalar operations $\star$ and $\star^{\prime}$, extended pointwise to functions, distribute over any suprema and infima, respectively. Thus, the function $\operatorname{space} \operatorname{Fun}(E, \mathcal{C})$ is by construction a complete weighted lattice of functions over the clodum $\mathcal{C}$. The collection of all its properties creates a rich algebraic structure.

If the clodum $\mathcal{C}$ is self-conjugate, i.e. has a negation $(\cdot)^{*}$ satisfying (103), then we can extend the conjugation to elements $F$ of the space $\mathcal{W}$ pointwise:

$$
F^{*}(x) \triangleq(F(x))^{*}, \quad x \in E
$$

In such a case we talk about a self-conjugate complete weighted lattice.

Example 9 (Complete Weighted Lattice spaces):

(a) CWL Vector spaces: $E=\{1,2, \ldots, n\}, \mathcal{C}=\overline{\mathbb{R}}$. These are essentially complete minimax ${ }^{15}$ vector spaces. See Section 4.7.

(b) CWL Signal spaces: $E=\mathbb{R}^{m} E=\mathbb{Z}^{m}, \mathcal{C}=\overline{\mathbb{R}}$. See Section 4.4.1.

\subsection{Image Operators on Complete Weighted Lattices}

\subsubsection{Image Space, Impulse Representations and Vertical Translations}

Based on our previous discussion, the collection $\mathcal{S}=\operatorname{Fun}(E, \mathcal{C})$ of functions with values in the clodum $\left(\mathcal{C}, \vee, \wedge, \star, \star^{\prime}\right)$ becomes a complete weighted lattice (CWL) function space, where the four operations of $\mathcal{C}$ are extended pointwise to functions. Of main importance is the case $E=\mathbb{R}^{2}$ or $E=\mathbb{Z}^{2}$, where $\mathcal{S}$ becomes the set of all image signals defined on the continuous or discrete image plane and taking scalar values in $\mathcal{C}$; the multidimensional domain $\mathbb{E}^{m}$ is also a straightforward extension. But $E$ could also be a finite index set, e.g. $E=\{1,2, \ldots, n\}$ for matrix-based image processing or the set of vertices of a graph in cases of images defined on a graph.

\footnotetext{
${ }^{15} \mathrm{By}$ 'minimax vector spaces' we mean the finite-dimensional nonlinear vector spaces of minimax algebra that are equipped with max-plus arithmetic or its dual and corresponding nonlinear matrix operations (Cuninghame-Green, 1979).
} 
Viewed as a lattice, $\mathcal{S}$ possesses semi-atoms $q_{y, v}$ and dual semi-atoms $q_{y, v}^{\prime}$ which are the following elementary pulse functions

$$
q_{y, v}(x) \triangleq\left\{\begin{array}{ll}
v, & x=y \\
\mathcal{C}_{\mathrm{O}}, & x \neq y
\end{array}, \quad q_{y, v}^{\prime}(x) \triangleq \begin{cases}v, & x=y \\
\mathcal{C}_{\mathrm{I}}, & x \neq y\end{cases}\right.
$$

Further, since $\mathcal{S}$ also has a monoid structure, we can consider generalized translations of function values via their ' $\star$-multiplication' by constants $v$, denoted as $\lambda_{v}: a \mapsto a \star v$; we call them vertical translations, in short $V$-translations, since geometrically they affect the function graph in the vertical direction. Similarly we can define dual vertical translations $\lambda_{v}^{\prime}: a \mapsto a \star^{\prime} v$. The scalar mappings $\lambda, \lambda^{\prime}$ can be extended to functions pointwise; we keep the same symbol for both scalar and function operations:

$$
\lambda_{v}(F)(x) \triangleq \lambda_{v}[F(x)]=v \star F(x), \quad \lambda_{v}^{\prime}(F)(x) \triangleq \lambda_{v}^{\prime}[F(x)]=v \star^{\prime} F(x)
$$

Now, the function semi-atoms can be expressed as V-translations of only those whose height equals the identity. Namely, if we define

$$
q_{y}(x) \triangleq\left\{\begin{array}{ll}
\mathcal{C}_{\mathrm{id}}, & x=y \\
\mathcal{C}_{\mathrm{O}}, & x \neq y
\end{array}, \quad q_{y}^{\prime}(x) \triangleq \begin{cases}\mathcal{C}_{\mathrm{id}}^{\prime}, & x=y \\
\mathcal{C}_{\mathrm{I}}, & x \neq y\end{cases}\right.
$$

as the impulse and dual impulse ${ }^{16}$ functions, respectively, then all semi-atoms can be expressed as V-translations of the impulse functions:

$$
q_{y, v}(x)=v \star q_{y}(x), \quad q_{y, v}^{\prime}(x)=v \star^{\prime} q_{y}^{\prime}(x)
$$

Hence, since $\mathcal{S}$ is a semi-atomic lattice, every function $F(x)$ admits a representation as a supremum of V-translated impulses placed at all points of the domain $E$ or as infimum of dual Vtranslated impulses:

$$
F(x)=\bigvee_{y \in E} F(y) \star q_{y}(x)=\bigwedge_{y \in E} F(y) \star^{\prime} q_{y}^{\prime}(x)
$$

Note that the collection of semi-atoms $q_{y, v}$ (resp. dual semi-atoms $q_{y, v}^{\prime}$ ) constitutes a supgenerating (resp. inf-generating) subset of $\mathcal{S}$ viewed as a lattice; see (31). Further, the collection of impulses $q_{y}(x)$ (resp. dual impulses $q_{y}^{\prime}(x)$ ) is an upper (resp. lower) basis for the space $\mathcal{S}$ viewed as a complete weighted lattice. If $E=\mathbb{R}^{m}$ or $\mathbb{Z}^{m}$, then these bases are infinite since the number or required impulses is infinite. However, if $E=\{1,2, \ldots, n\}$ as is the case of vector spaces, then we have finite bases; this case is detailed in Section 4.7 .

\subsubsection{Representation of Dilations and Erosions Invariant Under Vertical Translations}

We are interested in increasing operators on the complete weighted lattice $(\mathrm{CWL})$ space $\mathcal{S}=$ $\operatorname{Fun}(E, \mathcal{C})$ of functions. The previous $\mathrm{V}$-translations $\lambda_{v}: F \mapsto v \star F$ of functions $F$ via 'multiplication' by constants $v$ are increasing operators. Actually they are dilations of the simplest type, which we shall often call elementary function dilations. Their collection ${ }^{17} \mathbb{L}=\left\{\lambda_{v}: v \in \mathcal{C}\right\}$ forms under composition a commutative monoid of function dilations:

$$
\lambda_{a} \lambda_{b}=\lambda_{a \star b}
$$

\footnotetext{
${ }^{16}$ We may also call $q$ and $q^{\prime}$ an upper impulse and a lower impulse, respectively.

${ }^{17}$ In some specialized cases, the need may arise to restrict the vertical translations only by scalars $v$ that are not extreme elements in the complete lattice $\mathcal{C}$, i.e. to not allow $v$ to equal the least element $\mathcal{C}_{\mathrm{O}}$ or greatest element $\mathcal{C}_{\mathrm{I}}$ of $\mathcal{C}$. Such a case may arise when $\mathcal{C}$ is a blog, because then $\mathcal{C}=\mathcal{C}_{\mathrm{G}} \cup\left\{\mathcal{C}_{\mathrm{O}}, \mathcal{C}_{\mathrm{I}}\right\}$ where $\left(\mathcal{C}_{\mathrm{G}}, \star\right)$ is a group; for example, if $\mathcal{C}=\overline{\mathbb{R}}$, then $\mathcal{C}_{\mathrm{G}}=\mathbb{R}$ and $\left(\mathcal{C}_{\mathrm{G}},+\right)$ is the additive group of finite reals. We will leave our discussion general, i.e. without such restrictions, and wherever needed we shall mention any exceptions. Besides, it is always easy to set a few rules and correctly handle the two extreme elements of $\mathcal{C}$ for the clodum arithmetic.
} 
A function mapping $\psi$ is called $V$-translation invariant operator, in short $\mathbb{L}$-operator, if it commutes with any $\mathrm{V}$-translation, i.e., $\psi \lambda_{v}=\lambda_{v} \psi$ for all $v$.

All the above concepts apply as well for function translations via dual 'multiplication'. Each dual V-translation $\lambda_{v}^{\prime}: F \mapsto v \star^{\prime} F$ is an elementary function erosion, and their collection $\mathbb{L}^{\prime}=\left\{\lambda_{v}^{\prime}\right.$ : $v \in \mathcal{C}\}$ is a monoid of function erosions. Namely, we call an operator dual $V$-translation invariant iff it commutes with any such dual $\mathrm{V}$-translation. If $\mathcal{C}$ is a blog, the above two monoids $\mathbb{L}, \mathbb{L}^{\prime}$, after restriction to non-extreme translations $v \in \mathcal{C}_{\mathrm{G}}$, become the same group of automorphisms on the function lattice.

Important examples of increasing operators are the dilations and erosions. The following provides a decomposition of function dilations and erosions on the lattice $\mathcal{S}$ into suprema and infima of scalar dilations and erosions on $\mathcal{C}$, respectively.

Proposition 15 (Decomposition of Adjunctions) (Heijmans and Ronse, 1990).

Let $\mathcal{C}$ be a complete lattice and $E$ an arbitrary nonempty set. The pair $(\varepsilon, \delta)$ is an adjunction on the function lattice $\operatorname{Fun}(E, \mathcal{C})$ iff for every $x, y \in E$ there exists an adjunction $\left(e_{x, y}, d_{y, x}\right)$ on $\mathcal{C}$ such that

$$
\delta(F)(x)=\bigvee_{y \in E} d_{y, x}(F(y)) \quad, \quad \varepsilon(G)(y)=\bigwedge_{x \in E} e_{x, y}(G(x))
$$

for $x, y \in E$ and $F, G \in \operatorname{Fun}(E, \mathcal{C})$.

In the space $\mathcal{S}$, if we consider the impulse functions $q_{y}(x)$ and their duals $q_{y}^{\prime}(x)$ in (115), we can enable the decomposition (119) by defining the scalar dilations to be

$$
d_{y, x}(v)=\delta\left(q_{y, v}\right)(x)=\delta\left(v \star q_{y}\right)(x), \quad x, y \in E, v \in \mathcal{C}
$$

and $e_{x, y}$ to be the adjoint erosion of $d_{y, x}$.

Dually we can define the scalar erosions $e_{x, y}$ from the action of $\varepsilon$ on the dual impulses $q^{\prime}$, i.e.

$$
e_{x, y}(v)=\varepsilon\left(q_{x, v}^{\prime}\right)(y)=\varepsilon\left(v \star^{\prime} q_{x}\right)(y), \quad x, y \in E, v \in \mathcal{C}
$$

and then define the scalar dilations $d_{y, x}$ as adjoints of $e_{x, y}$.

An important outcome from the above discussion is that the output functions from dilation (resp. erosion) operators excited by V-translated impulses are sufficient for the supremal (resp. infimal) representation of the operators. Henceforth we assume that these operators are V-translation invariant. For dilations and erosions this invariance implies that they obey an interesting nonlinear superposition principle which has direct conceptual analogies with the linear superposition obeyed by linear operators. Specifically, we define $\delta$ to be a dilation V-translation invariant (DVI) operator iff

$$
\delta\left(\bigvee_{i \in J} c_{i} \star F_{i}\right)=\bigvee_{i \in J} c_{i} \star \delta\left(F_{i}\right), \quad c_{i} \in \mathcal{C}, F_{i} \in \mathcal{S}
$$

for any index set $J$. We also define $\varepsilon$ to be an erosion V-translation invariant (EVI) operator iff

$$
\varepsilon\left(\bigwedge_{i \in J} c_{i} \star^{\prime} F_{i}\right)=\bigwedge_{i \in J} c_{i} \star^{\prime} \varepsilon\left(F_{i}\right), \quad c_{i} \in \mathcal{C}, F_{i} \in \mathcal{S}
$$

Compare the two above nonlinear superpositions with the linear superposition obeyed by a linear operator $\Gamma$ :

$$
\Gamma\left(\sum_{i \in J} a_{i} \cdot F_{i}\right)=\sum_{i \in J} a_{i} \cdot \Gamma\left(F_{i}\right)
$$

where $J$ is a finite index set, $a_{i}$ are constants from a field (e.g. the set of reals or complex numbers) and $F_{i}$ are field-valued signals from a linear space.

If we assume that our operators are V-translation invariant, then their outputs obtain a simplified structure which is best described by defining next the operator's impulse responses. Given a dilation operator $\delta$, its impulse response map is the mapping $H: E \rightarrow \operatorname{Fun}(E, \mathcal{C})$ defined 
at each $y \in E$ as the output function from $\delta$ when the input is the impulse $q_{y}$. Dually, for an erosion operator $\varepsilon$ we define its dual impulse response map $H^{\prime}$ via its outputs when excited by dual impulses:

$$
H_{y}(x) \triangleq \delta\left(q_{y}\right)(x), \quad H_{y}^{\prime}(x) \triangleq \varepsilon\left(q_{y}^{\prime}\right)(x), \quad x, y \in E
$$

The following gives a unified representation for all V-translation invariant dilations and erosions on a CWL function space.

Theorem 10 (Maragos, 2005a).

Let $\mathcal{S}=\operatorname{Fun}(E, \mathcal{C})$ be a complete weighted lattice of functions over a clodum $\left(\mathcal{C}, \vee, \wedge, \star, \star^{\prime}\right)$. Then:

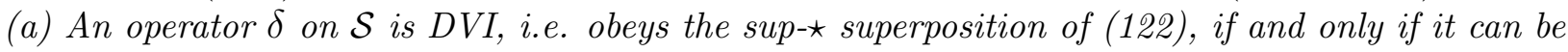
expressed as

$$
\delta(F)(x)=\bigvee_{y \in E} F(y) \star H_{y}(x)
$$

where $H_{y}$ is its impulse response map in (125).

(b) An operator $\varepsilon$ on $\mathcal{S}$ is EVI, i.e. obeys the inf- $\star^{\prime}$ superposition of (123), if and only if it can be expressed as

$$
\varepsilon(F)(x)=\bigwedge_{y \in E} F(y) \star^{\prime} H_{y}^{\prime}(x)
$$

where $H_{y}^{\prime}$ is its dual impulse response map in (125).

Note that, in the case of a signal space $\left(E=\mathbb{E}^{m}\right)$, the operations in (126) and (127) are like adaptive nonlinear convolutions where a dilation (resp. erosion) system's output is obtained as supremum (resp. infimum) of various impulse response signals produced by exciting with impulses at all points and weighted by the input signal values via a group-like $\star$-'multiplication'.

\subsection{Representation of Signal Dilation and Erosion Operators Invariant under Translation Monoids on Complete Weighted Lattices}

In this section we shall work with the complete weighted lattice (CWL) signal space $\mathcal{S}=\operatorname{Fun}(E, \mathcal{C})$ over a clodum $\left(\mathcal{C}, \vee, \wedge, \star, \star^{\prime}\right)$, where the underlying set $\mathcal{S}$ consists of all signals with values from $\mathcal{C} \subseteq \overline{\mathbb{R}}$ and defined on a multidimensional plane-like domain ${ }^{18} E$ that is a subset of the Euclidean domain $\mathbb{R}^{m}$ or its discrete version $\mathbb{Z}^{m}, m=1,2, \ldots$ We shall consider monoids $\mathbb{T}$ of generalized signal translations, which include both horizontal and vertical translations, and shall prove that signal dilations (resp. erosions) invariant under such translations are equivalent to generalized supremal (resp. infimal) convolutions. Related adjunctions will also be found from pairs of such operators.

These results generalize previous work by Heijmans and Ronse (1990) where $\mathbb{T}$ was constrained to be a commutative group of automorphisms. We also treat differently the horizontal form the vertical translations. Heijmans and Ronse (1990) had also made a basic assumption that $\mathcal{S}$ contained a sup-generating subset $\ell$, which was left invariant by $\mathbb{T}$ and that $\mathbb{T}$ was transitive on $\ell$. In this chapter, where we deal with signal and vector spaces, this sup-generating class already exists and is formed by vertical translations of the upper basis of the complete weighted lattice; as explained later, this basis consists of horizontally translated impulses.

\subsubsection{Generalized Translations}

The signal domain $E$ possesses various commutative group structures which allow us to define corresponding horizontal motions that form two major types of Euclidean motions. The most obvious and practical choice is to set $E=\mathbb{R}^{m}$ or $\mathbb{Z}^{m}$ and consider the horizontal translations on

\footnotetext{
${ }^{18}$ In this section we will not use the bolface notation for vector points of the signal domain $E$, because this will not always be equal to $\mathbb{E}^{m}$ and the generalized translation group on it will not always be the standard horizontal Euclidean translation group.
} 
the image plane where each such operator $\mu_{h}(F)(x)=F(x-h)$ performs a planar shift of an input signal $F(x)$ along the vector $h$. The class $\mathbb{T}_{h t}=\left\{\mu_{h}: h \in \mathbb{E}^{m}\right\}$ of all horizontal translations under composition is a commutative group of automorphisms both on the Boolean lattice $\mathcal{P}\left(\mathbb{E}^{m}\right)$ (the collection of all subsets of $\left.\mathbb{E}^{m}\right)$ as well as on the signal lattice $\mathcal{S}$; i.e. $\mu_{x} \mu_{y}=\mu_{x+y}$. A second choice is to set $E=\mathbb{R}^{2} \backslash\{(0,0)\}$ and to consider polar motions $\mu_{r, \theta}$ : that transform the support of an input signal via a rotation by $\theta$ and a radial scaling by $r$. The class $\mathbb{T}_{h r}=\left\{\mu_{r, \theta}: r>0, \theta \in[0,2 \pi)\right\}$ of all polar motions is also a commutative group of automorphisms; i.e. $\mu_{r, \theta} \mu_{\rho, \phi}=\mu_{r \rho, \theta+\phi}$. Both of these choices were studied in Heijmans $(1987,1994)$; the polar case in Roerdink and Heijmans (1988). As a third possibility we can consider the group of all Euclidean planar motions, i.e. combined translations and rotations; this is a non-commutative group of automorphisms studied in Roerdink (1993). Another example of a non-commutative group of automorphisms is the group of affine of mappings on the plane, studied in Maragos (1990). Henceforth, we focus only on the commutative cases, i.e. the first two choices. However, for notational simplicity, we shall use only the horizontal Euclidean translation group and write it simply as $\mathbb{T}_{h}$, even if our results will also include the case of polar motions. Further, for both of these horizontal translation groups we will use the additive notation for its group operation. Note a difference though: the horizontal Euclidean translations apply to more general signal domains, both continuous $\left(\mathbb{R}^{m}\right)$ and discrete $\left(\mathbb{Z}^{m}\right)$, whereas for the polar motions we need as domain the punctured plane $\mathbb{R}^{2} \backslash\{(0,0)\}$.

In the previous section we worked with vertical translations $\lambda_{v}[F(x)]=F(x) \star v$ whose collection $\mathbb{L}$ forms a commutative monoid of signal dilations, as well as with dual vertical translations $\lambda_{v}^{\prime}[F(x)]=F(x) \star^{\prime} v$ which are erosions. The composition of these two (horizontal and vertical) types of translations yields a generalized translation $\tau$ and its dual $\tau^{\prime}$ :

$$
\tau_{h, v}(F)(x) \triangleq F(x-h) \star v, \quad \tau_{h, v}^{\prime}(F)(x) \triangleq F(x-h) \star^{\prime} v
$$

Note that the horizontal and vertical translations commute:

$$
\tau_{h, v}=\mu_{h} \lambda_{v}=\lambda_{v} \mu_{h}, \quad \tau_{h, v}^{\prime}=\mu_{h} \lambda_{v}^{\prime}=\lambda_{v}^{\prime} \mu_{h}
$$

The collection of all such generalized translations

$$
\mathbb{T}=\mathbb{T}_{h} \times \mathbb{L}=\left\{\tau_{h, v}: h \in E, v \in \mathcal{C}\right\}
$$

forms a monoid under composition:

$$
\tau_{x, a} \tau_{y, b}=\tau_{x+y, a \star b}
$$

If $\mathcal{C}$ is a blog, then $\mathbb{T}$ restricted to non-extreme vertical translations becomes a group of automorphisms. However, in the general case, $\mathbb{T}$ is just a commutative monoid of elementary signal dilations on $\mathcal{S}$. We call an operator $\psi \mathbb{T}$-translation invariant, in short $\mathbb{T}$-invariant, if it commutes with all translations $\tau \in \mathbb{T}$.

$$
\psi \text { is } \mathbb{T} \text {-invariant: } \quad \psi \tau=\tau \psi, \forall \tau \in \mathbb{T}
$$

Dually, the collection $\mathbb{T}^{\prime}=\left\{\tau_{h, v}^{\prime}: h \in E, v \in \mathcal{C}\right\}$ forms a monoid of signal erosions. We call an operator $\mathbb{T}^{\prime}$-invariant if it commutes with all translations $\tau^{\prime} \in \mathbb{T}^{\prime}$.

Consider now two elementary signals, called the impulse $q$ and the dual impulse $q^{\prime}$

$$
q(x) \triangleq\left\{\begin{array}{ll}
\mathcal{C}_{\mathrm{id}}, & x=\mathbf{0} \\
\mathcal{C}_{\mathrm{O}}, & x \neq \mathbf{0}
\end{array}, \quad q^{\prime}(x) \triangleq \begin{cases}\mathcal{C}_{\mathrm{id}}^{\prime}, & x=\mathbf{0} \\
\mathcal{C}_{\mathrm{I}}, & x \neq \mathbf{0}\end{cases}\right.
$$

which are the pulse semi-atoms of $\mathcal{S}$ placed at the origin $\mathbf{0}$ of $\mathbb{E}^{m}$ and with identity height. Then every signal can be represented as a supremum of translated impulses or as infimum of dualtranslated impulses:

$$
F(x)=\bigvee_{y \in E} F(y) \star q(x-y)=\bigwedge_{y \in E} F(y) \star^{\prime} q^{\prime}(x-y)
$$


Now, in this CWL signal space $\mathcal{S}$ on which we also have generalized translations, the set $\mathcal{B}$ of all translated impulses

$$
\mathcal{B}=\{q(x-y): y \in E\}
$$

is an infinite upper basis for $\mathcal{S}$, which is infinite-dimensional. Dually, the set $\mathcal{B}^{\prime}=\left\{q^{\prime}(x-y): y \in E\right\}$ is a lower basis of the same cardinality as $\mathcal{B}$.

\subsubsection{Generalized Convolution Representation of $\mathbb{T}$-Invariant Dilations and Erosions}

Consider now a $\mathbb{T}$-invariant dilation on $\mathcal{S}$, i.e. an operator $\Delta$ that obeys the nonlinear superposition (122) and is horizontally translation-invariant, or equivalently distributes over suprema and obeys the $\mathbb{T}$-invariance. We call $\Delta$ a dilation translation-invariant (DTI) system. Let

$$
H \triangleq \Delta(q)
$$

be the system's impulse response. We shall show next that, the DTI system's output $\Delta(F)$ due

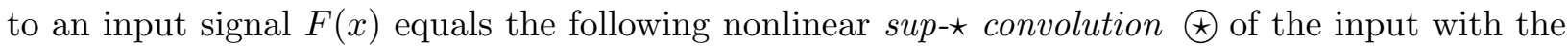
impulse response:

$$
(F \circledast H)(x) \triangleq \bigvee_{y \in E} F(y) \star H(x-y)
$$

Dually, consider a $\mathbb{T}^{\prime}$-invariant signal erosion, i.e. an operator $\mathcal{E}$ that distributes over infima and obeys the $\mathbb{T}^{\prime}$-invariance; we call $\mathcal{E}$ an erosion translation-invariant (ETI) system. Let $H^{\prime}=$ $\mathcal{E}\left(q^{\prime}\right)$ be the system's dual impulse response. As shown next, the ETI system's output $\mathcal{E}(F)$ equals the following nonlinear inf- $\star^{\prime}$ convolution $\star^{\prime}$ of the input $F$ with the dual impulse response $H^{\prime}$ :

$$
\left(F \circledast H^{\prime}\right)(x) \triangleq \bigwedge_{y \in E} F(y) \star^{\prime} H^{\prime}(x-y)
$$

Thus, DTI and ETI systems are represented by the above nonlinear convolutions, and conversely. Overall, we have the following fundamental result.

Theorem 11 (Maragos, 2005a).

Consider a $C W L$ signal space $\mathcal{S}=\operatorname{Fun}(E, \mathcal{C})$ over the clodum $\left(\mathcal{C}, \vee, \wedge, \star, \star^{\prime}\right)$ of scalars. Over this space, consider the monoid $\mathbb{T}$ of generalized translations defined in (130) and the monoid $\mathbb{T}^{\prime}$ of dual translations. Then:

(a) (DTI Systems): A signal operator $\Delta$ on $\mathcal{S}$ is a $\mathbb{T}$-translation invariant dilation iff it can be

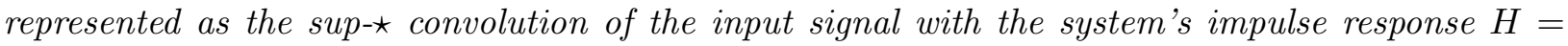
$\Delta(q)$.

(b) (ETI Systems): A signal operator $\mathcal{E}$ on $\mathcal{S}$ is a $\mathbb{T}^{\prime}$-translation invariant erosion iff it can be represented as the inf- $\star^{\prime}$ convolution of the input signal with the system's dual impulse response $H^{\prime}=\mathcal{E}\left(q^{\prime}\right)$.

The above theorem has a direct conceptual analogy with Riesz's representation theorem for linear operators, which states that linear and horizontally translation-invariant (in short, LTI) operators are uniquely represented as linear (sum-product) convolutions of the input $F$ with their impulse response $H$ :

$$
\Gamma \text { is LTI } \Longleftrightarrow \Gamma(F)(x)=(F * H)(x)=\sum_{y} F(y) H(x-y)
$$




\subsubsection{Generalized Convolution Adjunctions}

Let us now find the adjoint operators of the above nonlinear convolutions. A $\mathbb{T}$-invariant dilation $\Delta_{H}(F)=F \circledast H$ can be represented via scalar dilations as

$$
\begin{aligned}
\Delta_{H}(F)(x) & =\bigvee_{y \in E} F(y) \star H(x-y) \\
& =\bigvee_{y \in E} \lambda_{H(x-y)}(F(y))
\end{aligned}
$$

where the V-translation $\lambda_{a}(v)=a \star v$ is a scalar dilation. Let $\lambda_{a}^{\leftarrow}$ be the scalar adjoint erosion of $\lambda_{a}$. By setting

$$
\lambda_{H(x-y)}(v)=d_{y, x}(v), \quad \lambda_{H(x-y)}^{\leftarrow}(w)=e_{x, y}(w)
$$

we can identify the scalar adjunction $\left(\lambda_{H(x-y)}^{\leftarrow}, \lambda_{H(x-y)}\right)$ of V-translations with the scalar adjunction $\left(e_{x, y}, d_{y, x}\right)$ of Proposition 15. Then, it follows that the adjoint signal erosion of $\Delta_{H}$ is

$$
\mathcal{E}_{H}(G)(y)=\bigwedge_{x \in E} \lambda_{H(x-y)}^{\overleftarrow{ }}(G(x))
$$

If $\mathcal{C}$ is a blog, i.e. $\mathcal{C}_{\mathrm{G}}=\mathcal{C} \backslash\left\{\mathcal{C}_{\mathrm{O}}, \mathcal{C}_{\mathrm{I}}\right\}$ is a group under $\star$-'multiplication', let $v^{*}$ denote the conjugate of each scalar $v \in \mathcal{C}$ as defined in (104); this coincides with the group inverse if $v$ is a group element. Then, the scalar adjoint erosion can be expressed as $\lambda_{a}^{\leftarrow}(w)=a^{*} \star w$, and hence the adjoint signal erosion becomes

$$
\mathcal{E}_{H}(G)(y)=\bigwedge_{x \in E} G(x) \star[H(x-y)]^{*}
$$

By interchanging $x$ with $y$ we can write this as

$$
\mathcal{E}_{H}(G)(x)=\bigwedge_{y \in E} G(y) \star[H(y-x)]^{*}
$$

which, when compared with (138), reveals that the adjoint of a signal sup- $\star$ convolution has the computational structure of an inf- $\star$ correlation.

Similarly, if we consider a $\mathbb{T}^{\prime}$-erosion $\mathcal{E}_{H^{\prime}}(G)=G \circledast{ }^{\prime} H^{\prime}$ and decompose it into scalar erosions as

$$
\begin{aligned}
\mathcal{E}_{H^{\prime}}(G)(x) & =\bigwedge_{y \in E} G(y) \star^{\prime} H^{\prime}(x-y) \\
& =\bigwedge_{y \in E} \lambda_{H^{\prime}(x-y)}^{\prime}(G(y))
\end{aligned}
$$

where $\lambda_{a}^{\prime}(w)=a \star^{\prime} w$ are dual V-translations, then the adjoint signal dilation of $\mathcal{E}_{H^{\prime}}$ is

$$
\Delta_{H^{\prime}}(F)(y)=\bigvee_{x \in E} \lambda_{H^{\prime}(x-y)}^{\prime \leftarrow}(F(x))
$$

where $\lambda_{a}^{\prime \leftarrow}$ is the scalar adjoint dilation of $\lambda_{a}^{\prime}$. Again, if $\mathcal{C}$ is a blog, each scalar $v$ possesses a conjugate $v^{*}$, and the scalar adjoint dilation can be shown to equal $\lambda_{a}^{\prime \leftarrow}(v)=a^{*} \star^{\prime} v$. Then, the adjoint signal dilation becomes

$$
\Delta_{H^{\prime}}(F)(y)=\bigvee_{x \in E} F(x) \star^{\prime}\left[H^{\prime}(x-y)\right]^{*}
$$

We see in both cases that while a $\mathbb{T}$-invariant dilation (or $\mathbb{T}^{\prime}$-invariant erosion) has the computational structure of a signal convolution, its corresponding adjoint has the structure of a nonlinear signal correlation. Now, are these adjoint operators invariant w.r.t. the corresponding generalized translation? The next result describes the limits of such invariances. 
Proposition 16 (Maragos, 2005a).

Let $(\varepsilon, \delta)$ be an adjunction on the $C W L$ space $\mathcal{S}$. Then:

(a) $\delta$ is invariant to any horizontal translation $\mu$ iff $\varepsilon$ is invariant to such translation; i.e. $\delta \mu=$ $\mu \delta \Longleftrightarrow \varepsilon \mu=\mu \varepsilon$.

(b) Consider a scalar adjunction $\left(\lambda^{\leftarrow}, \lambda\right)$ on $\mathcal{C}$ where $\lambda$ is a vertical translation and $\lambda^{\leftarrow}$ is its adjoint. Then $\delta$ is invariant to a vertical translation $\lambda$ iff $\varepsilon$ is invariant to the adjoint translation $\lambda^{\leftarrow}$; i.e. $\delta \lambda=\lambda \delta \Longleftrightarrow \varepsilon \lambda^{\leftarrow}=\lambda^{\leftarrow} \varepsilon$.

(c) Consider a scalar adjunction $\left(\lambda^{\prime}, \lambda^{\prime \leftarrow}\right)$ on $\mathcal{C}$ where $\lambda^{\prime}$ is a dual vertical translation and $\lambda^{\prime \leftarrow}$ is its adjoint. Then $\varepsilon$ is invariant to a dual vertical translation $\lambda^{\prime}$ iff $\delta$ is invariant to the adjoint translation $\lambda^{\prime \leftarrow}$.

(d) If $\mathcal{C}$ is a blog, $\delta$ is $\mathbb{T}$-invariant iff $\varepsilon$ is $\mathbb{T}$-invariant.

Concluding, our emphasis on working always with adjunctions $(\varepsilon, \delta)$ is justified by the following reasons: (i) If we have an adjunction, we can immediately create an opening $\alpha(F)=\delta \varepsilon(F)$ and a closing $\beta(F)=\varepsilon \delta(F)$, by simply concatenating the erosion and dilation. (ii) If a signal dilation (resp. erosion) is not invertible, then its adjoint erosion (resp. dilation) is the closest to an 'inverse operator'. (iii) Adjunctions provide us with many tools to analyze their constituent operators.

\subsection{Special Cases}

By specifying the clodum $\mathcal{C}$ and its scalar 'multiplication' operations $\star$ and its dual $\star$ ', we obtain a large variety of classes of nonlinear image processing systems that are described by the previous unified representations. Next we briefly describe three such choices.

\subsubsection{Max-Plus Image Operators}

We set $\mathcal{C}=\overline{\mathbb{R}}=\mathbb{R} \cup\{-\infty,+\infty\}$. The 'multiplications' $\star$ and $\star^{\prime}$ are the regular extended addition + and its dual $+^{\prime}$ over $\overline{\mathbb{R}}$, respectively; i.e., + and $+^{\prime}$ are identical for finite reals, but

$$
a+(-\infty)=-\infty \quad \text { and } \quad a+^{\prime}(+\infty)=+\infty \quad \forall a \in \overline{\mathbb{R}}
$$

Thus, the clodum of scalars is $\left([-\infty, \infty], \vee, \wedge,+,+^{\prime}\right)$. In this case $\mathcal{C}$ is a blog and contains an additive group $(\mathbb{R},+)$ where each scalar $a$ has an inverse $-a$ that coincides with its conjugate $a^{*}$. The adjunction $\left(\mathcal{E}_{H}, \Delta_{H}\right)$ of a sup-sum convolution (dilation) $\Delta_{H}$ and its adjoint erosion $\mathcal{E}_{H}$ become

$$
\begin{aligned}
\Delta_{H}(F)(x) & =(F \oplus H)(x) \triangleq \bigvee_{y \in E} F(y)+H(x-y) \\
\mathcal{E}_{H}(F)(x) & =(F \ominus H)(x) \triangleq \bigwedge_{y \in E} F(y)-H(y-x)
\end{aligned}
$$

These are the traditional weighted Minkowski dilation and erosion of an image $F$ by an additive structuring function $H$ (Sternberg, 1980), which have found numerous applications in nonlinear filtering, image processing and computer vision Serra (1982); Sternberg (1986); Ritter and Wilson (1987); Maragos and Schafer (1990); Haralick and Shapiro (1992); Heijmans (1994); Dougherty and Astola (1994); Maragos $(1998,2005 b)$. Similarly, the adjunction $\left(\mathcal{E}_{H^{\prime}}, \Delta_{H^{\prime}}\right)$ of an inf-sum convolution (erosion) $\mathcal{E}_{H^{\prime}}$ and its adjoint dilation $\Delta_{H^{\prime}}$ become

$$
\begin{aligned}
\mathcal{E}_{H^{\prime}}(F)(x)=\left(F \oplus^{\prime} H\right)(x) & \triangleq \bigwedge_{y \in E} F(y)+{ }^{\prime} H^{\prime}(x-y) \\
\Delta_{H^{\prime}}(F)(x) & =\bigvee_{y \in E} F(y)+{ }^{\prime}\left[-H^{\prime}(y-x)\right]
\end{aligned}
$$

Note that sup-sum $\oplus$ and inf-sum $\oplus^{\prime}$ convolutions have been known in optimization (Bellman and Karush, 1963) and convex analysis (Rockafellar, 1970; Lucet, 2010) under the names 'supremal convolution' and 'infimal convolution'. 19

\footnotetext{
${ }^{19}$ In convex analysis (Rockafellar, 1970; Lucet, 2010) the infimal convolution of two functions $f$ and $g$ is usually denoted by $(f \square g)(x)=\bigwedge_{y} f(y)+g(x-y)$. In our exposition we use the symbol $\oplus^{\prime}$.
} 
The signal translations become

$$
\tau_{h, v}(F)(x)=F(x-h)+v
$$

and their collection for $h \in \mathbb{E}^{m}$ and $v \in \mathbb{R}$ is a commutative group of automorphisms, studied in Heijmans and Ronse (1990); Heijmans (1994); note that to get a group we restrict the vertical translation amounts $v$ to be finite. Henceforth, unless we clarify it in the context, when we talk about 'translation-invariance (TI)' of some operator without specifying the translation-group, we shall refer to this standard case: the horizontal Euclidean translations group and the vertical scalar addition group. Another standard term is 'shift-invariance' of some operator $\psi$ which shall always mean invariance with respect to the horizontal Euclidean translations group, i.e. $\psi \tau_{h, 0}=\tau_{h, 0} \psi$.

As explained in Section 5, TI operators play a major role in the morphological representation theory. For example, increasing TI operators can be represented as a supremum of Minkowski erosions or as infimum of dilations by functions in a suitable collection called 'kernel' or a minimal subcollection (Maragos, 1989a).

In addition to morphological image processing, there are many other scientific fields where the max-plus algebraic system is often used. Examples include machine scheduling and operations research (Cuninghame-Green, 1979), convex analysis and optimization (Rockafellar, 1970), shortest path problems on graphs (Peteanu, 1967), morphological neural nets (Davidson and Hummer, 1993; Ritter et al., 1998; Pessoa and Maragos, 2000; Ritter and Urcid, 2003).

In short, the max-plus case is the algebraically richest and most well explored case in mathematical morphology and image algebra as well as in minimax algebra, both in theory and in applications.

\subsubsection{Max-Product Image Operators}

Another less explored paradigm, but equally algebraically rich with the max-plus case, results when we choose as set of scalars the extended nonnegative numbers $\mathcal{C}=[0,+\infty]$ and as self-dual *-'multiplication' the standard product ' $x$ ' of nonnegative numbers extended to include the $+\infty$. Thus, the clodum of scalars is $\left([0, \infty], \vee, \wedge, \times, \times^{\prime}\right)$. The signal translations become

$$
\tau_{h, v}(F)(x)=F(x-h) \times v
$$

and their collection for $h \in E$ and $v \in(0,+\infty)$ is a commutative group of automorphisms, studied in Heijmans and Ronse (1990); Heijmans (1994); note that to get a translation group we restrict the multiplication scalars $v$ to be non-extreme. As in the max-plus case, the scalar set $\mathcal{C}$ is again a blog and contains a multiplicative group $((0, \infty), \times)$ with inverses $a^{-1}$ that coincide with the conjugate $a^{*}$ of each scalar $a$. Now, the adjunction $\left(\mathcal{E}_{H}, \Delta_{H}\right)$ of a sup-product convolution (dilation) $\Delta_{H}$ and its adjoint erosion $\mathcal{E}_{H}$ become

$$
\begin{aligned}
\Delta_{H}(F)(x)=(F \otimes H)(x) & \triangleq \bigvee_{y \in E} F(y) \times H(x-y) \\
\mathcal{E}_{H}(F)(x) & =\bigwedge_{y \in E} F(y) / H(y-x)
\end{aligned}
$$

These are translation-invariant Minkowski-like dilation and erosion of an image $F$ by a multiplicative structuring function $H$. Some of its properties, their translation-invariances and kernel representations of such systems have been studied in Heijmans (1994).

Note that there is an isomorphism between the max-plus and the max-product enabled by a logarithmic-exponential pointwise bijection of the image signals. Despite this isomorphism, we believe that there is a significant applications potential in this algebraic system, which has not been explored so far. This potential can be appreciated by the following observations: (1) Image signals are naturally nonnegative and the max-product dilations-erosions maintain this nonnegativity of the input signals. (2) In certain vision applications there is sometimes the need to include in the visual processing the logarithm of intensity images; e.g., such a nonlinearity approximates some of the early stages in biological vision systems. This creates the density (log-intensity) representation of images. Then, max-plus dilations and erosions of the density image are equivalent to max-product dilations and erosions of the intensity image. 


\subsubsection{Weighted Lattice Operators using Fuzzy Norms}

This section presents an important special case of our unified lattice image processing systems which covers a broad area in the intersection of mathematical morphology and fuzzy logic. We set $\mathcal{C}=[0,1]$ and the clodum of scalars is $\left([0,1], \vee, \wedge, T, T^{\prime}\right)$ where $T$ (resp. $\left.T^{\prime}\right)$ is a fuzzy intersection (resp. union). The signal space $\mathcal{S}=\operatorname{Fun}(E,[0,1])$ consists of all image signals defined on $E=\mathbb{R}^{m}$ or $\mathbb{Z}^{m}$ and assuming real values in $[0,1]$; alternatively, these signals can be viewed as membership functions of fuzzy sets. This is a more difficult clodum case than the previous two (max-plus and max-product) because it is not a blog. Thus, there are no inverses under the $\star$-'multiplication'.

Mathematical Morphology and Fuzzy Logic: Mathematical morphology and fuzzy sets share many common theoretical concepts. As an earlier example, the use of $\mathrm{min} / \mathrm{max}$ to extend the intersection/union of ordinary (crisp) sets to fuzzy sets Zadeh (1965) has also been used to extend the set-theoretic morphological shrink/expand operations on binary images to $\mathrm{min} / \mathrm{max}$ filtering on graylevel images (Nakagawa and Rosenfeld, 1978; Goetcherian, 1980). While the field of morphological image analysis was maturing, several researchers developed various other approaches using fuzzy logic ideas for extending or generalizing the morphological image operations (Sinha and Dougherty, 1992; Bloch and Maitre, 1995). The main ingredients of these approaches have been to (1) map the max-plus structure of Minkowski signal dilation to a sup- $T$ signal convolution, where $T$ is some fuzzy intersection norm, and (2) use duality to map the inf-minus structure of Minkowski signal erosion to a inf- $T^{\prime}$ convolution, where $T^{\prime}$ is a dual fuzzy union norm. We refer the reader to Nachtegael and Kerre (2001) for connections and comparisons of all these approaches to fuzzy morphologies. The main disadvantage of these approaches is that composition of the operators from steps (1) and (2) is not guaranteed to be an algebraic opening or closing. In addition to the above approaches, there have been efforts to combine mathematical morphology and fuzzy logic or lattices and neuro-fuzzy systems by fuzzifying respectively the inclusion indicator or the partial ordering of the lattice, as done respectively in Chatzis and Pitas (2000) and Kaburlasos and Petridis (2000). In the field of pattern recognition, of relevance is also the work in Yang and Maragos (1995) on min-max classifiers that used max-min operations on vectors.

In Maragos et al. (2000, 2001, 2003) lattice theory was used to develop generalizations of morphological signal and vector operations based on fuzzy norms that have an adjunction structure A similar work appeared independently in Deng and Heijmans (2002). In this section we present these preliminary results as special cases of the general algebraic structure. From fuzzy set theory (Klir and Yuan, 1995) we use t-norms and t-conorms to extend intersection and union of crisp sets to signal convolutions. To form openings and closings we use pairs of t-norms and fuzzy implications.

Fuzzy Intersection and Union Norms: A fuzzy intersection norm, in short a $T$-norm, is a binary operation $T:[0,1]^{2} \rightarrow[0,1]$ that satisfies the following conditions (Klir and Yuan, 1995): For all $a, b, c \in[0,1]$

F1. $T(a, 1)=a$ and $T(a, 0)=0$ (boundary conditions).

F2. $T(a, T(b, c))=T(T(a, b), c)$ (associativity).

F3. $T(a, b)=T(b, a)$ (commutativity).

F4. $b \leq c \Longrightarrow T(a, b) \leq T(a, c)$ (increasing).

For the $T$-norm to be a scalar dilation (with respect to any argument) on $\mathbb{V}$, it must also satisfy (Maragos et al., 2000):

F5. $T$ is a continuous function.

A fuzzy union norm (Klir and Yuan, 1995) is a binary operation $U:[0,1]^{2} \rightarrow[0,1]$ that satisfies F2-F5 and a dual boundary condition:

$\mathrm{F} 1^{\prime} . U(a, 0)=a$ and $U(a, 1)=1$.

Clearly, $U$ is an erosion on $\mathbb{V}$. 
Lattice Fuzzy Convolutions and Adjunctions: We have built the general DTI or ETI systems (equivalent to sup $-\star$ or inf- $\star^{\prime}$ convolutions) as supremum or infimum of signal translations of the type $\tau_{h, v}(f)(x)=f(x-y) \star v$. In this section we shall use new translations where the binary operation $a \star b$ is replaced by fuzzy intersection norms $T$ and the dual operation $a \star^{\prime} b$ is replaced by fuzzy union norms $U$. Namely, the new generalized signal translations on $\mathcal{S}=\operatorname{Fun}(E,[0,1])$ are the operators $\tau$ and the dual translations are the operators $\tau^{\prime}$ :

$$
\begin{aligned}
& \tau_{h, v}(f)(x)=T(f(x-y), v) \\
& \tau_{h, v}^{\prime}(f)(x)=U(f(x-y), v)
\end{aligned}
$$

where $(h, v) \in E \times[0,1]$ and $f(x)$ is an arbitrary input signal. (As in the previous two special cases, the signal domain will be $E=\mathbb{E}^{m}$.) These translations include both horizontal shifts as well as vertical shifts induced by the fuzzy norms. A signal operator on $\mathcal{S}$ is called translation invariant (resp. dual-translation invariant) iff it commutes with any such translation $\tau$ (resp. $\tau^{\prime}$ ) based on a fuzzy norm. Consider now the two elementary signals, the impulse $q$ and the dual impulse $q^{\prime}$ :

$$
q(x) \triangleq\left\{\begin{array}{ll}
1, & x=\mathbf{0} \\
0, & x \neq \mathbf{0}
\end{array}, \quad q^{\prime}(x) \triangleq \begin{cases}0, & x=\mathbf{0} \\
1, & x \neq \mathbf{0}\end{cases}\right.
$$

Then every signal $f$ can be represented as a supremum of translated impulses or as infimum of dual-translated dual impulses:

$$
f(x)=\bigvee_{y} T[q(x-y), f(y)]=\bigwedge_{y} U\left[q^{\prime}(x-y), f(y)\right]
$$

Translation invariant signal dilations and erosions can result, respectively, from the sup- $T$ convolution $\bigcirc_{T}$ and the inf- $U$ convolution $\bigcirc_{U}^{\prime}$ of two signals $f$ and $g$ defined by

$$
\begin{aligned}
& \left(f \bigcirc_{T} g\right)(x) \triangleq \bigvee_{y} T[g(x-y), f(y)], \\
& \left(f \bigcirc_{U}^{\prime} g\right)(x) \triangleq \bigwedge_{y} U[g(x-y), f(y)]
\end{aligned}
$$

The following results are a direct corollary of the generalized convolution representation Theorem 11.

Theorem 12 (Maragos, 2005a).

(a) Given a fuzzy intersection norm $T$, an operator $\Delta$ on $\operatorname{Fun}(E,[0,1])$ is a dilation invariant to the general translations (149) iff it can be represented as the sup-T convolution of the input signal with the operator's impulse response $H=\Delta(q)$.

(b) Given a fuzzy union norm $U$, an operator $\mathcal{E}$ on $\operatorname{Fun}(E,[0,1])$ is an erosion invariant to the dual translations (150) iff it can be represented as the inf- $U$ convolution of the input signal with the system's dual impulse response $H^{\prime}=\mathcal{E}\left(q^{\prime}\right)$.

However, the erosion $\mathcal{E}$ of the above theorem may not be the adjoint of the dilation $\Delta$. To form an adjunction, we first define a signal fuzzy dilation as a sup- $T$ convolution:

$$
\Delta_{H, T}(F)(x) \triangleq \bigvee_{y \in E} T[H(x-y), F(y)]=\left(F \bigcirc_{T} H\right)(x)
$$

By recognizing $T[H(x-y), F(y)]$ as the scalar dilations $d_{y, x}(F(y))$ in the general decomposition (119) of a signal dilation, it follows that the adjoint signal fuzzy erosion is

$$
\mathcal{E}_{H, \Omega}(G)(y) \triangleq \bigwedge_{x \in E} \Omega[H(x-y), G(x)]
$$


where $\Omega[H(x-y), G(x)]$ represents the adjoint scalar erosions $e_{x, y}(G(x))$ in (119) and is actually the adjoint of the fuzzy $T$-norm:

$$
T(a, v) \leq w \Longleftrightarrow v \leq \Omega(a, w)
$$

An alternative interpretation of $T[H(x-y), F(y)]$ and $\Omega[H(x-y), G(x)]$ is that they are equal, respectively, to a scalar $\mathrm{V}$-translation $\lambda$ and its adjoint $\lambda^{\leftarrow}$ of the signal values:

$$
\begin{aligned}
T[H(x-y), F(y)] & =\lambda_{H(x-y)}(F(y)) \\
\Omega[H(x-y), G(x)] & =\lambda_{H(x-y)}^{\overleftarrow{(}(G(x))}
\end{aligned}
$$

Now, given $T$ we can find its adjoint function $\Omega$ by

$$
\Omega(a, w)=\sup \{v \in[0,1]: T(a, v) \leq w\}
$$

In fuzzy logic, the norm $T$ can be interpreted as a logical conjunction, whereas its corresponding adjoint $\Omega$ can be interpreted as a logical implication (Klir and Yuan, 1995).

Three examples of $T$-norms are:

$$
\begin{aligned}
& \text { Min : } T_{1}(a, v)=\min (a, v) \\
& \text { Product : } T_{2}(a, v)=a \cdot v \\
& \text { Yager : } T_{3}(a, v)=1-\left(1 \wedge\left[(1-v)^{p}+(1-a)^{p}\right]^{1 / p}\right), p>0 .
\end{aligned}
$$

The corresponding three adjoint functions are:

$$
\begin{aligned}
& \Omega_{1}(a, w)=\left\{\begin{array}{l}
w, \quad w<a \\
1, \quad w \geq a
\end{array}\right. \\
& \Omega_{2}(a, w)= \begin{cases}\min (w / a, 1), & a>0 \\
1, & a=0\end{cases} \\
& \Omega_{3}(a, w)= \begin{cases}1-\left[(1-w)^{p}-(1-a)^{p}\right]^{1 / p}, & w<a \\
1, & w \geq a\end{cases}
\end{aligned}
$$

Let us consider now the construction of lattice-fuzzy openings and closings based on an adjunction $(\varepsilon, \delta)$ of a lattice-fuzzy dilation $\delta$ and erosion $\varepsilon$. The adjunctional lattice-fuzzy opening $\alpha$ and lattice-fuzzy closing $\beta$ are defined as

$$
\alpha(f) \triangleq \delta(\varepsilon(f)), \quad \beta(f) \triangleq \varepsilon(\delta(f))
$$

This is the correct approach to create openings and closings from fuzzy dilations and erosions. To compare it with previous works, consider an involutive fuzzy complement $a \mapsto a^{*}$, e.g. $a^{*}=1-a$. This is a negation (i.e. conjugation) on the scalar clodum $[0,1]$ and induces a negation on the signal clodum $\mathcal{S}$ too. If we define via complementation an alternative erosion operator (as an inf- $U$ convolution) by

$$
\varepsilon^{\prime}(f)(y)=\bigwedge_{x} U[f(x), h(y-x)]
$$

where $U(a, b)=1-T(1-a, 1-b)$ is a fuzzy union that is the dual (i.e. complement) of the fuzzy intersection $T$, then $\varepsilon^{\prime}(f)=1-\delta(1-f)=\delta^{*}(f)$, where $\psi^{*}$ denotes the negative operator of $\psi$; i.e., this second erosion $\varepsilon^{\prime}$ is the dual (i.e. negative) of the first dilation $\delta$. Further, the adjoint dilation $\delta^{\prime}$ of $\varepsilon^{\prime}$ is an operator that is dual (i.e. negative) of the first erosion $\varepsilon$. Many previous works used pairs $\left(\varepsilon^{\prime}, \delta\right)$ which are duality pairs (via negation) but not adjunctions and hence cannot form openings/closings via compositions. 
Examples: To gain some insight on the lattice-fuzzy image operators, we briefly present a few experimental results illustrating the differences between the classical morphological operators and the lattice operators based on fuzzy $T$-norms.

Figure 6 shows the outputs of dilation, erosion, opening and closing operators on 1D images, first for the morphological type using a 51-pixel flat structuring element and second for the fuzzy type (153), (154) and (158). For the fuzzy operations in Fig. 6, three T-norms were used: the minimum norm, the product norm, and the Yager norm (with parameter $p=2$ ). The structuring function $H: \mathbb{Z} \rightarrow[0,1]$ was the parabola

$$
H(x)= \begin{cases}1-k(x / s)^{2}, & |x| \leq s \\ 0, & |x|>s\end{cases}
$$

whose parameter $s$ determines the scale, while $k$ affects the shape of $H$. (We used $s=25$ and $k=0.5$.) In general, by experimenting with a large variety of $T$-norms and structuring functions $H$ we have observed that, the fuzzy operators are more adaptive and track closer the peaks/valleys of the signal than the corresponding flat morphological operators of the same scale.

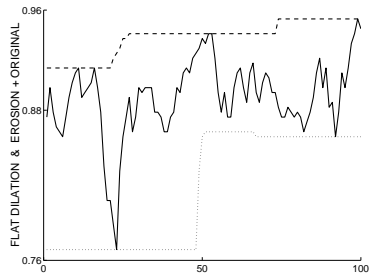

(a)

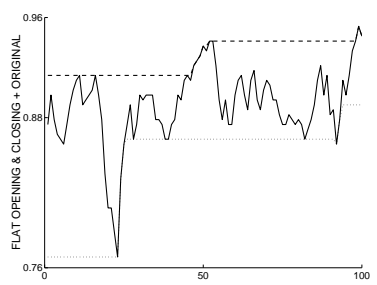

(e)

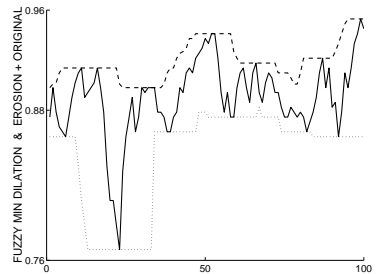

(b)

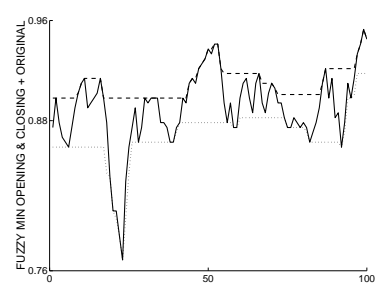

(f)

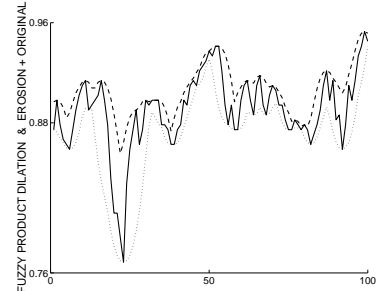

(c)

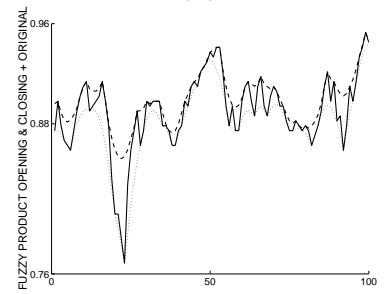

$(\mathrm{g})$

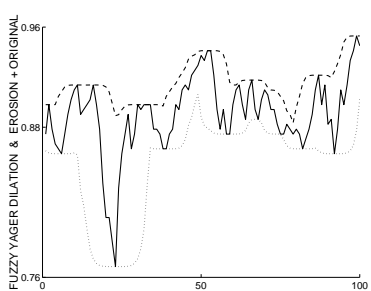

(d)

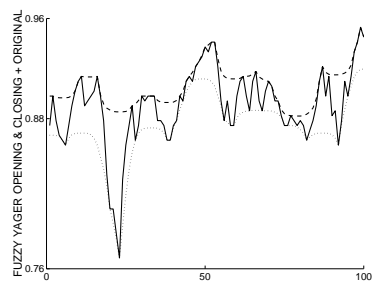

(h)

Figure 6: Comparison of 1D basic morphological and lattice-fuzzy signal operators. Rows 1 and 2, left to right: flat, minimum, product, Yager. Row 1: original signal (solid line), dilation (dashed line), erosion (dotted line). Row 2: closing (dashed line), opening (dotted line). From Maragos et al. (2001).

Figure 7 reports experiments with 2D images. For all the fuzzy operations in this figure, we used the Yager $T$-norm with parameter $p=10$ and a conical structuring function $H$. The second row compares the morphological flat dilation and erosion of an original image in Fig. 7(a) with its fuzzy dilation and erosion. In both cases the structuring element had a $7 \times 7$-pixel support, being flat in the morphological case and conical in the fuzzy case. The third row of Fig. 7 deals with edge enhancement: Figure 7(f) shows the standard discrete morphological gradient $F \oplus B-F \ominus B$, as the difference between the morphological flat dilation and erosion, respectively, of $F$ by a $3 \times 3$-pixel square $B$. Figure $7(\mathrm{~g})$ shows the same type of gradient but uses a fuzzy dilation $\delta$ and erosion $\varepsilon$ with a $3 \times 3$-pixel structuring function $H$. Figures $7(\mathrm{~h})$ and (i) combine the fuzzy dilation and erosion differently to derive respectively the following two types of new edge gradients:

$$
\begin{aligned}
& \text { FuzzyEdge }_{\text {min }}(F)=\min [\delta(F), 1-\varepsilon(F)] \\
& \text { FuzzyEdge }_{\text {max }}(F)=\max [\delta(F), 1-\varepsilon(F)]
\end{aligned}
$$

The new edge gradients were inspired by the standard discrete morphological gradient $F \oplus B-F \ominus B$, but to make the gradient operator more consistent with fuzzy set theory we replaced the difference between dilation and erosion with min (or max) of the dilation and the fuzzy complement of the 


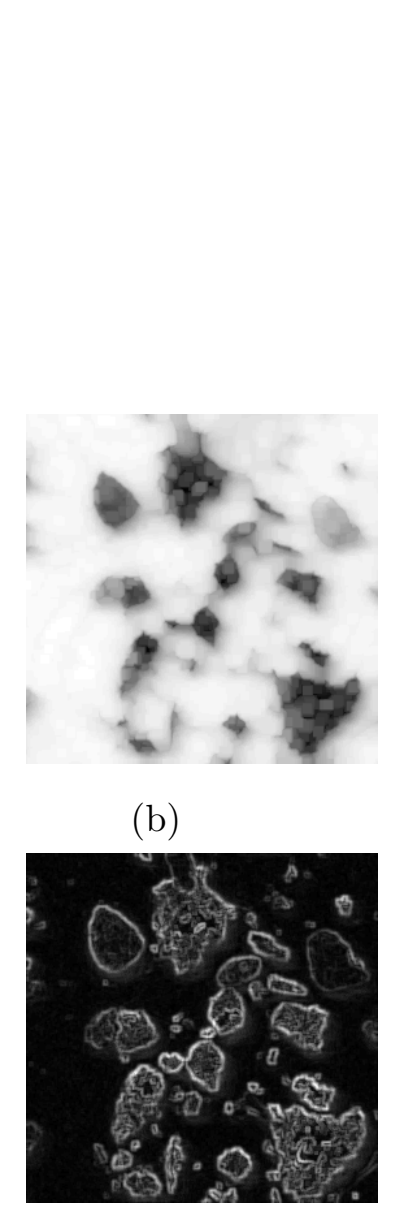

(f)

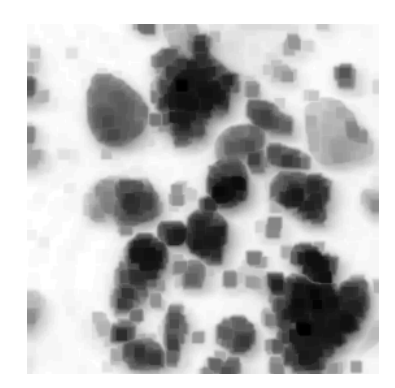

(c)

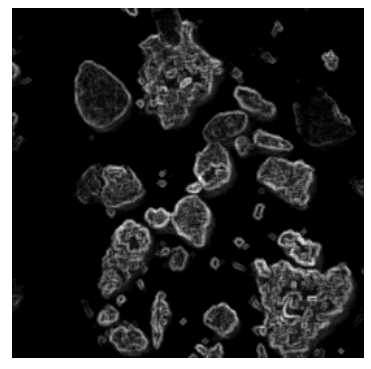

(g)

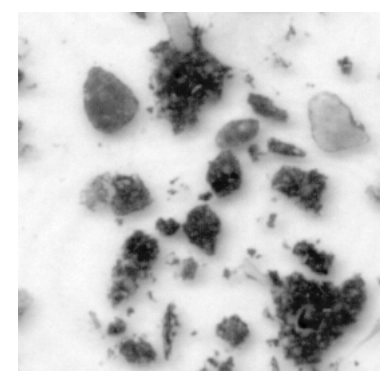

(a)

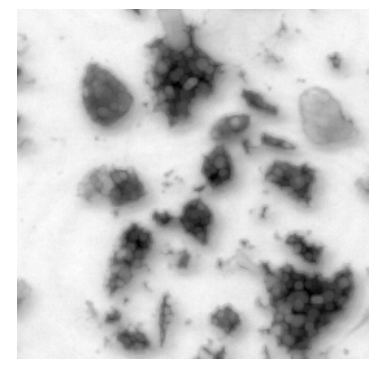

(d)

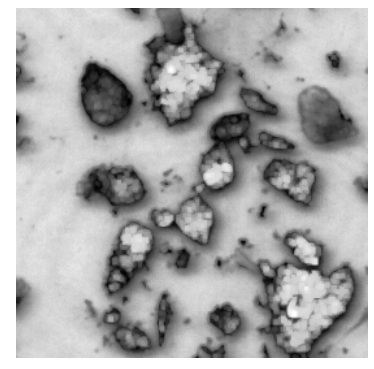

(h)

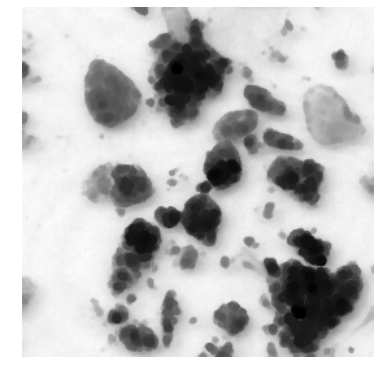

(e)

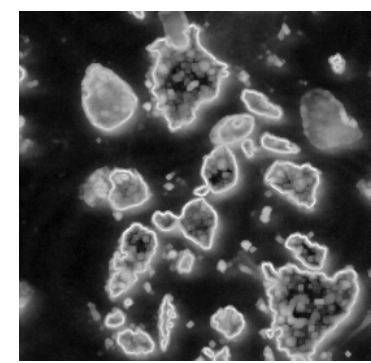

(i)

Figure 7: (a) Original image $F$. (b) Morphological flat dilation $F \oplus B$. (c) Morphological flat erosion $F \ominus B$. (d) Fuzzy dilation $\delta(F)$. (c) Fuzzy erosion $\varepsilon(F)$. (f) Morphological gradient $F \oplus B-F \ominus B$. (g) $\delta(F)-\varepsilon(F)$. (h) Fuzzy min gradient $\min [\delta(F), 1-\varepsilon(F)]$. (i) Fuzzy max gradient $\max [\delta(F), 1-$ $\varepsilon(F)]$. From Maragos et al. (2001).

erosion. As shown in Fig. 7, these new fuzzy gradient operators have a promising behavior since they yield cleaner and sharper edge peaks than the morphological gradient.

The power but also the difficulty in applying these lattice fuzzy operators to image analysis is the large variety of fuzzy norms and the absence of systematic ways in selecting them. As shown in Maragos et al. (2001, 2003), by experimenting with the type of fuzzy norm and the shape-size of the structuring function, it is possible to adapt the new fuzzy operators so that their performance has many promising aspects compared with the standard morphological operators.

\subsection{Matrix Representations of Image Operators on Finite-dimensional Weighted Minimax Vector Spaces}

In this section we shall work with a finite-dimensional function lattice $\mathcal{C}^{n}=\operatorname{Fun}(\{1,2, \ldots n\}, \mathcal{C})$, equipped with a scalar arithmetic of a clodum $\left(\mathcal{C}, \vee, \wedge, \star, \star^{\prime}\right)$ where $\mathcal{C} \subseteq \overline{\mathbb{R}}$. The underlying set $\mathcal{C}^{n}$ consists of all vectors with components from $\mathcal{C}$. The four scalar operations of $\mathcal{C}$ (and possibly the 
conjugation if it exists) are extended to vectors $\mathbf{x}, \mathbf{y} \in \mathcal{C}^{n}$ componentwise: ${ }^{20}$

$$
\begin{aligned}
& \{\mathbf{x} \vee \mathbf{y}\}_{i} \triangleq x_{i} \vee y_{i}, \quad i=1, \ldots, n \\
& \{\mathbf{x} \vee \mathbf{y}\}_{i} \triangleq x_{i} \vee y_{i}, \quad i=1, \ldots, n \\
& \{a \star \mathbf{x}\}_{i} \triangleq a \star x_{i}, \quad i=1, \ldots, n \\
& \left\{a \star^{\prime} \mathbf{x}\right\}_{i} \triangleq a \star^{\prime} x_{i}, \quad i=1, \ldots, n \\
& \left\{\mathbf{x}^{*}\right\}_{i} \triangleq x_{i}{ }^{*}, \quad i=1, \ldots, n
\end{aligned}
$$

Under these four operations, $\mathcal{C}^{n}$ becomes a complete weighted lattice $(C W L)$ vector space over $\mathcal{C}$. This nonlinear vector space is similar to the 'band spaces' of minimax algebra (Cuninghame-Green, 1979); however, we have endowed $\mathcal{C}^{n}$ with a richer algebraic structure, that of a complete weighted lattice.

Of interest are operators $\psi$ on $\mathcal{C}^{n}$ (i.e., vector transformations) that are increasing, i.e., $\mathbf{x} \leq \mathbf{y}$ implies $\psi(\mathbf{x}) \leq \psi(\mathbf{y})$. Elementary increasing operators are the scalar 'multiplications' of a vector by a scalar using the $\star$ or $\star^{\prime}$ operation componentwise. As done in Section 4.4.2 for general functions, we view these scalar 'multiplications' as vertical translations which shift the values of a vector $\mathbf{x}$ componentwise by combining them with a scalar $v$ via the operations $\star$ and $\star^{\prime}$ :

$$
\lambda_{v}(\mathbf{x}) \triangleq\left[v \star x_{i}\right], \quad \lambda_{v}^{\prime}(\mathbf{x}) \triangleq\left[v \star^{\prime} x_{i}\right]
$$

The set $\mathbb{L}$ all such vertical translations $\lambda_{v}$ forms a commutative monoid of vector dilations under composition, and so does the set of dual vertical translations $\lambda_{v}^{\prime}$ which are vector erosions.

If we define the elementary pulse vectors

$$
\mathbf{e}_{i} \triangleq\left[\mathcal{C}_{\mathrm{O}}, \ldots, \mathcal{C}_{\mathrm{O}}, \mathcal{C}_{\mathrm{id}}, \mathcal{C}_{\mathrm{O}}, \ldots, \mathcal{C}_{\mathrm{O}}\right]^{T}
$$

with a $\mathcal{C}_{\text {id }}$ value at the $i^{\text {th }}$ position and $\mathcal{C}_{\mathrm{O}}$ elsewhere, then the collection $\mathcal{B}=\left\{\mathbf{e}_{i}: i=1, \ldots, n\right\}$ is a finite upper basis for this CWL vector space. Specifically, each vector $\mathbf{x}=\left(x_{1}, \ldots, x_{n}\right)^{T}$ can be represented as a weighted maximum of basis vectors (or equivalently as a maximum of translated elementary vectors):

$$
\mathbf{x}=\bigvee_{i=1}^{n} x_{i} \star \mathbf{e}_{i}=\bigvee_{i=1}^{n} \tau_{x_{i}}\left(\mathbf{e}_{i}\right)
$$

Thus, the set $\mathcal{B}=\left\{\mathbf{e}_{i}: i=1, \ldots, n\right\}$ is a finite upper basis for this WMM vector space. For example, in the max-plus algebraic system where $\mathcal{C}=\left(\overline{\mathbb{R}}, \vee, \wedge,+,+^{\prime}\right)$, the basis vectors become:

$$
\mathbf{e}_{i} \triangleq[-\infty, \ldots,-\infty, 0,-\infty, \ldots,-\infty]^{T}
$$

and the representation of a vector from basis elements:

$$
\mathbf{x}=\bigvee_{i=1}^{n} x_{i}+\mathbf{e}_{i}
$$

A dual vector representation of (165) results from using a lower basis of dual elementary pulse vectors

$$
\mathbf{e}_{i}^{\prime} \triangleq\left[\mathcal{C}_{\mathrm{I}}, \ldots, \mathcal{C}_{\mathrm{I}}, \mathcal{C}_{\mathrm{id}}^{\prime}, \mathcal{C}_{\mathrm{I}}, \ldots, \mathcal{C}_{\mathrm{I}}\right]^{T}
$$

and form their weighted minimum of dual basis vectors:

$$
\mathbf{x}=\bigwedge_{i=1}^{n} x_{i} \star^{\prime} \mathbf{e}_{i}^{\prime}=\bigwedge_{i=1}^{n} \tau_{x_{i}}^{\prime}\left(\mathbf{e}_{i}^{\prime}\right)
$$

\footnotetext{
${ }^{20}$ Notation: If $\mathbf{M}=\left[m_{i j}\right]$ is a matrix, its $(i, j)$ th element is denoted as $\{\mathbf{M}\}_{i j}$ or simply $m_{i j}$. Similarly, if $\mathbf{x}=\left[x_{i}\right]$ is a vector, its $i^{\text {th }}$ element is denoted as $\{\mathbf{x}\}_{i}$ or simply $x_{i}$.
} 
Two very important types of increasing operators on this nonlinear vector space are the vector dilations $\delta$ and the vector erosions $\varepsilon$ which are defined as vector operators that distribute over any pointwise supremum and infimum of vectors, respectively:

$$
\delta\left(\bigvee_{i} \mathbf{x}_{i}\right)=\bigvee_{i} \delta\left(\mathbf{x}_{i}\right), \quad \varepsilon\left(\bigwedge_{i} \mathbf{x}_{i}\right)=\bigwedge_{i} \varepsilon\left(\mathbf{x}_{i}\right)
$$

Two special examples of vector dilation $\left(\delta_{\mathbf{M}}\right)$ and vector erosion $\left(\varepsilon_{\mathbf{M}}\right)$ are, respectively, the max'product' $⿴$ and min- $\star^{\prime}$ 'product' $\mathrm{⿴囗十}^{\prime}$ of a matrix $\mathbf{M}$ with an input vector:

$$
\delta_{\mathbf{M}}(\mathbf{x}) \triangleq \mathbf{M} \mathbb{} \mathbf{x}, \quad \varepsilon_{\mathbf{M}}(\mathbf{x}) \triangleq \mathbf{M} \mathbb{\Xi}^{\prime} \mathbf{x}
$$

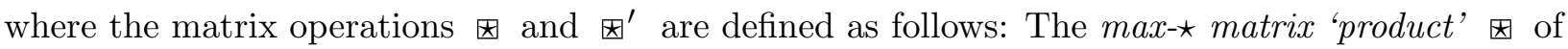
an arbitrary $m \times n$ matrix $\mathbf{A}=\left[a_{i j}\right]$ with an arbitrary $n \times p$ matrix $\mathbf{B}=\left[b_{i j}\right]$ is the $m \times p$ matrix $\mathbf{M}=\left[m_{i j}\right]$ defined as

$$
\mathbf{M}=\mathbf{A} \otimes \mathbf{B}, \quad m_{i j}=\bigvee_{k=1}^{n} a_{i k} \star b_{k j}
$$

Dually, their min- $\star^{\prime}$ matrix 'product' $\otimes^{\prime}$ is defined as

$$
\mathbf{M}=\mathbf{A} \mathbb{⿴ 囗 十}^{\prime} \mathbf{B}, \quad m_{i j}=\bigwedge_{k=1}^{n} a_{i k} \star^{\prime} b_{k j}
$$

Let us now combine on this nonlinear vector space the properties of being (vertical) translation invariant and increasing. In Section 4.4.2 we defined V-translation invariant dilations and erosions for general CWL function spaces. Now we specialize these definitions for the case of vector spaces. An operator $\delta$ on the CWL vector space is called a dilation V-translation invariant (DVI) operator iff

$$
\delta\left(\bigvee_{i} a_{i} \star \mathbf{x}_{i}\right)=\bigvee_{i} a_{i} \star \delta\left(\mathbf{x}_{i}\right), \quad \forall a_{i} \in \mathcal{C}, \mathbf{x}_{i} \in \mathcal{C}^{n}
$$

for any indexed vector collection. Dually, $\varepsilon$ is an erosion V-translation invariant (EVI) operator iff

$$
\varepsilon\left(\bigwedge_{i} a_{i} \star^{\prime} \mathbf{x}_{i}\right)=\bigwedge_{i} a_{i} \star^{\prime} \varepsilon\left(\mathbf{x}_{i}\right), \quad \forall a_{i} \in \mathcal{C}, \mathbf{x}_{i} \in \mathcal{C}^{n}
$$

Compare the above definitions with that of a linear operator $L$ on a linear vector space:

$$
L\left(\sum_{i} c_{i} \cdot \mathbf{x}_{i}\right)=\sum_{i} c_{i} \cdot L\left(\mathbf{x}_{i}\right)
$$

The following theorem establishes a one-to-one correspondence between DVI and EVI operators on this nonlinear vector space and the max-plus and min-plus matrix-based vector transformations, respectively.

Theorem 13 (a) (DVI): Any operator $\delta$ on $\mathcal{C}^{n}$ that obeys the max- $\star$ superposition of (172) can be represented as a matrix-based dilation $\delta_{\mathbf{M}}$ where $\mathbf{M}=\left[m_{i j}\right]$ with $m_{i j}=\left\{\delta\left(\mathbf{e}_{j}\right)\right\}_{i}$, and vice-versa. (b) (EVI): Any operator $\varepsilon$ on $\mathcal{C}^{n}$ that obeys the min- $\star^{\prime}$ superposition of (173) can be represented as a matrix-based erosion $\varepsilon_{\mathbf{M}}$ where $\mathbf{M}=\left[m_{i j}\right]$ with $m_{i j}=\left\{\varepsilon\left(\mathbf{e}_{j}^{\prime}\right)\right\}_{i}$, and vice-versa.

This theorem can be seen as a special case of Theorem 10. It establishes that any transformation $\psi$ on this nonlinear vector space that is a dilation (i.e. distributes over supremum) and V-translation invariant (i.e. commutes with the scalar operation $\star$ ) admits a matrix representation. Namely, it can be represented as a max-plus 'product' (170) of a matrix with the input vector. This characteristic matrix is formed by putting as columns the vectors $\psi\left(\mathbf{e}_{i}\right)$ where $\mathbf{e}_{i}, i=1, \ldots, n$ are the basis vectors. Dually, any EVI vector transformation admits a matrix representation; the details follow from above 
by replacing dilation with erosion, the scalar operation with $\star^{\prime}$, the matrix operation with the minplus 'product' (171), and the basis vectors with the dual pulse vectors $\mathbf{e}_{i}^{\prime}$. Compare the striking similarity between this pair of dual results with the result in Section 2.4.1 where we showed that any linear transformation over a finite-dimensional linear space admits a matrix representation as a regular matrix product of the input vector with a matrix whose columns are the transformations of the basis vectors.

\subsection{Elements from Max-plus Matrix Algebra and Spectral Analysis}

In this section we summarize some results of minimax algebra, on the eigenvalues and spectral analysis of max-plus matrix algebra.

Solving Max-Plus Equations: Consider the matrix $\mathbf{A} \in \overline{\mathbb{R}}^{m \times n}$ and the vector $\mathbf{b} \in \overline{\mathbb{R}}^{m}$. The set of solutions of

$$
\mathbf{A} \boxplus \mathbf{x}=\mathbf{b}
$$

over $\overline{\mathbb{R}}$ is either empty or forms a commutative semigroup under vector $\vee$. In Cuninghame-Green (1979) necessary and sufficient conditions are given for the existence and uniqueness of such solutions. One such result important for our analysis is given next, by using the conjugate matrix $\mathbf{A}^{*}$ where $\left\{\mathbf{A}^{*}\right\}_{i j}=-\{\mathbf{A}\}_{j i}$ for all $i, j$ :

$$
\mathbf{A}^{*} \triangleq-\mathbf{A}^{T}
$$

Theorem 14 (Cuninghame-Green, 1979).

Equation (175) has at least one solution iff $\mathbf{x}=\mathbf{A}^{*} \boxplus^{\prime} \mathbf{b}$ is a solution; and $\mathbf{x}=\mathbf{A}^{*} \boxplus^{\prime} \mathbf{b}$ is then the greatest solution.

An Optimization Problem in Max-Plus Algebra: In applications of max-plus algebra to scheduling, assume a problem where a vector $\mathbf{x}$ represents start times, a vector $\mathbf{b}$ represents finish times and a matrix A represents processing delays. Then, assuming that (175) does not have an exact solution, it is possible to find the optimum $\mathbf{x}$ such that we minimize a norm of the earliness subject to zero lateness. This optimum will be the solution of the following constrained minimization problem:

$$
\begin{gathered}
\text { Minimize }\|\mathbf{b}-\mathbf{A} \boxplus \mathbf{x}\| \\
\text { subject to } \mathbf{A} \boxplus \mathbf{x} \leq \mathbf{b}
\end{gathered}
$$

where the norm $\|\cdot\|$ is either the $\ell_{\infty}$ or the $\ell_{1}$ norm.

Theorem 15 (Cuninghame-Green, 1979).

The solution to the optimization problem (177) is

$$
\mathbf{x}=\mathbf{A}^{*} \boxplus^{\prime} \mathbf{b}
$$

Vector Independence: Eq. (175) can also be written as

$$
\bigvee_{j=1}^{n} \mathbf{a}(j)+x_{j}=\mathbf{b}
$$

where $\mathbf{a}(j) \in \overline{\mathbb{R}}^{m}, j=1, \ldots, n$, are the $n$ consecutive columns of $\mathbf{A}$. If $x_{j}>-\infty \forall j$, we say that $\mathbf{b}$ is max-plus dependent ${ }^{21}$ on all the $\mathbf{a}(j), \ldots, \mathbf{a}(n)$. By negation of max-plus dependence, the vectors $\mathbf{a}(j), \ldots, \mathbf{a}(n)$ are called max-plus independent iff none of them is max-plus dependent on the others. A stronger and perhaps more useful type of independence is the following. The vectors $\mathbf{a}(j), \ldots$, $\mathbf{a}(n)$ are called strongly max-plus independent (SMI) iff there exists a finite $\mathbf{b} \in \mathbb{R}^{m}$ that has a unique expression of the form (179) with all $x_{j}$ finite and the max of each row and column of $\mathbf{A}$ is a finite real.

\footnotetext{
${ }^{21}$ What we call here 'max-plus (in)dependence', Cuninghame-Green (1979) calls it 'linear (in)dependence'. We changed the terminology because it may be confused with the corresponding concept for linear operators.
} 
Theorem 16 (Cuninghame-Green, 1979).

The vectors $\mathbf{a}(j), \ldots, \mathbf{a}(n)$ are SMI iff there exists a finite $\mathbf{b} \in \mathbb{R}^{m}$ such that (175) is uniquely soluble.

Matrix Rank: If we can find $r$ columns of $\mathbf{A}(1 \leq r \leq n)$, but no more, that are SMI, then $\mathbf{A}$ is said to have column-rank equal to $r$.

Graph of a Matrix: Each square matrix $\mathbf{A}=\left[a_{i j}\right] \in \overline{\mathbb{R}}^{n \times n}$ can be represented by a directed weighted graph $\operatorname{Gr}(\mathbf{A})$ that has $n$ nodes, is strongly complete, i.e., for each pair of nodes there is a corresponding directed graph branch (arc) joining them, and the weight of each arc joining a pair of nodes $(i, j)$ is equal to $a_{i j}$. Consider a path on the graph, i.e., a sequence of nodes $P=\left(i_{0}, i_{1}, \ldots, i_{t}\right)$; its length $L(P)$ and weight $W(P)$ are defined, respectively, by:

$$
L(P) \triangleq \# \operatorname{arcs} \text { on } P=t, \quad W(P) \triangleq a_{i_{0} i_{1}}+\ldots+a_{i_{t-1} i_{t}}
$$

A path is called a circuit if $i_{0}=i_{t}$; the circuit is elementary if the nodes $i_{0}, \ldots, i_{t-1}$ are pairwise distinct. For any circuit $P$ we can define its average weight by $W(P) / L(P)$. Let

$$
\lambda(\mathbf{A}) \triangleq \bigvee_{\text {all circuits } P \text { of } \mathbf{A}} \frac{W(P)}{L(P)}
$$

be the maximum average circuit weight in $\operatorname{Gr}(\mathbf{A})$. Since $\operatorname{Gr}(\mathbf{A})$ has $n$ nodes, only elementary circuits (with lenth $\leq n$ ) need be considered in (180). There is also at least one circuit whose average weight coincides with the maximum value (180); such a circuit is called critical.

Definite and Metric Matrices: A matrix A is called definite if every circuit in its graph has weight $\leq 0$ and at least one such circuit has weight $=0$. The metric matrix generated by a matrix A is defined by

$$
\Gamma(\mathbf{A}) \triangleq \mathbf{A} \vee \mathbf{A}^{(2)} \vee \ldots \vee \mathbf{A}^{(n)}
$$

Eigenvalues, Eigenvectors: Given a square matrix $\mathbf{A}=\left[a_{i j}\right] \in \overline{\mathbb{R}}^{n \times n}$, we say that $\mathbf{x} \in \overline{\mathbb{R}}^{n}$ is an eigenvector of $\mathbf{A}$ and $\lambda \in \overline{\mathbb{R}}$ a corresponding eigenvalue of $\mathbf{A}$ if

$$
\mathbf{A} \boxplus \mathbf{x}=\lambda+\mathbf{x}
$$

If we can find finite $\lambda$ and $\mathbf{x}$ satisfying (182), then we say that the eigenproblem is finitely soluble for $\mathbf{A}$. If $\mathbf{A}$ is definite, its associated graph $\operatorname{Gr}(\mathbf{A})$ contains at least one circuit with zero weight. An eigennode is any node on such a circuit.

Theorem 17 (Cuninghame-Green, 1979).

Let $\mathbf{A}$ be definite. Then:

(a) $j$ is an eigennode of $\operatorname{Gr}(\mathbf{A})$ iff $\{\Gamma(\mathbf{A})\}_{j j}=0$.

(b) If $j$ is an eigennode of $\operatorname{Gr}(\mathbf{A})$, then the $j$ th column of $\Gamma(\mathbf{A})$ is an eigenvector of $\mathbf{A}$ whose corresponding eigenvalue is zero.

Thus, columns of $\Gamma(\mathbf{A})$ that correspond to eigennodes provide eigenvectors for $\mathbf{A}$, which are called fundamental eigenvectors. Two such eigenvectors are called equivalent if their corresponding eigennodes belong to the same critical circuit. Max-plus combinations of non-equivalent fundamental eigenvectors generate the eigenspace of $\mathbf{A}$, whose elements are eigenvectors of $\mathbf{A}$ with corresponding eigenvalue $=0$.

Theorem 18 (Cuninghame-Green, 1979).

(a) If the eigenproblem for $\mathbf{A}$ is finitely soluble, then every finite eigenvector has the same unique finite eigenvalue, called the principal eigenvalue, which is equal to the maximum average circuit weight of $\mathbf{A}$ defined in (180). All finite eigenvectors of $\mathbf{A}$ lie in the eigenspace of the definite matrix $\mathbf{A}-\lambda(\mathbf{A})$. The non-equivalent fundamental eigenvectors which generate this space are SMI.

(b) The eigenproblem for $\mathbf{A}$ is finitely soluble iff $\lambda(\mathbf{A})$ is finite and $\phi(\mathbf{A}-\lambda(\mathbf{A}))$ has rows and columns whose maxima are finite, where $\phi(\mathbf{A}-\lambda(\mathbf{A}))$ is any matrix whose columns form a maximal set of non-equivalent fundamental eigenvectors for the definite matrix $\mathbf{A}-\lambda(\mathbf{A})$.

(c) If $\mathbf{A}$ is finite, then the eigenproblem for $\mathbf{A}$ is finitely soluble. 


\section{Kernel and Basis Representations of Operators on Lattices}

Consider $m$-dimensional binary images represented by subsets of $\mathbb{E}^{m}$, where $\mathbb{E}^{m}$ is equal to $\mathbb{R}^{m}$ or $\mathbb{Z}^{m}$. This section begins with a representation theory unifying all set operators $\Psi$ on $\mathcal{P}\left(\mathbb{E}^{m}\right)$ that are translation-invariant (TI), i.e.

$$
\Psi\left(X_{+\mathbf{z}}\right)=\Psi(X)_{+\mathbf{z}}, \quad \forall X \subseteq \mathbb{E}^{m}, \mathbf{z} \in \mathbb{E}^{m}
$$

TI operators have the exceedingly desirable property that the result of an image transformation is independent of the exact location of the image object.

(1) The first main result refers to a very broad class of translation-invariant operators that have two additional properties of being increasing and monotonically continuous. It will be shown that such operators can be represented exactly as a minimal union (resp. intersection) of morphological erosions (resp. dilations). These results will then be extended to increasing image and signal operators $\psi$ operating on $\operatorname{Fun}\left(\mathbb{E}^{m}, \overline{\mathbb{R}}\right)$ that are translation-invariant, i.e.

$$
\psi\left(f_{\mathbf{h}, v}\right)=\psi(f)_{\mathbf{h}, v}, \quad \forall f_{\mathbf{h}, v}(\mathbf{x})=f(\mathbf{x}-\mathbf{h})+v, \quad \mathbf{h} \in \mathbb{E}^{m}, v \in \overline{\mathbb{R}}
$$

Again, such operators will be represented as suprema (infima) of flat or weighted Minkowski function erosions (dilations) by some basis elements that uniquely characterize the operator. All these aforementioned representations establish as building blocks of all translation-invariant set or signal operators the Minkowski erosions and dilations, which are essentially infimal and supremal convolutions.

The above representation theory is also extended to several other categories of operators: (2) TI Non-increasing set operators: in this case the representations will be unions (or intersections) of non-monotone operators by set intervals in some basis. The building blocks are operations that are closely related to hit-miss transformations for shape detection. (3) Increasing Spatially-Varying operators: here we find representations as unions of spatially-varying erosions by structuring element maps in a collection that uniquely characterizes the operator. (4) Operators on complete weighted lattices invariant with respect to generalized translations. (5) TI set openings: here we examine the representation of general TI lattice openings both as unions of Minkowski openings by some of their fixed sets as well as unions of erosions. We illustrate the above cases with several examples.

In terms of terminology, whenever we mention simply 'translation-invariant (in short, TI)' operators, we shall mean operators that commute with Euclidean translations, as in (183),(184).

\subsection{Kernel Representation of TI Increasing Set Operators}

Consider set operators on $\mathcal{P}\left(\mathbb{E}^{m}\right)$. The kernel of a TI operator $\Psi$ is defined as the following collection of input sets:

$$
\operatorname{Ker}(\Psi) \triangleq\left\{A \subseteq \mathbb{E}^{m}: \mathbf{0} \in \Psi(A)\right\},
$$

where $\mathbf{0}$ denotes the zero vector of $\mathbb{E}^{m}$.

Example 10 (Kernel of Erosion and Dilation):

The kernel of the set erosion operator $\Psi(X)=X \ominus B$ is

$$
\mathcal{K} e_{B} \triangleq \operatorname{Ker}(X \mapsto X \ominus B)=\{A: A \supseteq B\},
$$

where $\operatorname{Ker}(X \mapsto \Psi(X))$ denotes $\operatorname{Ker}(\Psi)$. The kernel of the set dilation $\Psi(X)=X \oplus B$ is

$$
\mathcal{K} d_{B} \triangleq \operatorname{Ker}(X \mapsto X \oplus B)=\left\{A: A \cap B^{s} \neq \varnothing\right\} .
$$

A fundamental kernel property is that we can uniquely synthesize a TI operator $\Psi$ if we know its kernel as follows:

$$
\Psi(X)=\left\{\mathbf{z} \in \mathbb{E}^{m}: X_{-\mathbf{z}} \in \operatorname{Ker}(\Psi)\right\}, \quad X \subseteq \mathbb{E}^{m} .
$$

Other kernel properties include the following. If $\left\{\Psi_{i}: i \in I\right\}$ is an indexed family of TI set operators, it is simple to prove that intersection, union and ordering of operators induces the same operations for their kernels, as stated next. 
Proposition 17 (Properties of Kernels).

(a) $\operatorname{Ker}\left(\bigcap_{i \in I} \Psi_{i}\right)=\bigcap_{i \in I} \operatorname{Ker}\left(\Psi_{i}\right)$.

(b) $\operatorname{Ker}\left(\bigcup_{i \in I} \Psi_{i}\right)=\bigcup_{i \in I} \operatorname{Ker}\left(\Psi_{i}\right)$.

(c) $\Psi_{1} \subseteq \Psi_{2} \Longleftrightarrow \operatorname{Ker}\left(\Psi_{1}\right) \subseteq \operatorname{Ker}\left(\Psi_{2}\right)$.

(d) The complete lattice of TI operators on $\mathcal{P}\left(\mathbb{E}^{m}\right)$ is isomorphic to the lattice $\mathcal{P}\left(\mathcal{P}\left(\mathbb{E}^{m}\right)\right)$ ) of their kernel collections.

(e) If the TI set operator $\Psi$ is increasing and $\varnothing \neq X \in \operatorname{Ker}(\Psi)$, then $Y \in \operatorname{Ker}(\Psi)$ for all $Y \supseteq X$.

Assume henceforth that we deal only with TI set operators $\Psi$ that are nondegenerate: i.e.,

$$
\Psi(\varnothing)=\varnothing \quad, \quad \Psi\left(\mathbb{E}^{m}\right)=\mathbb{E}^{m},
$$

and that $\operatorname{Ker}(\Psi)$ contains more ${ }^{22}$ elements than the single set $\mathbb{E}^{m}$.

Let $\Psi$ be a TI increasing set operator. The dual (or negative) set operator of $\Psi$ is defined by

$$
\Psi^{*}(A) \triangleq\left[\Psi\left(A^{c}\right)\right]^{c}
$$

Obviously, $\Psi^{*}$ is TI and increasing if and only if $\Psi$ is TI and increasing, respectively. The kernel of any TI increasing set operator has the following remarkable property:

Theorem 19 (Matheron, 1975). Let $\Psi: \mathcal{P}\left(\mathbb{E}^{m}\right) \rightarrow \mathcal{P}\left(\mathbb{E}^{m}\right)$ be a TI increasing set operator. Then

$$
\Psi(X)=\bigcup_{A \in \operatorname{Ker}(\Psi)} X \ominus A=\bigcap_{B \in \operatorname{Ker}\left(\Psi^{*}\right)} X \oplus B^{s} .
$$

\subsection{Basis Representation of TI Increasing Set Operators}

Theorem 19 a may be theoretically interesting but has no direct practical importance, because it requires an infinite number of erosions to implement an increasing TI operator, since the kernel of such a operator has an infinite number of elements due to property 17(e). This motivated Maragos (1985) to introduce the concept of the basis of such operators, which is defined as the collection of minimal kernel elements. If the basis is nonempty, then we may be able to exactly represent an operator as a minimal (possibly finite) union of erosions using just the basis elements.

The kernel $\operatorname{Ker}(\Psi)$ of a set operator $\Psi$ is a partially ordered set under set inclusion. A kernel set-element is minimal in $(\operatorname{Ker}(\Psi), \subseteq)$ if and only if it is not preceded (with respect to $\subseteq$ ) by any other kernel set. If $\Psi$ is also increasing, and $M \in \operatorname{Ker}(\Psi)$, then $\{A: A \supseteq M\} \subseteq \operatorname{Ker}(\Psi)$. In addition, $X \ominus A \subseteq X \ominus M$ for any set $X$ and $A \supseteq M$. Thus, in representing $\Psi$ as a union of erosions, the erosion by $M$ contains the erosions by any other kernel set larger than $M$, and, hence, it is the only one needed. The morphological ${ }^{23}$ basis of any TI set operator $\Psi$ is defined as the collection of its minimal kernel sets, denoted as

$$
\operatorname{Bas}(\Psi) \triangleq\{M \in \operatorname{Ker}(\Psi):[A \in \operatorname{Ker}(\Psi) \text { and } A \subseteq M] \Longrightarrow A=M\}
$$

At this point, two fundamental questions naturally arise: Does the basis exists? Can we represent the operator only by its basis? In what follows we will answer both of these questions affirmatively. Toward this goal we have to restrict the general space of signals. Thus, instead of the most general set class $\mathcal{P}\left(\mathbb{E}^{m}\right)$, now we select for image representation the class $\mathcal{F}\left(\mathbb{E}^{m}\right)$ of all closed subsets of $\mathbb{E}^{m}$. This is a natural compromise if we assume that each image object contains its boundary. This is a restriction only if $\mathbb{E}^{m}=\mathbb{R}^{m}$. Discrete sets, i.e. subsets of $\mathbb{Z}^{m}$, are all closed.

\footnotetext{
${ }^{22}$ This assumption on the kernel (i.e. that it contains more elements than just $\mathbb{E}^{m}$ ), in addition to (189), practically excludes from our discussion the greatest dilation operator which maps $\varnothing$ to $\varnothing$ and every other input set to $\mathbb{E}^{m}$.

${ }^{23}$ If there is possibility of confusion with other concepts of 'basis', we shall call (191) the 'morphological basis'; otherwise, we shall simply call it the 'basis' of the TI increasing operator.
} 
A sufficient condition to prove the existence of a basis for increasing TI operators is to assume that they are also upper semi-continuous (u.s.c.). If an operator is increasing, then there is an easy way to check its upper semi-continuity: ${ }^{24}$ Let $\left(X_{n}\right)$ be any decreasing sequence of sets that converges monotonically to a limit set $X$, i.e., $X_{n+1} \subseteq X_{n} \forall n$ and $X=\bigcap_{n} X_{n}$; we denote this by $X_{n} \downarrow X$. An increasing set operator $\Psi$ on $\mathcal{F}\left(\mathbb{E}^{m}\right)$ is upper semi-continuous if and only if $X_{n} \downarrow X$ implies that $\Psi\left(X_{n}\right) \downarrow \Psi(X)$. Upper semi-continuity amounts to requiring operators to be insensitive to fine details in the signal, since a physical resolution limit cannot be avoided. This, together with the already discussed importance of increasingness and translation-invariance, makes TI increasing upper semi-continuous operators an important class of image and signal operators. Such operators are "digitalizable", meaning that the transition between transforming continuous and discrete image objects satisfies a continuity condition (Serra, 1982). Further, they play a central role in our discussion since the basis of a TI increasing set or signal operator exists if the operator is upper semi-continuous, as explained next.

Theorem 20 (Maragos, 1985, 1989a).

Let $\Psi: \mathcal{F}\left(\mathbb{E}^{m}\right) \rightarrow \mathcal{F}\left(\mathbb{E}^{m}\right)$ be a TI, increasing and upper semi-continuous set operator. Then, the kernel of $\Psi$ has a minimal element. Further, for any $A \in \operatorname{Ker}(\Psi)$, there exists a minimal kernel set $M \in \operatorname{Bas}(\Psi)$ such that $A \supseteq M$.

Theorem 20 establishes that, given a TI, increasing and upper semi-continuous set operator $\Psi$, we don't need its whole kernel $\mathcal{K}$ to represent it as a union of erosions $\Psi(X)=\bigcup_{A \in \mathcal{K}} X \ominus A$, but we only need a minimal subcollection, its basis $\mathcal{B}$. Namely, for each $A \in \mathcal{K}$ there is a $M \in \mathcal{B}$ such that $M \subseteq A$; thus the erosion $X \ominus A$ is not needed because it is contained in $X \ominus M$. Hence, $\Psi(X)=\bigcup_{M \in \mathcal{B}} X \ominus M$. To find a dual basis representation involving dilations, we restrict the domain on which binary images are defined to be $\mathbb{E}^{m}=\mathbb{Z}^{m}$. Next is the first theorem for representation by minimal elements.

Theorem 21 (Maragos, 1985, 1989a).

(a) Let $\Psi: \mathcal{F}\left(\mathbb{R}^{m}\right) \rightarrow \mathcal{F}\left(\mathbb{R}^{m}\right)$ be a TI, increasing and upper semi-continuous set operator. Then $\Psi$ can be represented exactly as the union of Minkowski erosions by all its basis sets; i.e.,

$$
\Psi(X)=\bigcup_{M \in \operatorname{Bas}(\Psi)} X \ominus M .
$$

(b) Let $\Psi: \mathcal{P}\left(\mathbb{Z}^{m}\right) \rightarrow \mathcal{P}\left(\mathbb{Z}^{m}\right)$ be a discrete $T I$, increasing and upper semi-continuous set operator. Then, if the dual operator $\Psi^{*}$ is upper semi-continuous, $\Psi$ can be represented as the union of Minkowski erosions by all its basis sets, and also as the intersection of Minkowski dilations by all the reflected basis sets of $\Psi^{*}$; i.e.,

$$
\Psi(X)=\bigcup_{M \in \operatorname{Bas}(\Psi)} X \ominus M=\bigcap_{N \in \operatorname{Bas}\left(\Psi^{*}\right)} X \oplus N^{s} .
$$

Since we consider only nondegenerate operators, the basis is a proper subset of the kernel. Hence, there is a proper subset $M$ of $\mathbb{E}^{m}$ belonging to the basis. Then, all the (infinite in number) sets $X$ such that $M \subseteq X \subseteq \mathbb{E}^{m}$ belong to the kernel but not to the basis. Thus, Theorem 21, compared with Theorem 19, realizes the operator by infinitely reducing the number of required erosions (or dilations).

The previous result establishes the erosions and dilations as the building elements of any TI, increasing and u.s.c. operator. The class of such operators is closed under parallel and serial combinations of them.

Proposition 18 Any finite union, intersection, and composition of TI, increasing and u.s.c. operators is also TI, increasing and u.s.c.

\footnotetext{
${ }^{24}$ Here upper semi-continuity is meant with respect to the hit-miss topology of the space $\mathcal{F}\left(\mathbb{E}^{m}\right)$ of the closed subsets; for definitions see Section 3.7.1.
} 


\subsection{Examples of Set Operator Bases}

One technique to find the basis of a set operator $\Psi$ is based on the fact that its minimal kernel elements are the smallest (with respect to $\subseteq$ ) solutions $X$ of the set inequality $\mathbf{0} \in \Psi(X)$. Finding, however, these solutions depends upon the specific operator, as the following examples indicate.

\subsubsection{Morphological Set Operators}

Example 11 Minkowski Erosion: Consider the set erosion $\mathcal{E}(X)=X \ominus A$ by a fixed set $A$. Then $\mathbf{0} \in X \ominus A \Longleftrightarrow A \subseteq X$; the smallest $X \supseteq A$ is $A$. Hence, the erosion basis is

$$
\operatorname{Bas}(X \mapsto X \ominus A)=\{A\}
$$

Example 12 Minkowski Dilation: Consider the set dilation $\Delta(X)=X \oplus A$. Clearly, $\mathbf{0} \in$ $\Delta(X) \Longleftrightarrow X \cap A^{s} \neq \varnothing \Longleftrightarrow-\mathbf{a} \in X$ for some $\mathbf{a} \in A$. Hence, the dilation basis is

$$
\operatorname{Bas}(X \mapsto X \oplus A)=\{\{-\mathbf{a}\}: \mathbf{a} \in A\}
$$

Example 13 Minkowski Opening: Let $\Gamma(X)=X \circ A=(X \ominus A) \oplus A$. Since

$$
\begin{gathered}
X \circ A=\bigcup_{\mathbf{a} \in A}(X \ominus A)_{+\mathbf{a}}=\bigcup_{\mathbf{a} \in A} X \ominus A_{-\mathbf{a}}=\bigcup_{\mathbf{a} \in A}\left\{\mathbf{z}: A_{-\mathbf{a}+\mathbf{z}} \subseteq X\right\} \\
\mathbf{0} \in X \circ A \Longleftrightarrow A_{-\mathbf{a}} \subseteq X \text { for some } \mathbf{a} \in A
\end{gathered}
$$

Thus, the kernel of the opening is

$$
\operatorname{Ker}(X \mapsto X \circ A)=\left\{X: X \supseteq A_{-\mathbf{a}} \text { for some } \mathbf{a} \in A\right\}
$$

Hence, the basis of the set opening is

$$
\operatorname{Bas}(X \mapsto X \circ A)=\left\{A_{-\mathbf{a}}: \mathbf{a} \in A\right\}
$$

Example 14 Minkowski Closing: Let $\Phi(X)=X \bullet A=(X \oplus A) \ominus A$, where $A$ is compact. Since

$$
X \bullet A=\bigcap_{\mathbf{a} \in A} X \oplus A_{-\mathbf{a}}
$$

the kernel is

$$
\operatorname{Ker}(X \mapsto X \bullet A)=\left\{X: X \cap\left(A^{s}\right)_{+\mathbf{a}} \neq \varnothing \quad \forall \mathbf{a} \in A\right\}
$$

In this case we cannot find explicitly the basis elements, but we can find a fixed upper bound. That is, let $G \in \operatorname{Ker}(\Phi)$ and define

$$
H=\bigcup_{\mathbf{a} \in A} G \cap\left(A^{s}\right)_{+\mathbf{a}}
$$

Obviously, $G \supseteq H$ and $H \in \operatorname{Ker}(\Phi)$. By Theorem 20, the kernel sets $G$ and $H$ contain a minimal element $M$. Then,

$$
M \subseteq H \subseteq \bigcup_{\mathbf{a} \in A}\left(A^{s}\right)_{+\mathbf{a}}=A \oplus A^{s}
$$

Hence, the basis of the set closing is

$$
\operatorname{Bas}(X \mapsto X \bullet A)=\left\{M \subseteq A \oplus A^{s}: \mathbf{0} \in M \bullet A \text { and } M \text { is minimal }\right\}
$$


Thus, the basis of discrete set erosions, dilations, openings, and closings by a finite set $A$ is finite. Specifically, the erosion has only one basis set, the dilation $n$, and the opening $n$ basis sets; the number of elements in the basis of the closing is smaller than $\left|\mathcal{P}\left(A \oplus A^{s}\right)\right|$, where $|\cdot|$ denotes set cardinality.

Next we focus on the basis of the opening and its dual closing. Since $X \circ A=\left(X^{c} \bullet A^{s}\right)^{c}$, the dual set operator of the opening by $A$ is the closing by $A^{s}$. Thus, the last two examples imply the following representation of a Minkowski set opening:

$$
X \circ A=\bigcup_{\mathbf{a} \in A} X \ominus A_{-\mathbf{a}}=\bigcap_{M \in \operatorname{Bas}\left(Y \mapsto Y \bullet A^{s}\right)} X \oplus M^{s} .
$$

For 2D structuring sets $A$, the basis of the opening is relatively easy to find by using (198), whereas the minimal elements of the closing require some search procedure. In general, we observe that for both the opening and the closing by $A$, their basis sets are subsets of the finite window $W=A \oplus A^{s}$. Interesting geometrical structures in the basis of a discrete closing by $2 \mathrm{D}$ sets have been found by Svalbe (1991), who has also found a geometric connection between the basis sets of a closing and the complete minimal representation of logic functions.

Example 15 . Consider the opening and closing on $\mathbb{Z}^{2}$ by the 4-point discrete square set $A=$ $\{(0,0),(0,1),(1,0),(1,1)\}$. As shown in Fig. 8, the basis of the opening by $A$ has only four 4 -point sets, whereas the basis of the closing by $A$ has twelve sets: $\{(0,0)\}$, two 2-point sets, eight 3-point sets, and one 4-point set.

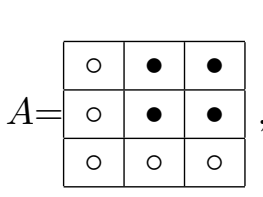

Basis of Opening

\begin{tabular}{|c|c|c|c|c|c|c|c|c|c|}
\hline 0 & 0 & 0 & & • & $\bullet$ & ? & ( & 0 & . \\
\hline 0 & ? & 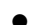 & & 9 & 0 & 0 & 9 & 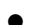 & 0 \\
\hline 0 & • & $\bullet$ & & 0 & 0 & 0 & $\bullet$ & $\bullet$ & 0 \\
\hline
\end{tabular}
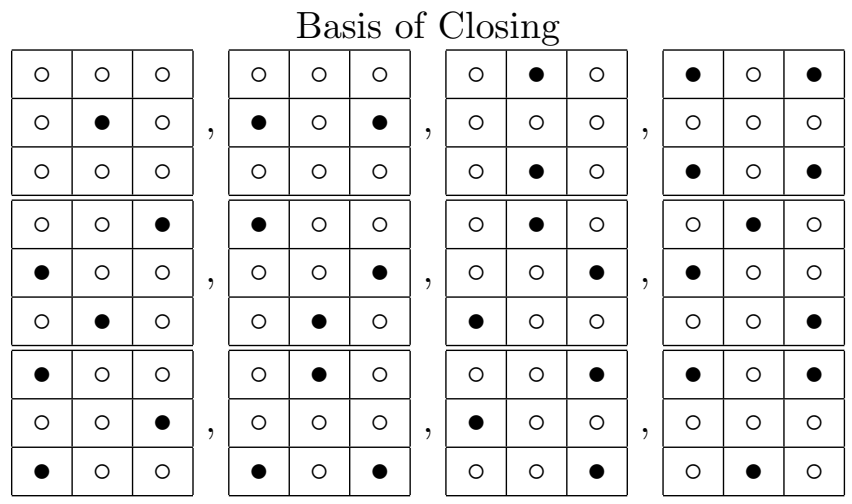

Figure 8: Basis sets of the set opening and closing by the $2 \times 2$-pixel square $A$. In both cases, all the basis sets are subsets of the $3 \times 3$-pixel square $W=A \oplus A^{s}$. Points denoted by $\bullet$ belong to basis sets and to $W$; points denoted by $\circ$ belong to $W$ but not to basis sets.

\subsubsection{Median and Rank Filters for Sets}

Consider discrete sets $X \subseteq \mathbb{Z}^{m}$ and a finite window $W \subseteq \mathbb{Z}^{m}$ with cardinality $n=|W|$ points. The $k$-th rank set operator by $W$ is

$$
\Xi(X)=\mathcal{R}_{W, k}(X) \triangleq\left\{\mathbf{x} \in \mathbb{Z}^{m}:\left|X \cap W_{+\mathbf{x}}\right| \geq k\right\}
$$

The rank operators contain as special cases the dilation (for $k=1$ ) and the erosion (for $k=n$ ), since

$$
\mathcal{R}_{W, 1}(X)=X \oplus W^{s}, \quad \mathcal{R}_{W,|W|}(X)=X \ominus W
$$


If $n$ is odd, the rank for $k=(n+1) / 2$ is called median and denoted by $\operatorname{med}_{W}(\cdot)$.

The basis of the $k$-th rank operator is

$$
\operatorname{Bas}\left(X \mapsto \mathcal{R}_{W, k}(X)=\{M \subseteq W:|M|=k\},\right.
$$

and has $\frac{n !}{k !(n-k) !}$ elements. For example, let $m=2$ and $W=\{(0,0),(0,1),(1,0),(-1,0),(0,-1)\}$ be the 5-pixel discrete rhombus centered at the origin of $\mathbb{Z}^{2}$. Then the basis of the set median by $W$ has ten elements which are all the 3-pixel subsets of $W$; see Fig. 9.
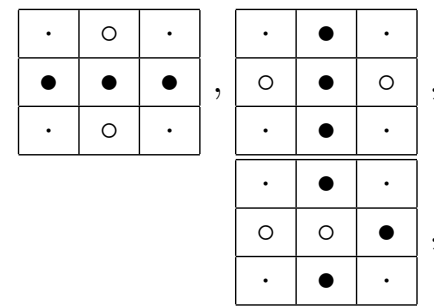
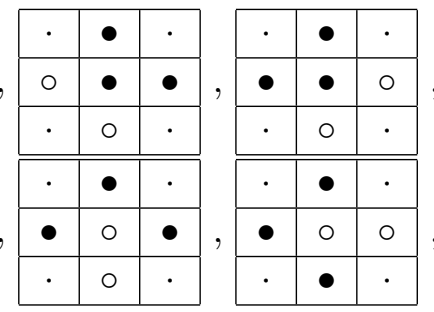
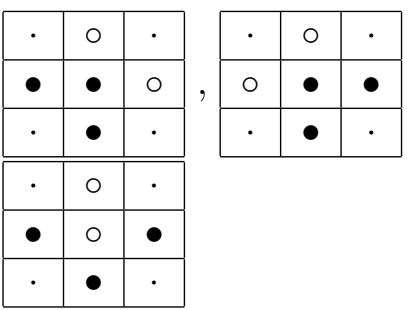

Figure 9: Basis sets of the set median by the 5-pixel rhombus window $W$. Points denoted by $\bullet$ belong to basis sets and to $W$; points denoted by o belong to $W$ but not to basis sets. Points denoted by - do not belong to $W$.

The dual set operator of $\Xi$ is

$$
\Xi^{*}(X)=\left(\Xi\left(X^{c}\right)\right)^{c}=\left\{\mathbf{x} \in \mathbb{Z}^{m}:\left|X \cap W_{+\mathbf{x}}\right| \geq n-k+1\right\}
$$

which is the $(n-k+1)^{t h}$ rank by $W$.

For each rank set operator, the basis representation theorem yields the following alternative expression:

$$
\mathcal{R}_{W, k}(X)=\bigcup_{\substack{A \subseteq W \\|A|=k}} X \ominus A=\bigcap_{\substack{B \subseteq W \\|B|=n-k+1}} X \oplus B^{s} .
$$

Thus, any median and rank set operator can be expressed via a closed formula involving only union (resp. intersection) of erosions (resp. dilations) by pre-specified subsets of the window without requiring any sorting or counting of pixels inside the moving window.

\subsubsection{Window Transformations for Shape Detection and Locally-defined Set Opera- tors}

For the purpose of shape detection, Crimmins and Brown (1985) defined the following type of transformation: Let $W \subseteq \mathbb{Z}^{m}$ be some finite window. A discrete set operator $\Psi$ is called a window transformation if there exists a shape collection $\mathcal{T} \subseteq \mathcal{P}(W)$ such that

$$
\Psi(X)=\left\{\mathbf{p} \in \mathbb{Z}^{m}: W \cap X_{-\mathbf{p}} \in \mathcal{T}\right\}, X \subseteq \mathbb{Z}^{m} .
$$

The patterns in $\mathcal{T}$ are exactly the shapes or templates or objects that the window operator can detect. Given a window operator $\Psi$ we can find its defining shape collection as follows:

$$
\mathcal{T}(\Psi)=\{A \subseteq W: \mathbf{0} \in \Psi(A)\}
$$

Thus, there is a one-to-one correspondence between the window operator and its shape collection.

Let $A$ be a fixed finite set and let $W$ be another finite set containing $A$ such that the set difference $W \backslash A$ serves as a border (narrow ring) around $A$. Then the hit-miss transformation $(X \ominus A) \cap\left[X^{c} \ominus(W \backslash A)\right]$ provides the set of points $\mathbf{p}$ at which $A$ "fits exactly" inside an input set $X$; hence, it acts as a shape detection operator. Essentially, it is an erosion-based matched filter. The next result shows that this special case of a hit-miss operator is the prototype for any window transformation. 
Theorem 22 (Crimmins and Brown, 1985).

Let $\Psi: \mathcal{P}\left(\mathbb{Z}^{m}\right) \rightarrow \mathcal{P}\left(\mathbb{Z}^{m}\right)$ be a window transformation with window $W$. Then

$$
\Psi(X)=\bigcup_{A \in \mathcal{T}(\Psi)}(X \ominus A) \cap\left[X^{c} \ominus(W \backslash A)\right]=\bigcup_{A \in \mathcal{T}(\Psi)} X \otimes(A, W \backslash A) .
$$

Thus $\Psi(X)$ is the the set of points $\mathbf{p}$ at which at least one of the shapes $A$ in the collection $\mathcal{T}(\Psi)$, shifted at location $\mathbf{p}$, fits exactly inside $X$.

Obviously, any window transformation $\Psi$ is TI. Hence, it can be represented by its kernel $\operatorname{Ker}(\Psi)=\{X: \mathbf{0} \in \Psi(X)\}=\{X: W \cap X \in \mathcal{T}(\Psi)\}$. If $W=\mathbb{Z}^{m}$, then $\mathcal{T}(\Psi)=\operatorname{Ker}(\Psi)$.

A discrete set operator $\Phi$ is called locally-defined if there exists a finite window $W \subseteq \mathbb{Z}^{m}$ such that

$$
\mathbf{p} \in \Phi(X) \Longleftrightarrow \mathbf{p} \in \Phi\left(X \cap W_{+\mathbf{p}}\right)
$$

for all $X \subseteq \mathbb{Z}^{m}$. Thus, the value of the output $\Phi(X)$ at each point depends only on the values of the input $X$ within the window $W$ shifted at that point. There is a very close relationship between window transformations and locally-defined operators as the following result reveals.

Proposition 19. A set operator on $\mathcal{P}\left(\mathbb{Z}^{m}\right)$ is a window transformation if and only if it is a locally-defined TI operator.

Henceforth, we shall use interchangeably the equivalent concepts of a 'window operator' and a 'TI locally-defined operator'. Next we compare the representation of window operators based on their shape collection versus their basis.

Theorem 23 (Heijmans, 1987).

Let $\Phi: \mathcal{P}\left(\mathbb{Z}^{m}\right) \rightarrow \mathcal{P}\left(\mathbb{Z}^{m}\right)$ be an increasing TI locally-defined operator. Then

(a) $\Phi$ can be represented as a finite union of erosions; i.e.,

$$
\Phi(X)=\bigcup_{A \in \mathcal{T}(\Phi)} X \ominus A
$$

(b) $\Phi$ is upper semi-continuous.

So far we have seen that any increasing window operator, can be represented either as a finite union of hit-miss transformations or as a finite union of erosions. These representations are important because increasing finite-window transformations encompass all rank operators, stack filters, and increasing discrete morphological operators that use finite structuring elements. However, they are computationally less efficient than the representation of all these operators via erosions by the basis elements. The reason is that, for any increasing finite-window operator $\Psi$, both $\mathcal{T}(\Psi)$ and its basis $\operatorname{Bas}(\Psi)$ are finite but the basis contains fewer elements than $\mathcal{T}$. To see this, let $F \subseteq G \subseteq W$ with $F \neq G$ and $\mathbf{0} \in \Psi(F) \subseteq \Psi(G)$. Then both subsets $F$ and $G$ of $W$ belong to $\mathcal{T}(\Psi)$, but only $F$ may belong to $\operatorname{Bas}(\Psi)$ because $G$ is not a minimal kernel element. Thus, we have the general result

$$
\operatorname{Bas}(\Psi) \subseteq \mathcal{T}(\Psi) \subseteq \operatorname{Ker}(\Psi)
$$

Example 16 . Let $W$ be the 5-pixel symmetric rhombus and consider the set median $\Psi(X)=$ $\operatorname{med}_{W}(X)$. Then, $\Psi$ is a window operator whose shape collection is $\mathcal{T}=\{A \subseteq W: 3 \leq|A| \leq 5\}$. Thus, $\mathcal{T}$ consists of 16 subsets of $W$; these subsets are the shapes that the window transformation detects. The basis of $\Psi$ is $\mathcal{B}=\{A \subseteq W:|A|=3\}$. Thus, $\mathcal{B}$ contains only 10 sets, and Theorem 21 represents $\Psi$ as the union of 10 erosions by these basis sets. However, Theorem 22 represents $\Psi$ as the union of 16 hit-miss transformations by the elements of $\mathcal{T}$, and each hit-miss transformation is the intersection of two erosions; hence, the latter representation is computationally less efficient because it requires a larger number of more complex morphological operations. Similarly, Theorem 23a represents $\Psi$ as a union of 16 erosions, which is less efficient than the 10 erosions required by the basis representation. 
The previous analysis gives us a very useful result.

Proposition 20. If $\Psi: \mathcal{P}\left(\mathbb{Z}^{m}\right) \rightarrow \mathcal{P}\left(\mathbb{Z}^{m}\right)$ be a discrete set operator. If $\Psi$ is an increasing window operator, then its basis is finite.

The basis representation of window operators applies if they are increasing. The increasingness allows us to easily extend these operators to graylevel image processing by building flat operators that process all the level sets of the input graylevel image using a binary window operator. A lattice-theoretic study of more general binary window operators that may be non-increasing was done in Barrera and Salas (1996) where they were used as building blocks of computational morphological machines. Further, Barrera and Dougherty (1998) investigated an extension of binary window operators to graylevel image processing using weighted windows(i.e. windows with graylevel weights), but the computational complexity is larger.

Concluding this section on the basis representations of increasing TI set operators (including morphological, median and rank filters, and window-based feature/object detection operators), such representations may be useful for alternative parallel implementations (hardware or software) of the corresponding binary image processing systems, as explained in Maragos (1989a). Another application area is optimal restoration of binary images (e.g. documents) in the presence of noise by using a union of erosions by a small number sets from a subcollection of the basis (Loce and Dougherty, 1992b). They also apply to graylevel image processing in the case of flat operators defined based on a finite window, which, as explained next, also admit a basis representation as maximum of flat erosions by the minimal kernel sets of their set generators.

\subsection{Kernel and Basis Representations for TI Increasing Function Operators}

In Sections 5.1 and 5.2 we discussed a representation theory by which every set operator that is translation-invariant (TI) and increasing can be expressed as a union of erosions by its kernel sets or as an intersection of dilations. If additionally the operator is upper semi-continuous (u.s.c.), then this union of erosions needs only the minimal kernel elements, i.e. the sets of its basis.

In this section we summarize a similar representation theory that has been developed for signal and graylevel image operators and provide some examples, e.g. its application to some nonlinear filters (openings, median and stack filters) and some linear filters (digital FIR filters).

\subsubsection{Representation of Weighted Operators and Basis Approximations}

For every TI, increasing, and u.s.c. signal operator there is a special collection of functions, called its basis, such that the operator can be represented as a supremum of morphological erosions by its basis functions. As for the case of TI set operators, this basis is a subcollection of a suitably defined kernel. Specifically, let $\psi$ be a signal operator on $\operatorname{Fun}\left(\mathbb{E}^{m}, \overline{\mathbb{R}}\right)$, i.e., the set of extended-realvalued functions defined on $\mathbb{E}^{m}=\mathbb{R}^{m}$ or $\mathbb{Z}^{m}$, and let $\psi^{*}(f)=-\psi(-f)$ be its dual (a.k.a. negative) operator. Let

$$
\operatorname{Ker}(\psi) \triangleq\{f: \psi(f)(\mathbf{0}) \geq 0\}
$$

be the kernel of $\psi$. This collection of signals can uniquely represent the operator, as the following result reveals.

Theorem 24 (Maragos, 1985, 1989a).

If $\psi$ is a TI and increasing operator on $\operatorname{Fun}\left(\mathbb{E}^{m}, \overline{\mathbb{R}}\right)$, then it can be represented as supremum of weighted erosions by the functions of its kernel and as infimum of weighted dilations by the reflected functions of the kernel of its dual operator:

$$
\psi(f)=\bigvee_{g \in \operatorname{Ker}(\psi)} f \ominus g=\bigwedge_{h \in \operatorname{Ker}\left(\psi^{*}\right)} f \oplus h^{s}
$$


In the above theorem $h^{s}(\mathbf{x})=h(-\mathbf{x})$ denotes the reflection of a function, and the function dilations and erosions are of the weighted type, defined in (60) and (61).

We can improve this representation by using fewer erosions as follows. The basis $\operatorname{Bas}(\psi)$ is defined as the collection of the minimal (w.r.t. $\leq$ ) kernel functions:

$$
\operatorname{Bas}(\psi) \triangleq\{g \in \operatorname{Ker}(\psi):[f \in \operatorname{Ker}(\psi) \text { and } f \leq g] \Longrightarrow f=g\}
$$

If we limit to u.s.c. operators acting on the class of u.s.c. functions, then the basis exists and can fully represent the operator, as explained next.

Theorem 25 (Maragos, 1985, 1989a).

(a) If $\psi$ is a TI, and increasing and u.s.c. operator on $\mathrm{Fun}_{u s c}\left(\mathbb{E}^{m}, \overline{\mathbb{R}}\right)$, then it can be represented as supremum of weighted erosions by the functions of its basis.

(b) If $\mathbb{E}^{m}=\mathbb{Z}^{m}$ and the dual operator is also u.s.c., the $\psi$ can also be represented as the infimum of weighted dilations by the reflected functions of the basis of its dual operator:

$$
\psi(f)=\bigvee_{g \in \operatorname{Bas}(\psi)} f \ominus g=\bigwedge_{h \in \operatorname{Bas}\left(\psi^{*}\right)} f \oplus h^{s}
$$

Thus, the above theorem represents exactly any TI increasing and u.s.c. operator by using a full expansion of erosions by all its basis functions (and dually as a dilation expansion). What happens if we use only a subcollection of the basis functions in the above representation? Such a question arises often in practical image processing applications such as denoising where an optimum system needs to be designed based on a finite small number of erosions (Loce and Dougherty, 1992b, 1995). The following result is a straightforward consequence of Theorem 25(b).

Proposition 21 (Approximate Basis Representation):

If in the basis representation (216) we use smaller collections $\mathcal{B} \subset \operatorname{Bas}(\psi)$ and $\mathcal{B}^{*} \subset \operatorname{Bas}\left(\psi^{*}\right)$ than the bases of the operators $\psi$ and $\psi^{*}$, respectively, of Theorem 25(b), and we create the operators

$$
\psi_{\ell}(f) \triangleq \bigvee_{g \in \mathcal{B}} f \ominus g, \quad \psi_{u}(f) \triangleq \bigwedge_{h \in \mathcal{B}^{*}} f \oplus h^{s}
$$

then the original operator $\psi$ is bounded from below and above by these two operators with the truncated bases:

$$
\psi_{\ell}(f) \leq \psi(f) \leq \psi_{u}(f), \quad \forall f .
$$

For cases where all the basis functions are finite-valued on the same subset of the general domain (e.g., such a case is the basis of increasing linear translation-invariant filters discussed in Section 5.4.4), Dougherty and Kraus (1991) have found a tight error bound in the approximation of an operator when removing one basis functions from the full erosion expansion.

The bounding result (217) assumed that we already had a TI increasing operator whose basis was truncated to create a new approximate operator. Another direction is to synthesize a collection of functions possessing the fundamental property of a morphological basis, i.e. its elements must be minimal, and then construct an operator as supremum of erosions by these basis functions:

\section{Proposition 22 .}

(a) Let $\mathcal{B}$ be a collection of functions such that all elements of $\mathcal{B}$ are minimal in $(\mathcal{B}, \leq)$ and define the operator

$$
\psi(f) \triangleq \bigvee_{g \in \mathcal{B}} f \ominus g
$$

Then $\psi$ is a TI and increasing operator whose basis is equal to $\mathcal{B}$.

(b) Let $\mathcal{B}^{*}$ be a collection of functions such that all elements of $\mathcal{B}^{*}$ are minimal in $\left(\mathcal{B}^{*}, \leq\right)$ and define the operator

$$
\phi(f) \triangleq \bigwedge_{h \in \mathcal{B}^{*}} f \oplus h^{s}
$$

Then $\phi$ is a TI and increasing operator whose dual operator $\phi^{*}$ has $\mathcal{B}^{*}$ as its basis. 
Thus, the morphological basis plays a conceptually similar role as a Hamel basis in a linear space. The minimality condition between two distinct functions $g_{1}$ and $g_{2}$ in a morphological basis implies that there exist points $\mathbf{x}$ and $\mathbf{y}$ such that

$$
g_{1}(\mathbf{x})>g_{2}(\mathbf{x}) \text { and } g_{1}(\mathbf{y})<g_{2}(\mathbf{y})
$$

In other words, inside the morphological basis we cannot find two distinct elements such that one contains (w.r.t. the partial order) or is contained by the other. All the elements in a basis $\mathcal{B}$ are atoms in the poset $(\mathcal{B}, \leq)$. Thus, the elements of a morphological basis are 'independent' in the sense of being minimal and can synthesize an operator via supremum. Next, we proceed with the example of a graylevel image operator that possesses a finite basis. In Section 5.4.4 we shall present an application of the basis representation Theorem 25 to linear filters too.

Example 17 (Basis of Weighted Opening):

Consider the TI weighted opening of discrete-domain input signals $f \in \operatorname{Fun}\left(\mathbb{Z}^{m}, \overline{\mathbb{R}}\right)$ by a non-flat (structuring function) kernel $k(\mathbf{x})$ :

$$
(f \circ k)(\mathbf{x})=[(f \ominus k) \oplus k](\mathbf{x})=\bigvee_{\mathbf{z}} \bigwedge_{\mathbf{y}} f(\mathbf{x}+\mathbf{y}-\mathbf{z})-k(\mathbf{y})+k(\mathbf{z})
$$

From Proposition 22 it follows that this operator has a basis that consists of the functions in the following collection

$$
\operatorname{Bas}(f \mapsto f \circ k)=\left\{g: g(\mathbf{x})=k(\mathbf{x}+\mathbf{z})-k(\mathbf{z}), \mathbf{z} \in \operatorname{Spt}_{\mathbf{V}}(k)\right\}
$$

where $\operatorname{Spt}_{\vee}(k)=\{\mathbf{x}: k(\mathbf{x})>-\infty\}$ is the support of $k(\mathbf{x})$. Assuming, as usually done in imaging applications, that $k$ has a finite support yields a finite basis. Note, however, that the above results also hold for structuring functions $k(\mathbf{x})$ with infinite support and for continuous-domain openings.

Morales and Acharya (1993) have analyzed the above discrete opening for 1D signals and found its finite basis. Then, this was used to efficiently implement the 1D discrete opening and closing by $k$ using a block matrix method in Ko et al. (1995).

\subsubsection{Representation of Flat Operators}

Consider now a signal operator $\phi$ on $\operatorname{Fun}\left(\mathbb{E}^{m}, \overline{\mathbb{R}}\right)$ that is flat, i.e., for binary inputs it yields binary outputs. Let $\Phi$ be its corresponding set operator; i.e. $\Phi$ explains the action of $\phi$ on binary signals represented by sets. If $\phi$ commutes with thresholding, i.e.

$$
X_{v}[\phi(f)]=\Phi\left[X_{v}(f)\right], \quad v \in \mathbb{R}
$$

where $X_{v}(f)=\left\{\mathbf{x} \in \mathbb{E}^{m}: f(\mathbf{x}) \geq v\right\}$ are the level sets of $f$, then $\phi$ can be constructed by its set operator $\Phi$ via threshold superposition (Maragos and Ziff, 1990):

$$
\phi(\mathbf{x})=\sup \left\{v: \mathbf{x} \in \Phi\left[X_{v}(f)\right]\right\}
$$

Thus, $\Phi$ is called the set generator of $\phi$. The flat operator $\phi$ is TI and increasing if and only if its set generator $\Phi$ is TI and increasing. Further, the commuting with thresholding makes both $\phi$ and $\Phi$ increasing and u.s.c. Then, Theorem 21 and (224) give us the following simpler representation for flat operators involving flat erosions and dilations, defined in (58) and (59).

Theorem 26 (Maragos, 1985, 1989a).

(a) If $\phi$ is a TI flat operator on $\mathrm{Fun}_{\text {usc }}\left(\mathbb{E}^{m}, \overline{\mathbb{R}}\right)$ that commutes with thresholding and $\Phi$ is its set generator, then $\phi$ can be represented as the supremum of flat erosions by the basis sets of its set generator $\Phi$.

(b) Under the assumptions of (a), if we have a discrete signal domain $\mathbb{E}^{m}=\mathbb{Z}^{m}$ and the dual set generator $\Phi^{*}$ is u.s.c., then $\phi$ can also be represented as the infimum of flat dilations by the reflected dual basis sets:

$$
\phi(f)=\bigvee_{M \in \operatorname{Bas}(\Phi)} f \ominus M=\bigvee_{N \in \operatorname{Bas}\left(\Phi^{*}\right)} f \oplus N^{s}
$$


The above morphological basis representations (both for flat and weighted operators) have been applied to various classes of image operators, including morphological, median, stack, and linear filters (Maragos and Schafer, 1987a,b). One of their most attractive aspects is the capability of parallel implementations of a large class of systems, in a single pass, using simple local operations that do not involve multiplications. These representations of TI increasing operators as supremum of erosions (sup-inf operators) or as infimum of dilations (inf-sup operators) have proven to be very useful in (Catte et al., 1995) for nonlinear scale-space analysis and image enhancement using anisotropic diffusion and for solving a large variety of similar problems in image processing using PDEs (Guichard and Morel, 2001; Guichard et al., 2005). A versatile class of sup-inf operators was synthesized in Catte et al. (1995) as supremum of flat erosions by equal-length symmetric straight line segments oriented at all possible angles; this collection of line segments is a basis, as explained by Proposition 22. More recently, they have also been used in (Alvarez et al., 2010) to simplify the discretization of the PDEs driving geodesic active contours and their implementation via level sets for solving computer vision problems.

We provide below an application of the flat operator representation Theorem 26 to median filtering of graylevel images and in the next section we explain how it also applies to a broad class of nonlinear digital filters, called stack filters, which contain the median as special case.

Example 18 (2D Flat Median):

Consider digital images on $\mathbb{Z}^{2}$, let $W=\{(0,0),(0,1),(1,0),(-1,0),(0,-1)\}$ be the rhombus-shaped 5-point discrete disk of unit radius, let $\phi(f)=\operatorname{med}_{W}(f)$ be the graylevel median filter w.r.t. window $W$, and let $\Phi(X)=\operatorname{med}_{W}(X)$ be its corresponding set generator. The basis of $\Phi$ consists exactly of the ten 3-pixel subsets of $W$ which are shown in Fig. 9. By (206), the set generator can be represented as

$$
\operatorname{med}_{W}(X)=\bigcup_{A \subseteq W,|A|=3} X \ominus A=\bigcap_{B \subseteq W,|B|=3} X \oplus B^{s}
$$

Then, by Theorem 26, the graylevel median is expressed as a maximum of ten 3-point moving local minima:

$$
\operatorname{med}_{W}(f)(\mathbf{x})=\bigvee_{A \subseteq W,|A|=3} \min _{\mathbf{y} \in A}\{f(\mathbf{x}+\mathbf{y})\}=\bigwedge_{B \subseteq W,|B|=3} \max _{\mathbf{y} \in B}\{f(\mathbf{x}+\mathbf{y})\}
$$

This representation (Maragos and Schafer, 1987b) connects the morphological image analysis area with the order-statistics nonlinear filtering area, since any median and rank filter for graylevel images and real-valued signals can be expressed via a closed formula involving only a maximum (resp. minimum) of flat erosions (resp. dilations) by pre-specified subsets of the window without requiring any sorting inside the moving window. Beyond the possible implementational benefits (e.g. parallelism, local operations on pre-specified subwindows), a big scientific gain is the ability to analyze and design rank-based nonlinear filters using geometric operations (erosions and dilations) and mathematical logic, since the basis representation of the set generator is essentially a minimal representation of a Boolean function, as explained in the next section.

\subsubsection{Representations of Boolean Functions and Stack Filters}

Boolean Functions The representation theory of discrete set operators as union of erosions or as intersection of dilations has direct analogies with the representation of Boolean functions. Before we can make this precise, we need a few definitions from the theory of Boolean functions (Kohavi, 1978; Muroga, 1971). Any Boolean expression of $n$ variables $v_{1}, v_{2}, \ldots, v_{n} \in\{0,1\}$ can be written as Boolean sum-of-products (SOP) terms or as Boolean product-of-sum (POS) terms. Each product or sum term may contain each literal (a variable or its complement) at most once and/or the Boolean constants 0 or 1 . To each Boolean expression there corresponds a unique Boolean function $b(\mathbf{v}) \in\{0,1\}$, where $\mathbf{v}=\left(v_{1}, v_{2}, \ldots, v_{n}\right)$. A Boolean function is usually described through a truth table. Two Boolean expressions are called equivalent if they correspond to the same Boolean function. A Boolean function $b_{1}$ is said to imply $b_{2}$ iff $b_{2}(\mathbf{v})=1$ for each $\mathbf{v}$ such that $b_{1}(\mathbf{v})=1$. A 
prime implicant $\pi$ of $b$ is a product term which implies $b$, such that deletion of any literal from $\pi$ results in a new product which does not imply $b$. A prime implicate of $b$ is a sum term $\sigma$ implied by $b$, such that deletion of any literal from $\sigma$ results in a new sum term which is not implied by $b$. Any minimal SOP (resp. POS) expression for $b$ is a sum (resp. product) of prime implicants (resp. prime implicates) such that removal of any of them makes the remaining expression no longer equivalent to $b$, and the expression contains the minimum number of literals and product (resp. sum) terms. This minimal expression is not necessarily unique. A function $b(\mathbf{v})$ is called positive if it can be represented by a SOP or POS expression in which no variable appears in uncomplemented form. Each positive function has a unique minimal SOP expression that is positive and is the sum of all its prime implicants; it also has a unique minimal POS expression that is positive and is the product of all its prime implicates.

Consider now from Example 2 the lattice $B V_{n}=\{0,1\}^{n}$ of $n$-variable Boolean vectors and the lattice $B F_{n}$ of Boolean functions defined on $B V_{n}$. A Boolean function $b$ is called increasing if $\mathbf{v} \leq \mathbf{u}$ implies that $b(\mathbf{v}) \leq b(\mathbf{u})$. A Boolean function is increasing if and only if it is positive (Gilbert, 1954). If $b$ is positive, its dual function $b^{*}(\mathbf{v})=\overline{b(\overline{\mathbf{v}})}$ is positive too, where $\overline{(\cdot)}$ denotes Boolean complement.

In $B V_{n}$, a vector $\mathbf{x}$ is called a minimal true vector of a Boolean function $b$ iff $b(\mathbf{x})=1$ and $\mathbf{x}$ is not preceded (with respect to the vector partial ordering) by any other vector $\mathbf{v}$ with $b(\mathbf{v})=1$. Dually, a vector $\mathbf{y}$ is called a maximal false vector of $b$ iff $b(\mathbf{y})=0$ and $\mathbf{y}$ is not followed by any other vector $\mathbf{u}$ with $b(\mathbf{u})=0$.

Boolean functions can generate TI digital signal and image operators. Consider an $m$-dimensional digital binary image signal $\mathbb{1}_{S}(\mathbf{x}): \mathbb{Z}^{m} \rightarrow\{0,1\}$ with values 1 for the image foreground $S \subseteq \mathbb{Z}^{m}$ and 0 for the background $S^{c}$. This is actually the set's binary indicator function:

$$
\mathbb{1}_{S}(\mathbf{x})= \begin{cases}1, & \mathbf{x} \in S \\ 0, & \mathbf{x} \in S^{c}\end{cases}
$$

Typical local image transformations involving a neighborhood of $n$ samples whose indices are arranged in a finite window set $W=\left\{\mathbf{p}_{1}, \mathbf{p}_{2}, \ldots, \mathbf{p}_{n}\right\} \subseteq \mathbb{Z}^{m}$ can be expressed as Boolean filtering of the binary signal $\mathbb{1}_{S}$ corresponding to the input image set $S$ :

$$
\phi_{b}\left(\mathbb{1}_{S}\right)(\mathbf{x}) \triangleq b\left(\mathbb{1}_{S}\left(\mathbf{x}+\mathbf{p}_{1}\right), \ldots, \mathbb{1}_{S}\left(\mathbf{x}+\mathbf{p}_{n}\right)\right)
$$

where $b\left(v_{1}, \ldots, v_{n}\right)$ is a Boolean function of $n$ variables The mapping $\mathbb{1}_{S} \mapsto \phi_{b}\left(\mathbb{1}_{S}\right)$ is a nonlinear shift-invariant operator for binary signals, called a Boolean filter or Boolean operator. This has a unique correspondence with a TI set operator $\Phi_{b}$ by replacing input and output binary signals with sets:

$$
\phi_{b}\left(\mathbb{1}_{S}\right)=\mathbb{1}_{\Phi_{b}(S)}
$$

The dual Boolean function $b^{*}$ will generate the dual set operator $\Phi_{b}{ }^{*}$. Assume henceforth that $b$ is increasing; then, both $\Phi_{b}$ and its dual are TI, increasing and u.s.c. discrete set operators on $\mathcal{P}\left(\mathbb{Z}^{m}\right)$. Hence, by Theorem $21, \Phi_{b}$ can be represented as the union of erosions by its basis sets and as the intersection of dilations by the reflected basis sets of its dual operator. Then we have a one-to-one correspondence between the basis sets of the set operator, which are subsets of $W$, and the minimal true vectors of the Boolean function (Maragos and Schafer, 1987b).

For example, if we analyze 1D binary signals on $\mathbb{Z}$ with Boolean operations inside a moving 3-point window $W=\{-1,0,1\}$, consider the Boolean filter generated by the Boolean function (written as a SOP and a POS expression)

$$
b\left(v_{1}, v_{2}, v_{3}\right)=v_{1} v_{2}+v_{2} v_{3}=v_{2}\left(v_{1}+v_{3}\right)
$$

where the indexing of the Boolean variables $v_{i}$ is $i=w+2, w \in W$. The binary filter follows from the general definition (229):

$$
\phi_{b}\left(\mathbb{1}_{S}\right)(x)=b\left(\mathbb{1}_{S}(x-1), \mathbb{1}_{S}(x), \mathbb{1}_{S}(x+1)\right), \quad x \in \mathbb{Z}
$$


Each shift $\mathbb{1}_{S}(x+w), w \in W$, of the binary signal corresponds to a shift $S_{-w}$ of the input set. Thus, the set operator generated by the above Boolean function is

$$
\Phi_{b}(S)=\left(S_{+1} \cap S\right) \cup\left(S \cap S_{-1}\right)=S \cap\left(S_{-1} \cup S_{+1}\right), \quad S \subseteq \mathbb{Z}
$$

The basis of $\Phi_{b}$ is $\mathcal{B}=\{\{-1,0\},\{0,1\}\}$, and the basis of $\Phi_{b}{ }^{*}$ is $\mathcal{B}^{*}=\{\{0\},\{-1,1\}\}$. The minimal true vectors of $b$ are $(1,1,0)$ and $(0,1,1)$. Indeed, we confirm the one-to-one correspondence between the basis sets of $\Phi_{b}$ and the minimal true vectors of $b$. Dually, the maximal false vectors of $b$ are $(0,1,0)$ and $(1,0,1)$ which correspond to the basis sets of $\Phi_{b}{ }^{*}$.

Stack Filters Consider the class of digital positive real-valued signals $f$ with domain $\mathbb{Z}^{m}$ and quantized range $\operatorname{Ran}(f) \subseteq\{0,1,2, \ldots, M\}$. For such signals Wendt et al. (1986) defined the class of stack filters by using a positive Boolean function $b\left(v_{1}, \ldots, v_{n}\right)$ of $n$ variables (corresponding to positions in a moving window $W$ of $n$ points) to filter all binary signals corresponding to the level sets

$$
X_{t}(f)=\{\mathbf{x}: f(\mathbf{x}) \geq t\}
$$

and synthesizing the graylevel output signal via threshold superposition:

$$
\mathrm{ST}_{b}(f)(\mathbf{x}) \triangleq \sum_{t=1}^{M} \phi_{b}\left(\mathbb{1}_{X_{t}(f)}\right)(\mathbf{x})
$$

If we replace the pointwise summation in the above definition with supremum we can remove both the constraint of dealing with positive signals and the constraint of a quantized range (Maragos and Schafer, 1987b). For the cases when $b$ is a threshold function (Muroga, 1971), Wendt et al. (1986) provided a functional definition for $\mathrm{ST}_{b}$ as a generalization of rank filters, in which multiple repetitions of the same element are allowed. Further, Wendt et al. (1986) studied several of the deterministic and statistical properties of stack filters and analyzed in detail all stack filters corresponding to the 20 positive Boolean functions with $n=3$.

As we did for increasing Boolean functions, it is possible to use a basis representation for stack filters too. Details can be found in Maragos and Schafer (1987b). Here we outline the main ideas and illustrate them with an example. All stack filters are TI, increasing and u.s.c. operators on the class of real-valued discrete-domain signals. They are generated by a positive Boolean function $b$ or equivalently by a TI, increasing and u.s.c. set operator $\Phi_{b}$. Further, they are flat operators, i.e. yield binary outputs for binary inputs, and they commute with thresholding. The basis of their set generator $\Phi_{b}$ is finite and consists of all subsets of $W$ that correspond with the minimal true vectors of $b$. Thus, Theorem 26 can represent all stack filters exactly as a maximum of moving local minima inside the basis sets or as a minimum of moving local maxima inside the basis sets of the dual operator. For an example, if we use the 3-variable Boolean function $b$ of (231) as generator, the resulting stack filter is

$$
\begin{aligned}
\mathrm{ST}_{b}(f)(x) & =\max \{\min [f(x-1), f(x)], \min [f(x), f(x+1)]\} \\
& =\min \{f(x), \max [f(x-1), f(x+1)]\}
\end{aligned}
$$

We can observe that, if we have already expressed a positive Boolean function in its irreducible SOP or POS form, then the max-min representation of the corresponding stack filter follows directly by replacing Boolean OR with max and Boolean AND with min.

\subsubsection{Representation of Linear Operators via Morphological Operations}

Let $\Gamma$ be a linear shift-invariant (LSI) filter defined on a class of discrete-domain $\left(\mathbb{Z}^{m}\right)$ real-valued signals closed under translation, and let $h$ be its impulse response. Then its output can be found via linear convolution of the input signal with the impulse response:

$$
\Gamma(f)(\mathbf{x})=(h * f)(\mathbf{x})=\sum_{\mathbf{y} \in \mathbb{Z}^{m}} f(\mathbf{y}) h(\mathbf{x}-\mathbf{y}), \quad \mathbf{x} \in \mathbb{Z}^{m}
$$


Due to the linearity of $\Gamma$, it is identical with is dual (w.r.t. negation) operator: $\Gamma^{*}(f)=-\Gamma(-f)=$ $\Gamma(f)$. The LSI operator $\Gamma$ is increasing if and only if

$$
h(\mathbf{x}) \geq 0 \forall \mathbf{x},
$$

and translation-invariant if and only if

$$
\sum_{\mathbf{x}} h(\mathbf{x})=1
$$

Under the two above conditions we can represent the linear filter via morphological operations, as the following result explains. We restrict our discussion to discrete linear filters with finite impulse response (FIR), which covers most of the filters used in linear digital signal processing. The FIR constraint is a sufficient condition for the existence of a morphological basis.

Theorem 27 (Maragos and Schafer, 1987a).

Let $h(\mathbf{x}), \mathbf{x} \in \mathbb{Z}^{m}$, be the finite-extent impulse response of an $m$-dimensional linear shift-invariant filter $\Gamma(f)=f * h$, which is defined on a class $\mathcal{S}$ of real-valued discrete-domain signals $f: \mathbb{Z}^{m} \rightarrow \mathbb{R}$ closed under translation. If h satisfies the conditions (238), then the morphological basis of $\Gamma$ exists and is equal to

$$
\operatorname{Bas}(\Gamma)=\left\{g \in \mathcal{S}: \sum_{\mathbf{y} \in \operatorname{Spt}(h)} h(\mathbf{y}) g(-\mathbf{y})=0 \text { and } g(-\mathbf{x})=-\infty \Longleftrightarrow h(\mathbf{x})=0\right\}
$$

Further, the linear operator can be represented as a supremum of weighted erosions by its basis functions:

$$
\Gamma(f)(\mathbf{x})=(h * f)(\mathbf{x})=\bigvee_{g \in \operatorname{Bas}(\Gamma)} \bigwedge_{\mathbf{y} \in \mathbb{Z}^{m}} f(\mathbf{y})-g(\mathbf{y}-\mathbf{x})
$$

It can also be represented as an infimum of weighted dilations by the reflected functions of its basis.

A linear shift-invariant filter that satisfies the additional two conditions (238) is an increasing linear translation-invariant (ILTI) operator. Note that the sup-inf representation of such an operator in (240) requires two different ways of interpreting and representing the support of an input signal $f$. By 'support' we mean the set of samples that carry information affecting the operator. For linear convolutions with an impulse response $h$, the support of $h$ is the set of non-zero samples:

$$
\operatorname{Spt}(h) \triangleq\{\mathbf{x}: h(\mathbf{x}) \neq 0\}
$$

For morphological-type convolutions with a structuring function $g$ the role of the zeros in linear operations is now played by the $-\infty$ values; hence, the upper support is defined as follows:

$$
\operatorname{Spt}_{\vee}(g) \triangleq\{\mathbf{x}: g(\mathbf{x})>-\infty\}
$$

Now, for the double (linear and morphological) representation in (240), the 'support' of an input signal $f$ must be represented in two different ways. When $f$ is linearly convolved with $h$ in (240), we assume that $f(\mathbf{x})=0$ outside the support of $f$. However, when $f$ is morphologically convolved with $g$, we assume that $f(\mathbf{x})=-\infty$ outside the support of $f$.

Theorem 27 requires some constraints on the impulse response of the LSI filter, i.e., nonnegativity and sum of values equal to one. These constraints can relaxed by expressing the impulse response as the difference between two positive signals and normalizing them to have a unity DC-gain (Maragos and Schafer, 1987a).

Since a supremum of erosions involves only additions and max-min comparisons, the morpohological representation of a linear filter has the attractive feature of implementing linear systems without using multiplications. However, we need an infinite number of erosions to precisely build the linear filter. Specifically, the basis functions of the FIR ILTI operator $\Gamma$ are given by (239). 
They all have support on the set $[\operatorname{Spt}(h)]^{s}$ and form a finite-dimensional space, i.e. $\mathbb{R}^{N-1}$, where $N=|\operatorname{Spt}(h)|$ is the number of points in the support of $h$. Thus, the basis consists of an infinite number of functions, fully described with $N-1$ real parameters. As proposed in Maragos and Schafer (1987a), if we can use only a finite number of the required erosions to realize an ILTI system by quantizing and bounding the range of input signals and quantify the approximation errors, these morphological representations of linear systems may become practically useful. Such issues were investigated by Dougherty and Kraus (1991) and Koshravi and Schafer (1994). Dougherty and Kraus (1991) studied the properties of the basis of digital moving average filters and found a tight bound for the approximation error in a truncated erosion expansion which occurs when we remove one or more basis functions. By using a finite quantized range for the signals, Koshravi and Schafer (1994) developed practical implementations of FIR digital linear filters that require only a finite number of max-min operations and additions and studied the quantization error effects; these implementations include a decomposed structure that reduced to linear the exponential dependency on the number of quantization levels and cardinality of the support.

We finish our discussion on the morphological representation of linear filters with a simple example.

Example 19 (2-point Moving Average Linear filter):

Consider a discrete linear filter $f \mapsto \Gamma(f)=f * h$ for $1 \mathrm{D}$ signals $f(n)$ with impulse response

$$
h(n)=a \delta(n)+(1-a) \delta(n-1), \quad n \in \mathbb{Z},
$$

where $0<a<1$ and $\delta(n)$ is the discrete unit impulse. Thus, $\Gamma$ is a moving average filter on a 2-point moving window. Its basis elements are functions $g(n), n \in \mathbb{Z}$, defined by

$$
g(n)= \begin{cases}r \in \mathbb{R}, & n=0 \\ -a r /(1-a), & n=-1 \\ -\infty, & n \neq 0,-1\end{cases}
$$

Then, Theorem 27 yields the following representation for the linear filter

$$
(f * h)(n)=a f(n)+(1-a) f(n-1)=\sup _{r \in \mathbb{R}}\left[\min \left\{f(n)-r, f(n-1)+\frac{a r}{1-a}\right\}\right]
$$

which implements the linear convolution using only max-min operations and additions.

\subsection{Representations for Spatially-Varying Increasing Operators}

The most general representation theorem for increasing operators on complete lattices is the following.

Theorem 28 (Serra, 1988).

Let $\psi$ be an operator on a complete lattice $\mathcal{L}$. Then, $\psi$ is increasing with $\psi(I)=I$ if and only if $\psi$ is a supremum of a nonempty set of erosions:

$$
\psi(X)=\bigvee_{B \neq I} \varepsilon_{B}(X), \quad X \in \mathcal{L}
$$

where the erosions are defined by

$$
\varepsilon_{B}(X)= \begin{cases}I, & \text { if } \quad X=I \\ \psi(B), & \text { if } \quad I>X \geq B \\ O, & \text { if } \quad X \nsupseteq B\end{cases}
$$


Despite the generality of the above theorem, it is not practical because it employs too many erosions, i.e. as many as the number of lattice elements, and it is self-referencial since the erosions are defined in terms of the operator $\psi$ which they aim to synthesize.

Another approach to represent increasing image operators on the set lattice $\mathcal{P}\left(\mathbb{E}^{m}\right)$ and the function lattice $\operatorname{Fun}\left(\mathbb{E}^{m}, \overline{\mathbb{R}}\right)$ without the translation-invariance constraint has been developed by Bouaynaya et al. (2008) for binary images and by Bouaynaya and Schonfeld (2008) for graylevel images. This is based on spatially-varying $(S V)$ morphology and on extending the previous kernel and basis representation theorems for TI increasing operators to employ suprema and infima of SV erosions and dilations respectively. We shall summarize below the main ideas only for binary image (set) operators on $\mathcal{P}\left(\mathbb{E}^{m}\right)$.

Recall from Section 3.6 the concept of the structuring element map (SEM), proposed in (Serra, 1988, chap.2) where it was called a 'structuring function'. This is an SV structuring element, which in the case of sets is a map $\mathcal{A}(\mathbf{x}): \mathbb{E}^{m} \rightarrow \mathcal{P}\left(\mathbb{E}^{m}\right)$ that assigns a possibly different set $\mathcal{A}(\mathbf{x})$ at each point $\mathbf{x}$ of $\mathbb{E}^{m}$. Define its transposed SEM by

$$
\mathcal{A}^{s}(\mathbf{x}) \triangleq\left\{\mathbf{y} \in \mathbb{E}^{m}: \mathbf{x} \in \mathcal{A}(\mathbf{y})\right\}
$$

This helps us define an $S V$ adjunction $\left(\mathcal{E}_{\mathcal{A}}, \Delta_{\mathcal{A}}\right)$ of an adaptive dilation and erosion by $\mathcal{A}$ :

$$
\begin{aligned}
& \Delta_{\mathcal{A}}(X)=\left\{\mathbf{z} \in \mathbb{E}^{m}: \mathcal{A}^{s}(\mathbf{z}) \cap X \neq \varnothing\right\}=\bigcup_{\mathbf{x} \in X} \mathcal{A}(\mathbf{x}) \\
& \mathcal{E}_{\mathcal{A}}(X)=\left\{\mathbf{z} \in \mathbb{E}^{m}: \mathcal{A}(\mathbf{z}) \subseteq X\right\}=\bigcap_{\mathbf{y} \in X^{c}}\left(\mathcal{A}^{s}(\mathbf{y})\right)^{c}
\end{aligned}
$$

The two above adaptive operators are capable of representing any increasing operator; if the operator is also u.s.c., then the representation can become minimal. These representations need the concept of a kernel and a basis for adaptive operators. The main concepts are like the ones developed before in the translation-invariant case by Matheron (1975) and Maragos (1985), but extended to the SV case by Bouaynaya et al. (2008). Specifically, let $\psi$ be an SV operator on $\mathcal{P}\left(\mathbb{E}^{m}\right)$. Its SV kernel is defined as the following collection of SEMs

$$
\operatorname{Ker}_{s v}(\psi) \triangleq\left\{\mathcal{A}: \mathbf{x} \in \psi[\mathcal{A}(\mathbf{x})] \forall \mathbf{x} \in \mathbb{E}^{m}\right\}
$$

The subscript 'sv' in the kernel notation reminds us that it is for SV operators. The SV basis of $\psi$ is the collection $\operatorname{Bas}_{s v}(\psi)$ of minimal kernel elements.

Theorem 29 (Bouaynaya et al., 2008).

(a) An $S V$ operator $\psi$ on $\mathcal{P}\left(\mathbb{E}^{m}\right)$ is increasing if and only if it can be represented as the union of $S V$ erosions by the SEMs of its kernel or equivalently as the intersection of $S V$ dilations by the transposed SEMs of the kernel of its dual operator $\psi^{*}$.

(b) If $\psi$ is restricted on $\mathcal{F}\left(\mathbb{E}^{m}\right)$ and is u.s.c., then its basis exists and $\psi$ can be represented as the union of $S V$ erosions only by the SEMs of its basis. In addition, if $\psi^{*}$ is u.s.c, then $\psi$ can also be represented as the intersection of $S V$ dilations by the transposed SEMs of the basis of its dual:

$$
\psi(X)=\bigcup_{\mathcal{A} \in \operatorname{Bas}_{s v}(\psi)} \mathcal{E}_{\mathcal{A}}(X)=\bigcap_{\mathcal{A} \in \operatorname{Bas}_{s v}\left(\psi^{*}\right)} \mathcal{D}_{\mathcal{A}^{s}}(X)
$$

Thus, any increasing operator can be decomposed into a supremum of adaptive erosions (or infimum of adaptive dilations), and this decomposition can be minimal. These results unify the adaptive morphological operators based on SV neighborhoods with those based on group-invariant morphology. For example, the circular morphology (a.k.a. polar morphology) case by Roerdink and Heijmans (1988) and the affine morphology by Maragos (1990) were shown in Bouaynaya et al. (2008) to correspond to SV morphological operations with specific choices for an SEM. We illustrate Theorem 29 with two examples. 
Example 20 (Polar Morphology):

Consider set operators on $\mathcal{P}(E)$ where $E=\mathbb{R}^{2}-\{(0,0)\}$. For any points $\mathbf{x}=\left(r_{x}, \theta_{x}\right)$ and $\mathbf{y}=$ $\left(r_{y}, \theta_{y}\right)$ in $E$ expressed in polar coordinates, define the binary operation

$$
\mathbf{x} \odot \mathbf{y}=\left(r_{x} r_{y}, \theta_{x}+\theta_{y} \quad \bmod 2 \pi\right)
$$

which is multiplication on the complex plane. Based on this group operation, we can consider generalized translations

$$
\tau_{r, \theta}(X)=\{\mathbf{x} \odot(r, \theta): \mathbf{x} \in X\}
$$

that are rotations and scalar multiplications of planar sets. By combining these generalized translations for all vectors in a fixed nonempty planar set $B \subseteq E$, Roerdink and Heijmans (1988) defined the following two planar set operators that are invariant under rotations and scalar multiplications:

$$
\begin{aligned}
& X \oplus_{p} B \triangleq \bigcup_{(r, \theta) \in B} \tau_{r, \theta}(X) \\
& X \ominus_{p} B \triangleq \bigcap_{(r, \theta) \in B} \tau_{r, \theta}^{-1}(X)
\end{aligned}
$$

They are called polar dilation and polar erosion, respectively.

By using the above ideas, Bouaynaya et al. (2008) defined the following SEM

$$
\mathcal{A}(\mathbf{x})=B \odot \mathbf{x}=\{\mathbf{b} \odot \mathbf{x}: \mathbf{b} \in B\}
$$

which is the generalized translation of $B$ that rotates and scales the points in $B$ by the polar coordinates of $\mathbf{x}$. The transposed SEM is

$$
\mathcal{A}^{s}(\mathbf{x})=B^{-1} \odot \mathbf{x}
$$

where $B^{-1}=\left\{\left(r^{-1},-\theta\right):(r, \theta) \in B\right\}$. Thus, under the above formalism, the polar dilation (253) and polar erosion (254) become the SV dilation and erosion by the SEM of (255):

$$
\begin{aligned}
& X \oplus_{p} B=\left\{\mathbf{z} \in E:\left(B^{-1} \odot \mathbf{z}\right) \cap X \neq \varnothing\right\} \\
& X \ominus_{p} B=\{\mathbf{z} \in E: B \odot \mathbf{z} \subseteq X\}
\end{aligned}
$$

If we wish to represent these adaptive operators using the basis expansion (250), then their basis contains just one SEM.

Example 21 (Adaptive Binary Median filter):

Consider a SEM $\mathcal{W}: \mathbb{Z}^{m} \rightarrow \mathcal{P}\left(\mathbb{Z}^{m}\right)$ that works as an adaptive window for a $n$-point binary median filter $X \mapsto \operatorname{med}_{\mathcal{W}}(X)$. Namely, it moves to various positions $\mathbf{x}$ on a discrete domain as a spatiallyvarying set $\mathcal{W}(\mathbf{x})$ which may change its shape but its cardinality remains constant and equal to some odd integer $|\mathcal{W}(\mathbf{x})|=n$. For example, if $n=5$, the SV window may assume (according to some rule) at each location one out of three possible shapes: a 5-pixel horizontal line segment, or a 5 -pixel vertical line segment, or a 5 -pixel rhombus. This operator is increasing but spatially-varying. Its SV basis is equal to (Bouaynaya et al., 2008)

$$
\operatorname{Bas}_{s v}\left(\operatorname{med}_{\mathcal{W}}\right)=\{\mathcal{A}: \mathcal{A} \subseteq \mathcal{W} \text { and }|\mathcal{A}|=(n+1) / 2\}
$$

Thus, by Theorem 29, this adaptive median can be represented as the union of SV erosions by the SEMs of the above basis, whose cardinality is $\left(\begin{array}{c}n \\ r\end{array}\right)$ with $r=(n+1) / 2$. In the presence of non-stationary noise, the adaptive median can provide a more efficient denoising than a translationinvariant median with the same window size. 


\subsection{Representations for TI Non-Increasing Operators}

So far all the representation theorems in Section 5 (with the exception of Theorem 22) referred to the realization of translation-invariant (TI) increasing operators via erosions or dilations. However, it is possible to use kernels to represent TI operators that are not necessarily increasing via operations closely related to hit-miss transformations. Specifically, consider the complete lattice ${ }^{25} \mathcal{P}\left(\mathbb{E}^{m}\right)$, of subsets of the Euclidean space $\mathbb{E}^{m}=\mathbb{R}^{m}$ or its discrete version $\mathbb{Z}^{m}$, and define a closed interval $[A, B] \subseteq \mathcal{P}\left(\mathbb{E}^{m}\right)$ by

$$
[A, B] \triangleq\left\{X \subseteq \mathbb{E}^{m}: A \subseteq X \subseteq B\right\}, \quad A, B \subseteq \mathbb{E}^{m} .
$$

The sets $A$ and $B$ are called the extremities of this interval.

The building blocks of general TI operators will be the following two operators that are closely

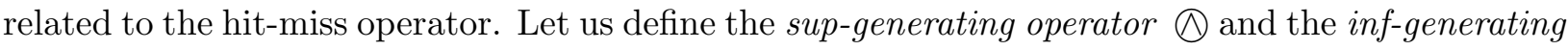
operator $\otimes$ by

$$
\begin{aligned}
X \otimes(A, B) & \triangleq\left\{\mathbf{x} \in \mathbb{E}^{m}: A_{+\mathbf{x}} \subseteq X \subseteq B_{+\mathbf{x}}\right\} \\
& =(X \ominus A) \cap\left(X^{c} \ominus B^{c}\right) \\
X \oslash(A, B) & \triangleq\left\{\mathbf{x}:\left(A^{s}\right)_{+\mathbf{x}} \cap X \neq \varnothing \text { or }\left(B^{s}\right)_{+\mathbf{x}} \cap X \neq \mathbb{E}^{m}\right\} \\
& =(X \oplus A) \cup\left(X^{c} \oplus B^{c}\right)
\end{aligned}
$$

The sets $(A, B)$ play the role of parameters for these operators. The names of these two operators above are due to the fact that, as explained next, they can generate arbitrary TI set operators by forming a union (supremum) or intersection (infimum) of such mappings selected from a class that specifies the operator. Recall the definition of the hit-miss operator

$$
X \otimes(A, B)=\left\{\mathbf{x} \in \mathbb{E}^{m}: A_{+\mathbf{x}} \subseteq X \text { and } B_{+\mathbf{x}} \subseteq X^{c}\right\}
$$

where $A$ is the (positive) template hitting the foreground of the binary image $X$, whereas $B$ is the (negative) template hitting the background. Note that the sup-generating operator is essentially the hit-miss operator with the exception of a complementation on the negative template:

$$
X \otimes(A, B)=X \otimes\left(A, B^{c}\right)
$$

Further, the inf-generating operator is also closely related by duality:

$$
X \otimes(A, B)=\left[X^{c} \otimes\left(A^{s}, B^{s}\right)\right]^{c}
$$

Now, as the following theorem explains, any TI set operator can be expressed as combination of these sup/inf-generating operators by set pairs related to closed intervals inside the kernel.

Theorem 30 (Banon and Barrera, 1991).

Any TI set operator $\psi: \mathcal{P}\left(\mathbb{E}^{m}\right) \rightarrow \mathcal{P}\left(\mathbb{E}^{m}\right)$ can be represented as the union of sup-generating operators by pairs of sets that form closed intervals in its kernel:

$$
\psi(X)=\bigcup_{[A, B] \subseteq \operatorname{Ker}(\psi)} X \otimes(A, B)
$$

Further, $\psi$ can be represented as the intersection of inf-generating operators by pairs of reflected sets that form intervals in the kernel of its dual operator:

$$
\psi(X)=\bigcap_{[A, B] \subseteq \operatorname{Ker}\left(\psi^{*}\right)} X \otimes\left(A^{s}, B^{s}\right)
$$

\footnotetext{
${ }^{25}$ Actually, the representation theory developed by Banon and Barrera (1991) for TI set operators assumes as image domain an arbitrary nonempty set $E$ equipped with a commutative group structure whose binary operation is denoted by + , and the lattice of image sets is $\mathcal{P}(E)$. In this case, 'translation-invariance' means invariance w.r.t. generalized translations formed by this group operation.
} 
The essence of the above theorem is that, any TI set operator can be expressed as union or intersection of hit-miss operations by set pairs that are extremities of closed intervals in the kernel. Barrera and Salas (1996) have further studied this representation as applied to finite window operators, by developing an algebra of lattice operations on closed intervals of sets, and applied their results to the automatic programming of morphological machines.

Banon \& Barrera's representation using hit-miss operations by kernel set intervals generalizes Matheron's representation using erosions or dilations by individual kernel sets. Of course, if we add the extra assumption that the TI set operator is also increasing, then Theorem 30 reduces to Matheron's Theorem 19. However, instead of adding the 'strong' assumption that $\psi$ is increasing, we can alternatively add one of the following two weaker assumptions. A set operator $\psi$ is called:

$$
\begin{aligned}
\text { inf-separable: } & X \subseteq Z \subseteq Y \Longrightarrow \psi(X) \cap \psi(Y) \subseteq \psi(Z) \\
\text { sup-separable: } & X \subseteq Z \subseteq Y \Longrightarrow \psi(Z) \subseteq \psi(X) \cup \psi(Y)
\end{aligned}
$$

Note that, any increasing and decreasing operators are both inf-separable and sup-separable. The following result is a corollary of Theorem 30 .

Proposition 23 (Banon and Barrera, 1991).

Let $\psi$ be a TI set operator on $\mathcal{P}\left(\mathbb{E}^{m}\right)$.

(a) If $\psi$ is inf-separable, then

$$
\psi(X)=\left(\bigcup_{A \in \operatorname{Ker}(\psi)} X \ominus A\right) \cap\left(\bigcup_{B \in \operatorname{Ker}(\psi)} X^{c} \ominus B^{c}\right)
$$

(b) If $\psi$ is sup-separable, then

$$
\psi(X)=\left(\bigcap_{A \in \operatorname{Ker}\left(\psi^{*}\right)} X \oplus A^{s}\right) \cup\left(\bigcap_{B \in \operatorname{Ker}\left(\psi^{*}\right)} X^{c} \oplus\left(B^{s}\right)^{c}\right)
$$

The name 'inf-separable' reflects the fact such mappings are an intersection (infimum) of two parts: an increasing part which is a union of erosions and a decreasing part which is a union of anti-dilations. Another simplification we obtain in the inf-separable case is that the required erosions and anti-dilations are by individual kernel sets without the need for operations with set intervals. Similarly, the name 'sup-separable' means that such mappings are a union (supremum) of two parts: an increasing part which is an intersection of dilations and a decreasing part which is an intersection of anti-erosions. If a TI inf-separable operator becomes increasing, then the decreasing part in its representation (268) vanishes and we are left only with its increasing part which is the regular union of erosions by kernel sets. Similarly, the decreasing part of a sup-separable operator disappears if the operator is increasing and leaves us only with the intersection of dilations by reflected dual kernel sets.

For a minimal representation, Banon and Barrera (1991) have developed a different concept of a basis of the kernel of TI operators, suitable for their sup-generating and inf-generating operators. They defined this new basis as the collection of maximal closed intervals of the kernel. To guarantee existence of this new basis they had to add the usual topological structure to the space $\mathcal{P}\left(\mathbb{E}^{m}\right)$, i.e. restrict to closed sets and use the hit-miss topology. Then, by using an approach similar to that used by Maragos (1985), they showed each TI and u.s.c set operator possesses a basis and can be represented as a union of sup-generating operations by pairs of sets that form intervals only inside the basis.

Let us compare the above minimal representation with the standard basis representation of Theorem 21 for increasing TI operators. Since every hit-miss operation detects the locations of a template $(A, B)$, which has a positive part $(A)$ hitting the foreground and a negative part $(B)$ hitting the background, we see that the above representation acts like a Boolean function that 
can be expressed as a Boolean sum of products (SOP) or as a Boolean product of sums (POS). Considering only the expansion with sup-generating operations, i.e. the SOP expression, we see that each Boolean product corresponds to a single hit-miss operation where the uncomplemented variables correspond to points of $A$ whereas the complemented variables correspond to points of $B$. If we compare this Boolean interpretation of the representation with hit-miss operations against the corresponding Boolean interpretation of increasing TI binary filters, which was explained in Section 5.4.3, we observe that in the latter case the Boolean function is irreducible and has only positive terms (i.e. no complementations). Thus, as also illustrated in Example 16, for an increasing TI set operator, if we use as basis elements the maximal intervals in the kernel, then these intervals may overlap which may create redundancies in the corresponding representation as union of hitmiss operations. In contrast, the basis using the minimal kernel elements has no redundancies and synthesizes the operator as union of simpler operations (erosions).

We conclude this section by mentioning that, many of the above concepts and representation results have been extended by Banon and Barrera (1993) to general complete lattices.

\subsection{Representations for TI Increasing Operators on Complete Weighted Lat- tices}

In this section we extend the kernel representation theory for increasing $\mathbb{T}$-invariant operators $\Psi$ on complete weighted lattice $(\mathrm{CWL})$ signal spaces $\mathcal{S}=\operatorname{Fun}\left(\mathbb{E}^{m}, \mathcal{C}\right)$ over a clodum $\left(\mathcal{C}, \vee, \wedge, \star, \star^{\prime}\right)$. The difficulty here is that the set $\mathbb{T}$ of translations is a group of automorphisms when $\mathcal{C}$ is a blog and we restrict the vertical translations only by non-extreme scalars (i.e. scalars belonging to the group in $\mathcal{C}$ ), but otherwise it is only a monoid of generalized translations that are lattice dilations. Similarly the set $\mathbb{T}^{\prime}$ of dual translations is a monoid of lattice erosions. We define the kernel of $\Psi$ by

$$
\operatorname{Ker}(\Psi) \triangleq\{F: \Psi(F) \geq q\}=\left\{F: \Psi(F)(\mathbf{0}) \geq \mathcal{C}_{\mathrm{id}}\right\}
$$

where $q(x)$ is the impulse signal

$$
q(x)= \begin{cases}\mathcal{C}_{\mathrm{id}}, & x=\mathbf{0} \\ \mathcal{C}_{\mathrm{O}}, & x \neq \mathbf{0}\end{cases}
$$

$\mathcal{C}_{\text {id }}$ is the identity element of the monoid $(\mathcal{C}, \star)$, and $\mathcal{C}_{\mathrm{O}}$ is its null element. We can reconstruct the operator $\Psi$ from its kernel by adding an extra condition: we henceforth assume that $\Psi$ also commutes with adjoint operators $\lambda^{\leftarrow}$ of vertical translations $\lambda$. Thus, $\Psi$ is invariant to all combinations of horizontal translations $\mu$ and vertical translations $\lambda$ as well as to adjoint vertical translations $\lambda^{\leftarrow}$. We abbreviate this combined invariance by saying that $\Psi$ is $\mathbb{T}_{a}$-invariant. Obviously, if $\mathcal{C}$ is a blog, then all vertical translations $\lambda$ by non-extreme scalars are automorphisms whose inverses are the adjoints $\lambda^{\leftarrow}=\lambda^{-1}$; hence, in the blog case, the $\mathbb{T}$-invariance is almost identical to the $\mathbb{T}_{a}$-invariance. But in the general case $\mathbb{T}_{a}$-invariance places an extra constraint on $\Psi$.

Now observe that, for any semi-atom

$$
q_{h, v}(x)=q(x-h) \star v=\mu_{h} \lambda_{v}(q),
$$

the adjunction $\left(\lambda_{v}^{\leftarrow}, \lambda_{v}\right)$ implies that [see also (53)]

$$
q_{h, v} \leq \Psi(F) \Longleftrightarrow \lambda_{v}(q) \leq \Psi\left(\mu_{-h} F\right) \Longleftrightarrow q \leq \Psi\left(\lambda_{v}^{\leftarrow} \mu_{-h} F\right)
$$

Therefore, we can reconstruct the operator $\Psi$ from knowledge of its kernel $\operatorname{Ker}(\Psi)$ as follows:

$$
\Psi(F)(x)=\sup \left\{v \in \mathcal{C}: \lambda_{v}^{\leftarrow} \mu_{-x}(F) \in \operatorname{Ker}(\Psi)\right\}
$$

The kernel has several properties outlined next.

Proposition 24 (Maragos, 2005a).

Consider $\mathbb{T}_{a}$-operators on the $\mathcal{S}$. 
(a) If $\Psi$ is increasing and $F \in \operatorname{Ker}(\Psi)$, then $G \in \operatorname{Ker}(\Psi)$ for all $G \geq F$.

(b) If $\left\{\Psi_{i}\right\}$ is an indexed family of operators, then

$$
\operatorname{Ker}(\Psi)=\bigcup_{i} \operatorname{Ker}\left(\Psi_{i}\right) \Longrightarrow \Psi=\bigvee_{i} \Psi_{i}
$$

(c) $\operatorname{Ker}\left(\bigvee_{i} \Psi_{i}\right) \subseteq \bigcup_{i} \operatorname{Ker}\left(\Psi_{i}\right)$.

(d) $\operatorname{Ker}\left(\bigwedge_{i} \Psi_{i}\right)=\bigcap_{i} \operatorname{Ker}\left(\Psi_{i}\right)$.

(e) $\Psi_{1} \leq \Psi_{2} \Longrightarrow \operatorname{Ker}\left(\Psi_{1}\right) \subseteq \operatorname{Ker}\left(\Psi_{2}\right)$.

Example: Let $\Delta_{H}(F)=F \circledast H$ be a $\mathbb{T}$-invariant dilation, expressed as a sup- $\star$ convolution in (138), and let $\mathcal{E}_{H}$ be its adjoint erosion in (140). The kernel of this erosion is

$$
\begin{aligned}
\mathcal{K}_{H} \triangleq \operatorname{Ker}\left(\mathcal{E}_{H}\right) & =\left\{F: \mathcal{E}_{H}(F)(\mathbf{0}) \geq \mathcal{C}_{\mathrm{id}}\right\} \\
& =\left\{F: \bigwedge_{x} \lambda_{H(x)}^{\leftarrow}(F(x)) \geq \mathcal{C}_{\mathrm{id}}\right\} \\
& =\left\{F: \lambda_{H}^{\leftarrow}(F)(F(x)) \geq \mathcal{C}_{\mathrm{id}} \forall x\right\} \\
& =\left\{F: \bigvee\{v: H(x) \star v \leq F(x)\} \geq \mathcal{C}_{\mathrm{id}} \quad \forall x\right\} \\
& =\{F: H(x) \leq F(x) \forall x\} \\
& =\{F: F \geq H\}
\end{aligned}
$$

It turns out that the kernel of the above simple erosion system is the building block of the kernel of a large class of increasing operators. This leads us to the following fundamental result.

Theorem 31 (Maragos, 2005a).

Let $\Psi$ be an increasing $\mathbb{T}_{a}$-invariant operator on the $C W L$ signal space $\mathcal{S}$ and let $\left(\mathcal{E}_{H}, \Delta_{H}\right)$ be

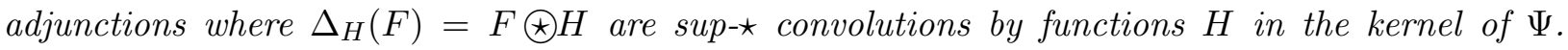
Then, $\Psi$ can be represented as the supremum of all the adjoint erosions:

$$
\Psi(F)=\bigvee_{H \in \operatorname{Ker}(\Psi)} \mathcal{E}_{H}(F)
$$

Consider now increasing operators $\Psi$ that are $\mathbb{T}_{a}^{\prime}$-invariant, i.e. invariant to all compositions of horizontal translations $\mu$ and dual vertical translations $\lambda^{\prime}$ as well as invariant to the adjoint $\lambda^{\prime \leftarrow}$ of any dual vertical translation, where $\left(\lambda^{\prime}, \lambda^{\prime \leftarrow}\right)$ is a scalar adjunction. To find kernel representations for such $\Psi$ we need to define the various kernel-related concepts in a dual way. Next we list the basic ideas and results; their derivation can be obtained by using duality on the previous results. The dual kernel of a $\mathbb{T}_{a}^{\prime}$-invariant operator $\Psi$ is defined by

$$
\operatorname{Ker}^{\prime}(\Psi) \triangleq\left\{F: \Psi(F) \leq q^{\prime}\right\}=\left\{F: \Psi(F)(\mathbf{0}) \leq \mathcal{C}_{\text {id }}^{\prime}\right\}
$$

where $\mathcal{C}_{\text {id }}^{\prime}$ is the identity element of the monoid $\left(\mathcal{C}, \star^{\prime}\right)$.

Example: Let $\mathcal{E}_{H^{\prime}}(F)=F \circledast{ }^{\prime} H^{\prime}$ be a $\mathbb{T}^{\prime}$-invariant erosion as in (143) and let $\Delta_{H^{\prime}}$ be its adjoint dilation in (144). The dual kernel of this dilation is

$$
\mathcal{K}_{H^{\prime}}^{\prime} \triangleq \operatorname{Ker}^{\prime}\left(\Delta_{H^{\prime}}\right)=\left\{F: F \leq H^{\prime}\right\}
$$

The properties of the dual kernel include the following.

Proposition 25 Consider $\mathbb{T}_{a}^{\prime}$-operators on $\mathcal{S}$.

(a) If $\Psi$ is increasing and $F \in \operatorname{Ker}^{\prime}(\Psi)$, then $G \in \operatorname{Ker}^{\prime}(\Psi)$ for all $G \leq F$.

(b) If $\left\{\Psi_{i}: i \in J\right\}$ is an indexed family of operators, then

$$
\operatorname{Ker}^{\prime}(\Psi)=\bigcap_{i} \operatorname{Ker}^{\prime}\left(\Psi_{i}\right) \Longrightarrow \Psi=\bigwedge_{i} \Psi_{i}
$$

The above results lead us to the following fundamental representation. 
Theorem 32 (Maragos, 2005a).

Let $\Psi$ be an increasing $\mathbb{T}_{a}^{\prime}$-invariant operator on the $C W L$ signal space $\mathcal{S}$ and let $\left(\mathcal{E}_{H^{\prime}}, \Delta_{H^{\prime}}\right)$ be adjunctions where $\mathcal{E}_{H^{\prime}}(F)=F \circledast{ }^{\prime} H^{\prime}$ are inf- $\star^{\prime}$ convolutions by functions $H^{\prime}$ in the dual kernel of $\Psi$. Then, $\Psi$ can be represented as the infimum of all the adjoint dilations:

$$
\Psi(F)=\bigwedge_{H^{\prime} \in \operatorname{Ker}^{\prime}(\Psi)} \Delta_{H^{\prime}}(F)
$$

\subsection{Representations for TI Openings and Closings}

In previous sections we discussed representations of increasing operators that are translationinvariant (TI). In this section we shall examine representations for TI operators that in addition to being increasing are also idempotent and extensive or anti-extensive. Namely, we shall examine TI openings and closings on the complete lattice $\mathcal{P}\left(\mathbb{E}^{m}\right)$, where $\mathbb{E}^{m}=\mathbb{R}^{m}$ or $\mathbb{Z}^{m}$. Generalizations to other complete lattices with commutative horizontal translation groups on them are possible (Ronse and Heijmans, 1991). Since a set opening (resp. closing) can be represented as supremum (resp. infimum) of its invariant sets that are included in it (resp. include it), the translation-invariance of an opening or closing is directly controlled by its invariance domain as the following result explains.

Proposition 26 A closing or an opening is translation-invariant if and only if its invariance domain is closed under translation.

Given the invariance domain $\mathcal{A}=\operatorname{Inv}(\alpha)$ of a TI opening $\alpha$, if we can find a subcollection $\mathcal{A}_{0} \subseteq$ $\mathcal{A}$ that can generate $\mathcal{A}$ via translations and (possibly infinite) set unions, we call ${ }^{26}$ it a morphological subbasis of $\mathcal{A}$. By using this concept, the following fundamental result can be proven, which states that every set opening on $\mathcal{P}\left(\mathbb{E}^{m}\right)$ can be represented as a supremum of Minkowski openings by all the structuring elements in its subbasis $\mathcal{A}_{0}$. Taking the infimum of Minkowski closings by reflected structuring elements in $\mathcal{A}_{0}$ can also represent the dual mapping of $\alpha$, which is a closing.

Theorem 33 (Matheron, 1975).

A set operator $\alpha$ on $\mathcal{P}\left(\mathbb{E}^{m}\right)$ is a translation-invariant opening if and only if there is a set collection $\mathcal{A}_{0} \subseteq \mathcal{P}\left(\mathbb{E}^{m}\right)$ such that $\alpha$ can be represented as

$$
\alpha(X)=\bigcup_{A \in \mathcal{A}_{0}} X \circ A
$$

Then, $\mathcal{A}_{0}$ is the subbasis that generates the collection $\operatorname{Inv}(\alpha)$ of $\alpha$-invariant sets. Further, the dual mapping $\beta(X)=\left(\alpha\left(X^{c}\right)\right)^{c}$ is a translation-invariant closing that can be represented as

$$
\beta(X)=\bigcap_{B \in \mathcal{A}_{0}} X \bullet B^{s}
$$

Dually, we can also work with a subbasis generating a TI closing. Specifically, a subcollection $\mathcal{B}_{0}$ of the invariance domain $\mathcal{B}=\operatorname{Inv}(\beta)$ of a $\mathrm{TI}$ closing $\beta$ is called a dual subbasis of $\mathcal{B}$ if the latter can be generated by $\mathcal{B}_{0}$ via translations and (possibly infinite) set intersections of members of $\mathcal{B}_{0}$. If $\beta$ is the dual of the above opening $\alpha$, then the two subbasis collections are related since $\mathcal{B}_{0}=\left\{A^{c}: A \in \mathcal{A}_{0}\right\}$.

Returning to the representation (280) of the TI opening via its subbasis, we observe that we can write it from the basis expansion (201) as

$$
\alpha(X)=\bigcup_{A \in \mathcal{A}_{0}} \bigcup_{a \in A} X \ominus A_{-a}=\bigcup_{a \in A \in \mathcal{A}_{0}} X \ominus A_{-a}
$$

\footnotetext{
${ }^{26}$ Matheron (1975) calls $\mathcal{A}_{0}$ a 'basis' of the TI opening; however, throughout Section 5 we have used the term 'basis' to mean the set of minimal elements of the kernel of a TI operator.
} 
To avoid redundancies in $\mathcal{A}_{0}$, we henceforth assume that $\mathcal{A}_{0}$ consists of the minimal elements of $\operatorname{Inv}(\alpha)$ that are not translations of each other. Then, the second representation in (282) is identical to the basis representation of $\alpha$, where its basis (i.e. set of minimal kernel elements) is the collection

$$
\operatorname{Bas}(\alpha)=\left\{A_{-a}: a \in A, A \in \mathcal{A}_{0}\right\}
$$

Thus, we have established the following relationship between the subbasis and basis of the invariance domain of any TI opening:

$$
\mathcal{A}_{0} \subset \operatorname{Bas}(\alpha) \subset \operatorname{Inv}(\alpha)
$$

Example 22 (Area Opening):

It can be shown that the area opening (82) of digital planar shapes, which keeps only the connected components of an input set $X \subseteq \mathbb{Z}^{2}$ with area $\geq n$, is equal to the TI opening

$$
\alpha_{n}(X)=\bigcup_{|A|=n} X \circ A
$$

where the structuring elements $A$ can be found as the connected subsets of a $n \times n$-pixel square that are not translations of each other. The collection of all these sets $A$ forms the subbasis $\mathcal{A}_{0}$. By applying $\alpha$ to all level sets $X_{v}(f)$ of an input graylevel image $f$, we can extend the above result to graylevel images and build a flat area opening:

$$
\alpha_{n}(f)=\bigvee_{|A|=n} f \circ A
$$

This max-of-openings by connected structuring elements of fixed size was introduced by Cheng and Venetsanopoulos (1992) to perform nonlinear filtering without the shape bias of a fixed structuring element. They argued heuristically against implementing $\alpha_{n}$ as a max of openings by all $A$ because this would be computationally intense due to the large number of $A$ which grows exponentially with $n$. Instead, they implemented it is as in (282), without realizing that this is essentially the basis representation.

For example, if $n=2$, there are exactly four sets $A$ in

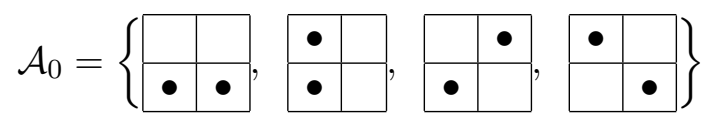

The basis $\operatorname{Bas}\left(\alpha_{n}\right)$ consists of the translations $A_{-a}, a \in A$, of all the above sets $A \in \mathcal{A}_{0}$. For the example of (287), the basis consists of 8 sets. Thus, for relatively small $n$, the area opening can be efficiently implemented as maximum of minima over the basis sets:

$$
\alpha_{n}(f)=\bigvee_{a \in A,|A|=n} f \ominus A_{-a}
$$

In another application of binary image denoising, Song and Delp (1990) used a composite morphological filter formed as union of openings by multiple connected structuring elements to remove noise more effectively than from using a single filter. In one example they used as structuring elements for the openings 8 connected sets of $n=3$ pixels each. However, this number is smaller than the total number of 3-pixel connected sets which is 20 (without counting translations of the same set). Hence, their composite filter yields a smaller output compared to the $n=3$ area opening (285); i.e. for $n=3$ the subbasis $\mathcal{A}_{0}$ contains 20 connected 3-pixel sets. Their filter can still be implemented more simply using the basis expansion (288), but it corresponds to a truncated basis. 


\section{Conclusions}

The major new contributions of this chapter are in Section 4 and in Section 5. Section 4 builds nonlinear signal spaces by introducing a new algebraic structure, called complete weighted lattices (CWLs), based on which operators that obey superpositions compatible with the basic operations of this new algebra and are translation-invariant can be represented as generalized nonlinear convolutions of the supremum or infimum type. These results provide new and strong theoretical connections between lattice-based mathematical morphology, image algebra, and minimax algebra. Section 5 unifies the morphological representation theory of operators that obey a few fundamental properties such as translation-invariance and increasingness as supremum or infimum of elementary morphological operators by extending the theory to cases where one of these properties is missing as well as to cases beyond the traditional Euclidean morphology such as the above nonlinear spaces. Section 3 provides a tutorial review of the main ideas and operators from lattice-based morphology. Similarly, Section 2 provides a synopsis of main ideas and results from linear operators on topics conceptually similar with the topics that we analyze for morphological image operators.

We have found many interesting analogies between linear operators and the new view of morphological operators on complete weighted lattices. The most interesting and fundamentally important is the striking similarity between the algebraic structure of a linear space and that of the (nonlinear) complete weighted lattice space which we introduced in this chapter. Another striking similarity is between the linear convolution representation of a linear shift-invariant operator and the supremal or infimal convolution of weighted lattice operators that obey a weighted supremum or infimum superposition and some kind of generalized translation-invariance. In the finite-dimensional case, this becomes another impressive similarity between the linear algebra matrix representation of a linear operator over traditional vector spaces and the max-plus (or more general max- $\star$ ) matrix representation of a dilation or erosion operator over finite-dimensional complete weighted lattices. Of possible interest in the future is also to explore possible analogies between the pair formed by a linear operator and its adjoint with the lattice adjunction pair of an erosion and its adjoint dilation. Compared with linear operators, an area that is still not well developed in morphological and lattice operators is the class of projections. For instance, missing is a lattice-based 'projection theorem', whereas the corresponding projection theorem in Hilbert spaces has proven to be extremely useful as an interdisciplinary tool for approximate modeling. In this chapter we have only observed some conceptual analogies between linear projections and morphological projections and we have analyzed several lattice-theoretic aspects of the latter. Another area which is well developed in linear operators is their spectral decomposition. However, with the exception of the slope transform, it seems almost absent from morphological operators. Only some work has been done in eigenvalue-eigenvector analysis in max-plus algebra, but the developments are still far from the vast knowledge that has accumulated in linear algebra. Finally, missing seems to be for morphological operators a study of their continuity based on some norm, at some level close to what is done for linear operators on normed spaces. The only results available deal with their order-based (semi-)continuity and its relationship with topological (semi-)continuity.

In representation theory, we have covered a broad spectrum of ideas and results from morphological operators and their lattice generalizations, focusing on their theoretic representations of two types: (1) As supremal or infimal convolutions, in the case where we have operators that obey a supremum or infimum superposition and translation-invariance, either Euclidean or some invariance w.r.t. a (semi-)group of generalized 'translations' . (2) As parallel combinations (supremum or infimum superpositions) of simpler morphological operations (elementary erosions or dilations), in the case where we have operators that are increasing and/or translation-invariant (TI). The representation theory of increasing and TI operators encompasses a very broad variety of nonlinear and linear systems. In this chapter we have provided examples from several categories of systems to which this theory applies. This class of operators is sup-generated by elementary erosions with basis functions or inf-generated by elementary dilations with the dual basis functions. Namely, in the lattice of increasing and TI operators, the erosions by basis elements are atoms that can synthe- 
size more complex operators. Exact representation requires all the atoms; thus the basis expansion is irreducible. Of great practical and theoretical significance are the approximate representations using a truncated morphological basis.

\section{Acknowledgments:}

This chapter was written while the author was visiting the Laboratory of Information and Decision Systems at the Massachusetts Institute of Technology whose hospitality and facilities are gratefully acknowledged. The work was partially supported by the basic research grant COGNIMUSE which is implemented under the "ARISTEIA Action of the Operational Program Education and Lifelong Learning and is co-funded by the European Social Fund and Greek National Resources.

\section{References}

Alvarez, L., Baumela, L., Henriquez, P., and Marquez-Neila, P. (2010). Morphological snakes. In Proc. IEEE Conf. Computer Vision \& Pattern Recognition.

Alvarez, L., Guichard, F., Lions, P., and Morel, J.-M. (1993). Axioms and fundamental equations of image processing. Archiv. Rat. Mech., 123(2):199-257.

Banon, G. J. and Barrera, J. (1991). Minimal representations for translation-invariant set mappings by mathematical morphology. SIAM J. Appl. Math., 51:1782-1798.

Banon, G. J. and Barrera, J. (1993). Decomposition of mappings between complete lattices by mathematical morphology. Signal Processing, 30:299-327.

Barrera, J. and Banon, G. J. (1992). Expressiveness of the morphological language. In Image Algebra and Morphological Image Processing III, volume 1769 of Proc. SPIE, pages 264-275.

Barrera, J., Banon, G. J., and Lotufo, R. (1994). A mathematical morphology toolbox for the khoros system. In Image Algebra and Morphological Image Processing V, volume 2300 of Proc. SPIE, page 241252 .

Barrera, J. and Dougherty, E. R. (1998). Representation of gray-scale windowed operators. In Heijmans, H. and Roerdink, J., editors, Mathematical Morphology and Its Applications to Image and Signal Processing, pages 19-26. Kluwer Acad. Publ.

Barrera, J., Dougherty, E. R., and Tomita, N. S. (1997). Automatic programming of binary morphological machines by design of statistically optimal operators in the context of computational learning theory. J. Electron. Imaging, 6(1):54-67.

Barrera, J. and Salas, G. P. (1996). Set operations on closed intervals and their applications to the automatic programming of morphological machines. J. Electron. Imaging, (5):335-352.

Bellman, R. and Karush, W. (1963). On the maximum transform. J. Math. Anal. Appl., 6:67-74.

Birkhoff, G. (1967). Lattice Theory. Amer. Math. Soc., Providence, Rhode Island.

Bloch, I. and Maitre, H. (1995). Fuzzy Mathematical Morphologies: a Comparative Study. Pattern Recognition, 9(28):1341-1387.

Blyth, T. S. and Janowitz, M. F. (1972). Residuation Theory. Pergamon Press, Oxford.

Borgefors, G. (1986). Distance transformations in digital images. Computer Vision, Graphics, and Image Processing, 34:344-371. 
Bouaynaya, N., Charif-Chefchaouni, M., and Schonfeld, D. (2008). Theoretical foundations of spatially-variant mathematical morphology part i: Binary images. IEEE Trans. Pattern Analysis and Machine Intelligence, 30(5):823-836.

Bouaynaya, N. and Schonfeld, D. (2008). Theoretical foundations of spatially-variant mathematical morphology part ii: Gray-level images. IEEE Trans. Pattern Analysis and Machine Intelligence, 30(5):837-850.

Braga-Neto, U. (1996). Alternating sequential filters by adaptive-neighborhood structuring functions. In Maragos, P., Schafer, R., and Butt, M., editors, Mathematical Morphology and Its Applications to Image and Signal Processing, pages 139-146. Kluwer Acad. Publ.

Breen, E. J. and Jones, R. (1996). Attribute openings, thinnings, and granulometries. Computer Vision and Image Understanding, 64(3):377389.

Brockett, R. W. and Maragos, P. (1994). Evolution equations for continuous-scale morphological filtering. IEEE Trans. Signal Processing, 42(12):3377-3386.

Catte, F., Dibos, F., and Koepfler, G. (1995). A Morphological Scheme for Mean Curvature Motion and Applications to Anisotropic Diffusion and Motion of Level Sets. SIAM J. Num. Analysis, 32:1895-1909.

Chatzis, V. and Pitas, I. (2000). A Generalized Fuzzy Mathematical Morphology and Its Application in Robust 2-D and 3-D Object Representation. IEEE Trans. Image Processing, 9:1798-1810.

Cheng, F. and Venetsanopoulos, A. N. (1992). An adaptive morphological filter for image processing. IEEE Trans. Image Process., 1(4):533-539.

Cohen, G., Moller, P., Quadrat, J., and Viot, M. (1989). Algebraic Tools for the Performance Evaluation of Discrete Event Systems. Proceedings of the IEEE, 77:39-58.

Crimmins, T. R. and Brown, W. R. (1985). Image Algebra and Automatic Shape Recognition. IEEE Trans. Aerosp. and Electron. Syst., pages 60-69.

Cuninghame-Green, R. (1979). Minimax Algebra. Springer-Verlag.

Cuninghame-Green, R. (1994). Minimax Algebra and Applications. In Hawkes, P. W. and Kazan, B., editors, Advances in Imaging and Electron Physics, volume 90, pages 1-121. Acad. Press.

Davidson, J. L. (1993). Classification of lattice transformations in image processing. Computer Vision, Graphics, and Image Processing, 57(3):283306.

Davidson, J. L. and Hummer, F. (1993). Morphology neural networks: An introduction with applications. Circuits, Systems, and Signal Processing, 12(2):177-210.

Debayle, J. and Pinoli, J.-C. (2006). General adaptive neighborhood image processing: Part i: Introduction and theoretical aspects. J. Math. Imaging Vis., 25(2):245-266.

Deng, T. Q. and Heijmans, H. (2002). Grey-Scale Morphology Based on Fuzzy Logic. J. Math. Imaging and Vision, 16:155-171.

Dorst, L. and van den Boomgaard, R. (1994). Morphological Signal Processing and the Slope Transform. Signal Processing, 38:79-98.

Dougherty, E. R. and Astola, J. (1994). An Introduction to Nonlinear Image Processing, volume TT16. SPIE Press, Bellingham.

Dougherty, E. R. and Kraus, E. J. (1991). Shape Analysis and Reduction of the Morphological Basis For Digital Moving-Average Filters. SIAM J. Appl. Math., 51(6):1764-1781. 
Felzenszwalb, P. F. and Huttenlocher, D. P. (2004a). Distance transforms of sampled functions. Technical Report TR2004-1963, Cornell University.

Felzenszwalb, P. F. and Huttenlocher, D. P. (2004b). Efficient Belief Propagation for Early Vision. In Proc. IEEE Conf. Computer Vision \& Pattern Recognition.

Gierz, G., Hoffman, K. H., Keimel, K., Lawson, J. D., Mislove, M., and Scott, D. S. (1980). A Compendium of Continuous Lattices. Springer-Verlag.

Gilbert, E. N. (1954). Lattice-theoretic properties of frontal switching functions. J. Mathematical Physics, 33:57-67.

Goetcherian, V. (1980). From Binary To Greytone Image Processing Using Fuzzy Logic Concepts. Pattern Recognition, 12:7-15.

Goutsias, J. (1992). Morphological Analysis of Discrete Random Shapes. J. Math. Imaging and Vision, 2:193-215.

Goutsias, J., Heijmans, H., and Sivakumar, K. (1995). Morphological operators for image sequences. Computer Vision and Image Understanding, 62(3):326-346.

Guichard, F., Maragos, P., and Morel, J.-M. (2005). Partial differential equations for morphological operators. In Bilodeau, M., Meyer, F., and Schmitt, M., editors, Space, Structure, and Randomness, Lec. Notes in Statistics, pages 369-390. Springer. Contributions in Honor of Georges Matheron in the Fields of Geostatistics, Random Sets, and Mathematical Morphology.

Guichard, F. and Morel, J.-M. (2001). Image Analysis and P.D.E.s. Lecture Notes, ENS-Cachan, Paris.

Hadwiger, H. (1957). Vorlesungen über Inhalt, Oberfläche, und Isoperimetrie. Springer Verlag, Berlin.

Halmos, P. R. (1963). Lectures on Boolean Algebras. D. Van Nostrand Co., Princeton.

Haralick, R. M. and Shapiro, L. G. (1992). Computer and Robot Vision, volume I. Addison-Wesley.

Haralick, R. M., Sternberg, S. R., and Zhuang, X. (1987). Image analysis using mathematical morphology. IEEE Trans. Pattern Analysis and Machine Intelligence, 9(4):532-550.

Harber, R. G., Bass, S. C., and Neudeck, G. W. (1985). VLSI Implementation of a Fast Rank Order Filtering Algorithm. In Proc. IEEE ICASSP, Tampa, FL.

Heijmans, H. (1987). Mathematical Morphology: An Algebraic Approach. CWI Newsletter, (14):727.

Heijmans, H. (1994). Morphological Image Operators. Acad. Press, Boston.

Heijmans, H. and Maragos, P. (1997). Lattice calculus of the morphological slope transform. Signal Processing, 59:17-42.

Heijmans, H., Nacken, P., Toet, A., and Vincent, L. (1992). Graph morphology. J. Vis. Comun. Image Represent., 3(1):24-38.

Heijmans, H. and Ronse, C. (1990). The algebraic basis of mathematical morphology. part i: Dilations and erosions. Computer Vision, Graphics, and Image Processing, 50:245-295.

Hereford, J. M. and Rhodes, W. T. (1988). Nonlinear optical image filtering by time-sequential threshold decomposition. Optical Enginr. 
Kaburlasos, V. and Petridis, V. (2000). Fuzzy Lattice Neurocomputing (FLN) Models. Neural Networks, 13:1145-1169.

Keshet, R. (2000). Mathematical morphology on complete semilattices and its applications to image processing. Fundamentae Informatica, 41:33-56.

Kiselman, C. O. (2003). Digital Geometry and Mathematical Morphology. Lecture Notes, Uppsala University.

Klir, G. J. and Yuan, B. (1995). Fuzzy Sets and Fuzzy Logic: Theory and Applications. PrenticeHall.

Ko, S.-J., Morales, A., and Lee, K.-H. (1995). Block basis matrix implementation of the morphological open-closing and close-opening. IEEE Signal Processing Letters, 2(1):7-9.

Kohavi, Z. (1978). Switching and Finite Automation Theory. McGraw-Hill.

Koshravi, M. and Schafer, R. W. (1994). Implementation of linear digital filters based on morphological representation theory. IEEE Trans. Signal Processing, 42(9):2264-2275.

Lang, S. (2005). Algebra. Springer, rev. 3rd edition.

Lerallut, R., Decenciere, E., and Meyer, F. (2005). Image Filtering Using Morphological Amoebas. In Ronse, C., Najman, L., and Decenciere, E., editors, Mathematical Morphology: 40 Years On, pages 13-22. Springer-Verlag.

Loce, R. P. and Dougherty, E. R. (1992a). Facilitation of optimal binary morphological filter design via structuring element libraries and design constraints. Optical Engineering, 31:1008-1025.

Loce, R. P. and Dougherty, E. R. (1992b). Optimal Morphological Restoration: The Morphological Filter Mean-Absolute-Error Theorem. J. Visual Communication and Image Representation, $3(4): 414-432$.

Loce, R. P. and Dougherty, E. R. (1995). Mean-absolute-error representation and optimization of computational-morphological filters. Graphical Models and Image Processing, 57(1):27-37.

Lucet, Y. (2010). What shape is your conjugate? a survey of computational convex analysis and its applications. SIAM Review, 52(3):505-542.

Maragos, P. (1985). A Unified Theory of Translation-Invariant Systems With Applications to Morphological Analysis and Coding of Images. PhD thesis, Georgia Inst. Technology, Atlanta, USA.

Maragos, P. (1989a). A Representation Theory for Morphological Image and Signal Processing. IEEE Trans. Pattern Analysis and Machine Intelligence, 11:586-599.

Maragos, P. (1989b). Pattern Spectrum and Multiscale Shape Representation. IEEE Trans. Pattern Analysis and Machine Intelligence, 11:701-716.

Maragos, P. (1990). Affine morphology and affine signal models. In Gader, P. D., editor, Image Algebra and Morphological Image Processing, volume 1350 of Proc. SPIE, pages 31-43.

Maragos, P. (1994). Morphological systems: Slope transforms and max-min difference and differential equations. Signal Processing, 38:57-77.

Maragos, P. (1995). Slope Transforms: Theory and Application to Nonlinear Signal Processing. IEEE Trans. Signal Processing, 43(4):864-877. 
Maragos, P. (1996). Differential morphology and image processing. IEEE Trans. Image Processing, 78:922-937.

Maragos, P. (1998). Morphological signal and image processing. In Madisetti, V. and Williams, D., editors, Digital Signal Processing Handbook. CRC Press.

Maragos, P. (2001). Differential morphology. In Mitra, S. and Sicuranza, G., editors, Nonlinear Image Processing, pages 289-329. Acad. Press.

Maragos, P. (2005a). Lattice image processing: A unification of morphological and fuzzy algebraic systems. J. Math. Imaging and Vision, 22:333-353.

Maragos, P. (2005b). Morphological filtering for image enhancement and feature detection. In Bovik, A., editor, Image and Video Processing Handbook, pages 135-156. Elsevier Acad. Press, 2 edition.

Maragos, P. (2005c). Partial differential equations for morphological scale-spaces and eikonal applications. In Bovik, A., editor, Image and Video Processing Handbook, pages 587-612. Elsevier Acad. Press, 2 edition.

Maragos, P. and Schafer, R. W. (1987a). Morphological Filters - Part I: Their Set-Theoretic Analysis and Relations to Linear Shift-Invariant Filters. IEEE Trans. Acoustics, Speech, and Signal Processing, 35:1153-1169.

Maragos, P. and Schafer, R. W. (1987b). Morphological Filters - Part II: Their Relations to Median, Order-Statistic, and Stack Filters. IEEE Trans. Acoustics, Speech, and Signal Processing, 35:1170-1184. "Corrections," IEEE Trans. ASSP, vol.37, no.4, p.597, Apr. 1989.

Maragos, P. and Schafer, R. W. (1990). Morphological systems for multidimensional signal processing. Proceedings of the IEEE, 78:690-710.

Maragos, P., Stamou, G., and Tzafestas, S. G. (2000). A lattice control model of fuzzy dynamical systems in state-space. In Goutsias, J., Vincent, L., and Bloomberg, D., editors, Mathematical Morphology and Its Application to Image and Signal Processing. Kluwer Acad. Publ., Boston.

Maragos, P. and Tzafestas, S. G. (1999). Max-Min Control Systems with Applications to Discrete Event Dynamical Systems. In Tzafestas, S., editor, Advances in Manufacturing: Decision, Control and Information Technology, pages 217-230. Springer-Verlag.

Maragos, P., Tzouvaras, V., and Stamou, G. (2001). Synthesis and Applications of Lattice Image Operators Based On Fuzzy Norms. In Proc. Int'l Conf. on Image Processing, Thessaloniki, Greece.

Maragos, P., Tzouvaras, V., and Stamou, G. (2003). Lattice Fuzzy Image Operators and Generalized Image Gradients. In Proc. Int'l Fuzzy Systems Assoc. World Congress (IFSA-2003), number 2715 in LNCS, pages 412-419, Turkey. Springer-Verlag.

Maragos, P. and Vachier, C. (2009). Overview of adaptive morphology: Trends and perspectives. In Proc. Int'l Conf. Image Processing (ICIP-2009), Cairo, Egypt.

Maragos, P. and Ziff, R. D. (1990). Threshold superposition in morphological image analysis systems. IEEE Trans. Pattern Analysis and Machine Intelligence, 12:498-504.

Marr, D. (1982). Vision. W.H. Freeman.

Matheron, G. (1975). Random Sets and Integral Geometry. Wiley, New York. 
Meyer, F. (1978). Contrast Feature Extraction. In Special Issues of Practical Metallography. ederer Verlag GmbH, Stuttgart. Proc. 2nd European Symp. on Quant. Anal. of Microstruct. in Materials Science, Biology and Medicine, France, Oct. 1977.

Meyer, F. and Maragos, P. (2000). Nonlinear Scale-Space Representation with Morphological Levelings. J. Visual Communication and Image Representation, 11:245-265.

Minkowski, H. (1903). Volumen und Oberflache. Math. Annalen, 57:447-495.

Morales, A. and Acharya, R. (1993). Statistical analysis of morphological openings. IEEE Trans. Signal Processing, 41(10):3052-3056.

Muroga, S. (1971). Threshold Logic and Its Applications. Wiley.

Nachtegael, M. and Kerre, E. E. (2001). Connections Between Binary, Gray-scale and Fuzzy Mathematical Morphologies. Fyzzy Sets and Systems, 124:73-85.

Nakagawa, Y. and Rosenfeld, A. (1978). A Note on the Use of Local Min and Max Operations in Digital Picture Processing. IEEE Trans. Syst., Man, and Cybern., 8:632-635.

Naylor, A. W. and Sell, G. R. (1982). Linear Operator Theory in Engineering and Science. SpringerVerlag.

Ochoa, E., Allebach, J. P., and Sweeney, D. W. (1987). Optical median filtering by threshold decomposition. Appl. Opt., 26:252-260.

O'Neil, K. S. and Rhodes, W. T. (1986). Morphological transformations by hybrid optical-electronic methods. In Casasent, D., editor, Hybrid Image Processing, volume 638 of Proc. SPIE, pages $41-44$.

Ouzounis, G. and Wilkinson, M. (2007). Mask-based second-generation connectivity and attribute filters. IEEE Trans. Pattern Anal. Mach. Intellig., 29:990-1004.

Pessoa, L. F. and Maragos, P. (1998). MRL-Filters: A General Class of Nonlinear Systems and Their Optimal Design for Image Processing. IEEE Trans. Image Processing, 7(7):966-978.

Pessoa, L. F. and Maragos, P. (2000). Neural networks with hybrid morphological/rank/linear nodes: a unifying framework with applications to handwritten character recognition. Pattern Recognition, 33:945-960.

Peteanu, V. (1967). An algebra on the optimal path in networks. Mathematica, 9:335342.

Preston, K., Duff, M., Levialdi, S., Norgren, P. E., and Toriwaki, J.-I. (1979). Basics of cellular logic with some applications in medical image processing. procieee, 67:826-856.

Ritter, G. X. and Gader, P. D. (1987). Image Algebra Techniques for Parallel image Processing. J. Paral. Distr. Comput., 4:7-44.

Ritter, G. X., Sussner, P., and de Leon, J. L. D. (1998). Morphological associative memories. IEEE Trans. Neural Networks, 9(2):281-293.

Ritter, G. X. and Urcid, G. (2003). Lattice algebra approach to single-neuron computation. IEEE Trans. Neural Networks, 14(2):282-295.

Ritter, G. X. and Wilson, J. N. (1987). Image algebra in a nutshell. In Proc. Int'l Conf. on Computer Vision, pages 641-645, London.

Ritter, G. X. and Wilson, J. N. (2001). Handbook of Computer Vision Algorithms in Image Algebra. CRC Press, 2 edition. 
Rockafellar, R. T. (1970). Convex Analysis. Princeton Univ. Press, Princeton.

Roerdink, J. (1993). Mathematical morphology with noncommutative symmetry groups. In Dougherty, E., editor, Mathematical Morphology in Image Processing, pages 205-254. Marcel Dekker.

Roerdink, J. (2000). Group morphology. Pattern Recognition, 33:877-895.

Roerdink, J. (2009). Adaptivity and group invariance in mathematical morphology. In Proc. Int'l Conf. Image Processing (ICIP-2009).

Roerdink, J. and Heijmans, H. (1988). Mathematical morphology for structures without translation symmetry. Signal Processing, 15:271-277.

Ronse, C. and Heijmans, H. (1991). The Algebraic Basis of Mathematical Morphology. Part II: Openings and Closings. Computer Vision, Graphics, and Image Processing: Image Understanding, 54:74-97.

Royden, H. L. (1968). Real Analysis. Macmillan Publ., NY, 2nd edition.

Salembier, P. and Serra, J. (1995). Flat Zones Filtering, Conencted Operators, and Filters by Reconstruction. IEEE Trans. Image Processing, 4:1153-1160.

Schonfeld, D. and Goutsias, J. (1991). Optimal Morphological Pattern Restoration from Noisy Binary Images. IEEE Trans. Pattern Analysis and Machine Intelligence, 13:14-29.

Serra, J. (1982). Image Analysis and Mathematical Morphology. Acad. Press.

Serra, J., editor (1988). Image Analysis and Mathematical Morphology, volume 2: Theoretical Advances. Acad. Press.

Serra, J. (2000). Connections for Sets and Functions. Fundamentae Informatica, 41:147-186.

Serra, J. and Salembier, P. (1993). Connected Operators and Pyramids. In Image Algebra and Mathematical Morphology, volume 2030 of Proc. SPIE, pages 65-76.

Serra, J. and Vincent, L. (1992). An overview of morphological filtering. Circuits, Systems, and Signal Processing, 11(1):47-108.

Sinha, D. and Dougherty, E. R. (1992). Fuzzy mathematical morphology. J. Visual Communication and Image Representation, 3(3):286-302.

Song, J. and Delp, E. J. (1990). The analysis of morphological filters with multiple structuring elements. Computer Vision, Graphics, and Image Processing, 50:308-328.

Sternberg, S. R. (1980). Language and architecture for parallel image processing. In Gelsema, E. and Kanal, L., editors, Pattern Recognition in Practice. North Holland Publ.

Sternberg, S. R. (1986). Grayscale morphology. Computer Vision, Graphics, and Image Processing, 35:333-355.

Svalbe, I. D. (1991). The geometry of basis sets for morphologic closing. IEEE Trans. Pattern Analysis and Machine Intelligence, 13(12):1214-1224.

van den Boomgaard, R. and Smeulders, A. (1994). The morphological structure of images: The differential equations of morphological scale-space. IEEE Trans. Pattern Analysis and Machine Intelligence, 16:1101-1113.

Vincent, L. (1989). Graphs and mathematical morphology. Signal Processing, 16(4):365-388. 
Vincent, L. (1992). Morphological Area Openings and Closings for Grayscale Images. In Proc NATO Workshop on "Shape in Picture, pages 197-208, Driebengen, The Netherlands. Springer-Verlag.

Wendt, P. D., Coyle, E. J., and Gallagher, N. C. (1986). Stack Filters. IEEE Trans. Acoust., Speech, Signal Process., 34:898-911.

Yang, P.-F. and Maragos, P. (1995). Min-Max Classifiers: Learnability, Design And Application. Pattern Recognition, 28(6):879-899.

Zadeh, L. A. (1965). Fuzzy Sets. Information and Control, 8:338-353. 